\title{
Mechanische Spektroskopie an metallischen Gläsern in reduzierter Dimensionalität
}

\author{
Dissertation
}

\author{
zur Erlangung des Doktorgrades \\ der Mathematisch-Naturwissenschaftlichen Fakultäten \\ der Georg-August-Universität zu Göttingen
}

vorgelegt von

Dennis Bedorf

aus Dortmund

Göttingen, 2009 
D7

Referent: Prof. Dr. Konrad Samwer Korreferent: Prof. Dr. Hans-Ulrich Krebs

Tag der mündlichen Prüfung: 28.10.2009 


\section{Inhaltsverzeichnis}

1 Einleitung und Motivation 1

1.1 Ziel dieser Arbeit . . . . . . . . . . . . . . . . . . . . 1

1.2 Amorphe Festkörper, Gläser und Schmelzen . . . . . . . . . 3

1.2.1 Gläser, Glasübergang . . . . . . . . . . . . . . . 3

1.2 .2 Metallische Gläser . . . . . . . . . . . . . 4

1.3 Dynamik in Gläsern . . . . . . . . . . . . . 5

1.3.1 Beiträge zur Dynamik . . . . . . . . . . 5

1.3.2 Temperaturabhängigkeit der Dynamik, ,Merging“ . . . 7

1.3.3 Alterungen ..................... 11

1.4 Heterogenitäten und Längenskalen in Gläsern . . . . . . . . . 12

2 Mechanische Spektroskopie $\quad 15$

2.1 Allgemeine Begriffe der mechanischen Spektroskopie . . . . . . 15

2.1.1 Dynamisch-mechanische Messungen . . . . . . . . 16

2.2 Relaxationsmessungen . . . . . . . . . . . . . . . 17

2.2.1 Debye-Relaxation . . . . . . . . . . . . . . . 18

2.2.2 Allgemeine Relaxationsspektren . . . . . . . . . . . 19

2.3 Wichtige Messmethoden . . . . . . . . . . . . . 21

2.4 Der Doppelpaddel-Oszillator . . . . . . . . . . . . . . 24

2.4.1 Eigenmoden . . . . . . . . . . . . . . . 24

2.4.2 Bestimmung der Dämpfung . . . . . . . . . . . 27

2.4.3 Einfluss des Films auf das DPO . . . . . . . . . . . 28

2.4.4 Reproduzierbarkeit und Messempfindlichkeit . . . . . 31

3 Experimentelles 33

3.1 Der DPO-Messaufbau . . . . . . . . . . . . . 33

3.1.1 Temperatursteuerung . . . . . . . . . . . 36

3.1.2 Datenaufnahme . . . . . . . . . . . . . . 42

3.2 Herstellung, Vorbereitung der DPO . . . . . . . . . . 46

3.2.1 Herstellung . . . . . . . . . . . . . . . . 46

3.2 .2 Vorbereitung . . . . . . . . . . . . 47 
3.3 Herstellung dünner PdCuSi-Schichten . . . . . . . . . . . . . . . 49

3.3.1 Die Balzers UHV-Anlage . . . . . . . . . . . . . . . 49

3.3.2 Der Aufdampfprozess . . . . . . . . . . . . . . 50

3.4 Weitere Analysen dünner Schichten . . . . . . . . . . . 51

3.4.1 Kleinwinkel-Röntgenstreuung . . . . . . . . . . . . 51

3.4.2 Weitwinkel-Röntgenstreuung . . . . . . . . . . . 52

3.4.3 Rasterelektronenmikroskopie mit EDX . . . . . . . . 52

3.4.4 Rastersondenmikroskopie . . . . . . . . . . . . 53

4 Ergebnisse $\quad 55$

4.1 Charakterisierung der Schichten . . . . . . . . . . 55

4.1.1 Zusammensetzung der Schichten, EDX . . . . . . . 56

4.1.2 Strukturelle Analyse mit XRD . . . . . . . . . . . . 58

4.1.3 Oberflächentopographien . . . . . . . . . . . . 60

4.2 Mechanische Messungen . . . . . . . . . . . . . . . . 64

4.2.1 Unbeschichtete Doppelpaddel-Oszillatoren . . . . . . . 64

4.2.2 Mechanische Analyse einer dicken PdCuSi-Schicht . . . 67

4.2.3 Mechanische Messungen unter Raumtemperatur . . . . 72

4.2.4 Mechanische Charakterisierung dünner amorpher Filme 75

4.2.5 Einfluss der thermischen Vorbehandlung . . . . . . . . 85

4.2.6 Mechanische Eigenschaften nahe Raumtemperatur . . . 85

5 Diskussion $\quad 89$

5.1 Qualität der Schichten, Morphologie . . . . . . . . . . 89

5.1.1 Stöchiometrie . . . . . . . . . . . . . . 89

5.1.2 Rauigkeiten und Morphologie . . . . . . . . . . . . 90

5.2 Genauigkeit und Verlustprozesse . . . . . . . . . . . . . . . . 92

5.3 Diskussion neuer Resultate . . . . . . . . . . . . . . . . . 95

5.3.1 Vergleich mit PdCuSi-Bändern . . . . . . . . . . 95

5.3.2 Vergleich mit dem starken Glas $\mathrm{ZrAlCu}$. . . . . . . . 99

5.3.3 Das Potentielle-Energie-Landschafts-Modell . . . . . . 100

5.4 Relaxationen in Gläsern . . . . . . . . . . . . . . . . . . . . 102

5.5 Einfluss der Probengröße . . . . . . . . . . . . . . . . 106

5.5.1 Dünne Filme auf rauen und glatten Substraten . . . . 106

5.5.2 Analyse der dünnen Filme auf glatten Substraten . . . 107

5.5.3 Grenzfall: $10 \mathrm{~nm}$ Schichtdicke . . . . . . . . . . . . 110

5.5.4 Glastemperatur in Abhängigkeit der Schichtdicke . . . 111

5.5.5 Modellbildung . . . . . . . . . . . . . . . . 114

5.5.6 Vergleich zu anderen Längenskaleneffekten . . . . . . . 120

$\begin{array}{lll}6 & \text { Zusammenfassung } & 123\end{array}$ 
$\begin{array}{lr}\text { A Anhang } & 127\end{array}$

A.1 Weitere Röntgenmessungen . . . . . . . . . . . . . . 127

A.2 Weitere mechanische Messungen . . . . . . . . . . . 128

Literaturverzeichnis 


\section{Abbildungsverzeichnis}

1.1 Schematische Darstellung eines Glasübergangs . . . . . . . . . 4

1.2 Relaxationsspektrum eines organischen Glasbildners . . . . . . 6

1.3 Viskositätsänderung von Glasbildnern oberhalb der Glastemperatur. . . . . . . . . . . . . . 7

1.4 Entkopplung von Zeitskalen der Vibrationen von strukturellen Zeitkonstanten . . . . . . . . . . . . 8

1.5 Relaxationszeiten von o-Terphenyl . . . . . . . . . . . . . 9

1.6 Aktivierungsplot für $\mathrm{PdCuSi}$. . . . . . . . . . . . . . . . . . 10

1.7 Partielle radiale Strukturfunktion . . . . . . . . . . . . . . . 12

1.8 Darstellung kolloider Partikel . . . . . . . . . . . . . . . . . 14

2.1 Ersatzschaltbild eines standard linear solid - Modells . . . . . 19

2.2 Darstellung einer Debye-Relaxation . . . . . . . . . . . . . 20

2.3 Schematische Darstellung einiger DPO-Moden . . . . . . . . . 25

2.4 Frequenzspektrum mit bekannten Moden . . . . . . . . . . . 26

2.5 Amplitudenverlauf beim "Ring-down" . . . . . . . . . . 28

3.1 Der UHV Einsatz „DPO Evolution“. . . . . . . . . . . . . 34

3.2 Detailansicht des Messeinsatzes mit eingebautem Paddel. . . . 37

3.3 Verlauf von Klemmblocktemperatur und Paddelkopftemperatur 38

3.4 Messung der Resonanzfrequenz bei $40{ }^{\circ} \mathrm{C}$. . . . . . . . . . . . 39

3.5 Temperaturverlauf beim konstanten Heizen auf $350{ }^{\circ} \mathrm{C}$. . . . 40

3.6 Vereinfachte Darstellung der verwendeten Schaltung. . . . . . 44

3.7 Aufsicht auf einen bedampften Doppelpaddel-Oszillator . . . . 46

3.8 REM-Aufnahme eines lasergeschnittenen Paddels . . . . . . . 47

3.9 REM-Aufnahme der Kantendetails nach dem Laserschneiden . 48

4.1 EDX-Spektrum einer $50 \mathrm{~nm} \mathrm{PdCuSi-Schicht} \mathrm{.} \mathrm{.} \mathrm{.} \mathrm{.} \mathrm{.} \mathrm{.} \mathrm{.} \mathrm{.} \mathrm{.} 57$

4.2 EDX-Spektrum einer $50 \mathrm{~nm}$ PdCuSi-Schicht, vergrößert . . . . 57

4.3 Weitwinkel-Röntgenspektrum der Probe DPO_DB_08 . . . . 58

4.4 STM-Bild einer $50 \mathrm{~nm}$ Tantal Schicht auf Silizium . . . . . . . 61 
4.5 REM-Aufnahme einer $50 \mathrm{~nm}$ Tantal Schicht auf Silizium . . . 62

4.6 STM-Bild eines kristallisierten PdCuSi-Films mit $50 \mathrm{~nm}$ Dicke. 62

4.7 AFM-Bild einer $10 \mathrm{~nm}$ PdCuSi-Schicht . . . . . . . . . . 63

4.8 Leermessung von DPO_DB_05 mit Kühlen und Heizen . . . 64

4.9 Anpassung des Dämpfungsverlaufs von DPO_DB_05 . . . . . 65

4.10 Zusammenstellung verschiedener Dämpfungsmessungen . . . . 66

4.11 Temperaturverlauf der AS2-Mode für DPO_DB_05 . . . . . 67

4.12 Einfluss von Kontaminationen vor dem Ausheizen . . . . . . . 68

4.13 Verlauf der Dämpfung des DPOs mit einer $200 \mathrm{~nm} \mathrm{PdCuSi-}$ Schicht im Vergleich zu dem leeren Paddel . . . . . . . . . . . 69

4.14 Dämpfungssignal der $200 \mathrm{~nm}$ PdCuSi-Schicht . . . . . . . . . 69

4.15 Verlauf der Resonanzfrequenz von DPO_DB_05 . . . . . 70

4.16 Frequenzverschiebung durch den $200 \mathrm{~nm}$ PdCuSi-Film . . . . 71

4.17 Berechneter komplexer Schermodul der $200 \mathrm{~nm}$ PdCuSi-Schicht 72

4.18 Messung der Dämpfung eines DPOs vergleichend mit $200 \mathrm{~nm}$ amorpher PdCuSi-Schicht und unbeschichtet . . . . . . . 73

4.19 Dämpfungsdifferenz durch die $200 \mathrm{~nm}$ PdCuSi-Schicht beim Abkühlen ...................... . 73

4.20 Messung der Dämpfung beim Gegenheizen . . . . . . . . . . . 74

4.21 Vergleich der Frequenzverschiebung . . . . . . . . . . . . 75

4.22 Messung des PdCuSi-Films von $50 \mathrm{~nm}$ auf rauem Substrat . . 76

4.23 Dämpfungsverlauf des Paddels DPO_DB_07 . . . . . . . . 77

4.24 Dämpfung des $50 \mathrm{~nm}$ PdCuSi-Filmes auf dem glatten Substrat 78

4.25 Frequenzverschiebung durch den 50 nm PdCuSi-Film . . . . . 79

4.26 Messung der Dämpfung mit $25 \mathrm{~nm} \mathrm{PdCuSi}$. . . . . . . . . . . 80

4.27 Übersicht der Verlustmessungen . . . . . . . . . . . . . . . . . . 81

4.28 Messung der Dämpfung mit $10 \mathrm{~nm} \mathrm{PdCuSi}$. . . . . . . . . . . 82

4.29 Verlust durch die $10 \mathrm{~nm} \mathrm{PdCuSi-Schicht} \mathrm{.} \mathrm{.} \mathrm{.} \mathrm{.} \mathrm{.} \mathrm{.} \mathrm{.} \mathrm{.} \mathrm{.} \mathrm{.} \mathrm{.} \mathrm{.} 83$

4.30 Frequenzverschiebung durch den $10 \mathrm{~nm}$ PdCuSi-Film . . . . . 84

4.31 Frequenzunterschied amorph-kristallin . . . . . . . . . . . 84

4.32 Einfluss der therm. Vorbehandlung auf das Spektrum . . . . . 86

5.1 Relaxationsspektrum von PdCuSi, gemessen mittels DMA . . 96

5.2 Komplexer Schermodul der $200 \mathrm{~nm}$ dicken PdCuSi-Schicht . . 96

5.3 Komplexer Schermodul einer ZrAlCu-Schicht . . . . . . . . . . 100

5.4 Schematische Darstellung der PEL . . . . . . . . . . . . . . . 101

5.5 Temperaturabhängigkeit des Schermoduls von PdNiP . . . . . 105

5.6 Schichtdämpfung auf glatten Substraten . . . . . . . . . . . 108

5.7 Glastemperatur und Kristallisationstemperatur . . . . . . . . 113

5.8 Schematische Darstellung eines $\beta$-Prozesses, Quadrupol . . . . 116

5.9 Schematische Darstellung eines $\beta$-Prozesses, Dipol . . . . . . . 117 
5.10 Der merging-Prozess als Funktion der Temperatur . . . . . . . 119

A.1 Weitwinkel-Röntgenmessung der $10 \mathrm{~nm} \mathrm{PdCuSi-Schicht} \mathrm{.} \mathrm{.} \mathrm{.} \mathrm{.} 127$

A.2 Kleinwinkel-Röntgenreflektivität der Probe DPO_DB_08 . . 128

A.3 Dämpfungsmessung von DPO_DB_05b auf rauem Substrat . 129

A.4 Dämpfungsmessung von DPO_DB_05c auf rauem Substrat . 130 


\section{Kapitel 1}

\section{Einleitung und Motivation}

\subsection{Ziel dieser Arbeit}

Die vorliegende Arbeit soll einen Beitrag zum Verständnis der mikroskopischen Prozesse in Gläsern leisten.

Gläser, insbesondere solche aus oxidischen Schmelzen, sind schon sehr lange bekannt und im Alltag allgegenwärtig. Auch viele der mittlerweile weit verbreiteten Kunststoffe sind von ihrer Struktur überwiegend amorph [1]. Noch wenig verbreitet, aber für einige Spezialanwendungen (z. B. Diebstahlsicherungen) bereits unersetzlich, sind spezielle Metalllegierungen, die ebenfalls in Form von Gläsern hergestellt werden können. Solche metallischen Gläser sind zumeist silbern glänzend, elektrisch leitfähig und teilweise auch sehr elastisch [2]. Allen Gläsern ist gemein, dass sich die Viskosität bis zum Glasübergang kontinuierlich sehr stark ändert [3]. Anschaulich zu verfolgen ist der Vorgang bei einem Glasbläser, der die hoch erhitzte Glasmasse nur in einem gewissen Temperaturbereich verformen kann. Dieser Übergang ist ein intensiv studiertes Gebiet der Festkörperphysik und wird in der aktuellen Forschung noch kontrovers diskutiert. Die Dynamik in Gläsern ist komplex und besteht nicht nur aus der einfachen Viskosität [4]. Es gibt Relaxationsmoden, die eine viel schnellere Dynamik aufweisen, von besonderem Interesse ist die sekundäre Relaxation ( $\beta$-Relaxation), auch Johari-Goldstein-Relaxation [5] genannt. Diese Relaxationsmode hat sich in Vorgängerarbeiten [6, 7] als ein universeller Bestandteil der Dynamik von metallischen Gläsern erwiesen und wird in der vorliegenden Arbeit weitergehend studiert. Dabei liegt der Schwerpunkt auf der Analyse des Einflusses von Längenskalen, experimentell über verschieden dicke Schichten des Probenmaterials realisiert.

Modelle zur Beschreibung der inneren Struktur von Gläsern postulieren dynamische Heterogenitäten als Ursache für die komplexe Dynamik [8]. Solche 
Heterogenitäten können in Computersimulationen $[9,10]$ bereits identifiziert werden, bestätigende experimentelle Messungen zu diesen Heterogenitäten fehlen bisher. In dieser Arbeit werden mechanische Messungen an dünnen Schichten bis zu einer minimalen Schichtdicke von $10 \mathrm{~nm}$ durchgeführt. Die Grundidee ist dabei, dass sich die mechanischen Eigenschaften der Proben deutlich ändern, wenn die Schichtdicke mit charakteristischen Längenskalen von Heterogenitäten vergleichbar ist. Gleichzeitig ändert sich bei immer dünneren Schichten das Aspektverhältnis, die Geometrie wird zunehmend zweidimensional. Ein weiterer Gegenstand dieser Experimente ist der zunehmende Einfluss von Grenzflächen und Oberflächen auf die Dynamik. Die Erforschung von Oberflächen- und Grenzflächeneffekten ist insbesondere für die „Nanotechnologie“, sowohl bei kristallinen also auch bei amorphen Materialien, von entscheidender Bedeutung [11]. Die mechanische Analyse der dünnen Schichten wird hier mit der Doppelpaddel-Oszillator (DPO) Methode [12] vorgenommen. Die Herstellung von amorphen PdCuSi-Schichten und auch deren mechanische Vermessung erfolgt im Ultrahochvakuum. Ein neuer Messaufbau zur Herstellung und Charakterisierung der Schichten wurde zu Beginn der Arbeit gebaut. Dabei ist gegenüber einem vorherigen Messsaufbau der zugängliche Temperaturbereich auf 200-750 K erweitert worden. Die Kühlmöglichkeit erlaubt die Erweiterung des bekannten Verlustspektrums metallischer Gläser zu tieferen Temperaturen, möglich durch die hohe Empfindlichkeit der DPO Messung.

Die Gliederung dieser Arbeit ist wie folgt aufgebaut:

Direkt im Anschluss an diesen Abschnitt werden einige Grundbegriffe der Glasphysik und Wesentliches zur Dynamik in Gläsern vorgestellt. Das Kapitel 1 schließt mit der Vorstellung aktueller Modelle zu Heterogenitäten und Längenskalen in Gläsern. Das Kapitel 2 dient einer kurzen Einführung der mechanischen Spektroskopie, nach der Vorstellung wichtiger Messmethoden dazu wird die DPO Methode erläutert, zusammen mit einer Abschätzung zu der Auflösungsgrenze. In Kapitel 3 werden alle experimentellen Details und Vorgehensweisen dargestellt. Die Messergebnisse in Kapitel 4 beginnen mit morphologischen Untersuchungen der Schichten. Es folgt eine kurze mechanische Charakterisierung der verwendeten Doppelpaddel und Messungen an dicken $\mathrm{PdCuSi}$-Schichten über den erweiterten Temperaturbereich. Danach werden die Ergebnisse zu den dünnen Schichten umfassend aufgezeigt. Eine Diskussion der Ergebnisse folgt im Kapitel 5, in Abschnitt 5.5.5 wird eine Modellvorstellung zu den beobachteten Größeneffekten vorgestellt. Abschließend fasst das Kapitel 6 die wichtigsten Ergebnisse noch einmal zusammen. 


\subsection{Amorphe Festkörper, Gläser, Schmelzen und Kristalle}

\subsubsection{Gläser, Glasübergang}

In dieser Arbeit werden Relaxationsprozesse metallischer Gläser untersucht. Die metallischen Gläser stellen eine Untergruppe der großen Materialklasse der Gläser dar, die alle unter den Überbegriff der amorphen Festkörper einzuordnen sind.

Zu Beginn dieser Arbeit sollen einige Begriffe kurz erläutert und durch Definitionen abgegrenzt werden: Der Kristall zeichnet sich durch eine periodische Anordnung der Atome aus, die sich idealisiert unendlich weit fortsetzt. Ein Stoff wird als amorph bezeichnet, wenn keine solche langreichweitige Ordnung vorliegt. Auch ein amorpher Stoff kann kurzreichweitige Ordnung aufweisen, jedoch nicht über einige Bindungslängen hinweg.

Gemäß einer gebräuchlichen Definition ist ein Körper dann fest, wenn seine dynamische Scher-Viskosität ${ }^{1} \eta$ den willkürlichen Wert von $10^{13,6} \mathrm{~Pa} \cdot \mathrm{s}$ übersteigt [3].

Der Begriff Glas wird in [3] folgendermaßen definiert: „Ein Glas ist ein amorpher Festkörper, der einen Glasübergang zeigt." Das schematische Bild für einen Glasübergang, ausgehend von einer Gleichgewichtsschmelze, ist in Abbildung 1.1 illustriert.

Beim Abkühlen einer Schmelze (Flüssigkeit) tritt i. A. bei der Schmelztemperatur $T_{m}$ der Übergang in den kristallinen Festkörper auf. Dieser Übergang vollzieht sich unter Volumenänderung und Freisetzung von latenter Wärme. Ist die Kristallisation beim Abkühlen z. B. durch Verhindern der Keimbildung unterdrückt, so setzt sich die thermodynamische Gleichgewichtslinie der Flüssigkeit fort. Man spricht von einer unterkühlten Schmelze. In diesem Temperaturbereich nimmt die Viskosität der Schmelze stark zu, bzw. die interne Relaxationszeit $\tau_{\text {int }}$ steigt an. Im Bereich der Glastemperatur, die in Abbildung 1.1 mit $T_{g}$ bezeichnet wird, findet man einen kontinuierlichen Übergang zu einem Glas. Je langsamer die Kühlrate, desto weiter kann die Probe in der unterkühlten Schmelze bleiben.

Untersuchungen zum Glasübergang werden zumeist bei konstanter Heizrate vorgenommen. Messungen der spezifischen Wärme liefern beim Glasübergang einen kontinuierlichen Anstieg der spezifischen Wärme in Form eines verbreiterten Sprungs. Auch alle weiteren physikalischen Größen ändern sich stetig bei diesem Übergang [13].

\footnotetext{
${ }^{1}$ Die dynamische Scher-Viskosität ist definiert durch: $\eta=\tau_{x} /\left(d v_{x} / d z\right)$, mit $\tau_{x}$ als Scherspannung in x-Richtung.
} 


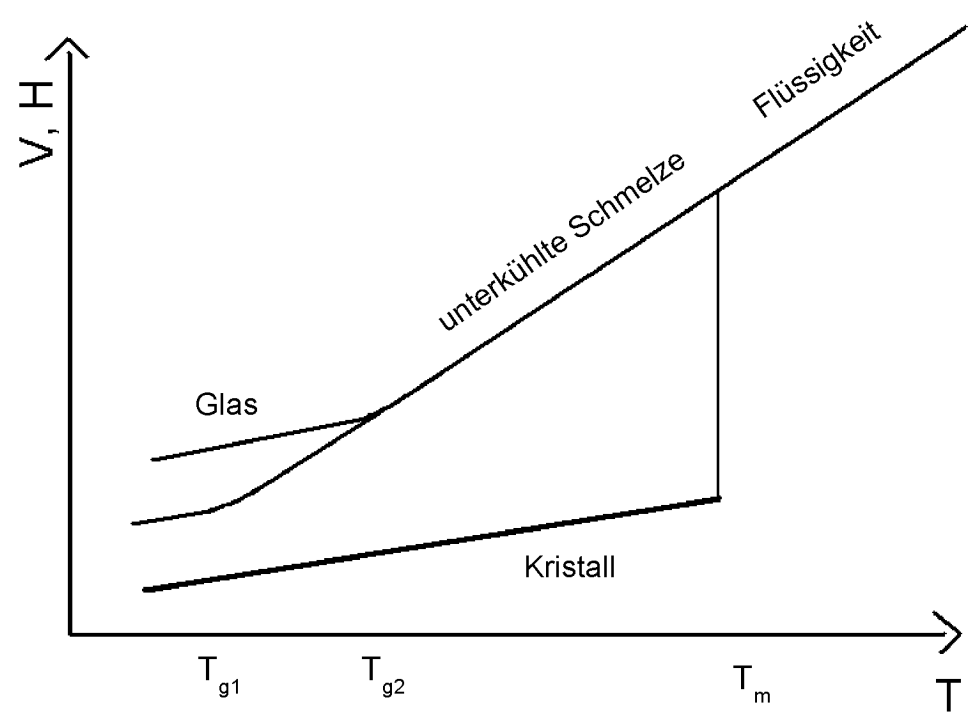

Abbildung 1.1: Schematische Darstellung eines Glasübergangs. Zeichnung erstellt nach [13].

Die Physik des Glasübergangs ist ein lang untersuchtes und bislang ungelöstes Problem der Festkörperphysik. In der Literatur werden umfangreich Theorien und Modelle diskutiert, es wird an gegebener Stelle darauf Bezug genommen.

\subsubsection{Metallische Gläser}

Während Gläser aus Oxiden, insbesondere aus Siliziumdioxid, bereits sehr lange als „Glas" allgemein bekannt sind, stellen metallische Gläser eine relativ neue Materialklasse dar. Seit 1954 kennt man die Eigenschaft von Metallen, bei sehr speziellen Kondensationsbedingungen amorph zu werden [14]. Massive Gläser aus speziellen Metalllegierungen wurden erstmals 1991 vorgestellt [15].

Metallische Gläser weisen Eigenschaften auf, die den kristallinen Ausgangsmaterialien ähneln, so ist ihre Erscheinung metallisch glänzend und auch die elektrische Leitfähigkeit ist typisch metallisch. Im Gegensatz zu oxidischen Gläsern sind metallische Gläser nicht so hart und spröde sondern dehnbar und in einem weiten Bereich elastisch verformbar. Die herausragenden mechanischen und auch magnetischen Eigenschaften können kristalline Legierungen weit übertreffen und führen zu einem gesteigerten Interesse der angewandten Forschung und der industriellen Anwendung. Einen Einblick in dieses Feld 
findet man z. B. in [16].

Aus der Sicht der Grundlagenforschung bieten metallische Gläser ebenfalls einige besondere Eigenschaften: Das Fehlen einer kristallinen Ordnung, in Verbindung mit den ungerichteten metallischen Bindungen, führt zu einem Körper der sich auf makroskopischen Skalen isotrop verhält. Diese Eigenschaft vereinfacht die Modellierung physikalischer Prozesse z. B. von Oberflächenmechanismen $[17,18]$. Das Verhalten kristalliner Materialien ist immer auch beeinflusst von Korngrenzen und Defekten. Solche Merkmale fehlen bei amorphen Metallen, makroskopisch verhalten sich die Proben (mit Ausnahme von Oberflächenbereichen) homogen ${ }^{2}$. Ausschlaggebend für die Wahl metallischer Gläser für diese Studie ist u. A. die einfache Struktur. Metallatome in einer ungeordneten Anordnung können durch harte Kugeln approximiert werden, die sich ähnlich wie Erbsen in einem Beutel verhalten. Das sogenannte dense random packing of hard spheres-Modell beschreibt harte Kugeln mit einem repulsiven Radius, der räumliche Überschneidung verhindert, in möglichst dichter Packung [19]. In einem solchen Modellbild gibt es nur wenige interne Freiheitsgrade, wodurch sich die Dynamik besser studieren lässt als bei komplexen organischen Glasbildnern.

\subsection{Dynamik in Gläsern}

Die Dynamik in Gläsern ist vielfältig, so treten zum einen eine Vielzahl an Prozessen auf, die beobachtet werden können, und zum anderen ändern sich die Zeitskalen der Dynamik beim Abkühlen der Schmelze über viele Größenordnungen. Viele Erkenntnisse über das Auftreten von internen (Relaxations)-Prozessen und ihrer Temperaturabhängigkeit sind mit dielektrischer Spektroskopie an organischen Glasbildnern gewonnen worden [4].

\subsubsection{Beiträge zur Dynamik}

Einen Überblick der beobachteten Prozesse in organischen Gläsern gibt die Abbildung 1.2.

Dargestellt ist der dielektrische Verlust über insgesamt 21 Größenordnungen in der Frequenz. Skizziert ist der Verlauf für zwei Temperaturen, um die Verschiebung des Spektrums mit der Temperatur anzudeuten. Es zeigen sich sehr breit verteilte Maxima, die Zuordnung zu mikroskopischen Prozessen wird im Folgenden besprochen. Die Beiträge werden nur kurz vorgestellt, da im Weiteren noch ausführlich auf den $\alpha$ - und $\beta$-Prozess für metallische Gläser eingegangen wird.

\footnotetext{
${ }^{2}$ Auf kleinen Längenskalen treten Heterogenitäten auf, siehe Abschnitt 1.4.
} 


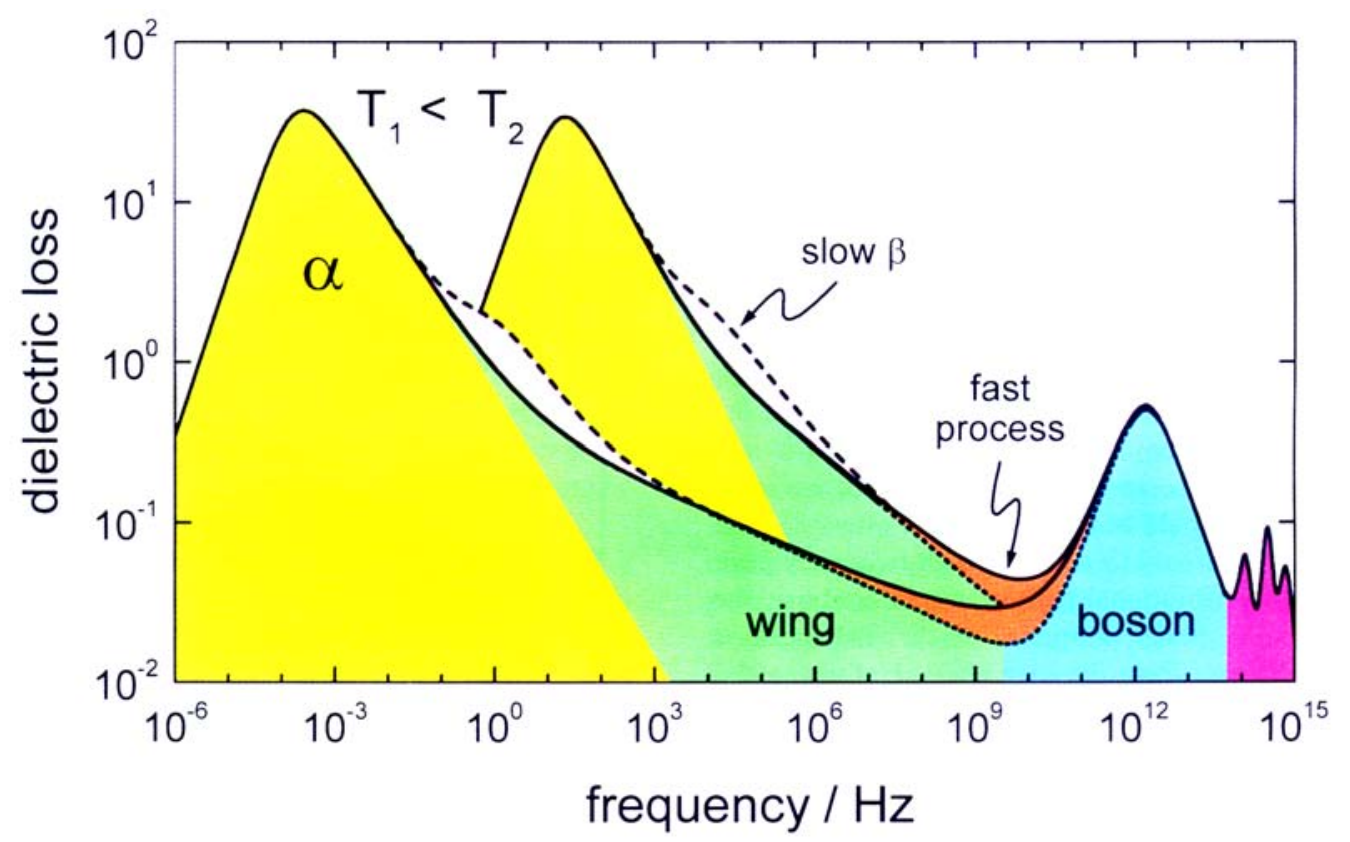

Abbildung 1.2: Schematische Übersicht des Relaxationsspektrums eines organischen Glasbildners [4].

Bei niedrigen Frequenzen dominiert der $\alpha$-Prozess, der den Verlust durch viskoses Fließen darstellt. Die Hochfrequenzflanke dieses Prozesses weist, je nach Material, einen erkennbaren slow $\beta$-Peak oder einen wing auf, der in dieser Arbeit zumeist nur als $\beta$-Prozess oder sekundärer Prozess bezeichnet wird. Die Trennung, bzw. das Zusammenlaufen der beiden Prozesse in Abhängigkeit von Frequenz und Temperatur wird unter dem Stichwort merging in Abschnitt 1.3.2 behandelt. Die Diskussion über die mikroskopische Ursache des $\beta$-Prozesses ist noch nicht abgeschlossen, jedoch werden kooperative Prozesse für diesen Prozess angeführt [20, 21]. Zu höherer Frequenz schließst sich ein Bereich mit fast konstantem Verlust, bzw. des fast process oder auch fast $\beta$-Prozesses an. Dieser Beitrag wird in der Modenkopplungstheorie [22] beschrieben und als „Rasseln“ der Atome in dem Käfig ihrer Nachbaratome erklärt. Bei noch höherer Frequenz erhebt sich der Boson-Peak, der mit elementaren Vibrationsmoden [23] oder lokalen strukturellen Konfigurationsänderungen in Einklang gebracht werden kann [24]. Abschließend sind im Spektrum organischer Glasdbildner Molekülschwingungen eingezeichnet, die bei optischen Frequenzen auftreten. 


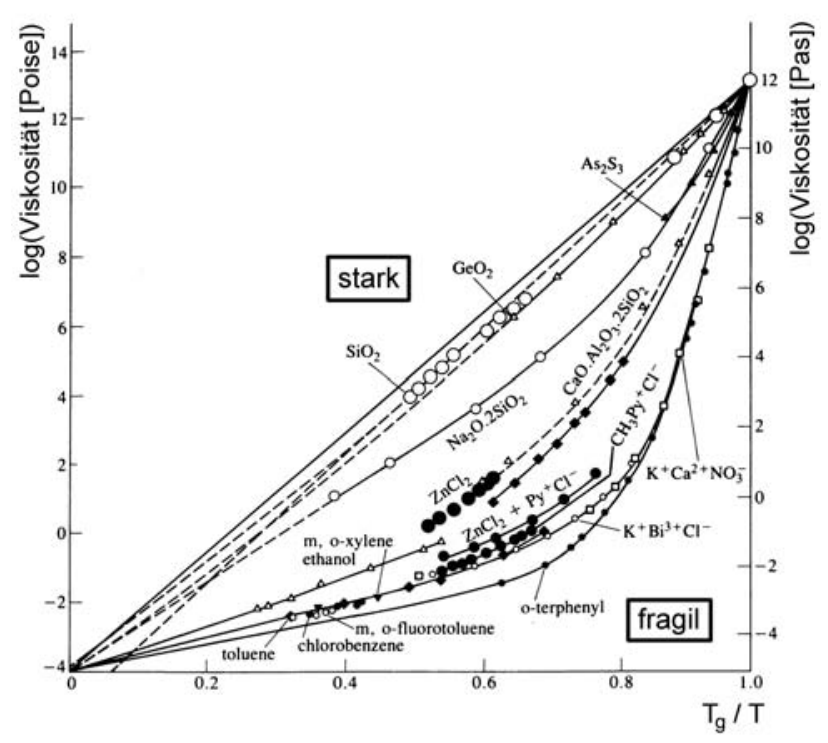

Abbildung 1.3: Viskositätsänderung von Glasbildnern oberhalb der Glastemperatur. Abbildung aus [6] nach [25]. Diese Glastemperatur wird bei $\eta=10^{12} \mathrm{~Pa} \cdot \mathrm{s}$ festgelegt.

\subsubsection{Temperaturabhängigkeit der Dynamik, „Merging““}

Die starke Temperaturabhängigkeit der Dynamik in Glasbildnern zeigt sich am deutlichsten in der Viskosität. Die Viskosität einiger Glasbildner steigt nahe der Glastemperatur schneller als exponentiell an. Einen Vergleich der Viskositätsänderungen von Glasbildnern gibt der Angell-Plot, der in Abbildung 1.3 dargestellt ist.

In dieser Darstellung, die die Viskosität oberhalb der Glastemperatur zeigt, ist die Temperaturskala mit der Glastemperatur skaliert worden. Diese Glastemperatur wird hier mit der Temperaur bestimmt, bei der die Viskosität den Wert $10^{12} \mathrm{~Pa} \cdot \mathrm{s}$ erreicht. Die Übersicht umfasst die Änderung der Viskosität über etwa 16(!) Größenordnungen. Aus dieser Darstellung folgt eine Unterteilung der Gläser in ,starke" und ,fragile" Gläser. Der Fragilitätsparameter $m$ gibt in dieser Darstellung die Steigung der Kurve bei $T_{g}$ an. Starke Gläser zeigen im Angell-Plot einen geraden Verlauf, der einem einfachen Arrhenius-Verlauf (Definition auf Seite 18) entspricht. Die in dieser Darstellung stärker gekrümmten Viskositätsänderungen der fragilen Gläser werden zumeist mit einer Vogel-Fulcher-Tammann Gleichung beschrieben [3]:

$$
\eta=\eta_{0} \exp \left(\frac{\alpha T_{0}}{T-T_{0}}\right), T_{0}<T
$$




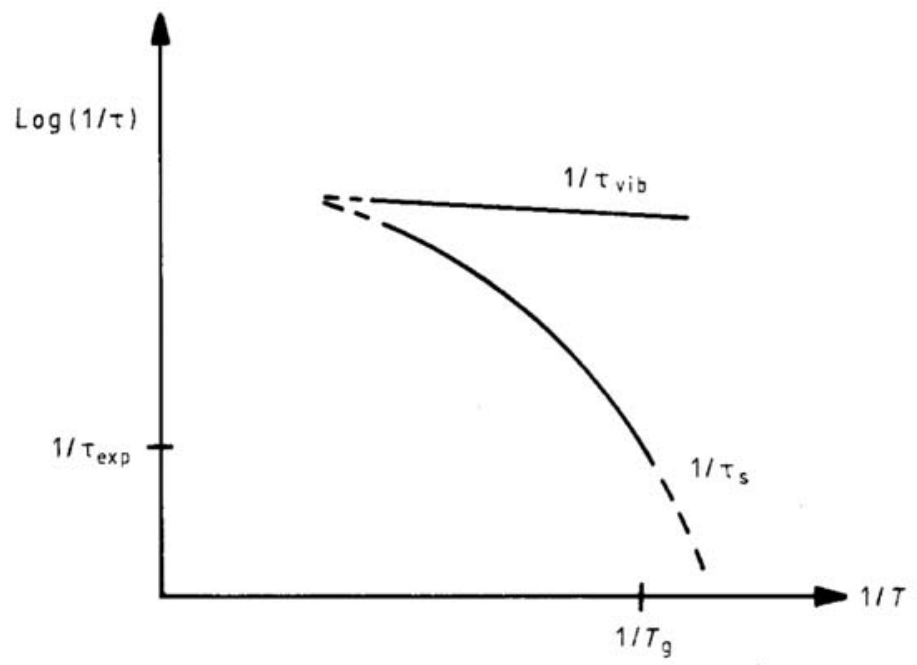

Abbildung 1.4: Illustration der Entkopplung von Zeitskalen der Vibrationen $\tau_{v i b}$ von strukturellen Zeitkonstanten $\tau_{s}$, Quelle: [28].

Die Variable $\alpha$ kann mit dem Fragilitätsparameter in Verbindung gebracht werden, i. A. stellen die Parameter $\alpha$ und $T_{0}$ nur Parameter der Anpassung dar. Kontrovers wird die Divergenz in der Viskosität gesehen, da diese mit der Relaxationszeit $\tau_{\text {int }}$ für Scherspannung verknüpft ist:

$$
\tau_{\text {int }}=\frac{\eta}{G_{\infty}}
$$

Dabei bezeichnet $G_{\infty}$ das instantane Schermodul [3]. Eine Divergenz in $\eta$ führt so zu einer divergierenden Zeitskala bei $T_{0}$. Da dies einen Phasenübergang indiziert, gibt es aktuelle Publikationen, in denen die physikalische Relevanz der Vogel-Fulcher-Tammann Gleichung angezweifelt wird [26, 27]. In einer Studie von J. C. Dyre et al. zeigt eine Avramov Gleichung mit zwei Fitparametern, ohne Divergenz, ebenfalls gute Anpassung an Messdaten [27]:

$$
\tau(T)=\tau_{0} \exp \left(\frac{B}{T^{n}}\right)
$$

Die Temperaturabhängigkeit anderer Moden zeigt sich deutlich geringer als die der Viskosität. In einer Arbeit von J. Jäckle wird eine Entkopplung von strukturellen (diffusiven) Relaxationszeiten gegenüber Zeitskalen der Vibration beim Abkühlen der Schmelze gezeigt [28]. Diese Entkopplung ist in Abbildung 1.4 schematisch gezeigt und wird auf die schnelle Verlangsamung der strukturellen Prozesse zurückgeführt. 


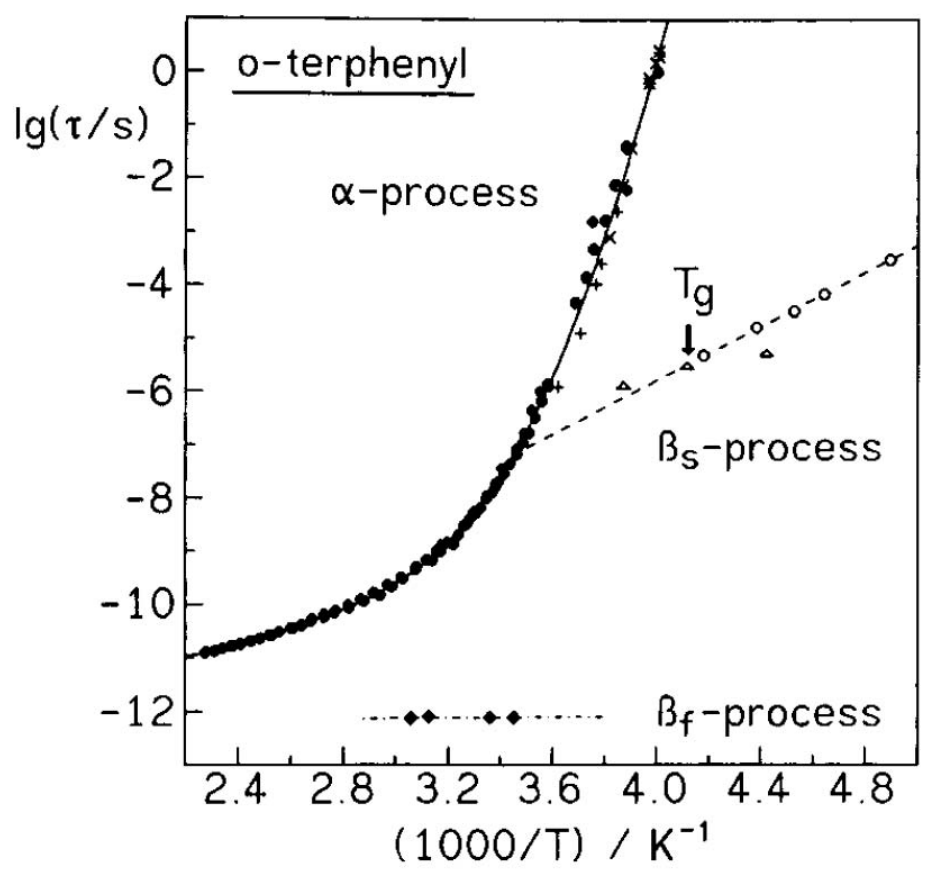

Abbildung 1.5: Relaxationszeiten von o-Terphenyl, bestimmt aus verschiedenen Messungen [29]. Dargestellt ist der Verlauf vom $\alpha$-Prozess, langsamen $\beta$-Prozess $\left(\beta_{s}\right)$ und auch vom schnellen $\beta$-Prozess $\left(\beta_{f}\right)$.

Eine umfangreiche Studie an o-Terphenyl, einem organischen Glasbildner, unter Verwendung einer Vielzahl an Messmethoden (siehe [29]), liefert ein möglichst vollständiges Relaxationsspektrum, wie es in Abbildung 1.5 gezeigt ist.

Dabei verläuft der $\alpha$-Prozess mit einem Vogel-Fulcher-Tammann-Verhalten, der slow $\beta$-Prozess $\left(\beta_{s}\right)$ zeigt eine deutlich geringere Temperaturabhängigkeit und ist mit einem Arrhenius-Verhalten zu beschreiben. Mit Neutronenstreuexperimenten kann der Verlauf für den fast $\beta$-Prozess $\left(\beta_{f}\right)$ bestimmt werden. Dieser zeigt sehr viel kürzere Zeitkonstanten mit kaum erkennbarer Temperaturvariation [30]. Zu bemerken ist insbesondere der Schnittpunkt der $\alpha$ und $\beta_{s}$-Linien. Bei dieser Temperatur tritt beim Abkühlen ein separater $\beta$-Prozess auf. Umgekehrt wird das erwartete Zusammenlaufen der beiden Prozesse bei dieser Temperatur beim Heizen als merging bezeichnet. Je nach Frequenz und Temperatur sind die beiden Prozesse also deutlich getrennt oder laufen zusammen. Der Schnittpunkt liegt hierbei deutlich oberhalb der (üblichen) Glastemperatur. Extrapoliert man die Messdaten des schnellen $\beta$-Prozesses $\mathrm{zu}$ hohen Temperaturen, so ist auch hier ein Zusammenlaufen mit dem $\alpha$ Prozess zu erwarten, so wie in Abbildung 1.4 schematisch skizziert. 


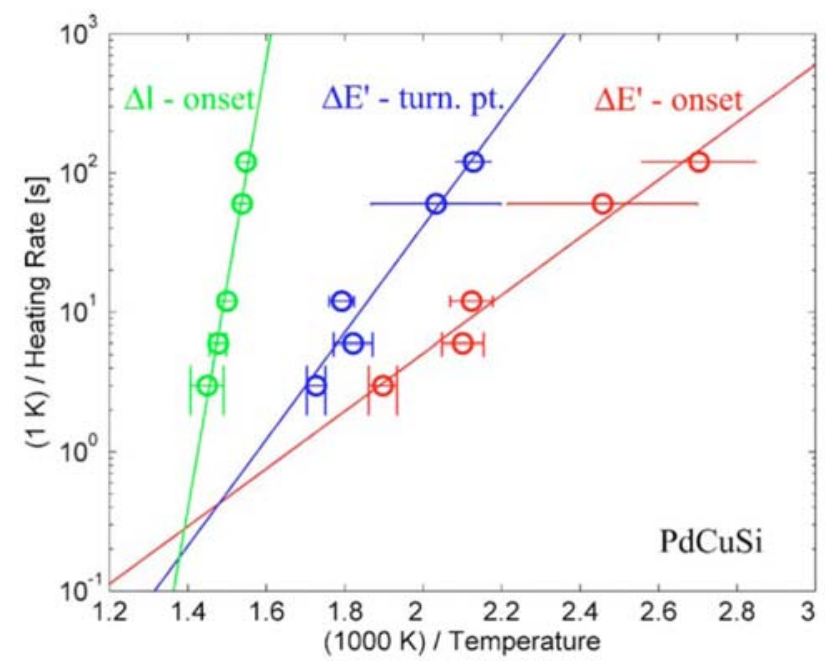

Abbildung 1.6: Aktivierungsplot für das ratenabhängige viskose Fließen (grün), Alterung durch einen $\beta$-Prozess (rot bzw. blau). Durch lineare Extrapolation kann eine Merging-Temperatur bestimmt werden [31].

Für metallische Gläser ist die vollständige Untersuchung der Relaxationszeiten über diesen großen Temperaturbereich bisher nicht möglich, da diese oberhalb der Glastemperatur die starke Tendenz zur Kristallisation aufweisen. Zudem können metallische Gläser wegen ihrer hohen Leitfähigkeit nicht mittels dielektrischer Verlustspektroskopie vermessen werden. Die mechanische Analyse ist technisch bedingt zunächst eingeschränkter in den Frequenzen, dennoch können Teile des Relaxationszeit-Temperatur-Diagramms vermessen werden. In seiner Dissertation konnte J. Hachenberg deutlich verschiedene Temperaturabhängigkeiten für den $\alpha$-Prozess und einen $\beta$-Prozess ermitteln [7]. Hierzu wurden heizratenabhängig die Temperaturen des einsetzenden $\beta$-Prozesses mit dem Einsetzen einer Alterung der Proben (siehe Abschnitt 1.3.3) und der $\alpha$-Prozess mit dem Beginn des Fließens der Proben bestimmt. Das Ergebnis ist in Abbildung 1.6 in einem Aktivierungsplot gezeigt.

Diese Darstellung erlaubt zum einen die Bestimmung von Aktivierungsenergien und zum anderen kann durch lineare Extrapolation der Messdaten eine Merging-Temperatur bestimmt werden, bei der die beiden Prozesse zusammenlaufen, die deutlich über der Glastemperatur liegt [31].

Ausgehend von einer Schmelze der glasbildenden Legierung über dem Schmelzpunkt, kann die diffusive Dynamik mittels inelastischer Neutronenstreuung beim Unterkühlen beobachtet werden [32]. Bei hoher Temperatur können die so bestimmten Diffusionskonstanten mit der Stokes-Einstein Relation 
$D=\frac{K_{B} T}{6 \pi \eta r}$ beschrieben werden, beim Abkühlen trennen sich gemessene Diffusion und die Viskosität: Es werden größere Diffusionskonstanten gemessen, als nach der Stokes-Einstein Relation aus der Viskosität vorhersagt. Diese Abweichungen werden ebenfalls mit einem Aufspalten der Dynamik in einen $\alpha$ - und einen $\beta$-Prozess unterhalb einer kritischen Temperatur erklärt [21].

\subsubsection{Alterungen}

Eine besondere Variante der Dynamik in Gläsern ist die Alterung, auch aging genannt. Beim Abkühlen einer glasbildenden Schmelze können die Atome bzw. Moleküle, bedingt durch die schnell anwachsende strukturelle Zeitskala, ihre Konfiguration nicht schnell genug in die Gleichgewichtskonfiguration umwandeln, die der aktuellen Temperatur entspricht. Etwa bei der Glastemperatur fällt die Probe, kinetisch bedingt, aus dem thermodynamischen Gleichgewicht einer Flüssigkeit. Wird die Probe bei einer Temperatur nahe der Glastemperatur ausgelagert, so treten strukturelle Relaxationen auf, die die Eigenschaften des Glases stark verändern können. Für den Fall der metallischen Gläser sind Änderungen u. A. bzgl. Volumen, spezifischer Wärme, Elastizitätsmodul, innerer Reibung, Diffusivität, Versprödung, und auch in magnetischen Eigenschaften bekannt [2]. Hervorzuheben sind hierbei besonders die Versprödung, da diese ein technologisches Problem für Anwendungen darstellt und die Änderungen in Modul und innerer Reibung, da diese durch mechanische Spektroskopie auch in dieser Arbeit untersucht werden. In einer Arbeit von Lunkenheimer et. al. wird für einige organische Glasbildner die Dynamik der Alterung mittels dielektrischer Spektroskopie untersucht. Demnach kann die Abnahme des Verlustes (Dämpfung) mit einer angepassten Kohlrausch-William-Watts Funktion beschrieben werden (Gleichung 2.18), die Dynamik erweist sich für alle Prozesse durch die strukturelle Zeitabhängigkeit determiniert [33].

Messungen zur Alterung in metallischen amorphen Bändern von J. Hachenberg zeigen einen Anstieg des Elastizitätsmoduls bereits weit unterhalb von der Glastemperatur. Dieser Anstieg kann nicht mit einem $\alpha$-Prozess erklärt werden [7], worauf in [31] die Unterscheidung in $\alpha$-aging und $\beta$-aging vorgeschlagen wird. Der Vorgang der $\beta$-Alterung zeigt sich bei Auslagerungen deutlich unter $T_{g}$ bezüglich der Temperaturabhängigkeit als reversibel [34]. 


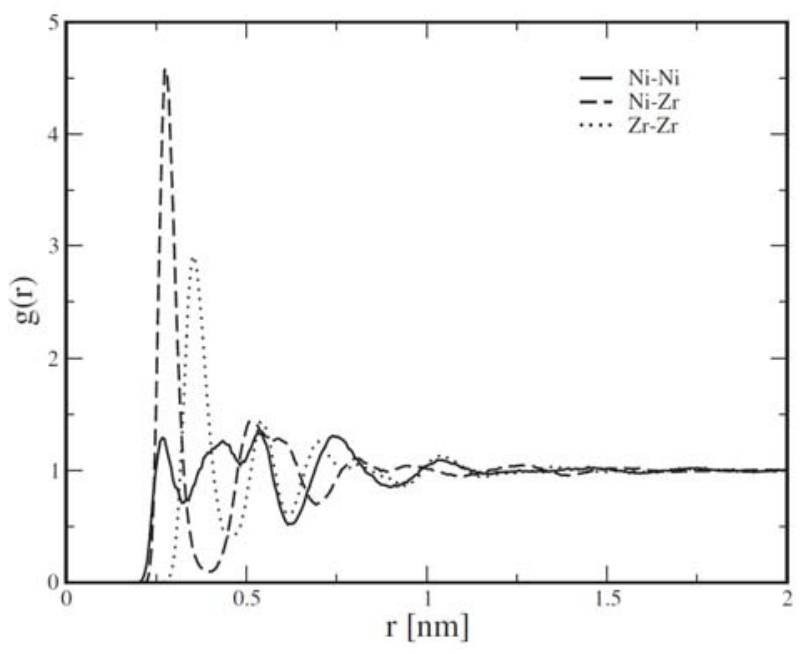

Abbildung 1.7: Partielle radiale Strukturfunktion aus einer Simulation an $\mathrm{Ni}_{0,5} \mathrm{Zr}_{0,5}$ bei $700 \mathrm{~K}[37]$.

\subsection{Heterogenitäten und Längenskalen in Gläsern}

Die Besonderheiten in der Dynamik von Gläsern im Vergleich zu einfachen Flüssigkeiten oder Kristallen werden vielfach mit lokalen dynamischen Heterogenitäten erklärt. Die Zahl der Studien und Modelle zu diesem aktuellen Themenkomplex ist sehr umfangreich. Einen Übersichtsartikel zu diesem Thema stellt [35] dar, eine Sammlung von Publikationen zu diesem Thema ist außerdem in [36] zu finden.

Die hier vorgestellten Experimente haben das Ziel einen experimentellen Zugang zum Studium kleiner Längenskalen, realisiert durch sehr dünne Schichten, zu ermöglichen, daher werden wichtige Grundbegriffe als Motivation dieser Arbeit und als Basis der später folgenden Diskussion an dieser Stelle vorgestellt:

Die kleinste natürliche Längenskala in Gläsern stellt der Abstand nächster Nachbarn dar, im Fall von harten Kugeln mit Radius $R$ ist der wahrscheinlichste radiale Abstand $r=2 \cdot R$. Ein genaues Bild der Verteilung von Atomen zueinander wird mit der radialen Strukturfunktion $g(r)=\rho(r) / \rho_{0}$ angegeben, wobei $\rho_{0}$ die mittlere Dichte beschreibt. Die radiale Strukturfunktion kann aus Computersimulationen, Streuexperimenten und aus Strukturmodellen berechnet werden und erlaubt einen Vergleich von Modellvorstellungen und Messdaten [3]. Beispielhaft soll das metallische Glas Ni-Zr aus einer Simulationsstudie von Teichler et al. gezeigt werden [37]. 
In Abbildung 1.7 ist die radiale Strukturfunktion für die verschiedenen Elementkombinationen gezeichnet, man erkennt insbesondere die verschiedenen Radien der Elemente beim ersten Minimum bzw. Maximum der Kurven. Außer dem offensichtlichen ersten Maximum bei $r=R_{i}+R_{j}$, treten in $\mathrm{g}(\mathrm{r})$ noch weitere schwächer ausgeprägte und breitere Maxima auf, die weniger scharf definierten Abständen zu weiter entfernten Atomen entsprechen. Dieses wird als short-range order oder medium-range order bezeichnet und ist durch eine charakteristische Anordnung der Kugeln zueinander auf einer Längenskala bis etwa $20 \AA$ gegeben [3]. Eine lokale Packung durch Anordnung zu einem Ikosaeder aus 13 oder 55 Atomen ist energetisch besonders günstig. Die 5-zählige Symmetrie der Ikosaeder verhindert jedoch eine dreidimensionale Raumerfüllung [3]. Für die in Abbildung 1.7 gezeigte lokale Ordnung wird die bevorzugte Lage der Nickel-Atome in Löchern der, von Zr-Atomen gebildeten, trigonalen Prismen angeführt [37].

In der Modellierung dynamischer Eigenschaften von Gläsern stellen dynamische Heterogenitäten ein entscheidendes Merkmal dar. Bereits 1965 wurde von G. Adam und J. H. Gibbs ein Modell der cooperative rearranging regions vorgeschlagen [38]. In diesem Modell kann die starke Viskositätsänderung der Gläser mit einer Größenzunahme dieser Bereiche erklärt werden. Auf eine Veröffentlichung von A. S. Argon geht die Idee von shear transformation zones (STZs) zurück [8]. A. S. Argon beschreibt diese Bereiche als sphärisch mit etwa 5 Atomen im Durchmesser. Durch deren Reorientierung wird Scherspannung plastisch abgebaut. In dem cooperative shearModell (CSM) von K. Samwer und W. L. Johnson wird die Zahl der Atome in einem solchen STZ zu 100 Atomen für ein metallisches Glas vorhergesagt [39]. In einer Molekulardynamik-Studie von M. Zink bestätigt sich diese Vorhersage, zudem konnte ein typischer STZ-Durchmesser von 1,5 nm bestimmt werden [10]. Es zeigen sich bei den dort gescherten Simulationszellen, dass die sphärischen, kooperativ verformten Bereiche durch „string“-artige Gebilde verknüpft sind.

Teichler et al. konnten ,string“"-artige Ketten als mikroskopische Ursache der schnellen Relaxationsprozesse identifizieren [9]. Dabei bewegen sich Atome ebenfalls kooperativ in einer kettenartigen Bewegung. Diese Prozesse werden als teilweise reversibel beobachtet [37].

Experimentell gibt es an metallischen Gläsern bisher keine Messungen in diesem Längenbereich. Damit fehlt die Verknüpfung zwischen diesen Simulationsstudien und experimentellen Resultaten.

Für organische Glasbildner und insbesondere Polymere gibt es aktuelle Experimente zum Verhalten bei kleinen Längenskalen. Dabei liegt der Schwerpunkt zumeist auf dem Glasübergang bzw. der Frage einer $T_{g}$-Verschiebung. Hier gibt es Studien an dünnen Schichten und an Füllung in nanoporösen 

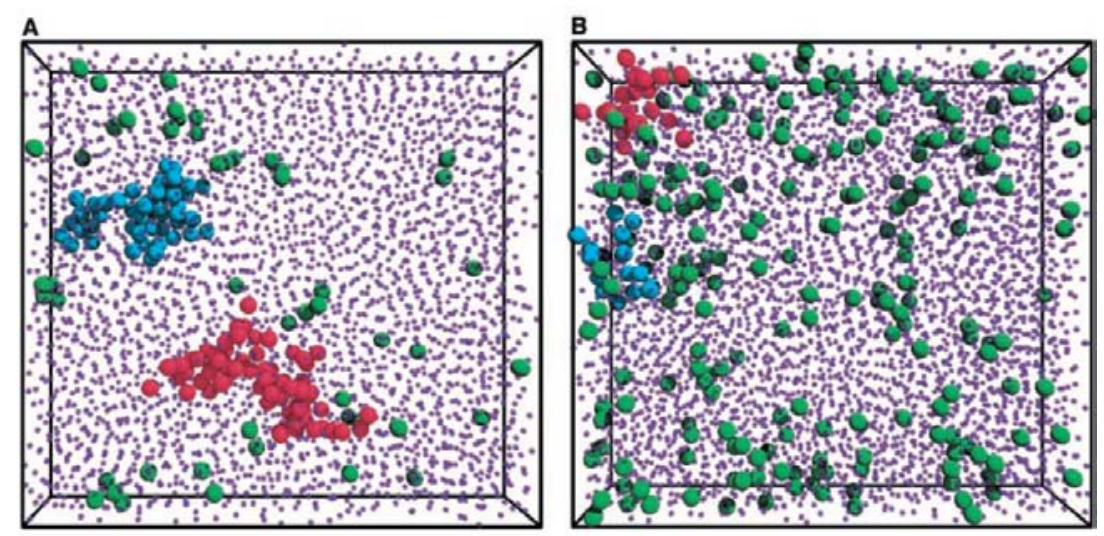

Abbildung 1.8: Darstellung der kolloiden Partikel mit großer Bewegung in einem festen Zeitintervall. In A ist eine als unterkühlt bezeichnete Probe und in B der glasartige Zustand repräsentiert, Quelle: [44].

Materialien [40, 41, 42, 43].

Mikroskopische Aufnahmen, mittels konfokaler Mikroskopie an Kolloid-Systemen, zeigen dreidimensionale, dynamische Strukturen, die den Heterogenitäten in den vorher zitierten Molekulardynamik-Simulationen qualitativ entsprechen [44]. Man beobachtet in der „unterkühlten“ Probe clusterförmige Bewegungen, die einem langsamen $\alpha$-Prozess entsprechen und im glasartigen Bereich schnelle kettenartige Bewegungen auf einer $\beta$-Zeitskala (Abb. 1.8). Kolloide werden wie metallische Gläser ebenfalls mit harten Kugeln approximiert, jedoch ist die Längenskala erheblich größer $(R \approx 1 \mu \mathrm{m})$.

Motiviert von diesen Beobachtungen stellt sich die Frage nach dem Verhalten für zweidimensionale Systeme. Auch bzgl. dieser Fragestellung können Experimente mit sehr dünnen (unendlich ausgedehnten) Filmen zu weiteren Erkenntnissen führen. 


\section{Kapitel 2}

\section{Mechanische Spektroskopie}

In diesem Kapitel sollen zuerst die wesentlichen Grundlagen der mechanischen Spektroskopie erläutert werden. Alle hierzu angegeben Formeln und Rechnungen sind aus [45] entnommen und können dort detailliert nachvollzogen werden. Es folgt ein Abschnitt über Relaxationsspektren. Anschließend werden die wichtigsten Techniken exemplarisch vorgestellt und verglichen. Der Schwerpunkt liegt auf der hier verwendeten Doppelpaddel-Oszillator Technik. Nach einer Erläuterung dieser Methodik in Abschnitt 2.4 folgt eine Abschätzung der Messempfindlichkeit in Abschnitt 2.4.4.

\subsection{Allgemeine Begriffe der mechanischen Spektroskopie}

Mechanische Spektroskopie findet breite Anwendung bei einer Vielzahl von Fragestellungen in der Festkörperphysik, Materialphysik und Materialwissenschaften bzw. auch im Bereich der Ingenieurwissenschaften. Dabei wird stets das Verformungsverhalten eines Festkörpers auf von außen angelegte mechanische Krafteinwirkung untersucht.

Im allgemeinen Fall wird der Zusammenhang zwischen angelegter Spannung $\sigma$ und Verformung $\epsilon$ durch ein verallgemeinertes Hooksches Gesetz beschrieben:

$$
\sigma_{i j}=C_{i j k l} \cdot \epsilon_{k l}
$$

Für isotrope Materialien genügen die einfachen Definitionen des Elastizitätsmoduls $E$ und des Schermoduls $G$ gemäß:

$$
\sigma=E \cdot \epsilon
$$




$$
\tau=G \cdot \gamma
$$

Dabei stellt $\tau$ die Scherspannung und $\gamma$ die Scherdehnung dar. Die beiden bisher definierten Module setzen Volumenerhaltung bei der Deformation voraus. Ebenfalls kann der so genannte Kompressionsmodul oder Bulkmodul B durch den Druck $p$ für hydrostatische Volumenänderung $\Delta V$ des Volumens $V$ definiert werden:

$$
p=-B \frac{\Delta V}{V}
$$

Da für einen isotropen Festkörper nur zwei unabhängige elastische Konstanten existieren, gilt ebenfalls:

$$
\frac{3}{E}=\frac{1}{G}+\frac{1}{3 B}
$$

Alle rein elastischen Beschreibungen gelten streng nur für sehr kleine Dehnungen und für kurze Zeiten. Bei größeren Dehnungen treten zuerst anelastisches und anschließend plastisches Verformungsverhalten auf. Bei längeren Zeiten treten ebenfalls Abweichungen vom rein elastischen Verhalten auf. Man bezeichnet diese Prozesse als Fließen oder auch als Kriechen.

\subsubsection{Dynamisch-mechanische Messungen}

Um interne Relaxationen zu vermessen, kommen dynamisch-mechanische Messungenmethoden zum Einsatz. Dabei wird eine periodische Spannung angelegt:

$$
\sigma=\sigma_{0} \sin (\omega t)
$$

Die Dehnung folgt dieser Spannung mit einer Phasenverschiebung $\Phi$, die durch Relaxationszeiten bedingt ist.

$$
\epsilon=\epsilon_{0} \sin (\omega t-\Phi)
$$

Nun ist es praktisch einen komplexen, frequenzabhängigen Elastizitätsmodul $E(\omega)$ einzuführen ${ }^{1}$.

$$
E(\omega)=E^{\prime}(\omega)+i E^{\prime \prime}(\omega)
$$

Der Speichermodul $E^{\prime}(\omega)$ beschreibt dabei den elastischen Anteil des EModuls, für den das Verhältnis $\sigma / \epsilon$ in Phase mit der Anregung ist. Der Verlustmodul $E^{\prime \prime}(\omega)$ beschreibt den dazu senkrechten Anteil, der durch dissipative Prozesse bedingt ist.

\footnotetext{
${ }^{1}$ Für periodische Scherdeformationen wird ein komplexer Schermodul völlig analog hierzu definiert.
} 
Für den komplexen Elastizitätsmodul ${ }^{2}$ gelten folgende wichtige Relationen:

$$
\begin{aligned}
& |E|=\sqrt{E^{\prime 2}+E^{\prime \prime} 2} \\
& \tan \Phi(\omega)=\frac{E^{\prime \prime}(\omega)}{E^{\prime}(\omega)}
\end{aligned}
$$

Mit dieser Relation ist $\tan \Phi$ ein direktes Maß für Dämpfung. Bei einer Schwingungsperiode dissipiert die Energie $\Delta W$ der maximal gespeicherten elastischen Energie $W$ nach folgender Gleichung:

$$
\frac{\Delta W}{W}=2 \pi \tan \Phi
$$

In dieser Arbeit wird als Maß für Dämpfung häufig der inverse Qualitätsfaktor $Q^{-1}$ verwendet. Für kleine Dämpfungen ist dieser identisch mit $\tan \Phi$.

$$
\tan \Phi \approx Q^{-1}
$$

\subsection{Relaxationsmessungen}

Zur Vermessung von internen mikroskopischen Prozessen in Festkörpern können mechanische oder auch dielektrische Spektroskopiemethoden angewendet werden. Dabei testet man das Verhalten auf variable Temperatur, Heizrate, Spektroskopiefrequenz, Anregungsstärke oder Dehnungsrate, um daraus Aktivierungsenergien oder Aktivierungsvolumina, bzw. interne Relaxationszeiten zu bestimmen. Eine Erläuterung dieser Zusammenhänge in allgemeiner Form soll hier nicht erfolgen, detaillierte Beispiele für solche Analysen finden sich z. B. in [45].

In dieser Arbeit liegt der Schwerpunkt auf der mechanischen Verlustspektroskopie. Ein solcher mechanischer Verlust ist bei dynamischen Messungen dann maximal, wenn ein interner, z. B. thermisch angeregter, Prozess mit einer charakteristischen Zeitkonstante $\tau$ auftritt, die mit der mechanisch angelegten Frequenz $\omega$ übereinstimmt.

$$
\omega \tau=1
$$

Für glasbildende Systeme sind charakteristische Zeitskalen und interne Dynamik, soweit bekannt, bereits in Kapitel 1.3 erläutert.

\footnotetext{
${ }^{2}$ Anmerkung: Setzt man den komplexen Elastizitätsmodul in eine Wellengleichung ein, so bedingt $E^{\prime}$ den propagierenden Anteil der Schwingung und $E^{\prime \prime}$ den exponentiellen Abfall der Schwingungsamplitude.
} 
Um charakteristische Zeiten und die Anregungsstärken der zugrunde liegenden Prozesse zu vermessen, gibt es im Wesentlichen zwei verschiedene Vorgehensweisen:

1. Die direkte Methode ist die Variation der Spektroskopiefrequenz bei konstanter Probentemperatur. Insbesondere bei dielektrischer Spektroskopie kann die Messfrequenz über 18 Größenordnungen variiert werden [4]. Aus der Auftragung von Energieverlust über Frequenz können interne Relaxationszeiten direkt abgelesen werden. Bei mechanischen Messungen ist dies aus technischen Gründen zumeist nicht möglich, weswegen überwiegend Variante 2. verwendet wird.

2. Eine grundsätzlich gegensätzliche Vorgehensweise ist es, eine konstante Spektroskopiefrequenz zu verwenden und die Temperatur der Probe $\mathrm{zu}$ variieren. Im Falle von thermisch getriebenen Relaxationsprozessen, die zumeist durch ein Arrhenius-Gesetz mit einer Aktivierungsenthalpie $H_{a c t}$ beschreibbar sind, ändert sich durch das Heizen oder Kühlen die interne Zeitskala:

$$
\tau(T)=\tau_{0} \exp \left(\frac{H_{a c t}}{k_{B} T}\right)
$$

Auch hierbei wird resonante Energieabsorption beobachtet, wenn $\omega \tau(T)=1$ gilt.

Um Spektren, die mit Methode 2 gewonnen worden sind, mit denen aus Methode $1 \mathrm{zu}$ vergleichen, wird das so genannte Zeit-Temperatur-Superpositionsprinzip verwendet, welches prinzipiell auf Gleichung (2.14) basiert und in [6] ausführlich beschrieben ist.

In Gläsern wird die Temperaturabhängigkeit der charakteristischen Zeitskala häufig mit einer Vogel-Fulcher-Tammann-Funktion beschrieben (Gl. (1.1)), die bereits im Kapitel 1.3.2 auf Seite 7 eingeführt worden ist.

\subsubsection{Debye-Relaxation}

Eine Debye-Relaxation ist das Standard-Beispiel einer Relaxation mit nur einer internen Zeitskala. Allgemeinere Spektren stellen zumeist eine Kombination mehrerer Debye-Spektren dar. Deswegen wird diese einmal exemplarisch für den Fall eines standard linear solid - Modells beschrieben.

Ein solches Modell ist in Abbildung 2.1 dargestellt. Die Parallelschaltung einer rein elastischen Feder mit einer Serie von Dämpfungstopf und Feder repräsentiert zum einen einen instantanen elastischen Anteil und zum anderen einen zeitabhängigen Anteil der Kraftantwort auf eine Verformung. 


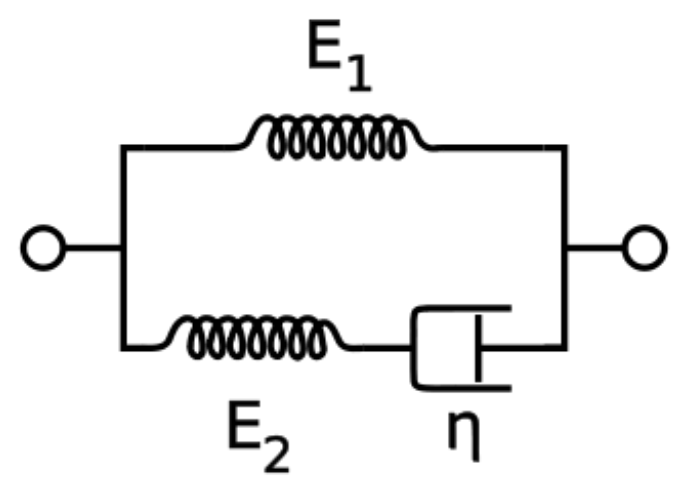

Abbildung 2.1: Ersatzschaltbild eines standard linear solid - Modells mit zwei Federn und einem Dämpfungstopf, Quelle: [46].

Diese Kraftantwort ist gegeben durch $\sigma_{2}=\eta \dot{\epsilon}$, mit $\eta$ als Viskosität des Dämpfungstopfes. Der zeitabhängige Anteil relaxiert exponentiell mit einer charakteristischen Zeitkonstante $\tau_{\epsilon}$,

$$
\tau_{\epsilon}=\frac{\eta}{E_{2}}
$$

Für ein solches Modell kann zu einer dynamischen Deformation der komplexe elastische Modul $E(\omega)$ hergeleitet werden [45]:

$$
\begin{gathered}
E^{\prime}(\omega)=\left(E_{1}+E_{2}\right)-E_{2} \frac{1}{1+\omega^{2} \tau_{\epsilon}^{2}} \\
E^{\prime \prime}(\omega)=E_{2} \frac{\omega \tau_{\epsilon}}{1+\omega^{2} \tau_{\epsilon}^{2}}
\end{gathered}
$$

Die Gleichungen (2.16) und (2.17) werden als Debye-Gleichungen bezeichnet. Abbildung 2.2 zeigt eine graphische Darstellung für das numerische Ergebnis für $E_{1}=5, E_{2}=10$ und $\tau=10$. Man erkennt für den Speichermodul $E^{\prime}$ einen stufenförmigen Anstieg mit maximaler Steigung bei $\omega \tau(T)=1$. Der Verlustmodul $E^{\prime \prime}$ ist bei dieser Frequenz ebenfalls am größten. Er nimmt symmetrisch um diese Frequenz stark ab, wobei sich in der logarithmischen Auftragung eine Gerade mit Steigung \pm 1 ergibt.

\subsubsection{Allgemeine Relaxationsspektren}

Experimentelle Relaxationsspektren an ungeordneten Systemen sind mit der einfachen Debye-Relaxation vielfach nicht gut beschreibbar. Eine DebyeRelaxation im Frequenzraum enspricht im Zeitraum einer rein exponentiellen 


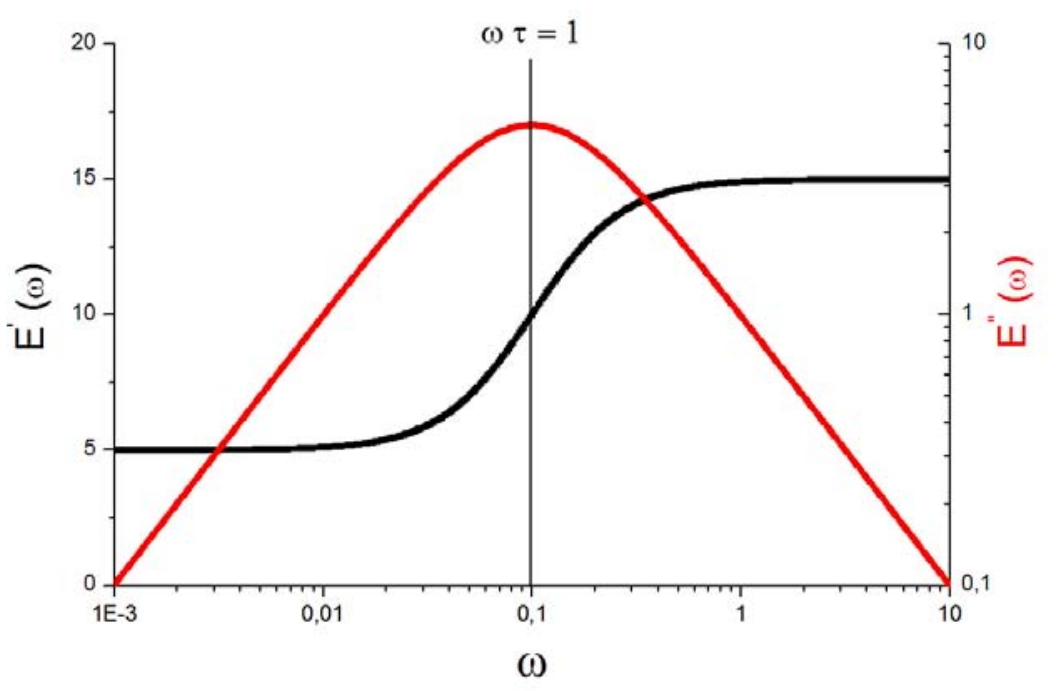

Abbildung 2.2: Darstellung einer Debye-Relaxation aus einer exemplarischen Berechnung $\left(E_{1}=5, E_{2}=10, \tau=10\right)$.

Relaxation. In Gläsern tritt jedoch ein nicht exponentieller Zeitverlauf auf. Die Relaxation z. B. einer Scherspannung kann besser mit einer KohlrauschWilliam-Watts-Funktion beschrieben werden [3]:

$$
\Phi(t)=\exp \left[-(t / \tau)^{\beta}\right], 0<\beta<1
$$

Für den Frequenzraum wurde 1967 eine empirische Relaxationsfunktion von Havriliak und Negami vorgeschlagen, die auch nicht symmetrische Relaxationsspektren und andere Steigungen im Imaginärteil der Relaxationsfunktion anpassen kann.

Für eine allgemeine Havriliak-Negami (HN) Funktion $\chi(\omega)_{H N}$ lautet die Definition in komplexer Schreibweise [47, 6]:

$$
\chi^{*}(\omega)_{H N}=\chi_{\infty}+\left(\chi_{s}-\chi_{\infty}\right) \frac{1}{\left[1+\left(i \omega \tau_{H N}\right)^{\alpha}\right]^{\gamma}}
$$

Dabei beschreibt $\chi_{s}$ den statischen Grenzwert für niedrige Frequenzen und $\chi_{\infty}$ den Grenzwert für hohe Frequenzen. Die Berechnung des Real- und Imaginärteils dieser Funktion ist ausführlich in [6] beschrieben. Es ergeben sich folgende Ausdrücke: 


$$
\begin{aligned}
& \chi^{\prime}(\omega)_{H N}=\chi_{\infty}+\left(\chi_{s}-\chi_{\infty}\right) \frac{\cos (\gamma \varphi)}{\left[1+2 \cdot\left(\omega \tau_{H N}\right)^{\alpha} \cdot \cos \left(\alpha \frac{\pi}{2}\right)+\left(\omega \tau_{H N}\right)^{2 \alpha}\right]^{\frac{\gamma}{2}}} \\
& \chi^{\prime \prime}(\omega)_{H N}=\left(\chi_{\infty}-\chi_{s}\right) \frac{\sin (\gamma \varphi)}{\left[1+2 \cdot\left(\omega \tau_{H N}\right)^{\alpha} \cdot \cos \left(\alpha \frac{\pi}{2}\right)+\left(\omega \tau_{H N}\right)^{2 \alpha}\right]^{\frac{\gamma}{2}}}
\end{aligned}
$$

mit

$$
\varphi=\arctan \left[\frac{\left(\omega \tau_{H N}\right)^{\alpha} \cdot \sin \left(\alpha \frac{\pi}{2}\right)}{1+\left(\omega \tau_{H N}\right)^{\alpha} \cdot \cos \left(\alpha \frac{\pi}{2}\right)}\right] .
$$

Der Parameter $\alpha$ kann zwischen eins und null variiert werden und führt zu einer Verbreiterung gegenüber einer Debye-Relaxation. Der Parameter $\gamma$ führt zu einem asymmetrischen Verlauf des Imaginärteils. AsymmetrieParameter kleiner als eins haben einen langsameren Abfall zu höheren Frequenzen $\chi^{\prime \prime} \propto f^{-\gamma}$ zur Folge.

Der Spezialfall $\gamma=1$ wird als Cole-Cole-Funktion bezeichnet, und der Fall $\alpha=1$ vereinfacht die HN-Funktion zu einer Form, die als Cole-Davidson Funktion bekannt ist [7].

Mit $\gamma=1$ und $\alpha=1$ vereinfachen sich die Gleichungen (2.20) und (2.21) wieder zu den Debye-Gleichungen.

\subsection{Wichtige Messmethoden}

In der Diskussion der Ergebnisse werden vergleichend vorherige Resultate verschiedener mechanischer Spektroskopiemethoden besprochen. Daher soll an dieser Stelle eine kurze Gegenüberstellung der wichtigsten Charakteristika gebräuchlicher Techniken erfolgen.

Dynamisch mechanische Analyse (DMA): Die dynamisch mechanische Analyse ist eine vielfach verwendete Technik, die in diesem Institut zur Untersuchungen an metallischen Proben und auch an Polymer-Proben genutzt wird [7, 48].

Messprinzip: An einer massiven Probe wird entweder gezogen oder auch gedrückt. Dazu wird ein Quarzstab verwendet, der die angelegte Kraft überträgt und dessen Position die aktuelle Auslenkung der Probe anzeigt. Sowohl die Erzeugung der Kraft als auch die Positionsdetektion erfolgen bei dem hier verwendeten Messaufbau „Perkin-Elmer, DMA 7" elektromagnetisch. Zusätzlich zu einer statischen Kraft kann eine dynamische Kraft im Frequenzbereich 
$1-50 \mathrm{~Hz}$ angelegt werden, um dynamische Relaxationsmessungen durchzuführen, wie in Abschnitt 2.1.1 beschrieben. Das Relaxationsspektrum kann durch Variation der Probentemperatur mit verschiedenen Heizraten im Bereich $70-900 \mathrm{~K}$ bei fest eingestellter Frequenz aufgenommen werden. Aus gemessener Schwingungsamplitude bei vorgegebener dynamischer Kraft kann der Elastizitätsmodul bestimmt werden. Die Phasenverschiebung zwischen Spannung und Dehnung kann ebenfalls direkt gemessen werden, wodurch der Verlustmodul berechnet werden kann. Details zu Messmodi und auch zur Datenanalyse sind z. B. in [49] gegeben.

Der Vorteil dieser Methode gegenüber anderen ist die große Flexibilität bezüglich Temperaturbereich, Heizrate und Frequenz. Auch Anregungsamplitude und statische Kraft können deutlich variiert werden. Nachteilig ist die Beschränkung auf massive Proben und die innere Reibung des Messaufbaus, der die Messung sehr kleiner Dämpfungen verhindert.

Ultraschall-Spektroskopie (USO): Die Ultraschall-Spektroskopie ist in den letzten Jahren in Zusammenarbeit mit Herrn Prof. Arnold in Göttingen etabliert worden. Die verwendete Anlage mit ihren Modifikationen ist in den Diplomarbeiten von A. Kahl [34] und T. Koeppe [50] genau beschrieben. Dabei wurden jeweils „Puls-Echo“- Messtechniken verwendet. Allgemeines zu Ultraschalltechniken kann in [51] nachgelesen werden.

Messprinzip: Durch einen hochfrequenten elektrischen Puls wird ein piezoelektrischer Schallwandler angeregt, worauf die erzeugte Ultraschall-Schwingung als Schallwelle durch eine massive Probe läuft. Die Probe wird zumeist durch einen kurzen Zylinder mit planparallel polierten Stirnflächen realisiert. An deren Enden tritt jeweils Reflexion der Welle auf. Die hin und her reflektierte Schallwelle tritt teilweise wieder in den Schallwandler, worauf dort ein elektrisches Signal proportional zur Schwingungsamplitude entsteht. Die Aufzeichnung dieses Signals als Funktion der Zeit führt zu einem „Echobild“ mit dem Laufzeiten $\Delta t$ in der Probe sehr genau bestimmt werden können. Bei genauer Kenntnis der Probenlänge $l$ und der Dichte $\rho$ kann aus der Laufzeit einer Scherwelle der Schermodul berechnet werden.

$$
G=\rho\left(\frac{l}{\Delta t}\right)^{2}
$$

Aus dem Abklingen der Echoamplituden kann außerdem Dämpfung in der Probe bestimmt werden. Für metallische Gläser bei tiefen Temperaturen erwies sich diese jedoch als zu klein um genau vermessen zu werden [50]. Verwendete Frequenzen liegen bei 5-20 MHz und der zugängliche Temperaturbereich von $\approx 4,2 \mathrm{~K}$ bis Raumtemperatur.

Der größte Vorteil dieser Technik ist die hohe relative Genauigkeit mit der auch kleine Relaxationseffekte studiert werden können, zudem ist man frei in 
der Wahl von transversaler oder longitudinaler Anregung. Nachteilig sind im Wesentlichen die Anforderung an relativ große, planparallel gefertigte Proben und die Einschränkung der Messtemperatur. Zudem schränken wenig präzise Längen und Dichtebestimmungen die absolute Messgenauigkeit ein.

Lokale mechanische Spektroskopie: Lokale mechanische Spektroskopie ist durch moderne Rastersondentechniken, insbesondere durch das Rasterkraftmikroskop (siehe Abschnitt 3.4.4) erst in letzter Zeit zugänglich geworden. Die einfachste Art ist die Aufnahme und Analyse von Kraft-AbstandsKurven [52]. Dabei wird die Messspitze lokal immer stärker auf die Probenoberfläche gedrückt. Die so erreichte Spitzenverbiegung ist auch von den lokalen elastischen Eigenschaften der Probe abhängig, was eine Kartierung dieser Eigenschaft möglich macht.

Eine weitere Möglichkeit, lokal elastische Eigenschaften auf einer Oberfläche zu messen, ist die Verwendung eines Atomic force acoustic microscopes. Die Technik und ihre Möglichkeiten sind detailliert beschrieben in [53] und [54]. Ein in diesem Institut aufgebautes Gerät wird auch in [55] vorgestellt und verwendet die Kontaktsteifigkeit von Spitze und Probe, die auch vom so genannten Indentationsmodul ${ }^{3} \mathrm{M}$ der Probenoberfläche abhängt. Auch quantitative Messungen des Indentationsmoduls sind hiermit möglich, jedoch nur bei fester Temperatur und nur sehr eingeschränkt frequenzabhängig.

Vibrating Reed: Die so genannte vibrating reed-Technik verwendet einen Biegebalken, der zu Eigenschwingungen, zumeist der ersten Biegemode, angeregt wird. Ein solcher Oszillator kann bei Verwendung von einkristallinem Silizium und im Vakuum eine Güte im Bereich $Q^{-1} \approx 10^{-5}$ bei Raumtemperatur aufweisen. Dies ermöglicht die Vermessung dünner Schichten. Dazu wird der Oszillator mit einer zu untersuchenden dünnen Schicht beschichtet und aus den Änderungen der Güte und der Eigenfrequenz des reeds auf die dünne Schicht zurück geschlossen. Diese Methodik ist mit der im Abschnitt 2.4 beschriebenen Doppelpaddel-Oszillator Technik eng verwandt. Die Schwingungsanregung kann elektrostatisch [43] oder auch über einen Plasmaplume [56] erfolgen. Die Detektion der Schwingungsamplitude wird typischerweise mit einem Laserstrahl als Lichtzeiger realisiert. Die Temperaturvariation erfolgt über Beheizung des Klemmblocks, der das reed aufnimmt. Eine Variation der Messfrequenz ist durch Änderung der Biegebalkengeometrie, insbesondere über die Dicke möglich. Dies geht allerdings zu Lasten von Empfindlichkeit oder Güte. Diese Methode bietet also bereits die Möglichkeit dünne Schichten temperaturabhängig zu vermessen. Da die Oszillatorverbiegung zu einer Streckung oder Stauchung der dünnen Schicht führt, wird überwiegend der Elastizitätsmodul gemessen. Zudem ist die Empfindlichkeit zumeist geringer als bei der Doppelpaddel-Oszillator Methode.

\footnotetext{
${ }^{3}$ Der Indentationsmodul $\mathrm{M}$ ist definiert durch: $M=\frac{E}{\left(1-\nu^{2}\right)}$, mit $\nu$ als Poissonzahl.
} 


\subsection{Der Doppelpaddel-Oszillator}

Dieser Abschnitt soll die Konzepte des Doppelpaddel-Oszillators methodisch erläutern. Die notwendigen experimentellen Vorgehensweisen folgen danach ab Kapitel 3.1.

Der Doppelpaddel-Oszillator (DPO) ist ein Oszillator, der sich durch seine spezielle Form besonders dazu eignet, dünne Schichten mechanisch zu vermessen. Das Design wurde 1985 von R. N. Kleinmann erstmals vorgestellt und bereits darauf hingewiesen, dass die sehr hohe Güte die mechanische Analyse von dünnen Schichten ermöglicht [57]. Eine auf den Oszillator aufgebrachte dünne Schicht ändert dabei dessen Eigenschaften messbar. Das prinzipielle Design ist in der Abbildung 2.3 schematisch abgebildet und auch, zur Demonstration der makroskopischen Abmaße, fotografisch auf Millimeterpapier auf Seite 46.

\subsubsection{Eigenmoden}

Aufgrund der komplizierten Geometrie des DPOs existieren eine Vielzahl von Eigenmoden, die angeregt werden können. Durch Vergleich von FiniteElemente Rechnungen mit gemessenen Frequenzspektren [58] und auch durch Laser-Doppler-Vibrometry konnten die niederfrequenten Eigenmoden identifiziert und für einkristallines Silizium mit 0,3 mm Dicke berechnet werden. Die Abbildung 2.3 zeigt einige dieser Moden schematisch. Die Kurzbezeichnung der Moden ist in Tabelle 2.1 übersetzt.

\begin{tabular}{|c|c|c|c|}
\hline Kurzform & Englische Bez. & Deutsche Bez. & Freq. [Hz] \\
\hline CL1 & 1st cantilever & Erste Biegemode & 280 \\
\hline ST & symmetric torsion & Symmetrische Torsion & 441 \\
\hline WW1 & 1st windshield wiper & Erste Scheibenwischer & 889 \\
\hline CL2 & 2nd cantilever & Zweite Biegemode & 1703 \\
\hline CL3 & 3rd cantilever & Dritte Biegemode & 3340 \\
\hline AS1 & 1st antisymm. tor. & Erste antisymm. Torsion & 4728 \\
\hline FL1 & 1st flapping & Erste Umschlagmode & 5295 \\
\hline AS2 & 2nd antisymm. tor. & Zweite antisymm. Torsion & 5515 \\
\hline
\end{tabular}

Tabelle 2.1: Wichtige Eigenmoden eines DPOs, graphisch dargestellt in Abb. 2.3, Eigenfrequenzen entnommen aus [58].

Bedingt durch die Art von Anregung und Detektion bei dem hier verwendeten Messaufbau werden die WW1 und höhere ,Scheibenwischer"-Moden weder angeregt noch gemessen. Auch Umschlagmoden, wie die FL1, treten in der Praxis nicht auf. 

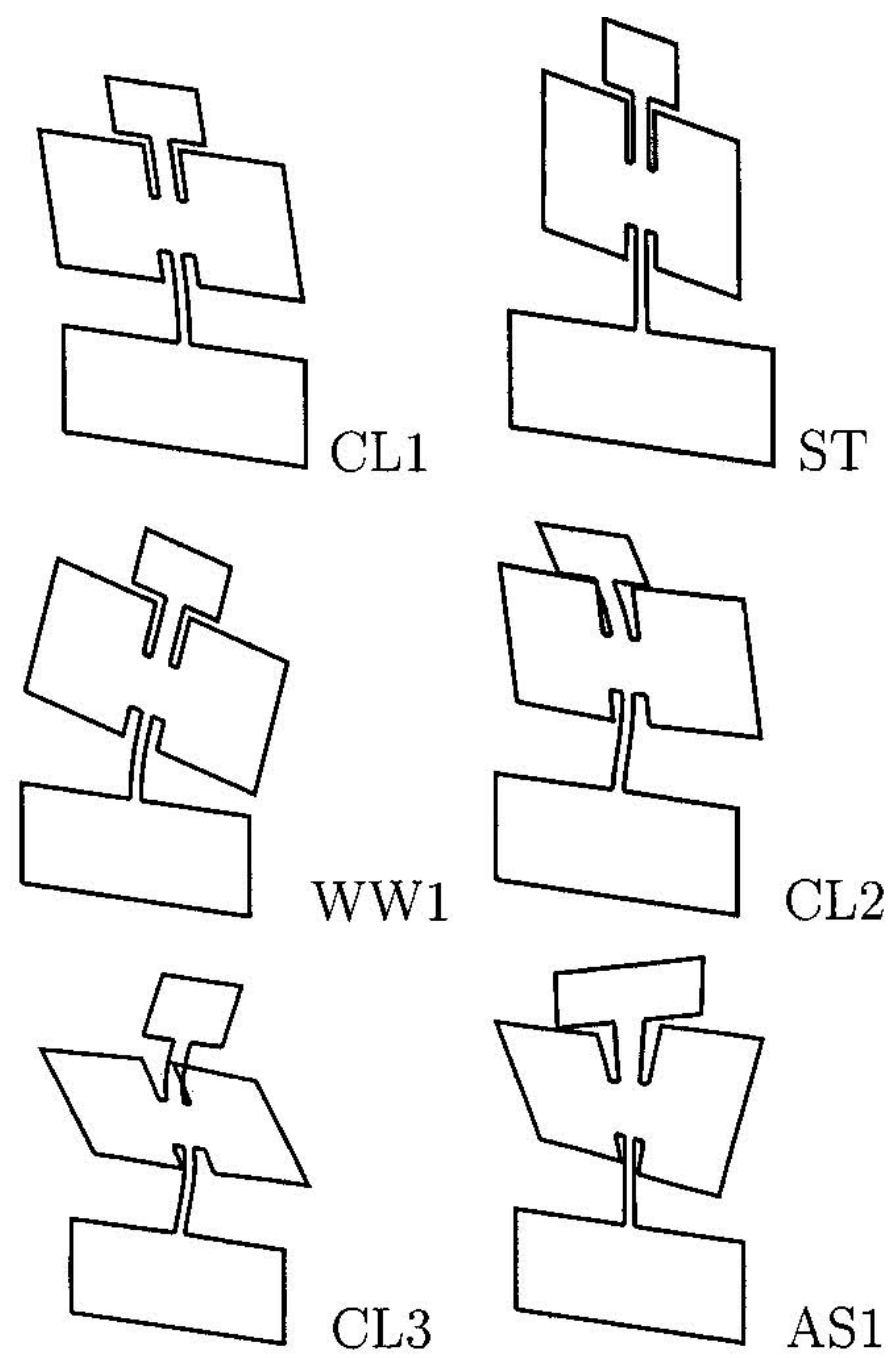

WW1
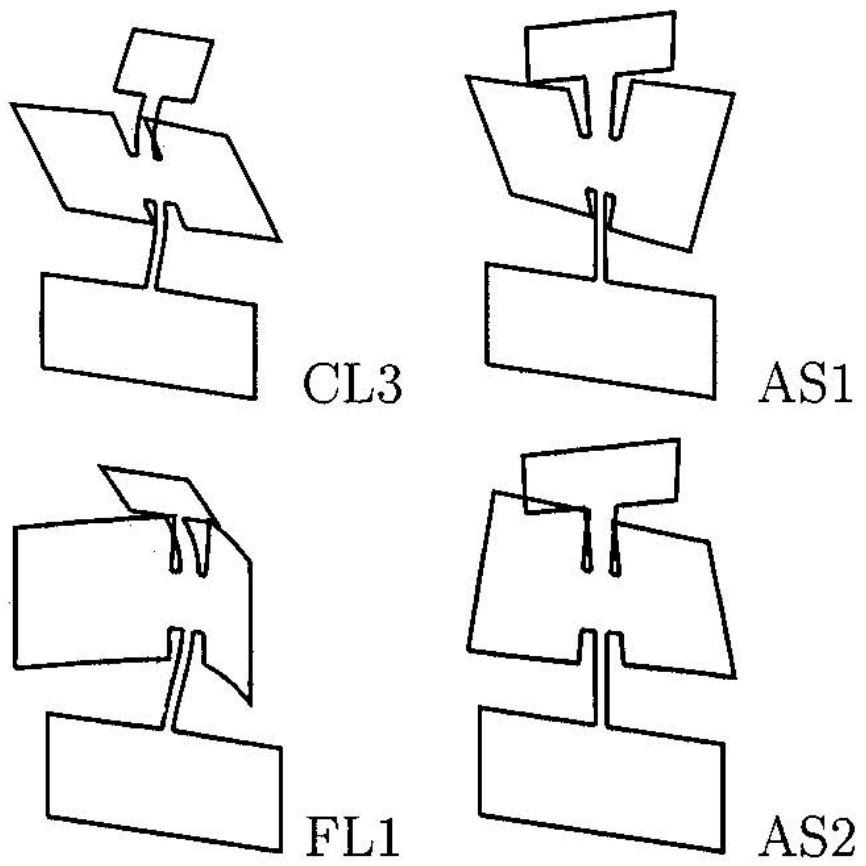

Abbildung 2.3: Schematische Darstellung einiger Moden des DoppelpaddelOszillators, Zeichnung aus [58]. 
Ein mit dem neuen Messaufbau gemessenes Frequenzspektrum zeigt Abbildung 2.4. Man erkennt, dass nahezu alle in Tabelle 2.1 angegebenen Moden auch angeregt werden können. Die relative Höhe der Amplituden sagt nichts über die Anregbarkeit der Moden aus, da die Halbwertsbreiten der Moden in der Frequenz noch wesentlich schmaler sind als die hier gewählte Schrittweite. Die Indizierung der Ausschläge erfolgt durch Vergleich mit den bereits bekannten Frequenzen der einzelnen Moden. Weitere nicht indizierte Signale sind überwiegend durch akustische Beeinflussung der Messung zu erklären.

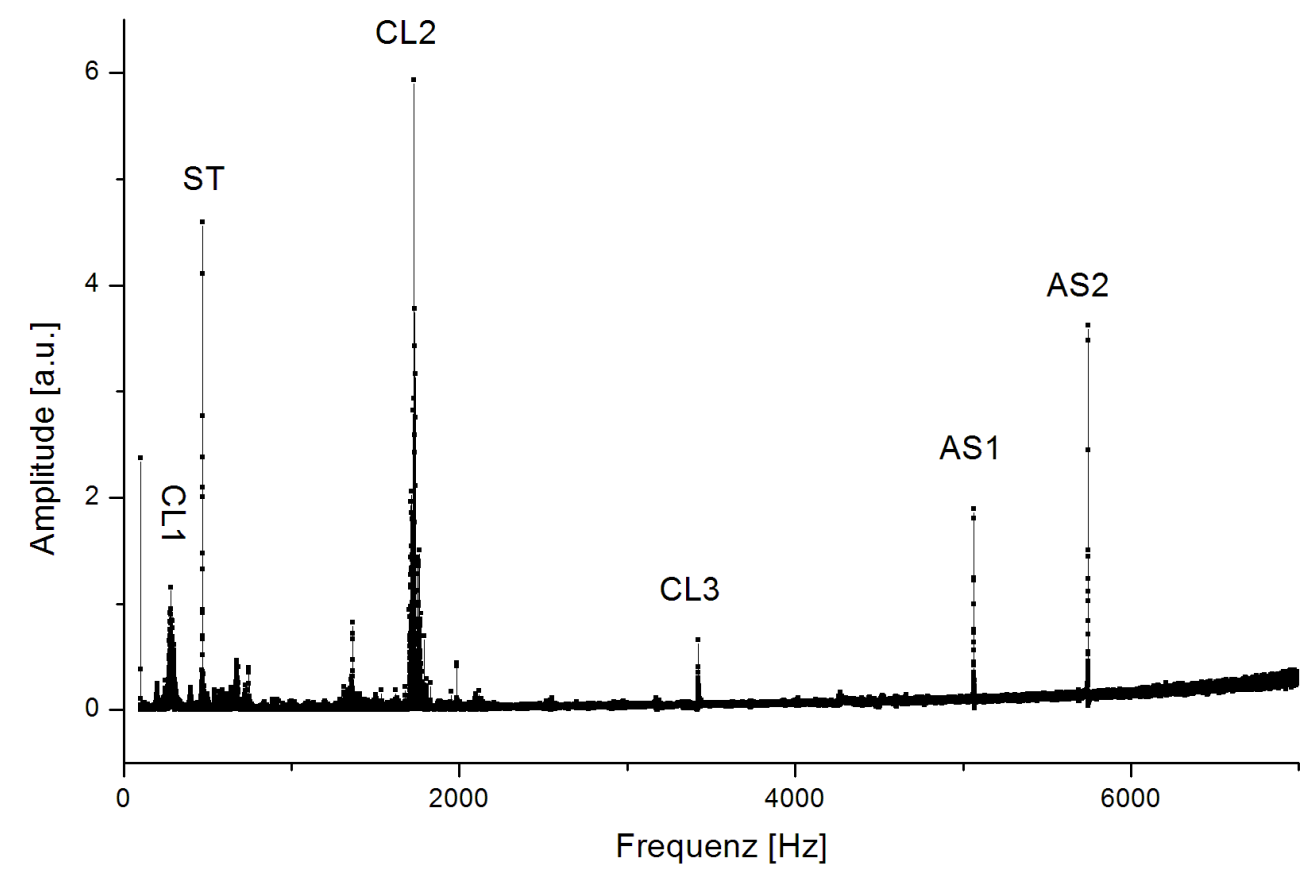

Abbildung 2.4: Ein Frequenzscan von DPO_DB_02 zeigt das typische DPO Spektrum mit den bekannten Moden.

Im Folgenden wird fast nur noch die AS2-Mode behandelt. Diese Mode bietet gegenüber den anderen Moden eine Reihe von Eigenschaften, die alle für diese Studie von Vorteil sind:

- Der Hauptgrund für die Verwendung dieser Mode ist die sehr geringe Eigendämpfung, die der Oszillator darin aufweist. Dies erklärt sich in der besonderen Schwingungsgeometrie, bei der sich der obere Teil, auch als Kopf bezeichnet, gegen den mittleren Teil verdreht. Dieser Mittelteil kompensiert das Drehmoment durch Querverdrehung der Seitenteile („Flügel“) im Gegentakt. Deshalb wird idealerweise keine Energie 
über den „Fuß`, also an der Klemmung dissipiert. Diese Annahme kann durch die Laser-Doppler-Vibrometrie Messungen unterstützt werden. Außerdem konnte eine Güte von $Q^{-1} \approx 2 * 10^{-8}$ unterhalb von $10 \mathrm{~K}$ gemessen werden [58].

- In [58] wurde u. a. die Reproduzierbarkeit der Dämpfung beim zyklischen Heizen und Kühlen untersucht. Dabei erwies sich die gemessene Dämpfung der AS2-Mode als besonders stabil.

- Zuletzt ist es von Vorteil, dass ein aufgebrachter, fest haftender Film bei der AS2-Mode des Paddels im Wesentlichen nur geschert wird, da die größte Auslenkung an dem „Hals“ in Form einer Verdrehung auftritt. Der Schermodul ist aber am empfindlichsten beim Übergang vom Festkörper zu einer Flüssigkeit, da diese im Bornschen Grenzfall überhaupt keinen Schermodul mehr besitzt. Damit eignet sich der Schermodul in besonderer Weise, um Glasübergänge zu studieren.

\subsubsection{Bestimmung der Dämpfung}

Die Dämpfung des Oszillators bei einer bestimmten Mode kann direkt durch Vermessung der Frequenzbreite der Resonanzkurve bestimmt werden. Dabei ist der inverse Qualitätsfaktor $Q^{-1}$ definiert als:

$$
Q^{-1}=\frac{\omega_{2}-\omega_{1}}{\omega_{r}}
$$

Der Ausruck $\omega_{2}-\omega_{1}$ beschreibt die Halbwertsbreite und $\omega_{r}$ das Maximum der Resonanzkurve.

Bei kleinen Dämpfungen und kleinen Änderungen in Q ist diese Methode jedoch nicht genau genug, zudem muss die Resonanzkurve sehr langsam durchfahren werden, um im eingeschwungenen System zu messen. Daher wird in dieser Arbeit die Dämpfung ausschließlich durch „Ring-downs" gemessen: Das zuvor resonant angeregte Paddel schwingt frei aus, man beobachtet das Abklingen der Schwingungsamplitude $A$ als Funktion der Zeit $t$. Für einen gedämpften harmonischen Oszillator ist diese gegeben durch:

$$
A(t)=A_{0} \exp \left(-\pi f Q^{-1} \cdot t\right)
$$

Ein typischer gemessener Amplitudenverlauf ist in Abbildung 2.5 dargestellt. Man erkennt in der logarithmischen Auftragung einen streng exponentiellen Abfall.

Der inverse Qualitätsfaktor wird bestimmt, indem man aus einer Auftragung $\ln A(T)$ gegen die Zeit das logarithmische Dekrement $\Lambda=\pi f Q^{-1}$ ermittelt und dieses anschließend durch gemessene Frequenz $f$ und $\pi$ teilt. 


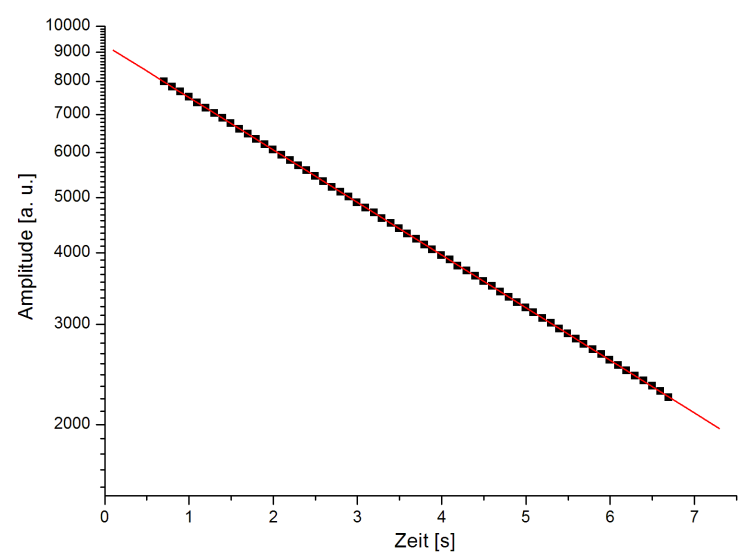

Abbildung 2.5: Ein typischer Amplitudenverlauf beim „Ring-down“. In der logarithmischen Darstellung mit linearem Fit beobachtet man einen rein exponentiellen Verlauf. Die ersten 0,7s werden nicht aufgezeichnet, da noch elektrische Störungen durch das Ausschalten des Antriebs auftreten können. Die Aufzeichnung endet bei 1/4 der Startamplitude.

Dieser Teil der Auswertung wird durch das Messprogramm, geschrieben in LabView, direkt durchgeführt, wobei zur Kontrolle der Anpassungsfehler bei der Bestimmung von $\Lambda$ protokolliert wird. Für eine spätere Kontrolle wird jeder gemessene Ring-down auch in Rohform aufgezeichnet.

\section{Amplitudenabhängigkeit:}

Um so reproduzierbar wie möglich zu messen, finden die Ring-downs stets in einem sehr engen Amplitudenbereich statt. Die Amplitudenabhängigkeit der Dämpfung wurde für verschiedene Paddel getestet und erwies sich (in fast allen Fällen) als sehr klein. Nur die sehr hohe Messgenauigkeit der DPOMessungen erlaubt es, kleine relative Änderungen in der Dämpfung $(\leq 1 \%)$ die sich etwa bei einer Verdopplung der Amplitude ergeben, noch zu detektieren. (Lediglich bei einem vermessenem Paddel erwies sich die Dämpfung deutlich Amplituden-abhängig. In diesem Fall kann auch ein geometrischer Effekt der elektrostatischen Anregung ausschlaggebend gewesen sein.)

\subsubsection{Einfluss des Films auf das DPO}

Die mathematische Behandlung des mechanischen Systems Schicht-DPO ist bereits in [6] genau ausgeführt. An dieser Stelle sollen nur die wesentlichen Abhängigkeiten dargestellt werden.

Frequenzverschiebung: Bringt man einen dünnen Film auf das DPO auf, so ändert sich im Allgemeinen die Frequenzlage bei gleicher Temperatur. 
Bereits die lineare Antwort des Systems, gezeigt in Abbildung 2.5, rechtfertigt die Annahme, dass dieser Oszillator mit einem harmonischen Oszillator verglichen werden kann. Für diesen ist die Eigenfrequenz $\omega_{0}$ gegeben durch:

$$
\omega_{0}=\sqrt{\frac{k}{m}}
$$

Die Federkonstante $k$ entspricht hierbei der Biege- bzw. Schersteifigkeit des DPOs und die Masse $m$ dem Trägheitsmoment bezüglich der angeregten Mode. Beide Größen werden durch den aufgebrachten Film modifiziert. Zum einen bringt die zusätzlich aufgebrachte Masse eine größere Trägheit und damit eine Frequenzabsenkung mit sich, zum anderen liefert auch der gedehnte oder gescherte Film einen rücktreibenden Kraftbeitrag bei der Schwingung des DPOs.

Die mathematische Behandlung einer AS2-Mode, beinhaltend einer Näherung für kleiner Änderungen und für dünne Schichten, liefert ${ }^{4}$ :

$$
\frac{\Delta f_{A S 2}}{f_{A S 2}}=\frac{3 G_{F i l m} d_{F i l m}}{2 G_{S i} d_{S i}}-\frac{\rho_{F i l m} d_{F i l m}}{2 \rho_{S i} d_{S i}}
$$

Aus erstem Term folgt, dass die Frequenzverschiebung $\Delta f$ direkt proportional zur Dicke des Films $d_{\text {Film }}$ und dessen Schermodul $G_{\text {Film }}$ ist, wie bereits zuvor angedeutet. Der zweite Term beschreibt, wegen des Vorzeichens, eine massenbedingte Absenkung der Resonanzfrequenz, in diesem Fall durch das Produkt von Filmdicke und Filmdichte $\rho_{\text {Film }}$.

Um aus der gemessenen Resonanzfrequenz $f_{A S 2}$ und ihrer Verschiebung den gesuchten Schermodul $G_{\text {Film }}$ des Films zu berechnen, müssen die anderen Parameter bekannt sein. Schermodul ${ }^{5}(66,2 \mathrm{GPa})$ und Dichte $\left(2,33 \mathrm{~g} / \mathrm{cm}^{3}\right)$ von Silizium sind aus der Literatur bekannt [59]. Die Dicke des DPOs $d_{S i}$ ist ebenfalls gut bekannt, es verbleiben noch die Bestimmung der Filmdichte $\rho_{\text {Film }}$ und die genaue Schichtdicke $d_{\text {Film }}$.

Die Filmdichte wird als arithmetisches Mittel der Dichten der Legierungskomponenten unter Berücksichtigung ihres atomaren Anteils $w_{i}$ berechnet $^{6}$ :

$$
\rho_{\text {Film }}=\sum w_{i} \rho_{i}
$$

Die genauen atomaren Konzentrationen der Elemente in den Filmen werden durch EDX-Messungen ermittelt. Die Schichtdicken werden ebenfalls durch

\footnotetext{
${ }^{4}$ Der Index $S i$ steht für das leere Doppelpaddel, da dieses fast ausschließlich aus Silizium gefertigt wird.

${ }^{5}$ Die Symmetrie-, Drehachse des Paddels liegt in der Si-〈110 $\rangle$-Kristallrichtung.

${ }^{6}$ Eine solche Berechnung vernachlässigt das excess-Volumen [60] der eutektischen Legierung und Oberflächeneffekte.
} 
separate Messungen mittels Kleinwinkel-Röntgenstreuung bestimmt. Beide Verfahren werden in den Abschnitten 3.4.1 und 3.4.3 kurz beschrieben.

\section{Dämpfungseinfluss:}

Eine besondere Stärke der Doppelpaddel-Oszillatoren ist die besondere Empfindlichkeit im Bezug auf ihre Eigendämpfung. Auch sehr dünne Filme führen zu messbaren Änderungen in der Dämpfung, womit auf den Film zurück geschlossen werden kann. Der elastische Film führt zwar zu einer Erhöhung der bei einer Schwingung gespeicherten Energie $W$, jedoch tritt dort auch ein zusätzlicher Energieverlust $\Delta W$ durch innere Reibung im Film auf.

Der Qualitätsfaktor, definiert wie in Gleichung (2.11), kann für ein DPO mit Film berechnet werden:

$$
2 \pi Q_{D P O}^{-1}=\frac{\Delta W_{\text {Substrat }}+\Delta W_{\text {Film }}}{W_{\text {Substrat }}+W_{\text {Film }}}
$$

Da für dünne Filme $W_{\text {Film }}$ gegenüber $W_{\text {Substrat }}$ vernachlässigt werden kann, führt nach dieser Gleichung ein aufgebrachter Film immer zu einer erhöhten Dämpfung. Gleichung (2.29) kann vergleichbar mit der Rechnung zur Frequenzverschiebung umgeformt werden zu:

$$
\Delta Q^{-1}=Q_{D P O}^{-1}-Q_{\text {Substrat }}^{-1}=\frac{3 G_{\text {Film }} d_{\text {Film }}}{G_{\text {Substrat }} d_{\text {Substrat }}} \cdot Q_{\text {Film }}^{-1}
$$

Die experimentell direkt zugängliche Änderung des inversen Qualitätsfaktors des DPOs ist damit proportional zur Dämpfung in der Schicht.

\section{Komplexer Schermodul der Schicht}

Durch Messung sowohl von Frequenzverschiebung als auch Dämpfungsanstieg, hervorgerufen durch eine Schicht, kann der komplexe Schermodul der dünnen Schicht selbst berechnet werden. Dazu werden zunächst $G_{\text {Film }}$ und $Q_{\text {Film }}^{-1}$ berechnet. Nun verwendet man die Gleichungen (2.9) und (2.10) für den komplexen Schermodul und erhält:

$$
G_{F i l m}^{\prime}=\frac{G_{F i l m}}{\sqrt{1+\left(Q_{F i l m}^{-1}\right)^{2}}}
$$

und:

$$
G_{\text {Film }}^{\prime \prime}=\frac{Q_{\text {Film }}^{-1} G_{\text {Film }}}{\sqrt{1+\left(Q_{\text {Film }}^{-1}\right)^{2}}}
$$




\subsubsection{Reproduzierbarkeit und Messempfindlichkeit}

In dieser Arbeit wird die sehr sensitive DPO Messmethode an die Grenze des experimentell Machbaren geführt. Dabei sollen die gewonnenen Ergebnisse aber stets belastbar und unstreitig signifikant bleiben. In diesem Abschnitt werden Abschätzungen und experimentelle Befunde bezüglich der Messgenauigkeit den eigentlichen Ergebnissen vorweg gestellt. Auch bei der Vorstellung der im nächsten Abschnitt beschriebenen experimentellen Techniken wird auf diesen wichtigen Punkt noch mehrfach hingewiesen.

Um sich die Empfindlichkeit des Doppelpaddel-Oszillators bildlich vor Augen zu führen, betrachtet man die Relation von Filmvolumen zu Paddelvolumen. Ein relativ dicker Film von z. B. 300 nm Dicke ändert die Eigenschaften von einem massiven Paddel, dessen Dicke typischerweise 1000 Mal größer ist. Ein amorpher Film lässt, pro Volumen, eine höhere Dämpfung erwarten als das einkristalline Silizium, aus dem der Oszillator gefertigt ist, dennoch sollen hier noch wesentlich dünnere Filme nicht nur ,festgestellt" sondern auch bezüglich ihrer temperaturabhängigen Dämpfung vermessen werden.

Die Dämpfungsmessung erfolgt, wie bereits in Absatz 2.4.2 beschrieben, über Ring-downs. Ein kurzes Beispiel zu den Messgenauigkeiten der DPOMethode zeigt deutlich, dass hier die Reproduzierbarkeit den beschränkenden Faktor darstellt: Der Anpassungsfehler des logarithmischen Dekrements $\Lambda=0,16712 \mathrm{~s}^{-1}$ beträgt in diesem Fall $7,8 * 10^{-5} \mathrm{~s}^{-1}$. Nimmt man die zugehörige Frequenzmessung als fehlerlos an, so folgt aus der Fehlerfortpflanzung für $Q^{-1}: Q^{-1}=1,1551 * 10^{-5} \pm 5 * 10^{-9}$. Dies entspricht einer relativen Genauigkeit von 0,05\%. Die Messung eines leeren Paddels zeigt jedoch eine viel höhere Streuung der Messwerte. Passt man den Dämpfungsverlauf mit einem Polynom z. B. 3. Ordnung an, so ergibt sich eine Standardabweichung der Messwerte von diesem Polynom von typischerweise $1 * 10^{-8}$ bis $1 * 10^{-7}$, je nach Paddel und Temperaturbereich. Für die Bestimmung der Differenzdämpfung $\Delta Q^{-1}(T)$ wird ein solches Polynom als Leermessung zugrunde gelegt. Die Reproduzierbarkeit dieses Verlaufs wird mit dem im Abschnitt 3.1.2 beschriebenen Messablauf sichergestellt. Es können mehrere Messdurchläufe des leeren DPOs mit einem Polynom angepasst werden, gezeigt in Abbildung 4.9 auf Seite 65. Aufgrund der hohen Messpunktedichte, aus denen sich das Polynom berechnet, wird der Fehler der Leermessung anschließend vernachlässigt. Es verbleibt die Messungenauigkeit der einzelnen Messung eines Paddels mit Schicht, der mittlerer Wert der oben angegeben Streuung, $5 * 10^{-8}$. Eine amorphe $\mathrm{PdCuSi}$-Schicht mit $200 \mathrm{~nm}$ Dicke führt zu einer maximalen zusätzlichen Dämpfung von $\Delta Q^{-1} \approx 6 * 10^{-6}$. In linearer Extrapolation der Dämpfung mit der Schichtdicke ist ein Film von $2 \mathrm{~nm}$ Dicke also gerade noch zu detektieren. 
Es muss bei der Angabe der Messungenauigkeit angefügt werden, dass es Fehlerquellen wie Oberflächenkontaminationen, nicht stabiler Sitz des Paddels in der Halterung und Temperaturabweichungen gibt, die den angegeben Messfehler deutlich übersteigen können. Das nachfolgende Kapitel 3 gibt detailliert Auskunft über Techniken zur Vermeidung bzw. Kontrolle dieser Fehlerquellen.

Die Frequenzmessung zur Bestimmung der Frequenzabweichung $\Delta f$ erfolgt mit einem Frequenzzähler, während das Paddel in einem quasi-PLL bei der Resonanzfrequenz schwingt. Der Unterschied von Resonanz- zu Eigenfrequenz kann wegen der sehr geringen Dämpfung des Paddels vernachlässigt werden. Die Paddeltemperatur ändert sich bei der Verwendung von Temperaturrampen dauernd, jedoch erfolgt die Messung von Frequenz und Temperatur automatisiert und nur wenige ms verzögert. Da Frequenzen i. A. sehr genau gemessen werden können, stellt sich in direkter Analogie zum Fehler der Dämpfungsmessung eher die Frage nach der Reproduzierbarkeit. Die Streuung der Frequenzmesswerte als Funktion der Temperatur um ein ausgleichendes Polynom 2. Ordnung liegt in der Größenordnung von 0,05 Hz. Für eine Schwingung im kHz-Bereich eines geheizten Oszillators ist das bereits eine bemerkenswerte Stabilität. Zwei aufeinander folgende Messdurchläufe unterscheiden sich um weniger als $0,1 \mathrm{~Hz}$ beim Heizen, was den statistischen Fehler bei der Bestimmung von $\Delta f(T)$ in etwa abschätzt. Mit dieser Genauigkeit können PdCuSi-Schichten ab 3-4 nm gemessen werden. Bei der Frequenzmessung bei konstanten $40^{\circ} \mathrm{C}$ ist die Genauigkeit etwa 10 Mal besser.

Die Gleichung (2.27) für die Frequenzverschiebung liefert unter der Annahme $G_{\text {Film }}=0$ eine maximale Frequenzabsenkung durch den Film bei bekannter Dichte und Dicke. Die Auswertung dünner Filme bei hohen Temperaturen weist jedoch teilweise größere Verschiebungen als erwartet auf, wodurch die Auswertung unphysikalische negative Werte für $G_{\text {Film }}$ liefert. Dieser Effekt ist bereits in [6] beobachtet worden, dort wurde u. a. die Gültigkeit der Gleichung (2.27) angezweifelt. Zu Beginn dieser Arbeit ergab eine Testmessung einer reinen Palladiumschicht nahe Raumtemperatur das korrekte Schermodul von 45 GPa (Literaturwert: 44 GPa [61]), was die Gültigkeit dieser Gleichung unterstützt. Daher kann die zu große Verschiebung nur durch kleine Temperaturverschiebungen von DPO mit Film zu DPO ohne Film erklärt werden. Quantitative Aussagen über die Schersteifigkeit der sehr dünnen Filme sind damit bei hohen Temperaturen nur bedingt möglich. Diese Einschränkung gilt nicht für die Dämpfungsbestimmung, da in diese nur die sehr genaue absolute Frequenz eingeht. 


\section{Kapitel 3}

\section{Experimentelles}

In diesem Kapitel werden die für das experimentelle Arbeiten notwendigen Techniken beschrieben. Dabei liegt der Schwerpunkt auf der genauen Beschreibung der Doppelpaddel-Oszillator Messungen, da diese nicht standardisiert sind und in dieser Arbeit im Vordergrund stehen.

\subsection{Der DPO-Messaufbau}

Für die DPO-Messungen wurde zu Beginn der Arbeit ein völlig neuer Messaufbau konzipiert und gebaut. Die Grundkonzeption für den UHV-Hochtemperatur-Messeinsatz wurde dabei von Dr. Peter Rösner übernommen, der zuvor grundlegende Experimente zum wing in metallischen Gläsern an derselben Anlage durchgeführt hat [6].

Der mechanische Aufbau ist an einem CF-250 Blindflansch mittels Edelstahlstäben aufgehängt, um alle Messungen und Beschichtungsvorgänge unter UHV-Bedingungen durchführen zu können und um den Messaufbau weitestgehend von äußeren Einflüssen wie Lärm, Vibrationen und Temperaturschwankungen zu entkoppeln. Der CF-Flansch wurde so gebaut, dass sämtliche für Messung und Beschichtung notwendigen Durchführungen vorhanden sind: Ein Beobachtungsfenster, zwei Koaxialdurchführungen (DPOAnregung und Messung), eine Stromdurchführung zur Beheizung, zwei Thermoelementleitungen Typ K, eine Durchführung für flüssigen Stickstoff und eine Drehdurchführung für den Shutter.

Eine schematische Darstellung, erstellt mittels Auto Desk Inventor, des neuen Einsatzes zeigt Abbildung 3.1 auf Seite 34.

Da sich die Details teilweise deutlich gegenüber dem Aufbau in [12] bzw. [6] unterscheiden werden die Bauelemente hier kurz vorgestellt: 


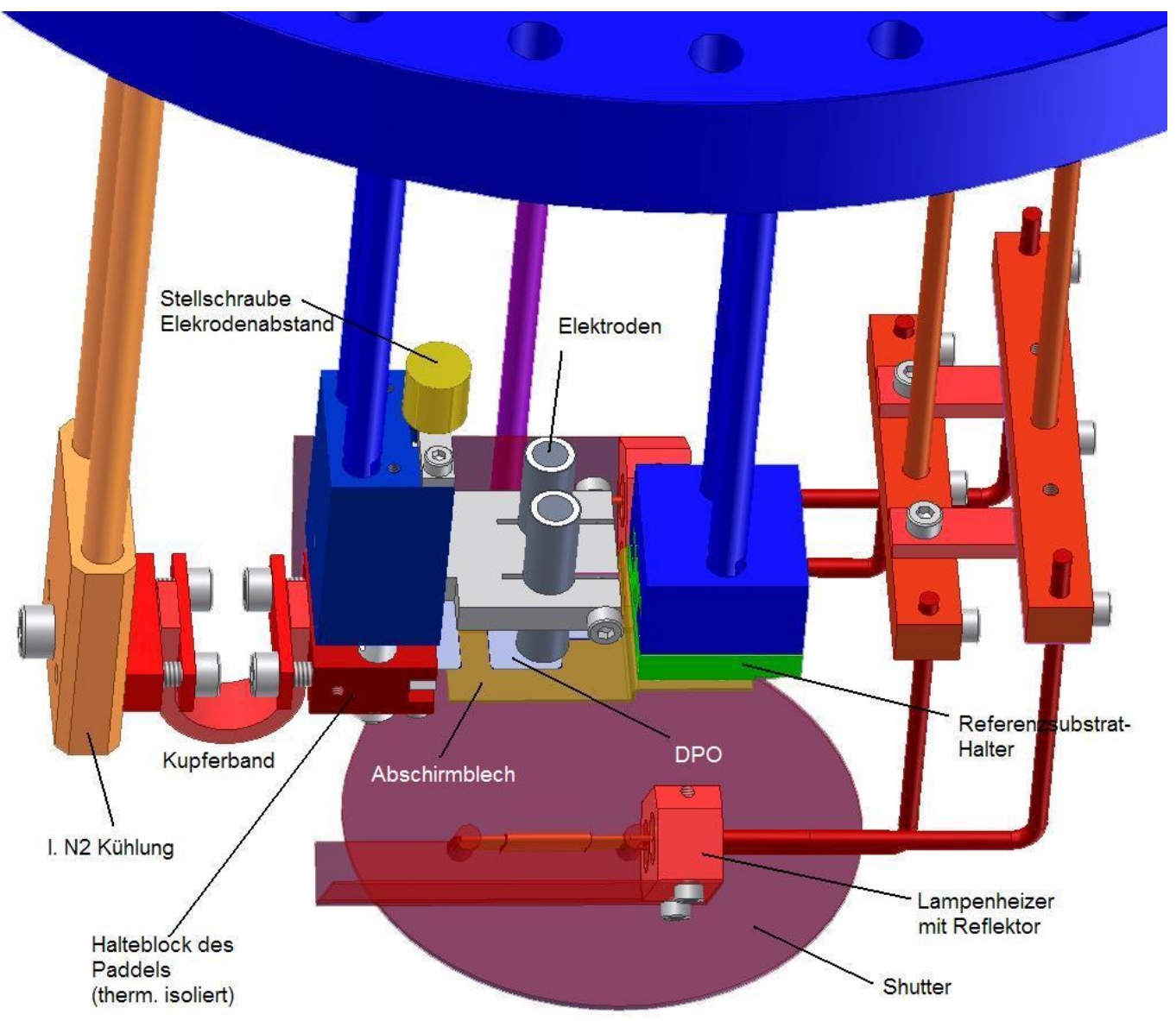

Abbildung 3.1: Der UHV Einsatz „DPO Evolution“. 
- Klemmblock: Der Klemmblock wird verwendet um das Paddel fest und definiert aufzunehmen. Der eigentlichen Klemmung kommt dabei besonderer Beachtung zugute, da sie die mechanischen Eigenschaften des Paddels maßgeblich beeinflussen kann. Eine Erörterung darüber findet sich z. B. in [58].

Die Klemmung wird hier, wie bei einem Schraubstock, durch eine feste und eine mittels Schraubgewinde vorgetriebene Klemmbacke realisiert. Die unterschiedlichen thermischen Ausdehnungen des Silizium-Paddels und der metallischen Klemmung erweisen sich bei großen Temperaturintervallen während der Messungen als kritisch. Um dieses zu kompensieren wird außer dem Paddelfuß stets ein ca. 0,6 mm dickes Stück Silizium mit in den Klemmfuß eingeklemmt, welches wie eine Feder eine gewisse „Vorspannung“ erlaubt und Spannungen auffangen kann.

Die Temperaturmessung erfolgt ebenfalls an dem Klemmblock mittels eines angeklemmten Thermoelementes. Die Position kann in der Abbildung 3.2 links auf der Frontseite des Klemmblockes erkannt werden. Um einen raschen Temperaturausgleich über den gesamten Block zu erreichen wurde dieser aus einem massiven Stück Silber gefräst. Die Bestimmung der Paddelkopf-Temperatur über die Blocktemperatur wird später im Kapitel 3.1.1 erläutert.

- $\mathbf{N}_{\mathbf{2}}$ - Kühlung: Um den messbaren Temperaturbereich zu erweitern wurde eine Kühlmöglichkeit mittels flüssigen Stickstoffs geschaffen. Der Stickstoff fließt durch den Kühlblock hindurch und kühlt diesen sehr effizient ab. Selbiger ist mit dem Klemmblock über ein dickes Band aus OFHC- (oxygen free high conductive) Kupfer verbunden. (Abbildung 3.2). Der Klemmblock wiederum ist gegen den übrigen Aufbau soweit möglich isoliert. Dazu kommen drei MACOR ${ }^{\circledR}$-Hohlröhrchen zum Einsatz. Im Vakuum kühlt dieser Aufbau den Block und damit das eingespannte Paddel effektiv ab. Abhängig von den genauen Abständen im fertig montierten Zustand kann eine Temperatur von $\approx-160{ }^{\circ} \mathrm{C}$ in $2 \mathrm{~h}$ erreicht werden.

- Elektroden: Die beiden Elektroden für die kapazitive Anregung und Detektion der Oszillator-Schwingungen sind ein Eigenbau, der in gleicher Weise wie in [6] beschrieben, hergestellt wurde. Der Abstand zwischen Elektrodenunterseite und Paddeloberseite kann mit einer Stellschraube, deren Gewinde eine geringe Steigung aufweist, sehr präzise eingestellt werden. Praktikable Abstände liegen etwas unter $1 \mathrm{~mm}$ und müssen nach einem Paddelneueinbau jeweils optimiert werden. Zu geringe Abstände beeinflussen dabei die Eigenschaften des Paddels, wäh- 
rend zu große Distanzen sowohl die Anregung als auch die Messbarkeit einer Schwingung stark verringern.

- Lampenheizung: Das Paddel wird über das Strahlungsfeld zweier Filmlampen (900W, Halogen Photo Optic Lamp) beheizt. Durch den Einsatz von Reflektoren ist es mit diesem Einsatz möglich, das Paddel auf über $450{ }^{\circ} \mathrm{C}$ zu heizen, ohne einen Strahlungsschirm um den Einsatz herum zu verwenden. Der fehlende Strahlungsschirm erleichtert die Temperaturregelung, da die Verlustleistung des Einsatzes höher ist, erfordert aber unbedingt eine Wasserkühlung der UHV-Anlagenwände und aller empfindlichen Bauteile. Die notwendige elektrische Leistung von bis zu 400 Watt liefert eine leistungsfähige Gleichstrom-Quelle.

- Referenzsubstrathalter: Es können zur Kontrolle der Aufdampfparameter und zu Referenzzwecken Substrate über die Schleusenkammer der UHV-Beschichtungsanlage ein- und ausgeschleust werden. Das geschlossene Shutter verdeckt sowohl das Doppelpaddel als auch das Referenzsubstrat. Um das Referenzsubstrat zu bedampfen, ohne das Paddel beschichten zu müssen, kann das in Abbildung 3.1 gelb dargestellte Abschirmblech verwendet werden.

- Shutter: Der Shutter wird manuell an der Drehdurchführung bedient.

\subsubsection{Temperatursteuerung}

Die Beheizung des Paddels erfolgt, wie oben beschrieben, über Strahlungslampen. Dabei befindet sich der Hals des Paddels im Zentrum des Strahlungsfeldes. Der Klemmblock, an dem die Temperatur während der Messungen erfasst wird, erfährt nur noch eine geringe Beheizung über die Lampen. Der Paddelkopf entzieht sich einer direkten Temperaturmessung dadurch, dass ein angebrachtes Thermometer die Schwingungen behindern würde, und eine Messung mittels Strahlungsthermometer ist durch die Nähe der viel heißeren Glühlampen unpraktikabel. Die Temperaturen von Paddelkopf und Klemmblock sind wegen des direkten thermischen Kontaktes jedoch gut aneinander gekoppelt. Über ein zweites sehr kompaktes Thermoelement, welches auf den Paddelkopf mittels Leitsilber aufgeklebt wird, kann die Paddelkopftemperatur als Funktion der Blocktemperatur bestimmt werden ${ }^{1}$. Damit können die gewonnenen Messdaten nach Abschluss der mechanischen Messungen der

\footnotetext{
${ }^{1}$ Das Paddel kann mit diesem Anbauteil nicht mehr schwingen.
} 


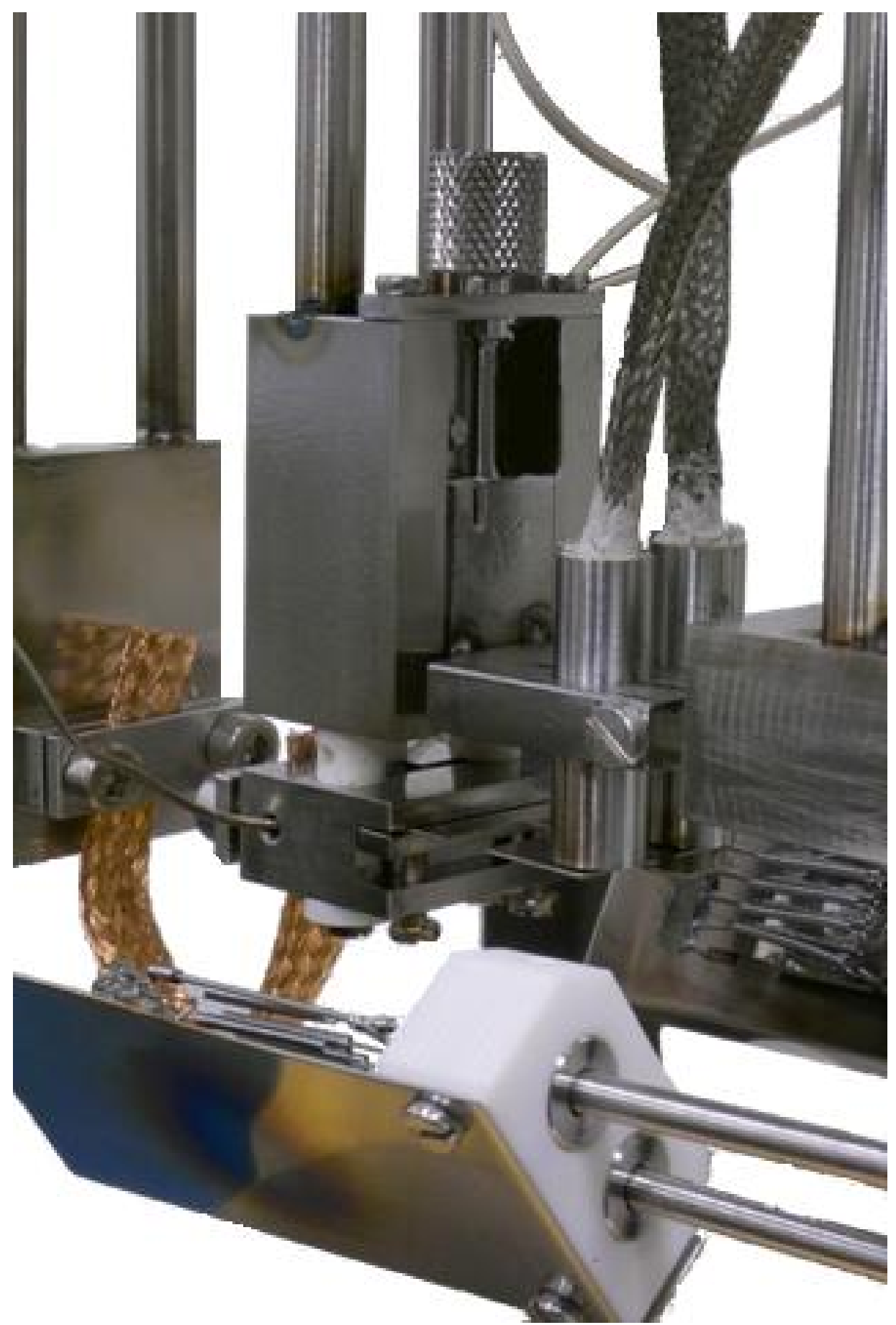

Abbildung 3.2: Detailansicht des Messeinsatzes mit eingebautem Paddel. 
korrekten Paddelkopftemperatur zugeordnet werden. Sofern nicht ausdrücklich anders ausgewiesen, ist diese Temperaturkalibrierung bei allen in Kapitel 4.2 und Kapitel 5 gezeigten Messungen bereits angewendet.

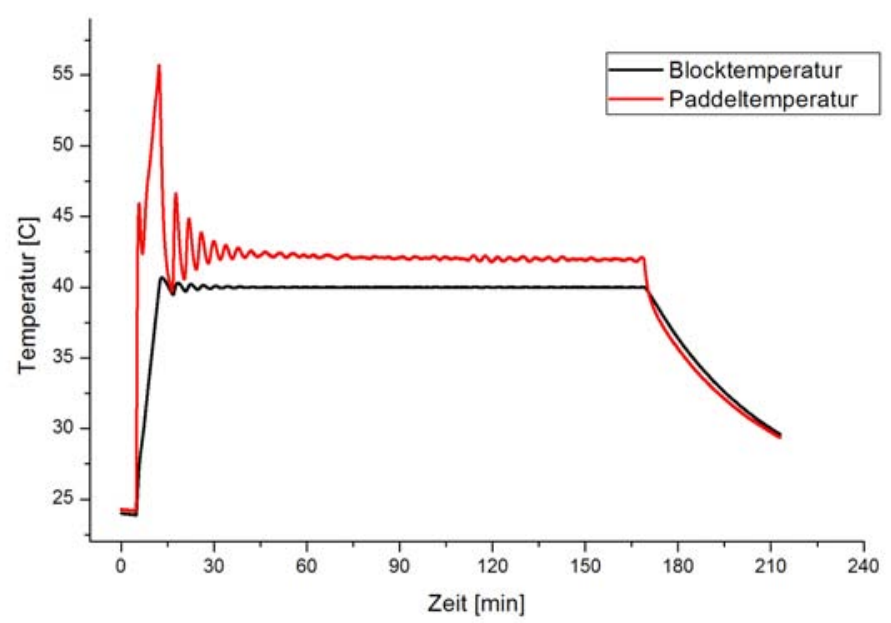

Abbildung 3.3: Zeitlicher Verlauf von Klemmblocktemperatur und Paddelkopftemperatur für die Methode „Anfahren und Warten“.

Während den mechanischen Messungen können Temperaturänderungen des Paddelkopfes indirekt verfolgt werden: Die Resonanzfrequenz der gemessenen AS2-Mode hängt empfindlich von der Paddelkopftemperatur ab. Dies begründet sich aus dem thermischen Erweichen des Siliziums mit steigender Temperatur. Damit fällt die Resonanzfrequenz in erster Näherung linear mit typischerweise $1 \mathrm{~Hz}$ pro $7 \mathrm{~K}$ Temperaturerhöhung.

Für das unbedampfte Paddel ist die Resonanzfrequenz ein derart guter Indikator für die tatsächliche Paddelkopftemperatur, dass mit dieser Frequenz alle gemessen Dämpfungskurven miteinander verglichen werden können. Umgekehrt bedeutet dies jedoch auch, dass die Reproduzierbarkeit der Paddelkopftemperatur von essentieller Bedeutung ist, wenn aus der Änderungen der Resonanzfrequenz von leerem und bedampftem Paddel Rückschlüsse auf das Schermodul der dünnen Schichten gemacht werden sollen. Zu diesem Punkt gibt es weitere Erörterungen in Kapitel 2.4.4.

Das Anfahren der Messtemperaturen erfolgt auf drei Arten:

1) Anfahren und Warten: Da das Paddel eine geringere thermische Trägheit als der Klemmblock besitzt, überschießst die Paddelkopftemperatur den Sollwert deutlich, zumal der Regelkreis auf den Klemm- 
block optimiert ist. Die Solltemperatur muss mit einer geringen Rate von 1-2 $\mathrm{K} / \mathrm{min}$ angefahren werden, um eine Überhitzung der amorphen Schicht zu vermeiden. In dieser Arbeit wird diese Methode nur für eine ausgewählte Temperatur von $40,00{ }^{\circ} \mathrm{C}$ Blocktemperatur verwendet. Diese Temperatur wird als niedrigste, noch stabil einzuregelnde, Temperatur verwendet, da die Raumtemperatur (RT) auch im klimatisierten UHV-Labor noch zu undefiniert ist. Der zeitliche Verlauf von Paddelkopftemperatur und Blocktemperatur ist in Abbildung 3.3 dargestellt.

Die Paddeltemperatur erreicht kurzfristig deutlich höhere Werte und nähert sich langsam einem Gleichgewichtswert von $42{ }^{\circ} \mathrm{C}$ an. Die Regelabweichungen fallen für das Paddel verglichen mit dem Block deutlich größer aus. Der Block schwankt um maximal 0,01 K, während die Paddeltemperatur um bis zu 0,2 K schwankt. Dadurch, dass über einen längeren Zeitraum gemessen werden kann, ist der Mittelwert der Paddeltemperatur und auch die mittlere Frequenzlage sehr viel genauer bestimmbar.

Die Messungen sowohl der Frequenzlage, als auch der Dämpfung, werden nach einer Einschwingdauer von 45-60 Minuten begonnen und erstrecken sich typischerweise über weitere 60-100 Minuten. Die Auftragung Frequenz gegen Messzeit zeigt dabei das Erreichen des thermischen Gleichgewichtes zuverlässig an (Abb. 3.4).

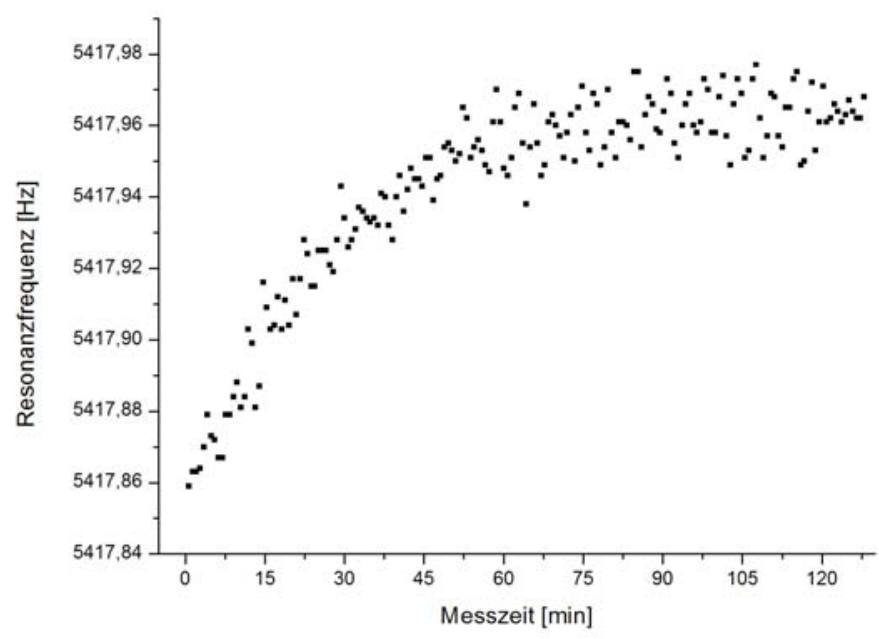

Abbildung 3.4: Messung der Resonanzfrequenz bei $40{ }^{\circ} \mathrm{C}$ Blocktemperatur des leeren Paddels DPO-DB-08. Der Verlauf kann durch thermisches Einschwingen erklärt werden. 
2) Temperaturrampen: In dieser Arbeit sind die meisten Messungen mit ununterbrochenen Temperaturrampen aufgenommen worden. Dabei wird die jeweils erwünschte Maximaltemperatur, beginnend bei Raumtemperatur oder kälter, mit fester Rate mittels PID-Regler (Parameter in Tabelle 3.1) angefahren.

\begin{tabular}{|r|c|c|c|}
\hline & P-Anteil & Integralzeit [s] & Differenzialzeit [s] \\
\hline Silber & 9,75 & 26,77 & 4,46 \\
\hline Edelstahl & 7,13 & 25,85 & 4,31 \\
\hline
\end{tabular}

Tabelle 3.1: Regelparameter der verschiedenen Klemmblöcke.

Während des Heizens ist dabei die Paddelkopftemperatur immer höher als die Blocktemperatur und bei Kühlrampen, zumindest im oberen Temperaturbereich, ebenfalls. Während des Heizens mit konstanter Rate stellt sich nach kurzer Zeit ein ,dynamisches Gleichgewicht" ein, bei dem die Paddeltemperatur fest mit der Blocktemperatur verknüpft ist. Der zeitliche Verlauf von Kopftemperatur und Blocktemperatur für die Rate $1 \mathrm{~K} / \mathrm{min}$ ist in Abbildung 3.5 gezeigt. Man erkennt, dass der Zusammenhang von Block- und Paddeltemperatur im Wesentlichen linear beschreibbar ist. Die tatsächliche Anpassung erfolgte jedoch mit einem Polynom dritter Ordnung, dessen Standardabweichung von den Messdaten nur 0,4 K für den gesamten Bereich beträgt.

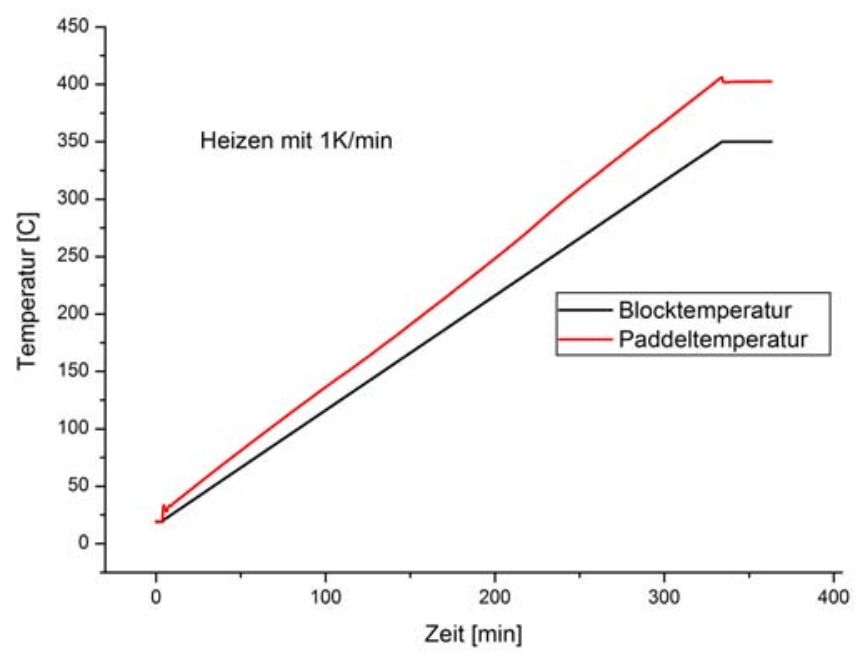

Abbildung 3.5: Temperaturverlauf beim konstanten Heizen auf $350{ }^{\circ} \mathrm{C}$ Blocktemperatur. 
Die Zuverlässigkeit dieser Methode wurde zuvor schon in der Diplomarbeit von M. Tschuschke [62] gezeigt und auch angewendet. In der hier vorliegenden Arbeit musste aber aus praktischen Gründen ${ }^{2}$ mit deutlich höheren Raten von bis zu $3 \mathrm{~K} / \mathrm{min}$ gearbeitet werden, während in der zitierten Arbeit mit weit weniger als $1 \mathrm{~K} / \mathrm{min}$ gefahren wurde.

Die Rampen haben den Vorteil, dass ohne Unterbrechung und Wartezeiten kontinuierlich gemessen werden kann. Während des ersten Aufheizens einer frisch hergestellten amorphen Probe tritt eine Relaxation auf, die zumeist als Alterung bezeichnet wird (siehe Abschnitt 1.3.3, bzw. [31]). In dieser Arbeit wird dieser Effekt für dünne Schichten gezielt untersucht. Um die Relaxation direkt beobachten zu können, ist eine hohe Anzahl von Messpunkten ohne großen Zeit- und Temperaturabstand notwendig.

Für die Dämpfungsmessungen ist der Zeit zwischen zwei Messungen durch die ring-downs des Paddels eine untere Grenze gesetzt. Um das Paddel auf 1/4 der getriebenen Amplitude ausschwingen zu lassen, und anschließend wieder auf eine Amplitude einschwingen, die der Anregung entspricht, benötigt es (stark Güte-abhängig) typischerweise eine Minute. Zusammen mit einer Heizrate von $1 \mathrm{~K} / \mathrm{min}$ während der Messung, ist damit eine hohe Rasterdichte an Messpunkten im Temperaturverlauf gewährleistet.

3) Manuelles Kühlen: Zum Erreichen von Temperaturen unterhalb von RT ist der neue Messeinsatz mit einer Durchführung für flüssigen Stickstoff und einem an den Klemmblock thermisch gekoppelten Kühlblock ausgestattet. Durch diesen Kühlblock kann permanent flüssiger Stickstoff gepumpt werden. Zur Vermeidung von akustischen Emissionen, die die Messung stören können, hat sich aber eine andere Technik bewährt: Die Zu- und Ableitung des flüssigen Stickstoffs bilden ein langes U-Rohr. Dieses U-Rohr wird mit flüssigen Stickstoff gefüllt und immer wieder nachgefüllt. Sobald der Kühlblock Stickstoffsiedetemperatur erreicht hat, muss nur noch selten nachgefüllt werden.

Die Kopplung von Paddelkopftemperatur und gemessener Klemmblocktemperatur kann auch hierbei an der Paddelresonanzfrequenz verfolgt werden. Sobald keine signifikante Temperaturerniedrigung am Klemmblock mehr erreicht werden kann, lässt man den Stickstoff vollständig verdampfen, worauf die Temperatur langsam wieder ansteigt. An dieser Stelle setzt eine Temperaturrampe wie zuvor beschrieben an. Dieses

\footnotetext{
${ }^{2}$ Hohe Heizraten begünstigen die Beobachtbarkeit von Alterungseffekten, zudem sollten vergleichbare Heizraten wie in [7] verwendet werden.
} 
Verfahren hat sich ebenfalls als reproduzierbar erwiesen, jedoch ist eine andere Temperatureichung vorzunehmen als beim reinen Heizzyklus mit gleicher Rate, beginnend oberhalb von Raumtemperatur.

\subsubsection{Datenaufnahme}

Ergänzend zu Kapitel 2.4 wird hier die technische Realisierung einer mechanischen Messung mittels Doppelpaddel, die elektrische Seite des Messaufbaus und die genaue Vorgehensweise kurz beschrieben.

\section{Zur Vermessung einer dünnen Schicht erfolgen folgende Schritte:}

- Ein neues Doppelpaddel wird, wie in Kapitel 3.2.2 dargestellt, vorbereitet und in den Messaufbau eingebaut. Die Justage des Elektrodenabstandes erfolgt nach Augenmaß und muss ggf. korrigiert werden. Der Messaufbau wird zunächst mit Viton ${ }^{\circledR}$ gedichtet in die UHV - Anlage eingebaut und evakuiert.

- Über einen Frequenzscan wird das Spektrum des verwendeten Doppelpaddels aufgenommen, insbesondere wird die AS2-Mode identifiziert. Die AS2-Mode wird manuell angeregt und der PLL (beschrieben auf Seite 45) eingestellt und getestet. Aus dem Verhältnis von Anregungsstärke/ Resonanzamplitude kann der zuvor eingestellte Abstand überprüft werden.

- Das Paddel wird zur Beseitigung von Oberflächenkontaminationen auf $250{ }^{\circ} \mathrm{C}$ geheizt, anschließend erfolgen Testmessungen zur Überprüfung der Klemmung, der Messeinstellung und Paddelbesonderheiten. Tritt ein Problem auf (zumeist klemmungsbedingt) erfolgt ein Neueinbau oder die Verwendung eines anderen vorbereiteten Paddels. Andernfalls wird der Einsatz mit einer Kupferdichtung fest eingebaut.

- Ausheizen der UHV-Kammer: Das Paddel wird auf eine Temperatur von $250{ }^{\circ} \mathrm{C}$ geheizt, anschließend beginnt das Ausheizen der UHVKammer. Endtemperatur beim Ausheizen: $250{ }^{\circ} \mathrm{C}$, Dauer: 3-5 Tage, Enddruck bei beheizter Anlage $\approx 3 * 10^{-7}$ mbar. Basisdruck nach dem Ausheizen: $1,5-2,0 * 10^{-9}$ mbar.

- Das Paddel wird abschließend auf $350{ }^{\circ} \mathrm{C}$ Blocktemperatur geheizt um letzte Gaskontaminationen zu entfernen. 
- Es erfolgen die Leermessungen. Es werden sowohl die Messungen während Temperaturrampen bis $350{ }^{\circ} \mathrm{C}$ Blocktemperatur als auch die Messungen bei konstanter Temperatur von $40{ }^{\circ} \mathrm{C}$ mehrfach wiederholt. $\mathrm{Zu}$ Beginn zeigen sich dabei stets Änderungen zwischen den Messungen, diese können aber durch wiederholte thermische Zyklen beseitigt werden. Sobald aufeinander folgende Messungen keine Unterschiede mehr aufweisen ist das Paddel bereit zum Beschichten.

- Die gewünschte Schicht wird wie in Kapitel 3.3.2 beschrieben auf das DPO und das Referenzsubstrat gedampft.

- Das Referenzsubstrat wird ausgeschleust und die Dicke, Amorphizität, Zusammensetzung und in einigen Fällen auch die Oberflächenmorphologie der aufgedampften Schicht untersucht (Kap.3.4).

- Das Paddel mit der Schicht wird zuerst bei $40{ }^{\circ} \mathrm{C}$ gemessen, um Schichtdämpfung und das Schermodul zu bestimmen. Bei guter Kenntnis der Schichtdicke kann daraus bereits auf die Mikrostruktur der Probe geschlossen werden.

- Es erfolgt die eigentliche Heizmessung der amorphen Probe mit konstanter Rate, die im Folgenden auch als Kristallisationsmessung bezeichnet wird, da während dieser stets eine Kristallisationssignatur zu beobachten ist.

- Anschließend wird erneut bei $40{ }^{\circ} \mathrm{C}$ gemessen, um Schichtdämpfung und Schermodul der kristallinen Schicht zu bestimmen.

- Um auch das Verlustspektrum der kristallinen Schicht mit der amorphen vergleichen zu können, wird eine letzte Heizmessung mit dem gleichen Temperaturintervall und Rate wie bei der Kristallisationsmessung vorgenommen.

- In einzelnen Fällen wurde die kristalline Schicht einige Zeit bei $400{ }^{\circ} \mathrm{C}$ gehalten und damit Kornwachstum und andere Rekristallisationsprozesse weitestgehend abzuschließen, um anschließend das Paddel erneut als Basis für eine amorphe Schicht zu verwenden. Dieses Verfahren hatte sich in der Doktorarbeit von P. Rösner [6] bewährt, brachte aber für die hier verwendeten sehr dünnen Schichten Probleme mit sich (diskutiert in Kapitel 5.1.2). Um die Interpretation der Messdaten zu vereinfachen, wurde für jede weitere Schicht wieder mit einem neuen Doppelpaddel begonnen. 


\section{Die Messelektronik:}

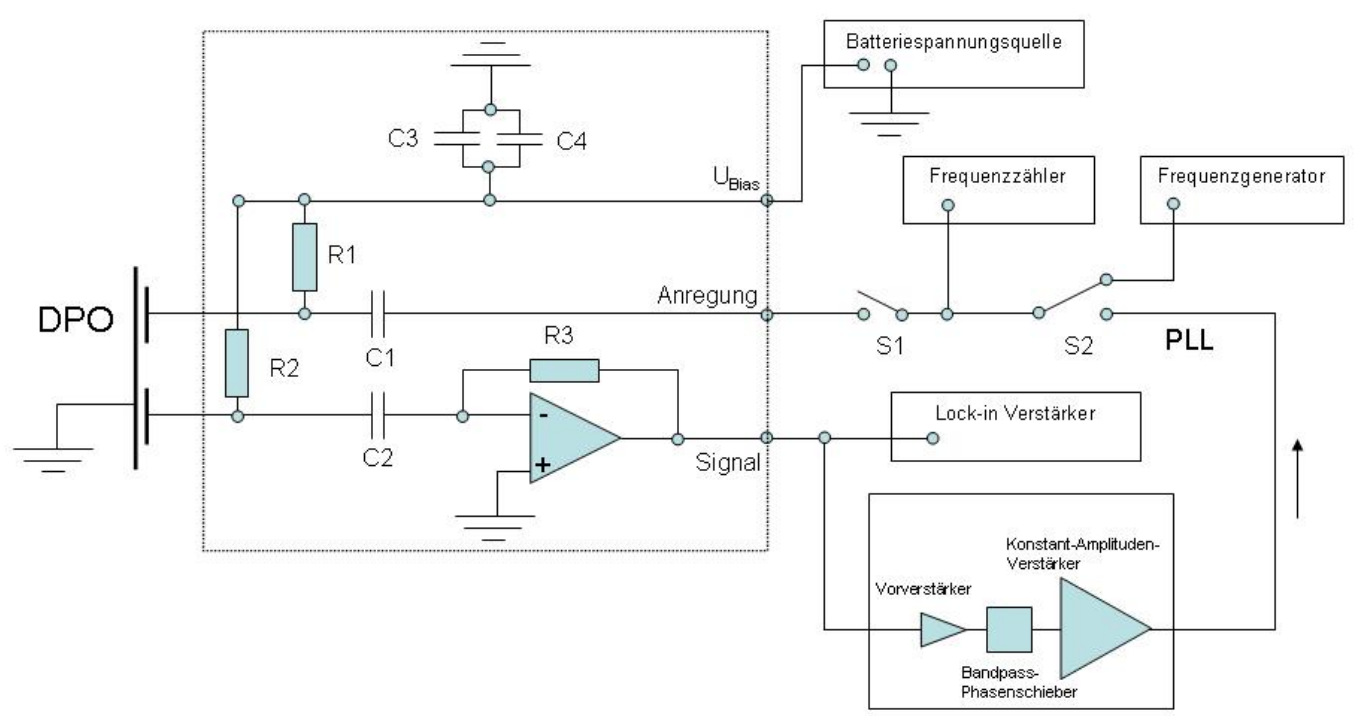

Abbildung 3.6: Vereinfachte Darstellung der verwendeten Schaltung.

Die verwendete Messelektronik wurde in wesentlichen Teilen von P. Rösner bzw. M. Tschuschke übernommen. Es wurden zu Beginn dieser Arbeit zusammen mit Prof. R. Richert ${ }^{3}$ und A. Meschede ${ }^{4}$ einige Verbesserungen vorgenommen. Zur Eliminierung von Masseschleifen und anderen elektrischen Störungen wurde ein neuer Temperaturcontroller mit TCP/IP - Schnittstelle in Betrieb genommen, die erste Signalverstärkungsstufe (in Abb. 3.6 gestrichelt eingerahmt) komplett neu aufgebaut und die Massepunkte an der UHV-Anlage zusammengelegt. Damit ergibt sich eine sehr störungsarme Signalaufnahme, verbleibende Störungen sind überwiegend akustischer Art.

Obwohl die verwendete Schaltung bereits in [58] und [6] ausführlich beschrieben ist, soll der hier verwendete Aufbau noch einmal anhand der Abbildung 3.6 kurz erläutert werden:

Die Elektroden bilden mit dem Doppelpaddel jeweils einen Kondensator. Diese werden über die Spannung $U_{\text {Bias }}$ auf ca. 140 Volt geladen. Die Widerstände R1 und R2 begrenzen mit jeweils $10 \mathrm{M} \Omega$ den Stromfluss der Batterie und verhindern das Abfließen des Messignales auf der Signalseite. Die Kondensa-

\footnotetext{
${ }^{3}$ Department of Chemistry \& Biochemistry, Arizona State University, U.S.A.

${ }^{4}$ Institut für Materialphysik IMP, Universität Göttingen
} 
toren $\mathrm{C} 3$ und $\mathrm{C} 4(0,68 \mu \mathrm{F}$ und $100 \mathrm{pF})$ halten einerseits die Ladung konstant und lassen hochfrequente Störungen abfließen. Die Kondensatore C1 und C2 (10 nF, keramisch) trennen die Bias-Spannung nach außen ab. Über den Anschluss „Anregung“" wird mit dem Frequenzgenerator eine Wechselspannung mit $\approx 0,3 \mathrm{~V}$ der Gleichspannung auf der Anregungselektrode überlagert. Durch die resultierende Abstoßungskraft gleicher Frequenz wird das DPO in Schwingung versetzt.

Diese Schwingung ändert periodisch den Abstand und damit die Kapazität bei fester Potentialdifferenz $U_{\text {Bias }}$. Daraus ergibt sich ein Verschiebungsstrom, der die Paddelschwingungsamplitude (phasenverschoben) anzeigt. Der direkt nachgeschaltete sehr rauscharme und hochohmige Operationsverstärker vom Typ AD-549L ist als Strom-Spannungswandler mit R3 = $10 \mathrm{M} \Omega$ beschaltet und liefert damit $10^{7} \mathrm{~V} / \mathrm{A}$. Das Spannungssignal beträgt anschließend typischerweise $30 \mathrm{mV}$ und wird mit einem Lock-in-Verstärker gemessen. Um das Paddel dauerhaft in seiner Eigenfrequenz zu betreiben wird ein „pseudo-PLL“ (phase-locked loop) verwendet. Dazu wird das Antwortsignal des angeregten DPOs phasengeschoben und geeignet verstärkt als Anregung zurückgeführt. Hierfür wird der Schalter S2 umgelegt. Dieser Rückkopplungskreis funktioniert ähnlich wie eine Rückkopplung von Mikrophon und Lautsprecher. Die Verstärkung des Kreises ist maximal bei der Resonanzfrequenz des Doppelpaddels, wodurch sich diese selbst stabilisiert. Die Resonanzfrequenz ist wegen der geringen Paddeldämpfung quasi identisch mit der Eigenfrequenz. Die Paddelamplitude, die sich im eingeschwungenem Fall einstellt, ist direkt abhängig von der Verstärkung des Konstant-Amplituden-Verstärkers und der Paddeldämpfung. Ändert sich die Paddeldämpfung deutlich, so muss die Anregungsamplitude des Rückkopplungskreises manuell angepasst werden, um die Paddelschwingungseigenschaften bei etwa konstanter Amplitude zu messen.

Die temperaturabhängige Resonanzfrequenz des Paddels wird stets nur im eingeschwungenem PLL mit dem Frequenzzähler der Anregungsseite gemessen. Da der quasi-PLL der Resonanzfrequenzänderung beim Heizen des Paddels folgt, kann diese ständig gemessen und überwacht werden. Um nun die Dämpfung zu messen wird ein , Ring-down“ aufgenommen, indem der Schalter S1 geöffnet wird. Ohne Anregung schwingt das DPO frei aus, die Amplitude als Funktion der Zeit wird mit dem Lock-in Verstärker gemessen. Beendet man den „Ring-down" bei 1/4 der eingeschwungenen Amplitude durch Schließen von S1, so kann der PLL meistens direkt wieder aufgenommen werden. Ist dieses nicht erfolgreich so wird das Paddel mit dem Frequenzgenerator angeregt (S2 auf FG, korrekte Frequenz wird durch Extrapolation berechnet) und anschließend der PLL wieder hergestellt (S2 auf PLL). Während des Ring-downs und dem Wiederanlaufen wird keine Frequenz gemessen. 


\subsection{Herstellung, Vorbereitung der DPO}

\subsubsection{Herstellung}

Doppelpaddel-Oszillatoren sind bisher nicht standardisiert und kommerziell erhältlich. Daher ist ihre Fertigung und Bearbeitung hier kurz beschrieben: Die in dieser Arbeit verwendeten Doppelpaddel wurden sämtlich mittels Laserschneidetechnik aus Silizium-Wafern herausgeschnitten. Dieser Arbeitsschritt erfolgt im Rahmen einer Kooperation im Fraunhofer Institut für Lasertechnik in Aachen. Die Abmaße sind aus der Abbildung 3.7 ersichtlich. Die genauen Maße sind in [6] aufgelistet.

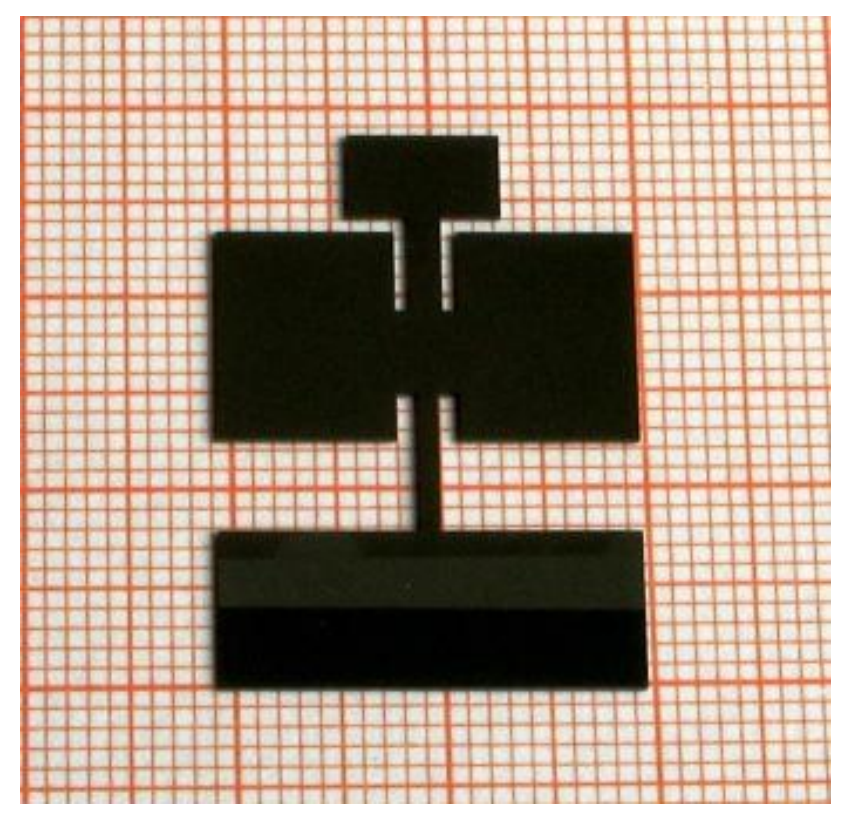

Abbildung 3.7: Aufsicht auf einen bedampften Doppelpaddel-Oszillator auf Millimeterpapier.

Zum Einsatz kamen verschiedene Chargen von Si- Wafern, die alle die hier aufgelisteten Spezifikationen erfüllen:

- Siliziumwafer, hergestellt im FZ (floating zone)- Verfahren, undotiert, spezifischer Widerstand $\geq 3 \mathrm{~K} \Omega$.

- Orientierung: (100), doppelseitig poliert.

- Durchmesser: 3 Zoll, Dicke: 250 - $300 \mu \mathrm{m} .{ }^{5}$

\footnotetext{
${ }^{5}$ Die tatsächliche Dicke eines Wafers schwankt auch innerhalb einer Charge. Dies ändert aber im Wesentlichen nur die genaue Lage der Eigenmoden.
} 
Aus einem solchen Wafer können vier Oszillatoren geschnitten werden. Bedingt durch den Laserschneideprozess sind die Kanten der Oszillatoren sehr rau, wie in Abbildung 3.8 und 3.9 deutlich gezeigt wird. In der Arbeit von P. Rösner [6] zeigten nass-chemisch heraus geätzte Paddel deutlich geringere Grunddämpfung als lasergeschnittene. Daher wurden der Versuch unternommen, mit einer Ätz-Nachbehandlung mittels heißer KOH-Lösung, die Kanten zu glätten. Dies zeigte jedoch bereits qualitativ keine Verbesserung und wurde nicht weiterverfolgt.

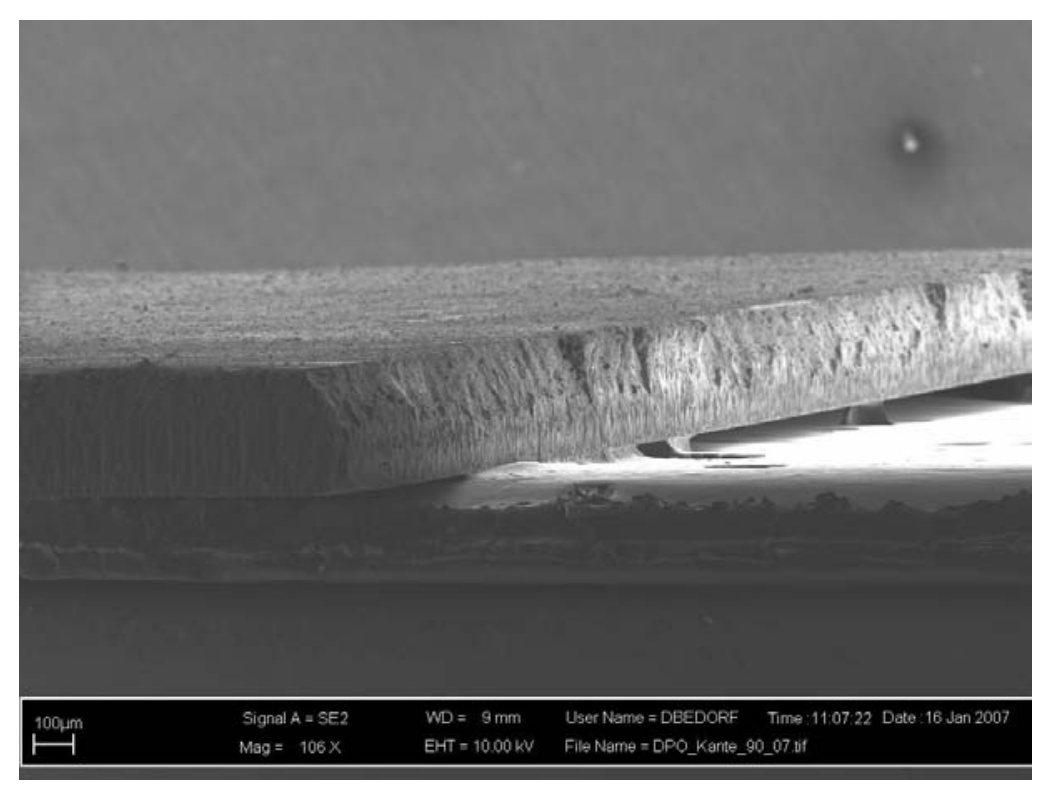

Abbildung 3.8: REM-Aufnahme eines lasergeschnittenen Paddels vor der Reinigung.

\subsubsection{Vorbereitung}

Der Schneideprozess führt zu Materialauftrag auf die Oberflächen der Paddel. Dieser kann nur mechanisch wieder entfernt werden. Dazu wird eine Vorreinigung mit einem Glasfaserpinsel durchgeführt. Anschließend erfolgt eine Reinigung mit Aceton im Ultraschallbad. Abschließend wird jedes Paddel mit reinstem Methanol gereinigt und getrocknet. Teilweise erweisen sich die Verschmutzungen als so hartnäckig, dass weitere Reinigungsschritte dazwischen nötig sind: Wattestäbchen mit 2-Propanol, Tauchbad in Dichlormethan, und in extremen Fällen: Kochende Spülmaschinenlauge oder $\mathrm{H}_{2} \mathrm{SO}_{4}$ mit $\mathrm{H}_{2} \mathrm{O}_{2}$ (Piranha-Lösung) und anschließendes Spülen mit destilliertem Wasser. 


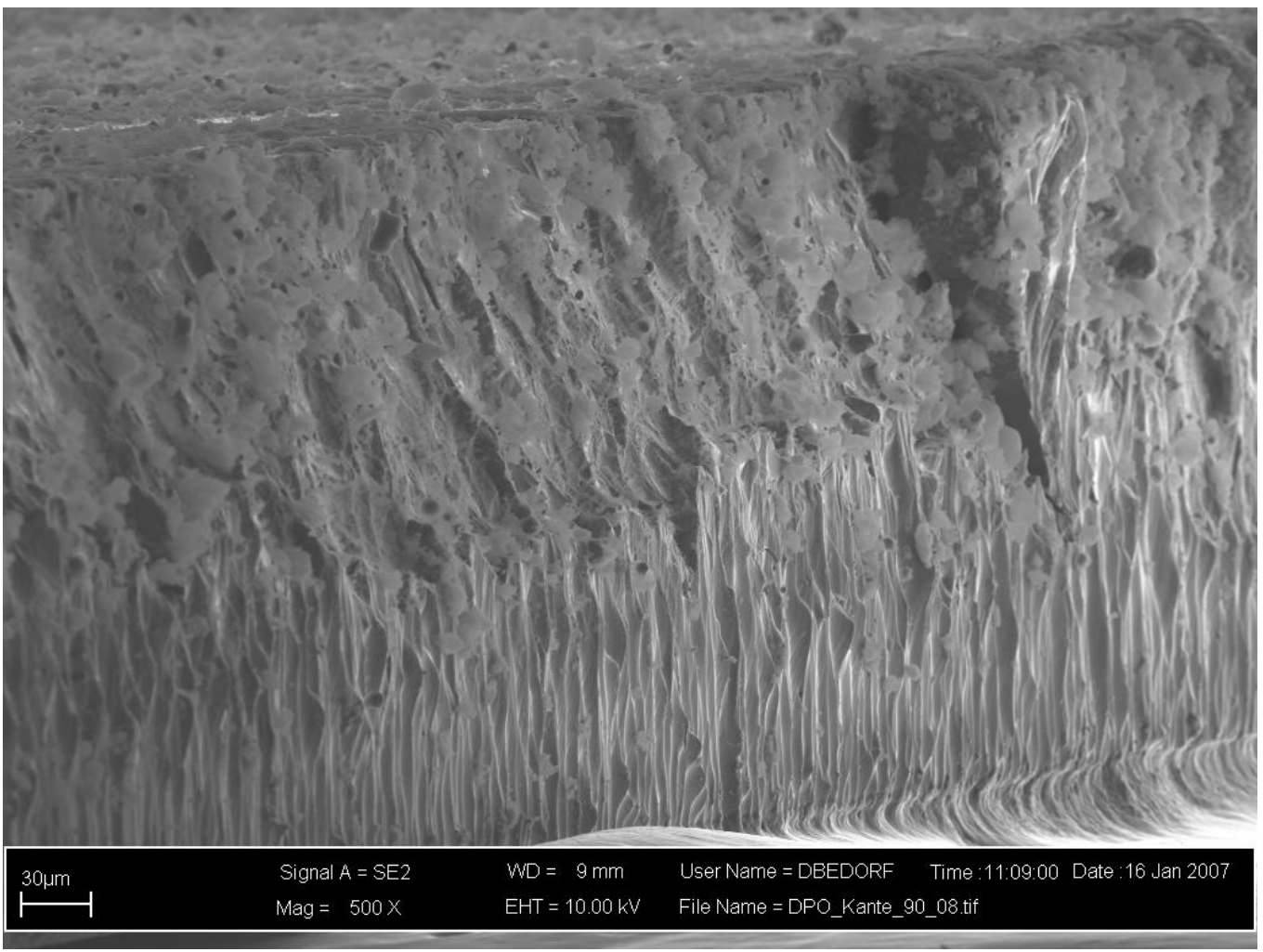

Abbildung 3.9: REM-Aufnahme der Kantendetails nach dem Laserschneiden. Man erkennt die Bahnen, die der Laserstrahl aufgeschmolzen hat.

Die so gereinigten Paddel werden vor dem Einbau in die Messanlage noch beidseitig mit $50 \mathrm{~nm}$ Tantal beschichtet.

Die Tantalschicht wird durch einen Sputterprozess in einer Beschichtungsanlage ${ }^{6}$ hergestellt. Die Sputterparameter sind: Basisdruck: $1^{*} 10^{-7}$ mbar, Sputterdruck: $1^{*} 10^{-3}$ mbar Argon, Leistung 750 W. Diese Parameter ermöglichen sehr glatte Schichten, wie sie als Unterlage für die dünnen Messschichten benötigt werden.

Die Tantalschicht übernimmt später mehrere Funktionen gleichzeitig:

- Die Tantalschicht auf der oberen, den Mess-Elektroden zugewandten Seite, bildet einen elektrisch fest definierten Gegenkontakt. Die Paddeloberseite ist über den Klemmfuß mit der Masse verbunden. Ohne diese Gegenelektrode entsteht, durch das nur sehr gering leitfähige Silizium,

${ }^{6}$ ET PLASMAFAB MS5200 
ein Übersprechen zwischen den beiden Elektroden. Zuvor wurde dieser Kontakt stets mit einer Goldschicht auf Chrom hergestellt, jedoch tendieren diese Schichten bei sehr hohen Temperaturen zur Entnetzung. Die Tantalschicht dagegen ist thermisch sehr stabil.

- Die Tantalschicht auf der Unterseite dient primär als Diffusionsbarriere. In der Vorgängerarbeit [6] konnte gezeigt werden, dass durch eine Tantalbarriere die Eindiffusion von Kupfer aus den $\mathrm{ZrAlCu}-\mathrm{Schichten}$ in das Silizium verhindert ist. Auch in dieser Arbeit ist Kupfer Bestandteil der Schichtlegierung und es zeigten sich ebenfalls Änderungen der Paddeldämpfung nach längerem thermischen Auslagern der Schicht. Diese können mit der Tantal-Barriere verhindert werden.

- Des Weiteren erweist sich die Tantalschicht als nützlich um die Temperatur-Reproduzierbarkeit nach dem Aufdampfen der eigentlich zu messenden Schicht zu verbessern. Im Strahlungsfeld der Lampenheizung ist die Paddeltemperatur auch vom Albedo der Paddelunterseite abhängig. Der Unterschied einer (infrarot-transparenten) Silizium-Oberfläche zur amorphen metallischen Schicht dürfte groß ausfallen. In einem Versuch mit einem völlig unbedampften Siliziumpaddels als Leerpaddel war die Temperaturreproduzierbarkeit nach dem Aufdampfen der metallischen Schicht nicht mehr gegeben. Die Tantalschicht jedoch erscheint optisch fast ununterscheidbar von der zu untersuchenden PdCuSi-Schicht.

\subsection{Herstellung dünner PdCuSi-Schichten}

Um die zu untersuchenden sehr dünnen PdCuSi-Schichten herzustellen und anschließend $\mathrm{zu}$ vermessen wird eine UHV-Aufdampfkammer mit dem im Kapitel 3.1 beschriebenen Mess- und Beschichtungseinsatz verwendet.

\subsubsection{Die Balzers UHV-Anlage}

Die hier verwendete Balzers UHV-Anlage wurde zu Beginn dieser Arbeit in Teilen umgebaut und soll nur sehr kurz beschrieben werden:

Die Kammer enthält drei Elektronenstrahlverdampfer mit denen die drei Komponenten Kupfer, Silizium und Palladium einzeln verdampft werden können. Über jedem Elektronenstrahlverdampfer ist eine Schwingquarzwaage eingebaut, um die einzelnen Aufdampfraten messen und regeln zu können. Um nur den jeweiligen Verdampfer zu messen, sind an den Schwingquarzen 
Blenden angebracht, die eine Fremdbedampfung verhindern. Das Doppelpaddel befindet sich oberhalb der Verdampfer geometrisch zentriert. Direkt daneben ist das Referenzsubstrat aufgehängt. Der Referenzsubstrathalter kann über eine seitlich angebrachte Schleusenkammer in die Schleuse, bzw. in die Kammer geschleust werden, ohne das Vakuum zu brechen.

Das Pumpsystem der Hauptkammer besteht aus einer Turbopumpe, einer Ionen-Getter-Pumpe, einer Titan-Sublimationspumpe (TSP) und einer Stickstoff-Kühlfalle. Damit ergibt sich ein Basisdruck von 1,5-2,0*10-9 mbar. Kurzfristig kann mit der Kühlfalle und TSP-Einsatz etwa $2-5^{*} 10^{-10}$ mbar ermöglicht werden.

\subsubsection{Der Aufdampfprozess}

Für das Bedampfen werden Reinstmaterialien, jeweils geeignet vorgereinigt, in die Elektonenstrahlverdampfer vorgelegt. Die verwendeten Materialien sind in Tabelle 3.2 aufgelistet.

\begin{tabular}{|l|c|c|c|}
\hline Element: & Reinheit: [\%] & Hersteller: & Form: \\
\hline Palladium & 99,95 & $\begin{array}{l}\text { Alfa Aesar } \\
\text { Goodfellow }\end{array}$ & $\begin{array}{c}\text { Stab 6,35 } \mathrm{mm} \oslash \\
\text { Stab 6,00 mm } \oslash\end{array}$ \\
\hline Silizium & 99,999 & E. Wagener & Stücke \\
\hline Kupfer & 99,999 & Goodfellow & Granulat \\
\hline
\end{tabular}

Tabelle 3.2: Verwendete Materialien zum Aufdampfen.

Die Materialien werden zunächst einzeln unter UHV-Bedingungen aufgeschmolzen um sie zu entgasen und um eine gleichförmige Pillenform zu erhalten. Um den geometrischen Faktor zu bestimmen, der das Verhältnis von Schichtdicke auf dem Schwingquarz zu Schichtdicke auf dem Paddel angibt, werden Tooling-Schichten der jeweiligen Materialien hergestellt und mit Kleinwinkel-Röntgen die Dicke vermessen. Nach Änderungen des Füllstandes oder der Pillenform der Materialien im Tiegel werden solche Messungen wiederholt. Die Legierungsschicht entsteht durch gleichzeitiges Verdampfen und Kondensieren der drei Elemente mit dem korrekten Ratenverhältnis. Jede Einzelrate wird durch einen Regelkreis auf dem gewünschten Wert stabil gehalten.

Die Zusammensetzung der Schicht berechnet sich wie folgt:

Die in der Zeit $t$ aufgedampfte Masse $M_{A}$ einer Komponente A auf eine Fläche $F$ mit Rate $r_{A}$ berechnet sich gemäß:

$$
M_{A}=r_{A} t F \rho_{A}
$$


Damit ergibt sich für den Massenanteil $w_{A}$ der Komponente A an einer mehrkomponentigen Schicht:

$$
w_{A}=\frac{r_{A} t F \rho_{A}}{\sum r_{i} t F \rho_{i}}
$$

Nun können zunächst $t$ und $F$ gekürzt werden, zudem stellt die Summe $\sum r_{i} \rho_{i}$ bei vorgegebener Gesamtaufdampfrate eine Konstante dar, die mit $R_{G}$ ersetzt werden kann. Die jeweiligen Einzelraten berechen sich damit gemäß:

$$
r_{i}=\frac{w_{i}}{\rho_{i}} R_{G}
$$

Der Parameter $R_{G}$ wird so gewählt, dass die kleinste Rate, die des Kupfers, noch stabil eingeregelt werden kann.

Sobald alle Materialien die vorgegebene Aufdampfrate erreicht haben, beginnt der Aufdampfprozess mit dem Öffnen des Shutters unter dem Paddel und dem Referenzsubstrat. Das Erreichen der vorgegeben Dicke für Palladium, der Hauptkomponente, gibt das Signal zum Schließen des Shutters. Alle weiteren Schichtdicken auf den Schwingquarzwaagen und die Aufdampfzeit werden ebenfalls protokolliert, um den Aufdampfprozess zu dokumentieren bzw. auf mögliche Abweichungen zu überprüfen.

\subsection{Weitere Analysen dünner Schichten}

Die Analysen, die nicht-mechanischer Natur sind, werden überwiegend an den Referenzsubstraten durchgeführt, die gleichzeitig mit dem Doppelpaddel identisch beschichtet werden. Um diese Analysen zu vereinfachen, sind die Referenzsubstrate jedoch nicht mit Tantal belegt, zudem kommen teilweise andere Substrate zum Einsatz ${ }^{7}$.

\subsubsection{Kleinwinkel-Röntgenstreuung}

Die Verwendung der Kleinwinkel-Röntgenstreuung dient zu allererst der Bestimmung der Schichtdicke. In üblicher Bregg-Bretano $(\Theta-2 \Theta)$-Geometrie wird die Röntgen-Reflektivität bei kleinen Winkeln $\left(0,8^{\circ} \leq 2 \Theta \leq 4,5^{\circ}\right)$ bestimmt. Dazu wird ein D8-Advance Röntgendiffraktometer der Firma Bruker aXS verwendet. Die Röntgenstrahlung wird durch einen Göbelspiegel monochromatisiert, so dass nur die Kupfer $\mathrm{K}_{\alpha}$ mit $\lambda=1,54148 \AA$ auf die

\footnotetext{
${ }^{7}$ Ein einfaches Si-Substrat verhindert beispielsweise die Bestimmung der Zusammensetzung, da Silizium auch Schichtbestandteil ist.
} 
Probe trifft. Für sehr kleine Winkel tritt Totalreflexion auf, ab einem kritischen Winkel nimmt die Reflektivität schnell ab. Dieser Abfall ist durch Oberflächen- und Genzflächenrauigkeiten bestimmt. Bedingt durch konstruktive Interferenz von reflektierten Strahlen an Oberfläche und Substratgrenzfläche treten periodisch Maxima in der Reflektivität auf. Diese Abstände $\Delta \Theta$ sind mit der Schichtdicke D näherungsweise verknüpft über:

$$
D=\frac{\lambda}{2 \Delta \Theta}
$$

Die gemessenen Reflektivitätskurven können mit der auf dem Parrat-Algorithmus [63] basierenden Software Parrat [64] simuliert werden. Aus der Anpassung der Modellparameter an die Messkurven werden hier nur Schichtdicke und Oberflächenrauigkeit weiter behandelt. Nach veränderten Schichtdicken bei gleicher Stöchiometrie sind dies auch die einzigen Parameter, die für jede Schicht neu angepasst werden.

\subsubsection{Weitwinkel-Röntgenstreuung}

Die Weitwinkel-Röntgenstreuungsexperimente werden ebenfalls an dem Diffraktometer D8-Advance durchgeführt. Dabei wird die Mikrostruktur der Schichten geprüft. Die Messungen erfolgen ebenfalls in $(\Theta-2 \Theta)$-Geometrie. Um keine scharfen und intensiven Beugungsreflexe der einkristallinen Substrate in den weit geöffneten Detektor zu bringen, wird die Probe ein wenig verkippt. Damit kann eine amorphe oder polykristalline Schicht weiterhin gemessen werden.

In der Weitwinkel-Röntgenbeugung beobachtet man für kristalline Materialien mit genau definierten Netzebenen scharfe Reflexe, die der allgemein bekannten Bragg-Bedingung genügen. In amorphen Materialien ohne periodische Struktur führt nur die Nahordnung der dichten Kugelpackung der Atome zu charakteristischen Abständen der Streuzentren. Dieses resultiert in breiten, wenig intensiven Beugungsmaxima [3]. Der Nachweis dieser Maxima ist für sehr dünne amorphe Schichten schwierig, da das Signal der dünnen Schichten klein wird gegenüber der Untergrund-Intensität bzw. den Defekten der Substrate.

\subsubsection{Rasterelektronenmikroskopie mit EDX}

Das Rasterelektronenmikroskop LEO SUPRA 35 ist mit einer EDX (energy dispersive X-ray spectroscopy) - Analyse-Einheit ausgestattet, die es ermöglicht die Zusammensetzung der Schichten zu überprüfen. Dieses Verfahren wurde auf alle hergestellten Legierungsschichten angewendet. 
In einigen Fällen kam auch die mikroskopische Abbildung zum Einsatz: Bei einem Rasterelektronenmikroskop (REM) rastert ein Elektronenstrahl die Probe lateral ab. Dabei werden u. a. Rückstreu- und Sekundärelektronen aus der Probe herausgeschlagen. Die Intensität hängt dabei von dem Material und der lokalen Beschaffenheit der Probe ab. Mit Elektronendetektoren wird diese „Helligkeit" gemessen und räumlich zugeordnet. So entsteht ein überwiegend topographisches Bild ohne quantitative Höheninformation.

Zur Elementaranalyse mittels EDX wird die Probe von einem intensiven Elektronenstrahl mit $20 \mathrm{kV}$ Beschleunigungsspannung in dem zu untersuchendem Areal gerastert. Durch inelastische Stöße der schnellen Elektronen mit gebundenen Elektronen der inneren Schalen entsteht auch charakteristische Röntgenstrahlung. Diese Strahlung wird als Funktion der Energie gemessen, es ergeben sich charakteristische Spektren. Zur quantitativen Auswertung wird eine mitgelieferte kommerzielle Software verwendet, die die elementspezifischen Ionisationswahrscheinlichkeiten berücksichtigt. Zu Beachten ist jedoch, dass der Elektronenstrahl durch eine Eindringtiefe von einigen $\mu \mathrm{m}$ im Falle von dünnen Schichten im Wesentlichen Informationen über das Substrat liefert.

\subsubsection{Rastersondenmikroskopie}

Die Rastersondenmikroskopie ist mittlerweile eine weit verbreitete Methode, die sehr oft beschrieben worden ist (z. B. [65, 66, 67]).

Für Messungen in dieser Arbeit wurde die Rastertunnelmikroskopie (STM, Scanning tunneling microscopy) und Rasterkraftmikroskopie (AFM, Atomic force microscopy) verwendet, um die Rauigkeit der Schichten bzw. der Substrate zu kontrollieren ${ }^{8}$.

STM: Messprinzip: In einem STM rastert eine scharfe leitfähige Spitze sehr nah über eine Probenoberfläche. Bei einer kleinen angelegten Spannung zwischen Spitze und Probe fließst auch berührungslos ein sehr kleiner Tunnelstrom, der exponentiell vom Abstand abhängt. Im Bildmodus wird dieser Strom durch Regelung des Abstandes mittels eines Piezo-Scanners konstant gehalten. Die notwendigen Stellwege stellen die gesuchte (relative) Höheninformation an jedem Rasterpunkt dar.

Es kommt ein NanoScope STM von Veeco, gesteuert mit einem NanoScope IV Controller zum Einsatz, welches an Luft bei Raumtemperatur arbeitet. Die Messspitzen werden durch gleichzeitiges Reißen und Schneiden eines PtIr-Drahtes hergestellt.

\footnotetext{
${ }^{8}$ Insbesondere war zu untersuchen, ob strukturelle Effekte der sehr dünnen Filme für mechanische Effekte verantwortlich sein können.
} 
AFM: Messprinzip: Die Rasterkraftmikroskopie eignet sich auch für nicht leitende Proben und ist daher noch weiter verbreitet als die Rastertunnelmikroskopie. Dabei wird ein cantilever mit einer scharfen Spitze versehen. Diese rastert die Probe mechanisch ab. Im einfachen contact mode wird die Spitze bis zu einer vorgegeben Verbiegung auf die Probe gedrückt. Beim Rastern wird diese Verbiegung durch Nachregeln der Probenposition mit einem Piezo-Scanner konstant gehalten und die notwendigen Höhenänderungen als Topographie der Probenoberfläche interpretiert.

Ein weiterer verwendeter Bildmodus ist der tapping mode ${ }^{T M}$, bei dem der cantilever zu resonanter Biegeschwingung angeregt wird. Bei Annäherung an die Probe reduziert sich die Amplitude, im einfachsten Fall erklärt durch leichtes Auftreffen. Die gegenüber der freien Schwingung reduzierte Amplitude ist in diesem Modus die nachzuregelnde Stellgröße. Dieser Modus zeichnet sich durch geringere Wechselwirkung mit der Probenoberfläche aus, was teilweise zu deutlich weniger Bildstörungen führt.

Es wird ein MultiMode AFM von Veeco, gesteuert mit einem NanoScope IV Controller, mit verschiedenen Messspitzen verwendet. Messungen im tapping mode werden mit Budget Sensor BS Tap300 Spitzen durchgeführt. Einige für diese Arbeit hergestellte Schichten werden auch für Untersuchungen mittels eines $\operatorname{AFAM}$ (Atomic force acoustic microscopy)-Messaufbaus verwendet. Diese werden von C. Vree und H. Wagner durchgeführt, der Messaufbau ist beschrieben in [55]. Gleichzeitig werden dabei auch konventionelle contact mode-Bilder der Schichten aufgenommen.

Es erwiesen sich generell Siliziumspitzen mit diamantartiger ta-C (tetragonal amophous carbon)-Beschichtung gegenüber reinen Si-Spitzen als sehr vorteilhaft in der Abbildung der PdCuSi-Schichten. 


\section{Kapitel 4}

\section{Ergebnisse}

Dieses Kapitel bescheibt die wesentlichen Messergebnisse der experimentellen Arbeiten. Eine Interpretation und Verknüpfung der Ergebnisse folgt im Kapitel 5. Die gezeigten Ergebnisse stellen eine Auswahl dar, die eine übersichtliche Darstellung ermöglichen. Von den besonders wichtigen Spektroskopieergebnissen der amorphen dünnen Schichten werden jedoch alle Messdurchläufe gezeigt.

Die Messergebnisse werden wie folgt vorgestellt: Zuerst werden Zusammensetzung, Mikrostruktur und Oberflächentopographie der dünnen Schichten, die der eigentliche Untersuchungsgegenstand sind, vorgestellt. Es folgen im Abschnitt 4.2.1 Ergebnisse bezüglich der leeren Paddel und ihrer mechanischen Eigenschaften, da sich diese auch in den Messkurven der dünnen Schichten deutlich abzeichnen. Anschließend werden die Ergebnisse der dicksten Schicht gezeigt. Zu dieser Probe werden anschließend mechanische Messungen unter Raumtemperatur vorgestellt. Ab Abschnitt 4.2.4 sind die Verlustspektren der dünnen Schichten von $50 \mathrm{~nm}$ und dünner aufgezeigt.

\subsection{Charakterisierung der Schichten}

Wie im Kapitel 3.1.2 beschrieben, ist zu jeder Schicht, die auf ein DPO aufgebracht wurde, gleichzeitig eine Referenzschicht auf ein weiteres Substrat aufgedampft worden. Wegen der direkten räumlichen Nähe $(\leq 1 \mathrm{~cm})$ bei der Bedampfung werden die Ergebnisse der Referenzschichten auf die Paddelschichten übertragen. 


\subsubsection{Zusammensetzung der Schichten, EDX}

Die Herstellung der 3-komponentigen Legierung mit drei Elektronenstrahlverdampfern und drei Schwingquarzwaagen gibt durch die Ratenanzeige und angezeigte Schichtdicke der Einzelkomponenten (auf den SQW) zusammen mit der Aufdampfzeit bereits eine gewisse Kontrolle, ob die Schicht die gewünschte Zusammensetzung $\mathrm{Pd}_{77,5} \mathrm{Si}_{16,5} \mathrm{Cu}_{6}$ aufweist oder ob Abweichungen davon vorliegen. Da jedoch der genaue Palladiumanteil sehr stark in die Dichte eingeht und damit in die Berechnung des Schermoduls der Schicht aus der Frequenzverschiebung nach Gleichung (2.27), ist eine Nachkontrolle mittels EDX-Analyse immer notwendig. Nur der dünnste Film wurde durch eine direkt zuvor hergestellte dickere Referenz ${ }^{1}(\approx 50 \mathrm{~nm})$ kontrolliert.

Die Tabelle 4.1 fasst die Ergebnisse der Analysen zusammen.

\begin{tabular}{|c|c|c|c|c|}
\hline Probe & $\begin{array}{c}\text { Nom. Dicke } \\
\text { [nm] }\end{array}$ & $\begin{array}{c}\text { Palladium } \\
\text { [at. \%] }\end{array}$ & $\begin{array}{c}\text { Silizium } \\
\text { [at. \%] }\end{array}$ & $\begin{array}{c}\text { Kupfer } \\
\text { [at. \%] }\end{array}$ \\
\hline DPO_DB_04 & 100 & 73 & 20 & 7 \\
\hline DPO_DB_05 & 200 & 73,5 & 20 & 6,5 \\
\hline DPO_DB_05a & 50 & 74 & 18 & 8 \\
\hline DPO_DB_07 & 50 & 73 & 19,5 & 7,5 \\
\hline DPO_DB_08 & 25 & 72 & 20 & 8 \\
\hline Ref_090204 & 50 & 70 & 21,5 & 8,5 \\
\hline DPO_DB_13(*) & 10 & 64 & 26,5 & 9,5 \\
\hline
\end{tabular}

Tabelle 4.1: Übersicht der EDX-Messungen an den Referenzschichten. Die mit $\left(^{*}\right)$ gekennzeichnete Messung ist durch die zu geringe Schichtdicke evtl. verfälscht.

Die Übersicht zeigt, dass der Palladiumanteil etwas kleiner angezeigt wird als in der Soll-Zusammensetzung, die Streuung in der Serie ist aber gering. Eine Palladiumkonzentration von 70 at.\% scheint kritisch zu sein, Schichten, die mit geringerem Pd-Gehalt hergestellt worden sind stellten sich in der Weitwinkel-Röntgenanalyse als kristallin heraus, auch die mechanischen Eigenschaften solcher Proben deuten auf Kristallinität hin. Für die Probe DPO_DB_13 mit nur $10 \mathrm{~nm}$ Schichtdicke war nicht mehr zu erwarten, dass EDX- und Röntgenanalysen ein deutliches Signal liefern können, daher wurde zuvor eine Referenzschicht von $50 \mathrm{~nm}$ auf ein Substrat abgeschieden. Dessen Zusammensetzung lässt für die dünnste Schicht eine ,richtige“ Zusammensetzung erwarten, da die Palladiumrate anschließend noch um $8 \%$ angehoben wurde. Die EDX-Messung an der dünnsten Referenzprobe selbst ist wesent-

\footnotetext{
${ }^{1}$ Die Referenzprobe wird mit Ref_090204 bezeichnet.
} 
lich stärker fehlerbehaftet als die anderen Messungen, und auch durch den Substratuntergrund evtl. bereits deutlich verfälscht. Das Messergebnis ist daher mit $\left(^{*}\right)$ gekennzeichnet.

Die Röngenquanten einer EDX-Messung an dünnen Schichten stammen, aufgrund der hohen Eindringtiefe des Elektronenstrahls verglichen mit der Filmdicke, überwiegend vom Substrat ${ }^{2}$. In Abbildung 4.1 ist das EDX-Spektrum, gemessen für eine $50 \mathrm{~nm}$ PdCuSi-Schicht (Ref_090204) auf Saphir $\left(\mathrm{Al}_{2} \mathrm{O}_{3}\right)$ vollständig dargestellt. Man erkennt die Dominanz der Ausschläge für Aluminium und Sauerstoff, die dem Substrat zugeordnet werden.

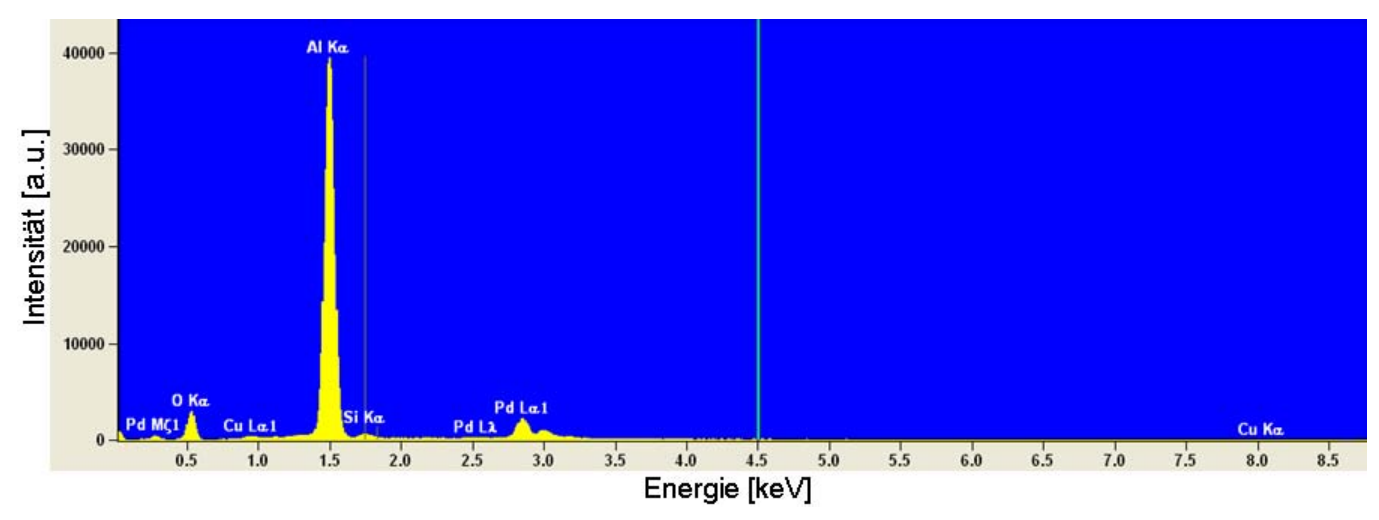

Abbildung 4.1: EDX-Spektrum einer 50 nm PdCuSi-Schicht (Ref_090204) auf Saphir $\left(\mathrm{Al}_{2} \mathrm{O}_{3}\right)$.

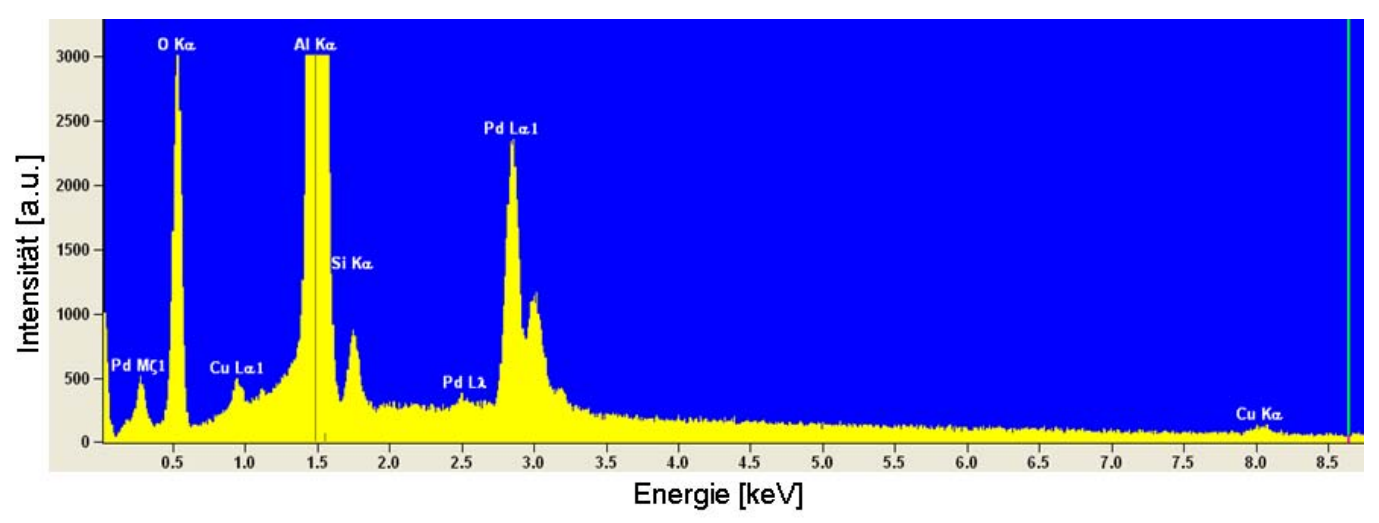

Abbildung 4.2: EDX-Spektrum einer 50 nm PdCuSi-Schicht (Ref_090204) auf Saphir $\left(\mathrm{Al}_{2} \mathrm{O}_{3}\right)$, vergrößerte Darstellung.

\footnotetext{
${ }^{2}$ Der Versuch diese Problematik durch oberflächensensitive XPS-Messungen zu überwinden erwies sich als nicht hilfreich, vermutlich aufgrund von Oberflächenoxidation.
} 
Eine Vergrößerung desselben Spektrums zeigt Abbildung 4.2. In dieser Darstellung erkennt man noch deutliche isolierte Ausschläge der gesuchten Elemente im Spektrum. Diese Abgrenzung wird bei noch dünneren Filmen unklarer.

\subsubsection{Strukturelle Analyse mit XRD}

\section{Weitwinkel-Röntgenstreuung:}

Die strukturelle Analyse erfolgte mit Weitwinkel-Röntgenstreuung mit dem Ziel die Amorphizität der Schichten zu beurteilen. Bei hinreichend langer Messzeit liefert die Streuung an den dünnen amorphen Schichten noch ausreichend Intensität, um diese Frage zu beantworten. Die Vermessung erfolgte an allen Referenzschichten. Beispielhaft ist in Abbildung 4.3 das WeitwinkelRöntgenspektrum der Probe DPO_DB_08 (25 nm nominelle Schichtdicke) gezeigt, da diese Probe die in dieser Serie dünnste Schicht mit ausreichender Intensität darstellt. Man erkennt trotz einer Verkippung der Probe einige scharfe Reflexe von dem Saphir-Substrat, jedoch ist der breite Peak davon nicht überdeckt.

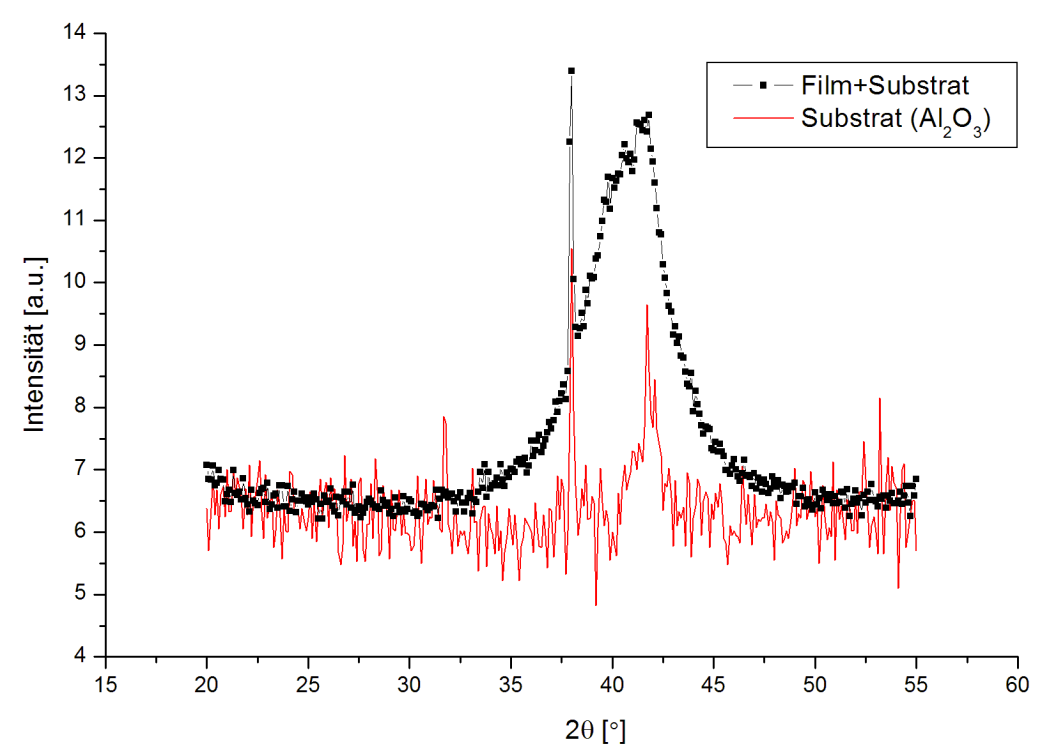

Abbildung 4.3: Weitwinkel-Röntgenspektrum der Probe DPO_DB_08 $(25 \mathrm{~nm} \mathrm{PdCuSi})$ auf Saphir. Die rote Linie entspricht der Messung eines leeren Substrates bei gleichen Einstellungen von Blenden und Probenverkippung.

Für die noch dünnere $10 \mathrm{~nm}$ dicke Schicht ist ein solcher breiter Peak kaum 
noch zu erkennen, es fehlen jedoch scharfe Peaks, die auf eine kristalline Struktur hinweisen. Eine Abbildung dieser Messung findet sich im Anhang A.1. Die Weitwinkel-Röntgenspektren der weiteren hier verwendeten Proben werden nicht gezeigt, sie weisen alle ein breites Maximum bei etwa $2 \Theta=40,6^{\circ}$ auf.

\section{Kleinwinkel-Röntgenstreuung:}

Die Kleinwinkel-Röntgenmessungen dienen der Kontrolle der Schichtherstellung hauptsächlich in Bezug auf die Schichtdicke. Die Messungen wurden stets direkt nach dem Ausschleusen der Proben aus der UHV-Anlage durchgeführt, um Oxidationseffekte zu vermeiden. Die genaue Schichtdicke wird zur Berechnung des Schermoduls der Schicht auf dem DPO aus der gemessenen Frequenzverschiebung benötigt. Als Nebenprodukt enthalten die Kleinwinkel-Röntgenspektren auch eine Information über die Rauigkeit der Schichtoberfläche. Dieser Parameter ist jedoch auf diese Weise nicht so genau zu bestimmen, wie z. B. über Rastersondentechniken, da der Verlauf über eine Vielzahl weiterer Parameter angepasst wird. Außerdem können die so ermittelten Rauigkeiten nicht direkt auf die Schichten auf den DPOs übertragen werden, da auf diesen der PdCuSi-Film auf einer Ta-Schicht aufwächst. Die Tabelle 4.2 stellt alle Ergebnisse der hier verwendeten Schichten tabellarisch dar. Eine typische Kleinwinkel-Röntgenmessung mit Anpassung nach dem Parrat-Algorithmus [63] ist im Anhang A.1 abgebildet. Die Probe mit nominell $200 \mathrm{~nm}$ Schichtdicke (DPO_DB_05) konnte auf Grund der Dicke nicht vermessen werden.

\begin{tabular}{|c|c|c|c|}
\hline Probe & $\begin{array}{c}\text { Nom. Dicke } \\
{[\mathrm{nm}]}\end{array}$ & $\begin{array}{c}\text { Beste Anpassung } \\
{[\mathrm{nm}]}\end{array}$ & $\begin{array}{c}\text { Rauigkeit } \sigma \\
{[\AA]}\end{array}$ \\
\hline DPO_DB_04 & 100 & 93 & 9,5 \\
\hline DPO_DB_05a & 50 & 52 & 6,5 \\
\hline DPO_DB_07 & 50 & 55 & 6,5 \\
\hline DPO_DB_08 & 25 & 27,5 & 5 \\
\hline Ref_090204 & 50 & 52,5 & 7 \\
\hline DPO_DB_13 $\left(^{*}\right)$ & 10 & 10,5 & 6 \\
\hline
\end{tabular}

Tabelle 4.2: Tabellarische Darstellung der Anpassungsparameter von ParratReflexionskurven zu den gemessenen Kleinwinkel-Röntgenspektren. Die Angabe der Rauigkeit ist mit einer geschätzten Unsicherheit von $\pm 1 \AA$ eher ein qualiatatives Vergleichskriterium.

Die Bestimmungen der Schichtdicken der Proben bestätigen den Zielvorgaben entsprechend abgelaufene Beschichtungen. Nur zwei Proben weichen laut Röntgenanalyse um mehr als 2-3 nm von der Vorgabe ab. Dabei ist zu be- 
merken, dass das Beschichten durch manuelles Betätigen des Shutters gestartet und beendet wird. Die Unsicherheit der Beschichtungsdauer beträgt dadurch geschätzte $2 \mathrm{~s}$, was bei einer Aufdampfrate von $\approx 13 \AA / \mathrm{s}$ die typischen Schichtdickenabweichungen bereits ohne weitere Messfehler erklären könnte.

Die angepassten Rauigkeiten zeigen keine signifikanten Unterschiede zwischen den Schichten, mit Ausnahme der dicksten so gemessenen Probe.

\subsubsection{Oberflächentopographien}

Einige Schichten wurden auch auf ihre Oberflächenbeschaffenheit mittels STM, AFM und REM untersucht. Dabei lag der Fokus auf der Frage, ob die Schichten homogen geschlossen und glatt vorliegen oder ob topographische Änderungen bei bestimmten Schichtdicken auftreten, die bei der Interpretation der mechanischen Messungen zu berücksichtigen sind. Zudem sollten auch die Substratrauigkeiten überprüft werden.

\section{Oberfläche der Tantalschicht:}

Die mechanisch untersuchten Schichten wurden alle ${ }^{3}$ auf eine $50 \mathrm{~nm}$ dicke Tantalschicht aufgebracht. Eine solche gesputterte Tantalschicht wächst auf Silizium glatt auf [68], dennoch wurde dies für die hier verwendeten Sputterparameter noch einmal überprüft. Eine STM-Messung einer Tantal Schicht auf Silizium zeigt eine glatte Oberfläche bestehend aus kleinen Körnern von 5-10 nm Durchmesser (Abb. 4.4). Das dargestellte Bild ist mit einem Line-Fit jeder Bildzeile begradigt worden. Einen deutlich größeren Bereich der Oberfläche zeigt eine REM-Aufnahme in Abbildung 4.5. Auch in dieser Darstellung scheint die Oberfläche nur aus gleichmäßig kleinen Körnern zu bestehen.

\section{Kristallisierte PdCuSi-Schichten:}

In [6] erwiesen sich kristallisierte ZrAlCu-Schichten als brauchbare Substrate, um weitere amorphe Filme aufzubringen. Dieses Verfahren bringt einen erheblichen Zeitvorteil, gegenüber der Alternative für jeden Film ein neues Doppelpaddel in die UHV-Anlage einzubauen und zu charakterisieren. An dieser Stelle wird die Rauigkeit eines kristallisierten PdCuSi-Filmes gezeigt. Es wurde ein Doppelpaddel-Oszillator (DPO_DB_07) mit einem im Vakuum kristallisierten Film ausgebaut und mittels STM untersucht. Ein solches Bild zeigt Abbildung 4.6. Der mit $50 \mathrm{~nm}$ Dicke amorph hergestellte Film

\footnotetext{
${ }^{3}$ Das Paddel DPO_DB_04 wurde ohne eine Tantal Barriere beschichtet und gemessen. Die dabei gewonnen Spektren werden nicht mit den weiteren Ergebnissen verglichen (siehe Abschnitt 4.2.5).
} 


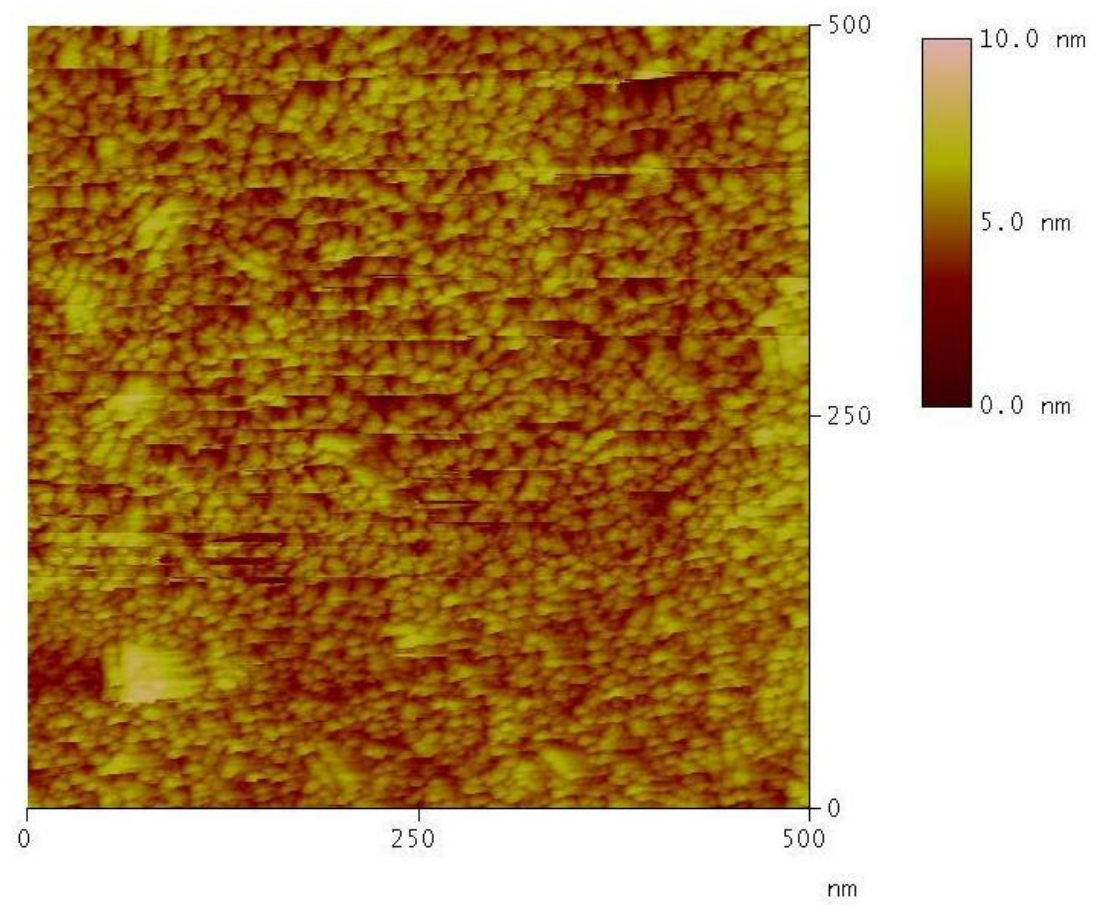

Abbildung 4.4: STM-Bild einer $50 \mathrm{~nm}$ Tantal Schicht auf Silizium. Man erkennt eine feinkörnige Oberfläche. Die RMS-Rauigkeit in diesem Bild beträgt etwa $1,3 \mathrm{~nm}$.

erweist sich nach der Kristallisation aufgerissen und nicht mehr zusammenhängend. Dazwischen erkennt man tiefe „Gräben“. Es ergeben sich typische „peak-to-valley"-Werte von bis zu $80 \mathrm{~nm}$.

\section{Topographie dünner amorpher PdCuSi-Schichten:}

Die Topographie amorpher PdCuSi-Schichten an den Referenzschichten der dünnsten Proben in dieser Arbeit ist ebenfalls überprüft worden. Um vergleichbare Resultate ${ }^{4} \mathrm{zu}$ erhalten, sind diese Topographien mit dem im Abschnitt 3.4.4 beschriebenen AFM im tapping mode $e^{T M}$ aufgenommen.

Die Abbildung 4.7 zeigt eine AFM-Aufnahme der Referenzschicht des dünnsten mechanisch vermessenen Films. Darauf erkennt man keine Hinweise auf einen unzusammenhängenden Film oder große Inseln. Die kleinen, im Vergleich zur Filmtopographie hohen, Körner sind typisch für unbehandelte Silizumwafer [69].

\footnotetext{
${ }^{4}$ Die AFM-Spitzen einer Charge variieren weniger als handgezogene STM-Spitzen.
} 


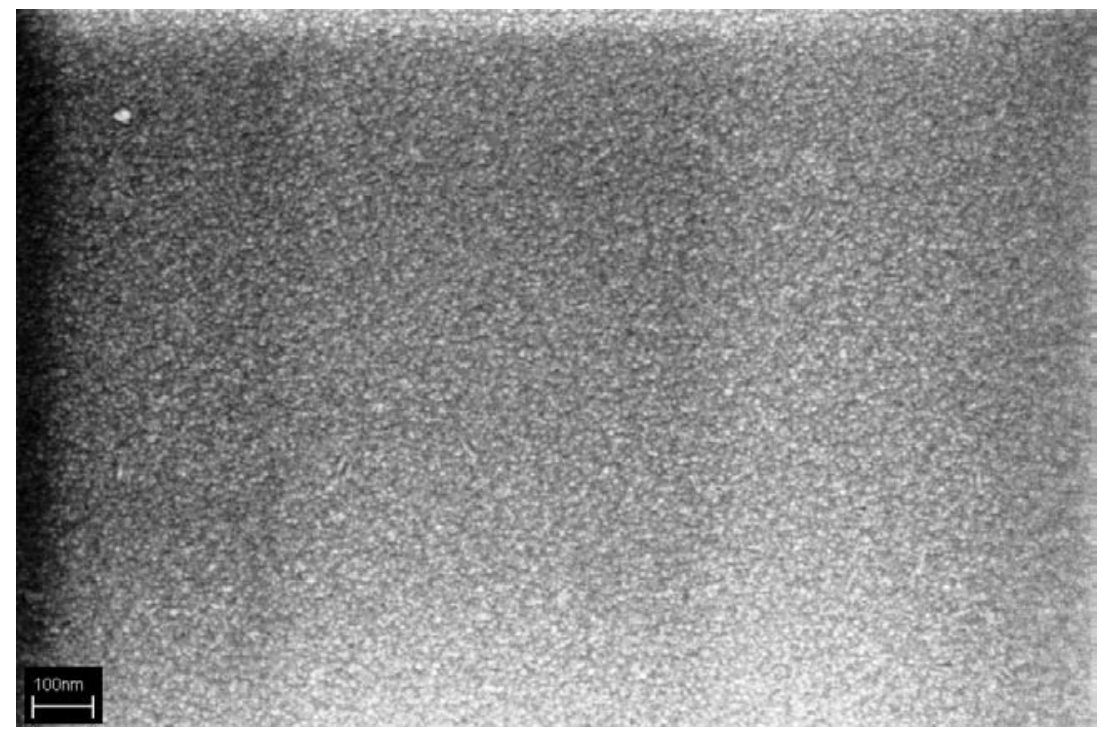

Abbildung 4.5: REM-Aufnahme einer $50 \mathrm{~nm}$ Tantalschicht auf Silizium. Die Oberfläche erscheint gleichmäßig feinkörnig.

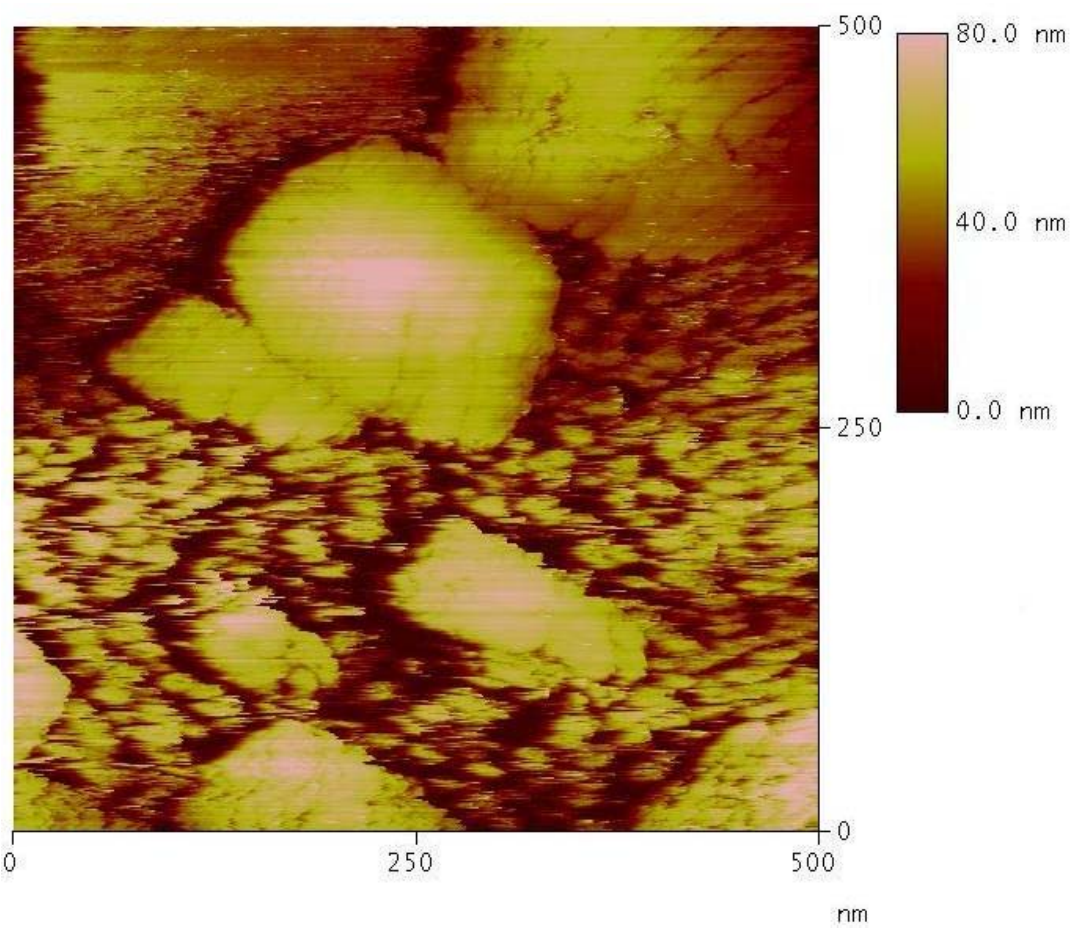

Abbildung 4.6: STM-Bild eines kristallisierten PdCuSi-Films mit 50 nm Dicke. 


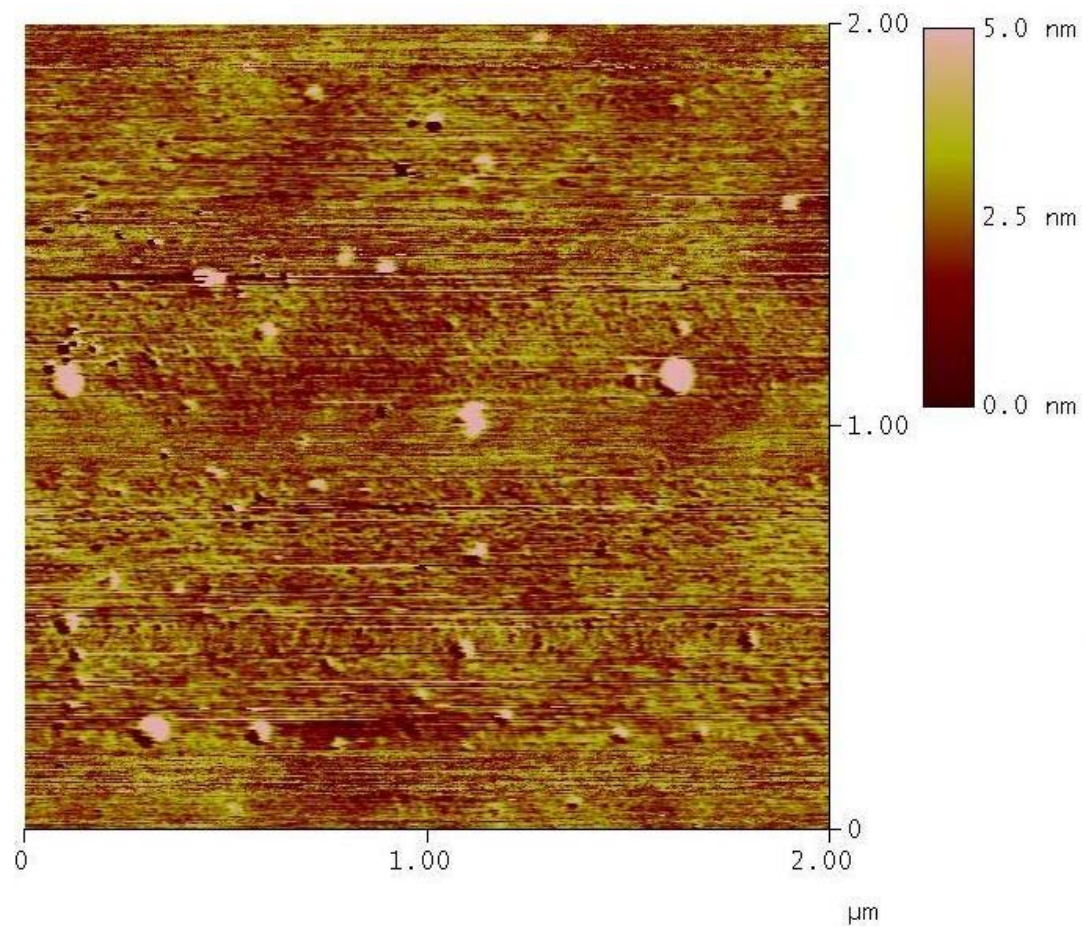

Abbildung 4.7: AFM-Bild einer $10 \mathrm{~nm}$ PdCuSi-Schicht auf einem Silizium Referenzsubstrat. 


\subsection{Mechanische Messungen}

\subsubsection{Unbeschichtete Doppelpaddel-Oszillatoren}

Die Ergebnisse zu den mechanischen Messungen beginnen mit den unbeschichteten Oszillatoren, da deren Eigenschaften die gemessenen Spektren stets dominieren und die zu vermessenden Schichten immer nur geringe Änderungen dieser Eigenschaften verursachen.

Zunächst wird daran erinnert, dass die Paddeltemperatur indirekt an der Klemmung gemessen wird, deren Temperatur, wie in Abschnitt 3.1.1 beschrieben, mit der Paddelkopftemperatur in bekannter Weise zusammenhängt. Daraus resultiert der hystereseartige Verlauf der Oszillatordämpfung als Funktion der Klemmblocktemperatur beim Heizen und Kühlen in Form von Rampen. Ein solcher Verlauf ist in Abbildung $4.8 \mathrm{zu}$ erkennen: Beim Heizen steigt die Dämpfung zunächst langsam und zu höherer Temperatur immer steiler an. Beim Erreichen der höchsten Temperatur von $350{ }^{\circ} \mathrm{C}$ wird das Paddel für 30 Minuten gehalten, anschließend wird es mit konstanter Rate wieder abgekühlt. Die Abweichung des Kühlverlaufes kann mit der Temperaturverschiebung von Paddelkopftemperatur zu Blocktemperatur bei Heizen und Kühlen erklärt werden: Mit der Frequenzmessung der unbe-

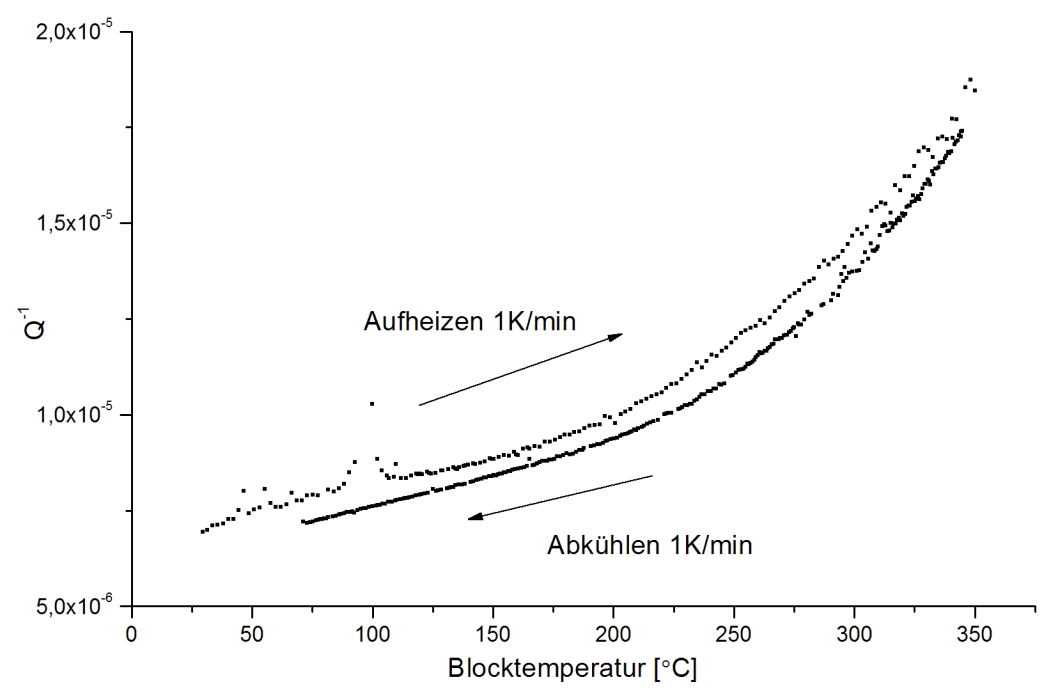

Abbildung 4.8: Leermessung von DPO_DB_05 mit Kühlen und Heizen in einem Durchlauf. Die Abweichungen von Heizen und Kühlen sind durch die Temperaturmessung bedingt. 
schichteten Oszillatoren steht ein sehr präzises Maß für die Paddelkopftemperatur zur Verfügung. Nimmt man an, dass bei gleicher Resonanzfrequenz bei Heizen und Kühlen die gleiche Paddelkopftemperatur vorliegt, so können die Abkühlkurven über das Kriterium der gleichen Resonanzfrequenz mit den Aufheizkurven zur Deckung gebracht werden. Dies ist in Abbildung 4.9 demonstriert: Die Temperaturskala wird von der Aufheizkurve übernom-

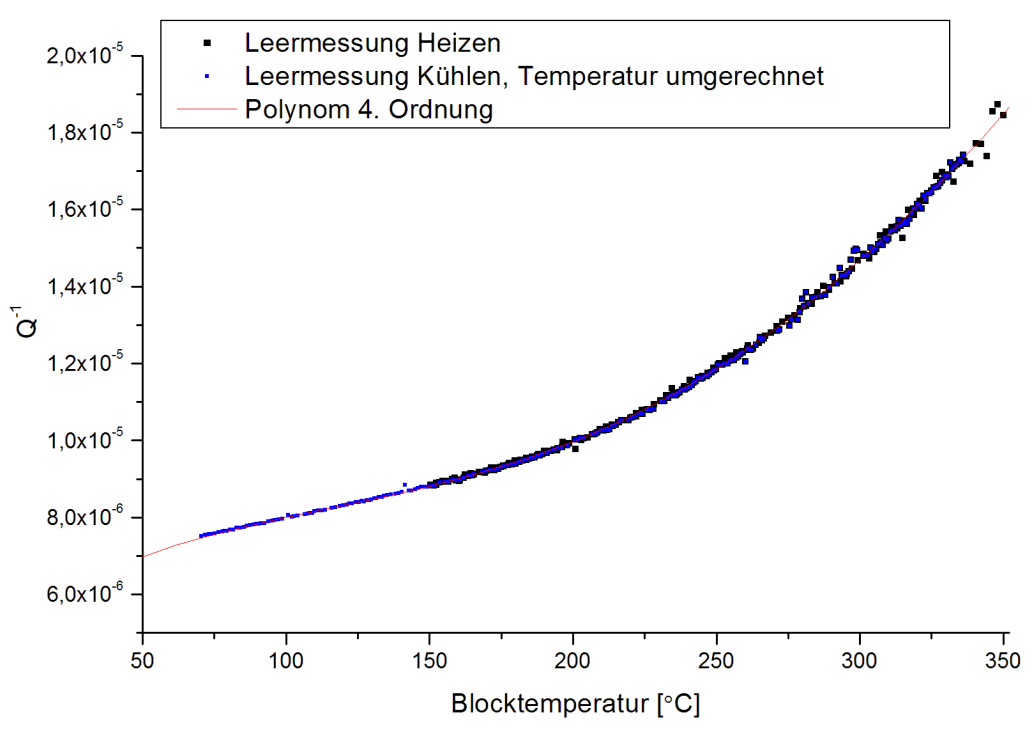

Abbildung 4.9: Die beim Heizen und Kühlen gemessene Dämpfung des leeren Doppelpaddel-Oszillators kann über die Zuordnung der Resonanzfrequenz mit einem einzigen Polynom angepasst werden. Der Temperaturbereich unter $150{ }^{\circ} \mathrm{C}$ aus der Heizmessung wurde auf Grund der in Abbildung 4.8 erkennbaren Störung ausgelassen.

men. Die gemessenen Dämpfungswerte der Abkühlkurve sind mit der, bei jeder Dämpfungsmessung gleichzeitig gemessenen, Resonanzfrequenz nun der Blocktemperatur der Aufheizmessung zugeordnet worden, bei der die gleiche Resonanzfrequenz auftritt. Das Ergebnis rechtfertigt die Annahme deutlich. Alle in Abbildung 4.9 eingetragenen Messpunkte können mit dem rot eingetragenem Polynom gemeinsam beschrieben werden.

Über die Resonanzfrequenz können verschiedene Leermessungen auf Konsistenz zueinander geprüft werden. Eine solche Übersicht ist in Abbildung 4.10 gezeigt. Der gemessene Temperaturbereich setzt sich aus reinen Kühlmessungen mit flüssigem Stickstoff und Messungen unter Verwendung der Lampenheizer zusammen. Dennoch gelingt die Zusammenfügung zu einem Verlauf. Der dargestellte Temperaturbereich erstreckt sich von -75 bis $350{ }^{\circ} \mathrm{C}$. 
Die Dämpfung steigt mit der Temperatur zunächst stark an, ansschließend flacht der Anstieg ab. Ab $\approx 160{ }^{\circ} \mathrm{C}$ wird der Anstieg wieder kontinuierlich steiler. Es existiert ein scharf begrenztes Maximum in der Dämpfung nahe der Raumtemperatur. Dämpfungsanomalien wie diese werden in Kapitel 5.2 diskutiert.

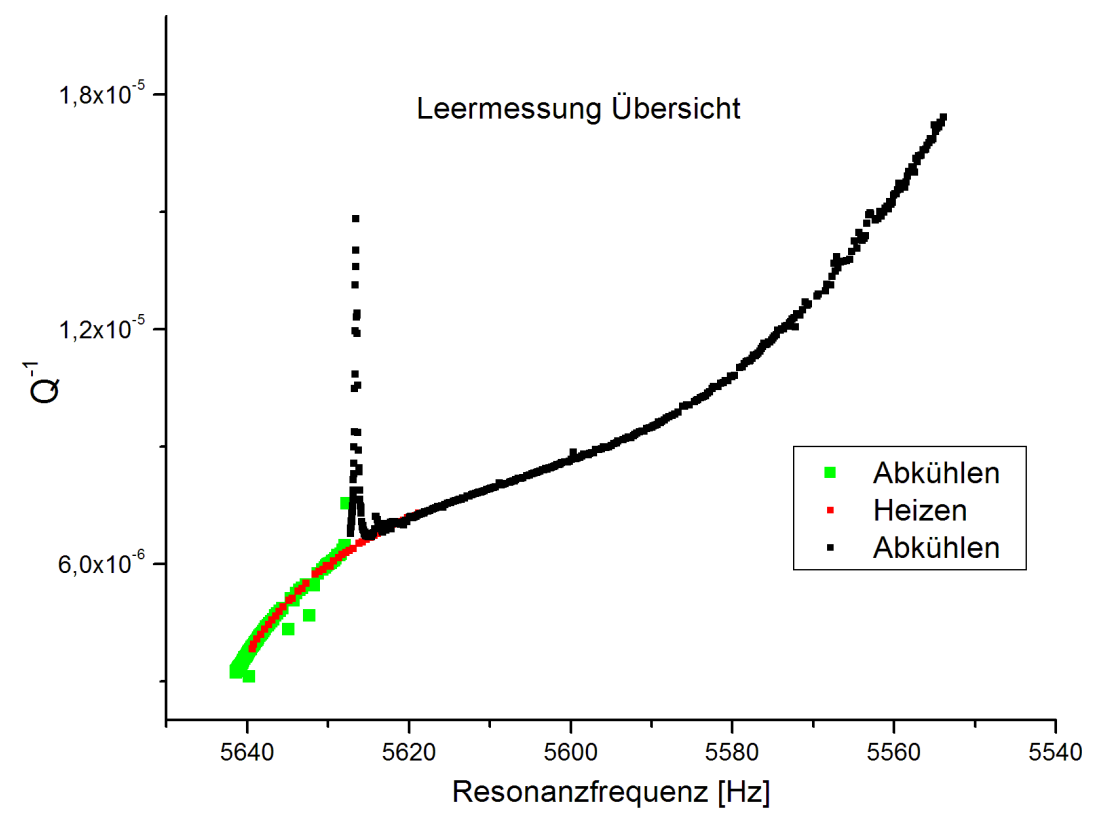

Abbildung 4.10: Zusammenstellung verschiedener Dämpfungsmessungen des unbeschichteten Paddels DPO_DB_05. Gegenüber der Resonanzfrequenz aufgetragen ergibt sich ein einheitlicher Verlauf. Der entsprechende Temperaturbereich erstreckt sich von -75 bis $350{ }^{\circ} \mathrm{C}$ Blocktemperatur. Der scharfe Peak liegt nahe bei Raumtemperatur.

Die Messung der Resonanz- bzw. Eigenfrequenz der AS2-Mode als Funktion der Temperatur führt zu einem fast linearen Verlauf, wie er in Abbildung 4.11 dargestellt ist. Eine gute Anpassung des Verlaufs erfordert zusätzlich einen geringen quadratischen Beitrag ${ }^{5}$. Die hier gemessene Resonanzfrequenz sinkt von $5626 \mathrm{~Hz}$ bei RT auf $5551 \mathrm{~Hz}$ bei $350{ }^{\circ} \mathrm{C}$. Damit sinkt die Resonanzfrequenz um etwas mehr als $100 \mathrm{~Hz}(\approx 2 \%)$.

In Abschnitt 2.4.4 wurde der Einfluss von Kontaminationen auf die Dämpfungsmessung angesprochen. Es soll hier eine Demonstration der hohen Empfindlichkeit des DPOs auf solche Kontaminationen gezeigt werden. Für das

\footnotetext{
${ }^{5}$ Mit der Anpassung mit $f_{A S 2}(T)=f_{0}+a T+b T^{2}$ beträgt das Verhältnis der Koeffizienten $b / a \approx 2 * 10^{-4}{ }^{\circ} C^{-1}$.
} 


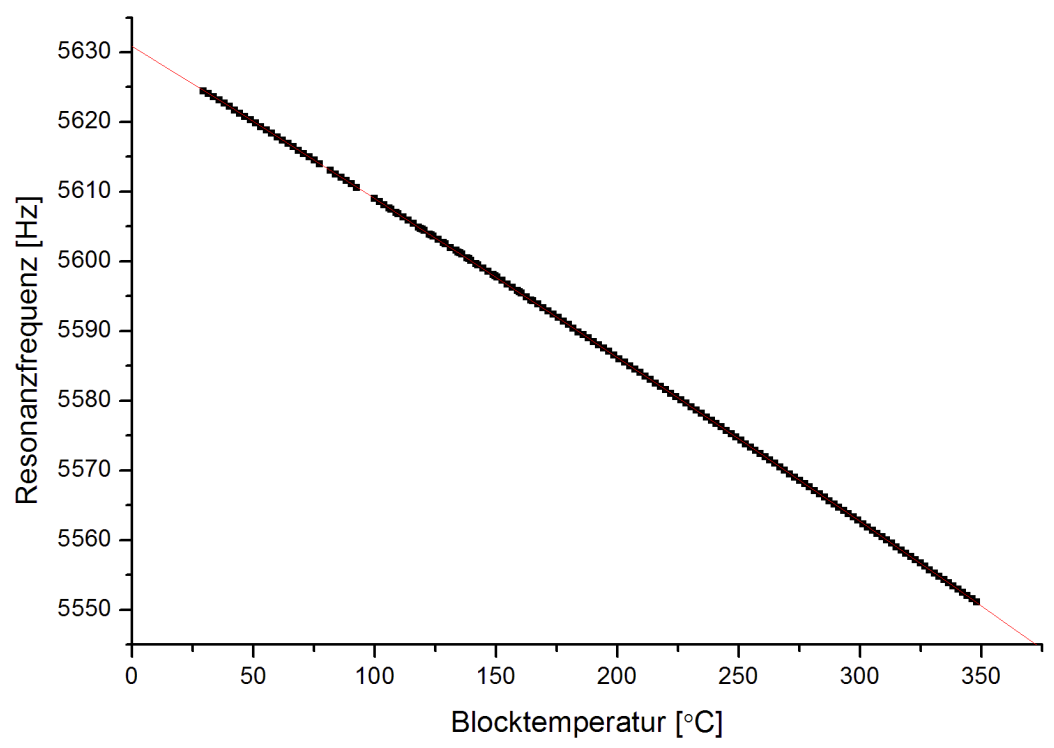

Abbildung 4.11: Temperaturverlauf der AS2-Mode für das leere Paddel DPO_DB_05. Die Anpassung entspricht einem Polynom 2. Ordnung.

Doppelpaddel DPO_DB_02 wurde der Dämpfungsverlauf nach dem Einbau in die UHV-Anlage bei einem Druck von $3^{*} 10^{-7}$ mbar gemessen. Die UHVAnlage wurde anschließend ausgeheizt (wie in Abschnitt 3.1.2 beschrieben) und der Verlauf erneut bei einem Druck von $5^{*} 10^{-10}$ mbar gemessen. Den Vergleich zeigt die Abbildung 4.12. Die Dämpfung des noch nicht ausgeheizten Paddels ist stark erhöht und weicht auch oberhalb von $100{ }^{\circ} \mathrm{C}$ stark von dem sauberen Paddel ab. Das Signal einer solchen Kontaminationsschicht übersteigt die erwartete Dämpfung dünner amorpher Filme deutlich. Alle quantitativ auszuwertenden Messungen werden daher nach gründlichem Ausheizen der Paddel mit den Lampenheizern und der gesamten UHV-Kammer durchgeführt.

\subsubsection{Mechanische Analyse einer dicken PdCuSi-Schicht}

Die an dieser Stelle gezeigten Ergebnisse dienen als bulk-artige Referenz zu den später vorgestellten Messungen an dünnen Schichten unter $100 \mathrm{~nm}$. Als massive Schicht wurde eine Schichtdicke von $200 \mathrm{~nm}$ gewählt, da für diese Dicke keine Dimensionseffekte erwartet werden. Diese Spektren werden in der Diskussion mit früheren Ergebnissen an PdCuSi-Bändern von $\approx 40 \mu \mathrm{m}$ Dicke verglichen (Kap. 5.3.1). 


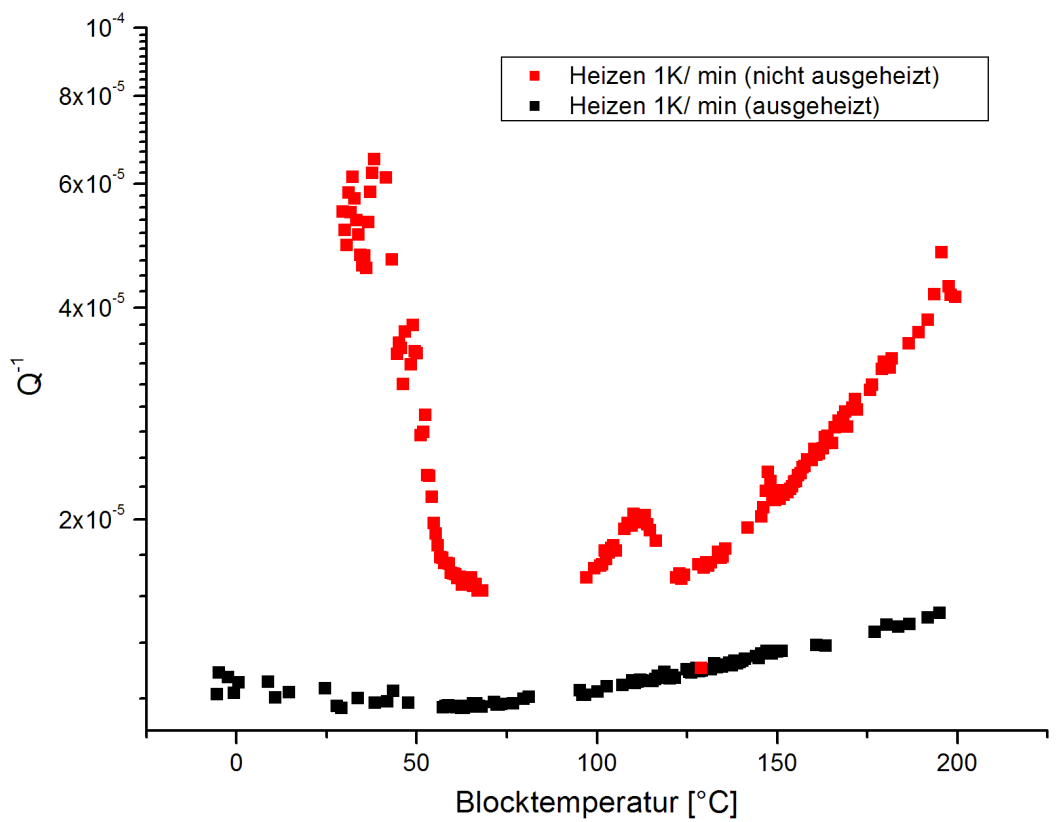

Abbildung 4.12: Vergleichsmessung, die den Einfluss von Kontaminationen vor dem Ausheizen zeigt. Die Dämpfung des ,leeren“ Paddels ist vor dem Ausheizen stark erhöht.

\section{Dämpfungsverlauf:}

Zuerst wird der Verlauf des inversen Qualitätsfaktors des DPOs mit der $200 \mathrm{~nm}$ PdCuSi-Schicht vergleichend mit dem leeren Paddel in Abbildung 4.13 aufgezeigt ${ }^{6}$.

Mit der dicken Schicht beginnt der Verlauf mit einer, gegenüber dem leeren Paddel, deutlich erhöhten Dämpfung, welche sich dieser bis etwa $440 \mathrm{~K}$ wieder annähert. Anschließend steigt die Dämpfung kontinuierlich an, bis sie bei ca. $640 \mathrm{~K}$ steil einbricht und danach wieder ansteigt. Der Verlauf wird noch deutlicher, wenn man, wie in Abbildung 4.14, nur die Differenzdämpfung $\Delta Q^{-1}$ aufträgt.

\section{Frequenzverschiebung:}

Veränderungen im Schermodul der Schicht äußern sich nach Gleichung (2.27) linear in der Frequenzverschiebung. Die Gegenüberstellung der Resonanzfrequenzen des Paddels mit der Schicht zum leeren Paddel ist in Abbildung 4.15 gezeigt. Darin erkennt man über den gesamten Temperaturbereich eine

\footnotetext{
${ }^{6}$ Für Temperaturen nahe Raumtemperatur sind sowohl im Spektrum des leeren Paddels als auch bei dem beschichteten Paddel scharfe Peaks zu beobachten, die nicht der Schicht zugeordnet werden.
} 


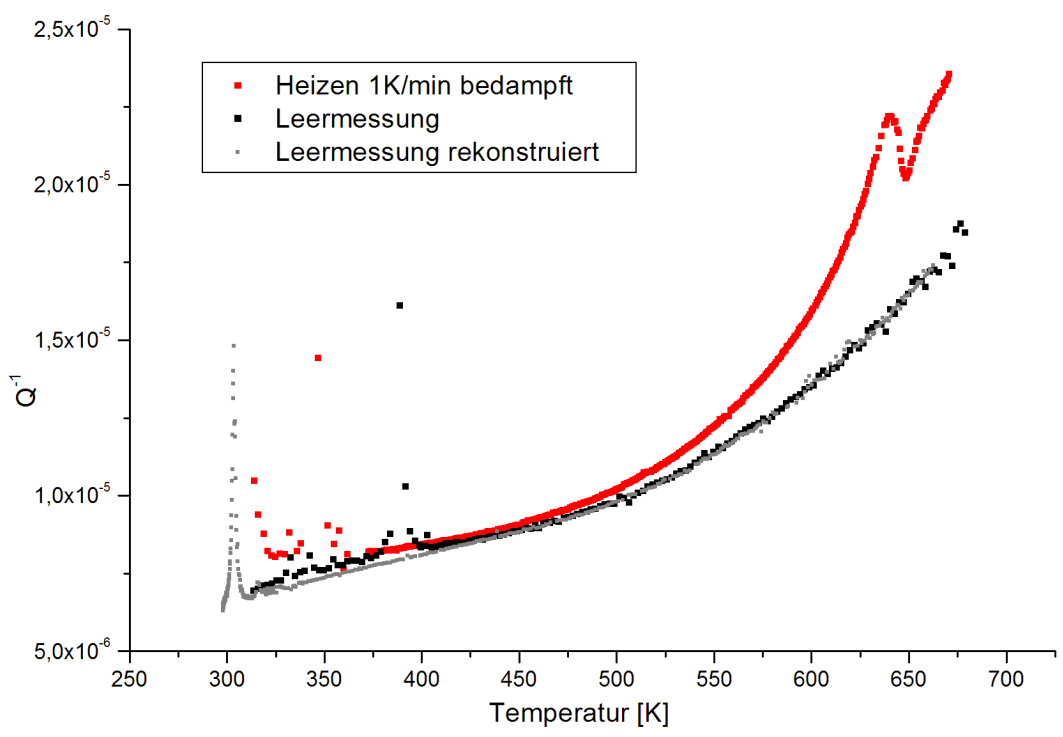

Abbildung 4.13: Verlauf der Dämpfung des DPOs mit einer 200 nm PdCuSiSchicht im Vergleich zu dem leeren Paddel. Die grau dargestellte, aus einer Abkühlmessung bestimmte Leeermessung, entspricht der in Abbildung 4.9 blau dargestellten Messung.

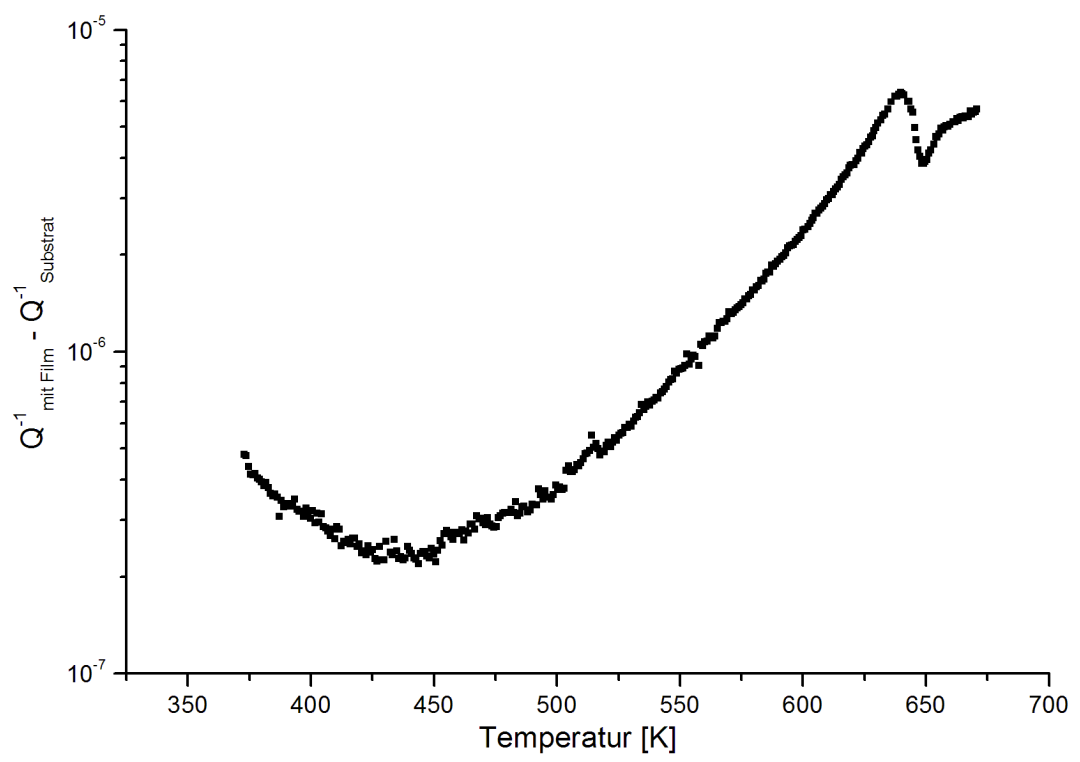

Abbildung 4.14: Dämpfungssignal der 200 nm PdCuSi-Schicht. Die Dämpfung der frisch hergestellten Schicht nimmt zunächst ab, um über einen breiten Temperaturbereich wieder anzusteigen. Bei etwa $640 \mathrm{~K}$ nimmt diese stark ab und steigt danach wieder. 
Absenkung der Resonanzfrequenz durch den Film. Die große Variation der Resonanzfrequenz durch den Temperatureffekt des leeren Paddels führt hier jedoch zu einer Auftragung, in der Änderungen in der Frequenzfrequenzverschiebung nur noch schwer auszumachen sind. Daher werden in Abbildung 4.16 nur die Frequenzabweichungen nach dem Beschichtungsprozess aufgetragen. Sie werden bestimmt, indem die Leermessung, wie in der Abbildung 4.11 dargestellt, polynomial angepasst und die Differenz zu den mit Film gemessenen Frequenzwerten jeweils berechnet wird.

Die Frequenzabsenkung von anfänglich $5 \mathrm{~Hz}$ sinkt beim Heizen zunächst fast linear auf nur noch $4,5 \mathrm{~Hz}$ bei $440 \mathrm{~K}$. Danach erhöht sich die Frequenzabsenkung über ein breites Temperaturintervall kontinuierlich. Bei etwa $625 \mathrm{~K}$ erkennt man ein Einmünden in einen steileren Verlauf, kurz darauf einen gegenteiligen Effekt bei $660 \mathrm{~K}$. Ab $665 \mathrm{~K}$ nimmt die Frequenzabweichung wieder zu.

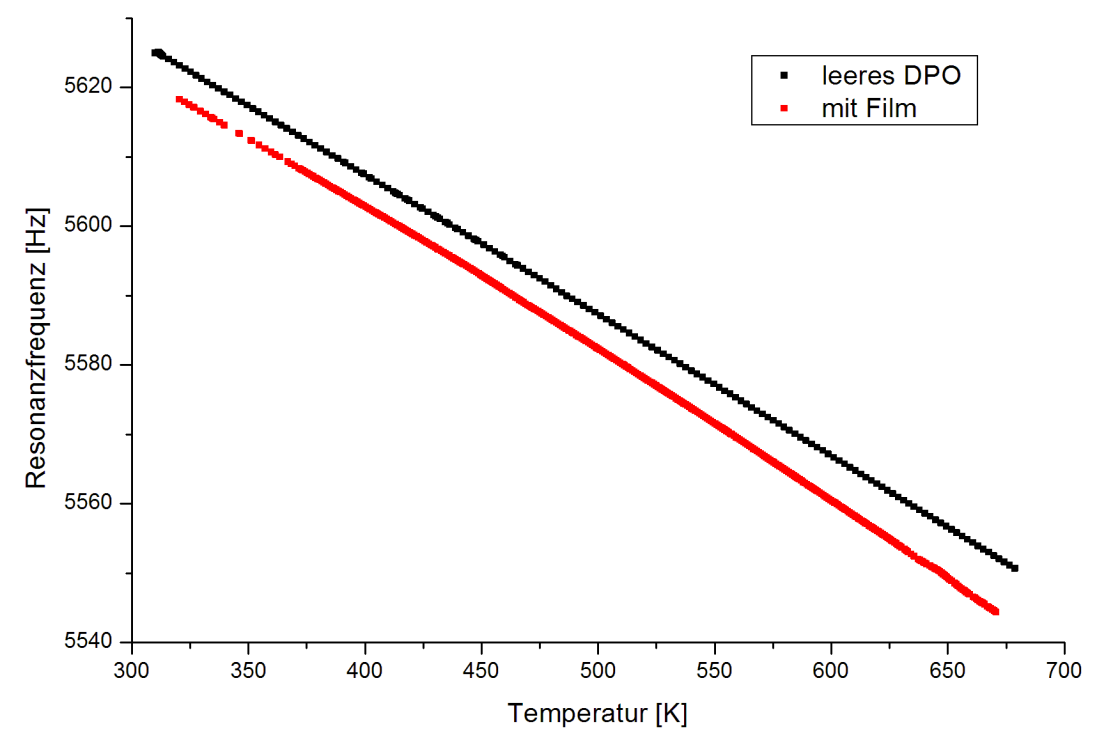

Abbildung 4.15: Verlauf der Resonanzfrequenz über der Temperatur von DPO_DB_05. Dargestellt ist der Verlauf der Frequenz sowohl ohne Film (schwarze Symbole) als auch für den ersten Aufheizvorgang mit dem 200 nm PdCuSi-Film (rote Quadrate). Durch den großen Einfluss der Temperatur auf das Paddel sind Variationen in der Frequenzverschiebung schwer zu erkennen. Dennoch ist der Einfluss des Films insgesamt deutlich. 


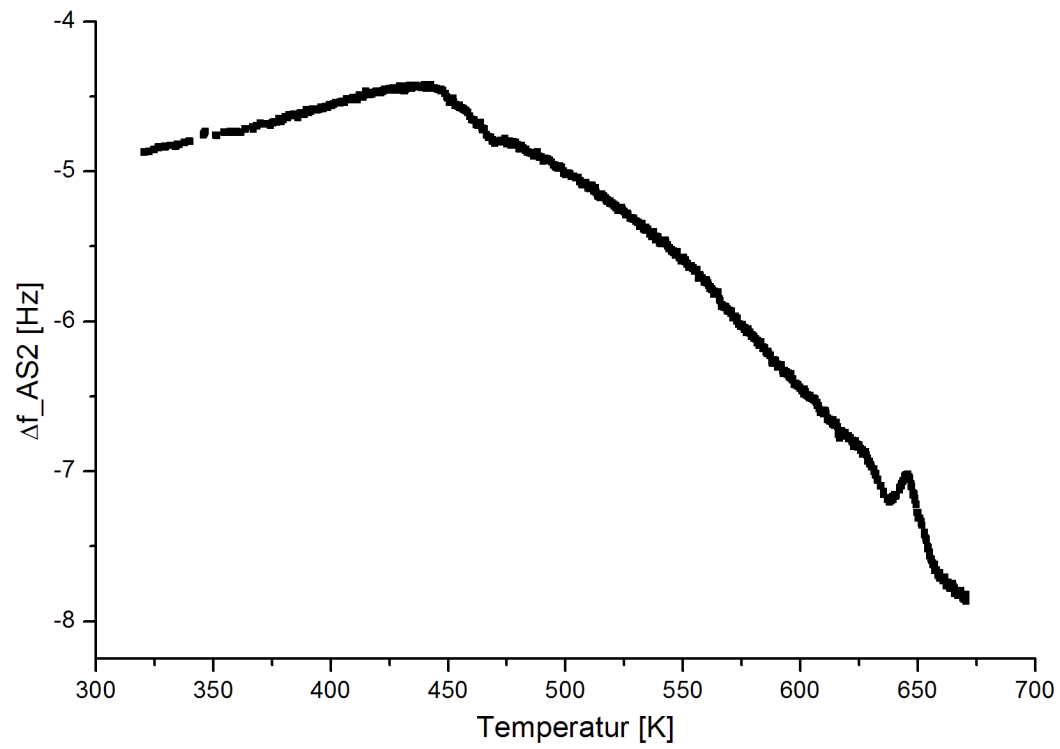

Abbildung 4.16: Frequenzverschiebung durch den 200 nm PdCuSi-Film. In dieser Darstellung besagt Gleichung (2.27), dass ein Absinken der Frequenz einer Reduzierung des Schermoduls der Schicht entspricht, solange alle übrigen Parameter konstant bleiben.

\section{Komplexer Schermodul der Schicht:}

Die Messungen für Frequenzverschiebung und Dämpfungsänderung durch die Schicht können am Besten bewertet werden, wenn sie in einer Auftragung gegenübergestellt sind. Dies soll hier durch eine Auftragung der beiden Komponenten des komplexen Schermoduls der Schicht realisiert werden. Diese Anteile werden, wie in Kapitel 2.4.3 beschrieben, berechnet. Das Ergebnis ist in Abbildung 4.17 gezeigt. Der Verlauf der Anteile entspricht qualitativ den Graphen in den Abbildungen 4.14 und 4.16. 


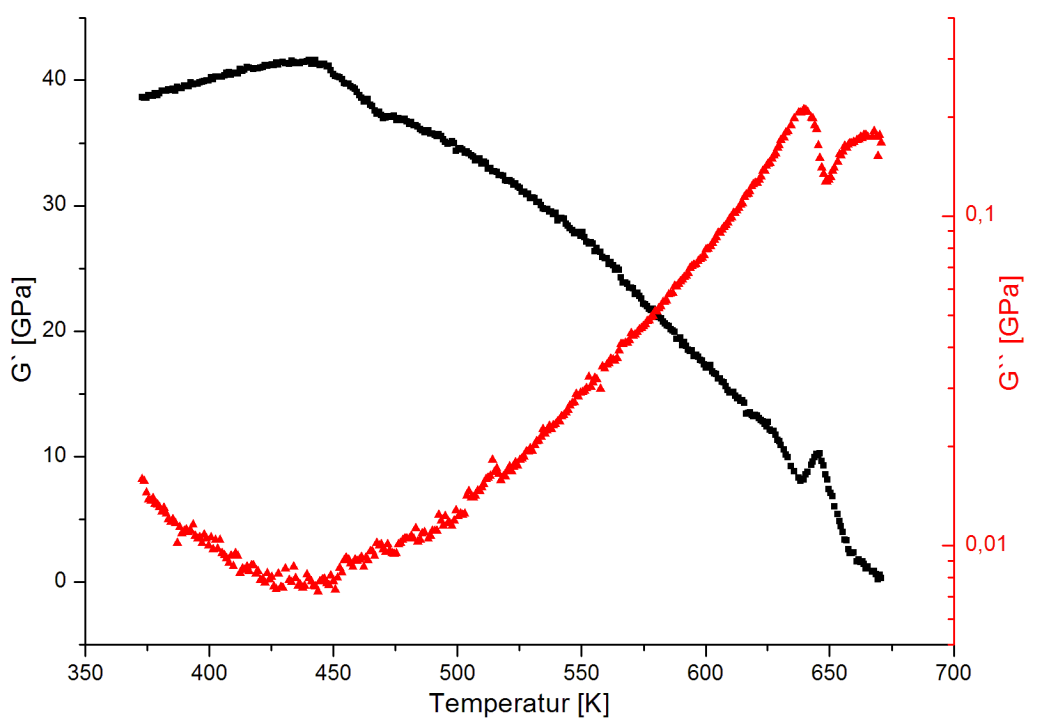

Abbildung 4.17: Berechneter komplexer Schermodul der 200 nm PdCuSiSchicht. Deutlich ist zu erkennen, dass Anstiege in $G^{\prime}$ mit einem Absinken in $G^{\prime \prime}$ und umgekehrt einhergehen.

\subsubsection{Mechanische Messungen unter Raumtemperatur}

In dieser Arbeit wurde auch der Schritt unternommen, den Temperaturbereich des Hochtemperaturmesseinsatzes mit einer Kühlmöglichkeit zu erweitern. Die Ergebnisse der Messungen an amorphen Schichten beim Kühlen mit flüssigem Stickstoff und beim Gegenheizen mit fester Rate bis über RT werden hier exemplarisch gezeigt. Dabei wird besonders auf die dickste Schicht eingegangen, da für die dünneren Schichten das eigentliche Differenzsignal nicht immer sicher von anderen Einflüssen der Messung unterschieden werden kann. Die Abbildung 4.18 zeigt den Verlauf der Dämpfung von DPO_DB_05 mit und ohne amorphe Schicht, in diesem Fall $200 \mathrm{~nm}$. Die Dämpfung des leeren Paddels nimmt in dem dargestellten Temperaturbereich stark ab, der Verlauf der Dämpfung des Paddels mit Schicht folgt diesem Verlauf. Bereits in dieser Darstellung erkennt man eine Abnahme der Differenzdämpfung, die Schicht liefert also einen abnehmenden Beitrag.

In Abbildung 4.19 ist nur die Differenzdämpfung als Funktion der Temperatur für diesen Kühldurchlauf dargestellt. Man erkennt eine kontinuierliche Abnahme der Schichtdämpfung beim Kühlen, wobei bei etwa $200 \mathrm{~K}$ die Grenze der Genauigkeit der DPO Messung erreicht wird $\left(\approx 5 * 10^{-8}\right)$.

Die Messung der Dämpfung beim anschließenden Gegenheizen mit 1K/min ergibt ein mit der Abkühlmessung konsistentes Bild. Diese Messung ist in 


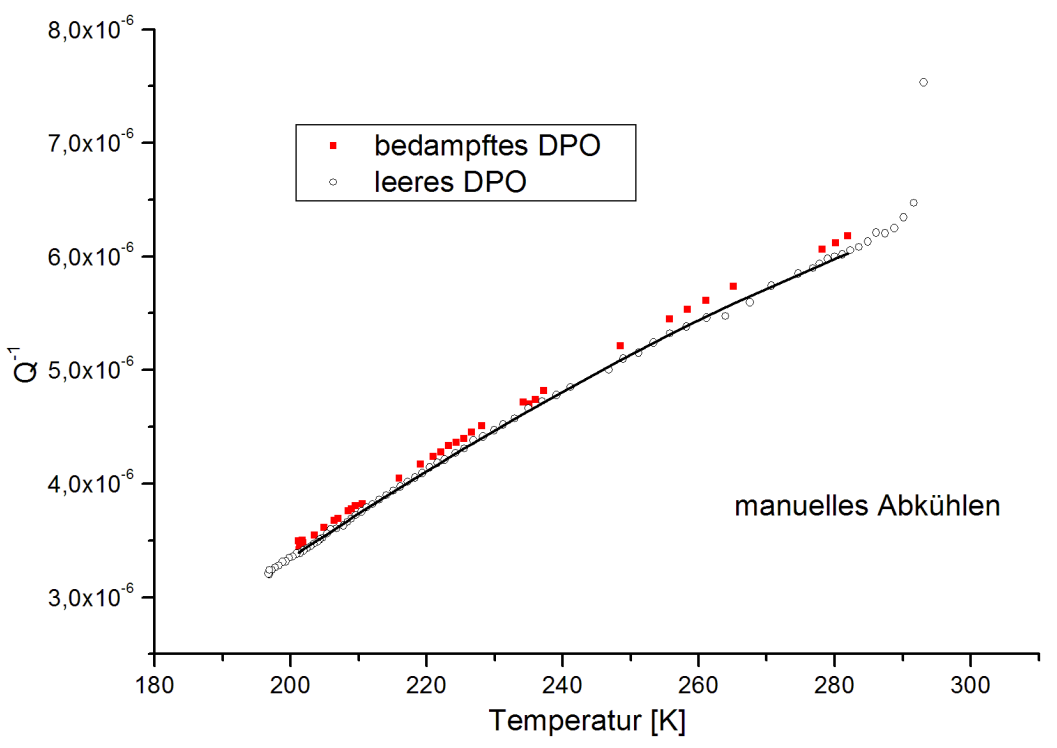

Abbildung 4.18: Messung der Dämpfung eines DPOs vergleichend mit $200 \mathrm{~nm}$ amorpher PdCuSi-Schicht und unbeschichtet. Für diese Messung wurde portionsweise mit fl. $\mathrm{N}_{2}$ gekühlt. Die schwarze Linie stellt eine Anpassung der Leermessung mit einem Polynom dar. Man erkennt in dieser Darstellung nur eine geringe Variation in der zusätzlichen Dämpfung.

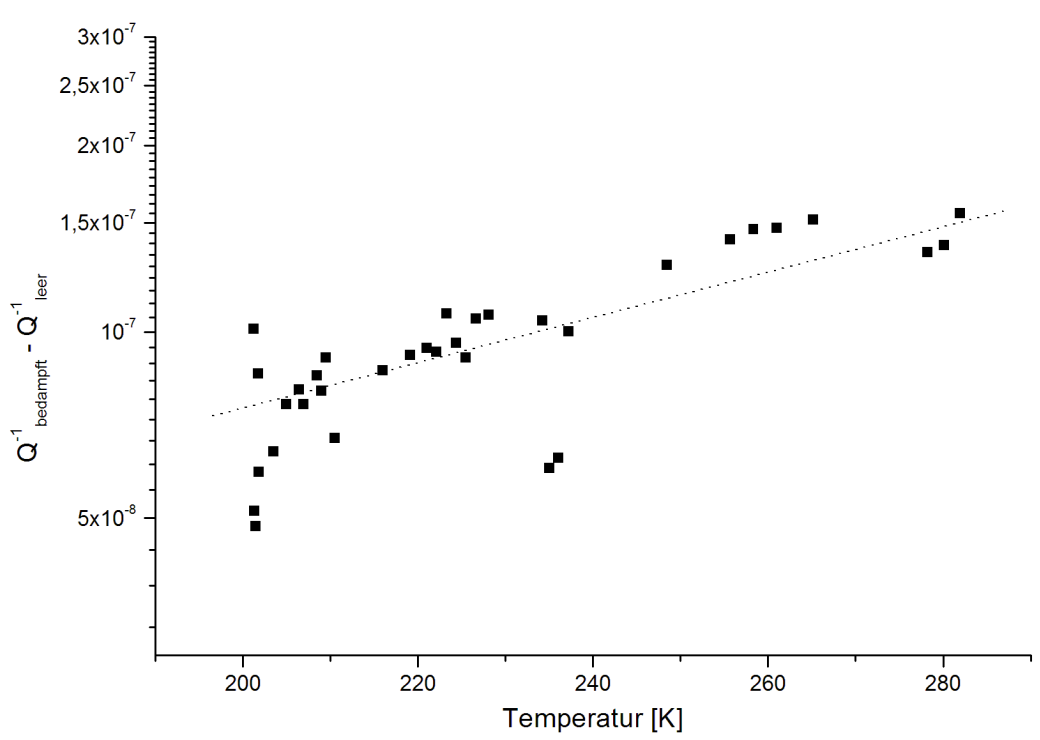

Abbildung 4.19: Dämpfungsdifferenz durch die $200 \mathrm{~nm}$ PdCuSi-Schicht beim Abkühlen unter Raumtemperatur. Die gestrichelte Linie stellt eine Trendlinie dar. 


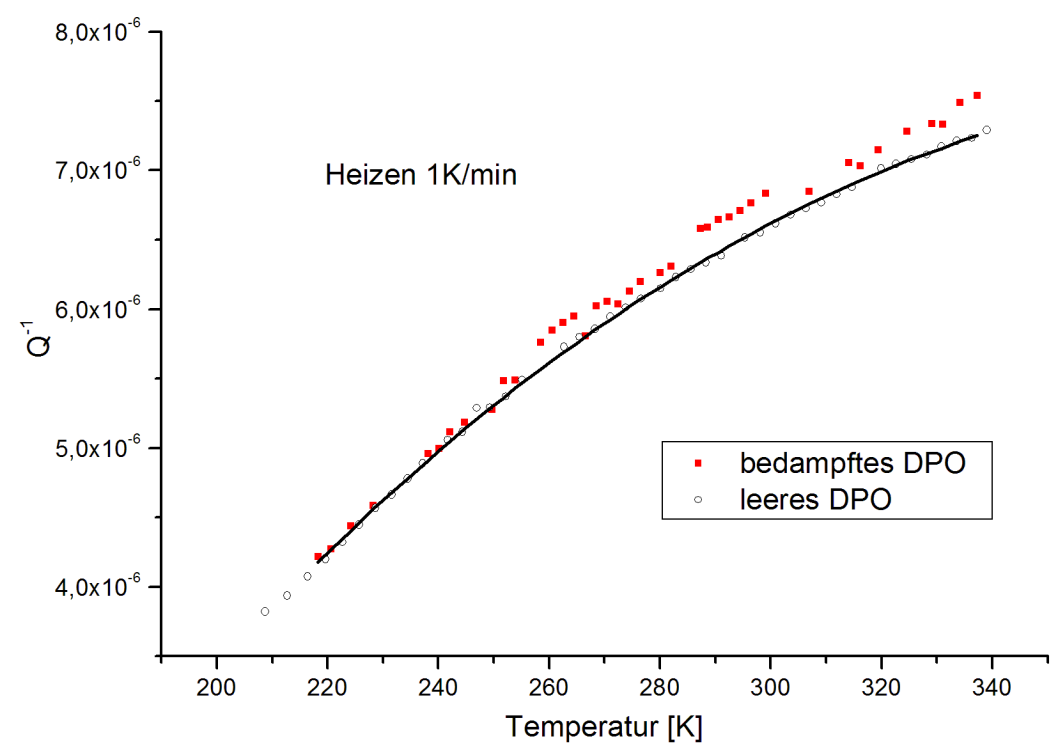

Abbildung 4.20: Messung der Dämpfung beim Heizen mit 1K/min beginnend bei $220 \mathrm{~K}$.

Abbildung 4.20 dargestellt. Bei der niedrigsten Temperatur liegt die Dämpfung nahe der Auflösungsgrenze dieser Methode und steigt mit zunehmender Temperatur deutlich an. Die genaue Bestimmung der Differenzdämpfung liefert vergleichbare Werte wie die Abkühlkurve, allerdings mit mehr Streuung.

Um zu testen, ob Relaxationen in diesem Temperaturbereich stattfinden, und auch um die Reproduzierbarkeit der Messungen zu prüfen wurde eine Kühlmessung wiederholt. Der Zustand der Schicht kann an der Frequenzverschiebung verfolgt werden. Die Messung der Resonanzfrequenz unter Raumtemperatur für das leere und auch bedampfte Paddel ist in Abbildung 4.21 gezeigt. Man erkennt, dass die Resonanzfrequenz durch den Film wie erwartet deutlich abgesenkt ist. Der Unterschied in der Resonanzfrequenz bleibt über den Temperaturbereich von $200 \mathrm{~K}$ bis $300 \mathrm{~K}$ fast konstant. Die genaue Vermessung der Frequenzabweichungen ergibt eine Reduzierung der Frequenzabsenkung von $5,2 \mathrm{~Hz}$ bei RT auf $4,8 \mathrm{~Hz}$ bei $200 \mathrm{~K}$. Die Anwendung von Gleichung (2.27) ergibt für den Schermodul der Schicht einen Anstieg von $33 \mathrm{GPa}$ auf $37 \mathrm{GPa}$ beim Kühlen.

Da sich beide Messdurchläufe im Ergebnis nicht signifikant unterscheiden, finden sich auch keine Hinweise auf irreversible Veränderungen im Film durch den ersten Messdurchlauf. 


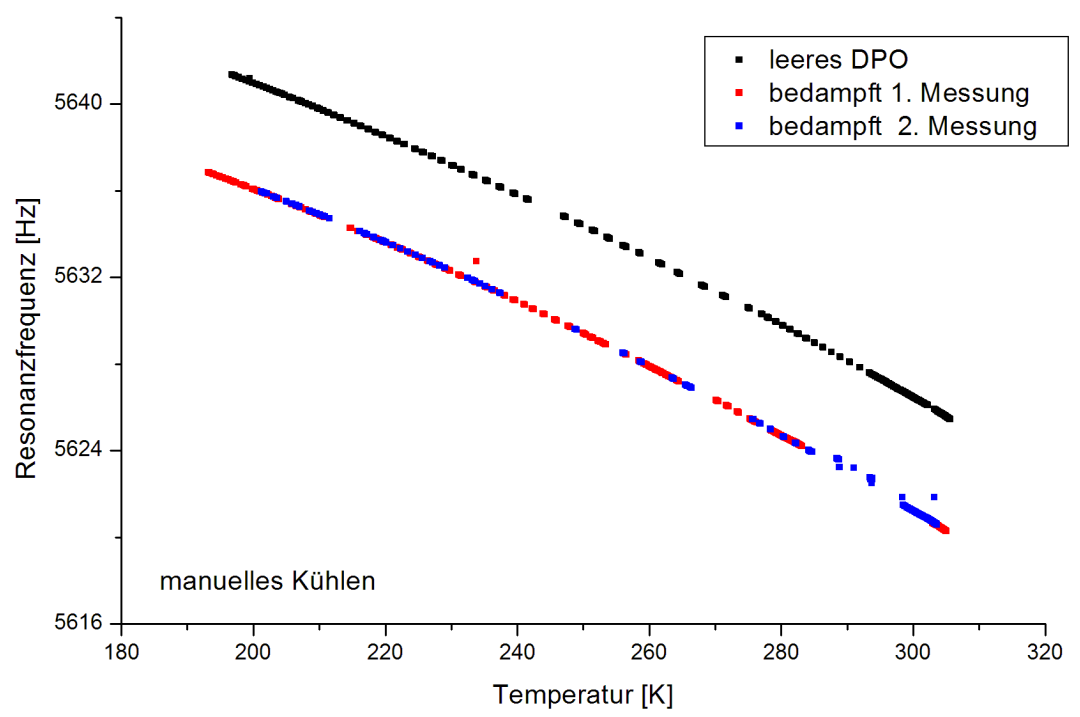

Abbildung 4.21: Messung der Frequenzlage des DPO_DB_05 mit einem amorphen PdCuSi-Film vergleichend mit dem leeren Paddel. Zwischen der ersten und der zweiten Messung wurde das Paddel mit Film mit 1K/min bis auf $340 \mathrm{~K}$ geheizt. Es finden sich keine signifikanten Unterschiede zwischen den zwei Messdurchläufen.

\subsubsection{Mechanische Charakterisierung dünner amorpher Filme}

\section{Auf rauen Substraten:}

Schichtdicke 50 nm: Die Ergebnisse der mechanisch vermessenen dünnen Schichten beginnen mit einer PdCuSi-Schicht, die auf einer rauen Unterlage deponiert wurde. Der Film DPO_DB_05a wurde auf den zuvor kristallisierten Film DPO_DB_05 aufgebracht.

Wie bereits in Kapitel 4.1.3 gezeigt, können kristallisierte und ausgelagerte Filme sehr rau ausfallen, die Topographie dieses amorphen Films ist daher nicht genau bekannt. Eine Diskussion dieses Aspektes erfolgt im Kapitel 5.5.1

Das Verlustspektrum dieses dünnen Films, das in Abbildung 4.22 gezeigt ist, unterscheidet sich bereits qualitativ deutlich von dem Verhalten des dicksten Films. Ein sehr breiter Verlauf, wie in Abbildung 4.13, fehlt in dieser Messung völlig. Bei etwa $590 \mathrm{~K}$ steigt das Dämpfungssignal mit Film über die Leermessung, um bereits bei $625 \mathrm{~K}$ auf diese zurückzufallen. Unter $590 \mathrm{~K}$ 


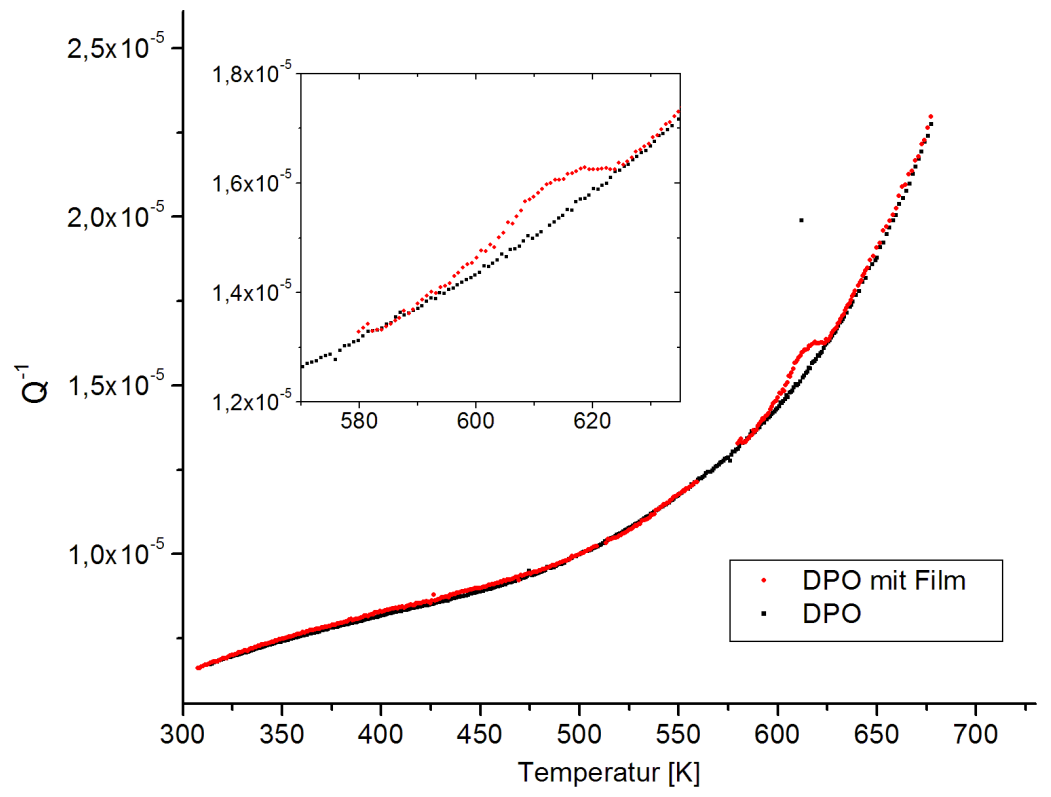

Abbildung 4.22: Messung des PdCuSi-Films von $50 \mathrm{~nm}$ auf rauem Substrat. Der Film wurde auf den zuvor kristallisierten Film DPO_DB_05 aufgebracht. Das Verlustspektrum zeigt über einen breiten Temperaturbereich eine kaum vom leeren Paddel abweichende Dämpfung. Erst bei etwa $590 \mathrm{~K}$ steigt die Dämpfung erkennbar an, um bereits bei $625 \mathrm{~K}$ wieder auf die Leermessung abzufallen. 
liegt die Dämpfung mit Film leicht oberhalb und auch unterhalb der Leermessung. Die Abweichungen sind nie größer als $1,5 * 10^{-7}$.

Es wurde der Versuch unternommen, weitere Filme auf dieses Paddel aufzubringen und $\mathrm{zu}$ vermessen, jedoch traten dabei Probleme in der Interpretierbarkeit der Messungen auf. Diese Messungen werden daher erst im Anhang A.2 gezeigt, eine Erörterung der Gründe für die Herausnahme an dieser Stelle findet sich in Kapitel 5.1.2. Um die Interpretation der Daten zu vereinfachen werden von nun an nur amorphe Filme auf glatten Substraten vorgestellt.

\section{Auf glatten Substraten:}

Schichtdicke 50 nm: Zunächst wird ein Film nominell gleicher Dicke wie zuvor auf ein glattes Substrat aufgebracht und vermessen. Das Verlustspektrum ist in Abbildung 4.23 gezeigt. Die Dämpfungsdifferenz zum leeren Paddel ist erheblich größer als für den Fall des rauen Substrates, zudem ist eine Differenzdämpfung über den gesamten gemessenen Temperaturbereich erkennbar.

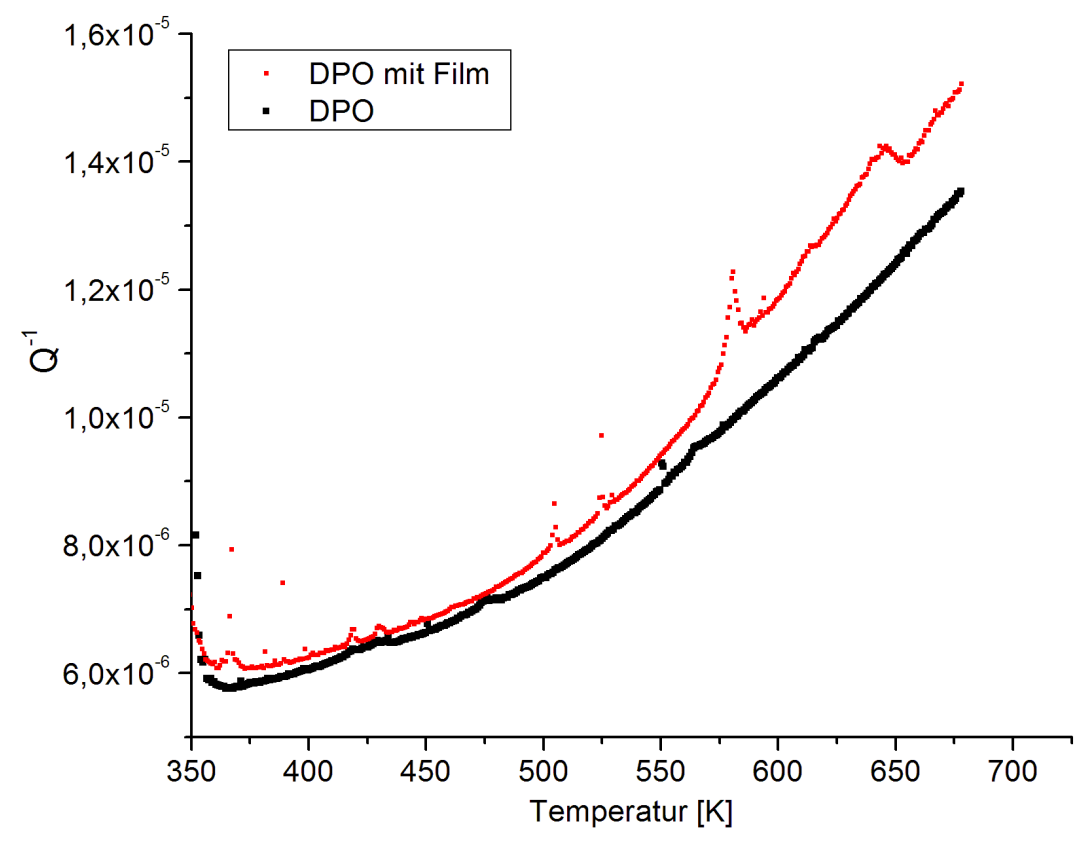

Abbildung 4.23: Dämpfungsverlauf des Paddels DPO_DB_07 mit $50 \mathrm{~nm}$ PdCuSi. Im Gegensatz zu DPO_DB_05a wurde dieser Film auf ein glattes Paddel aufgebracht.

Die scharfen Peaks, die bevorzugt in der Messung mit Film zu erkennen sind, traten auch in einigen Leermessungen desselben Paddels auf. In gleicher Wei- 
se wie für die dickste Schicht soll auch hier die subtrahierte Differenzdämpfung und die Frequenzverschiebung vorgestellt werden.

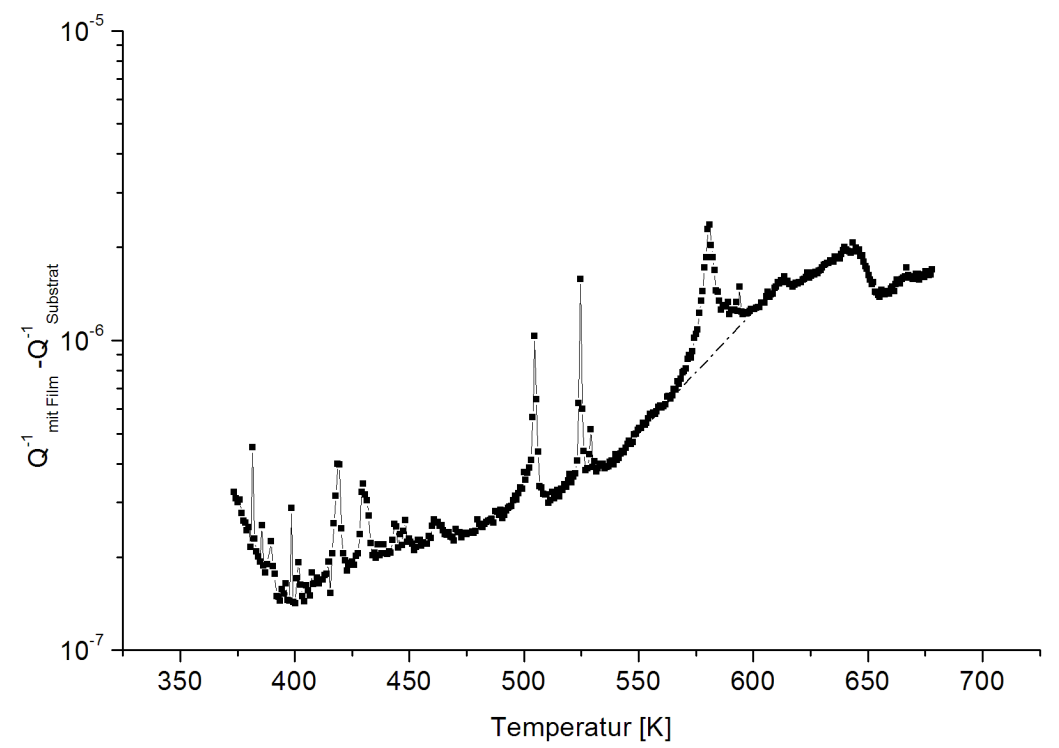

Abbildung 4.24: Dämpfung des $50 \mathrm{~nm} \mathrm{PdCuSi-Filmes} \mathrm{auf} \mathrm{dem} \mathrm{glatten} \mathrm{Sub-}$ strat. Die Dämpfung nimmt beim Heizen zunächst stark ab und steigt bereits ab $400 \mathrm{~K}$ kontinuierlich an. Bei $645 \mathrm{~K}$ reduziert sich die Dämpfung schnell um anschließend wieder leicht anzusteigen. Die scharfen Peaks treten auch auf den Leermesungen des Paddels auf. Die gestrichelte Linie bei $\approx 600 \mathrm{~K}$ stellt einen gedachten Verlauf ohne den scharfen Peak in diesem Temperaturbereich dar.

Der Verlauf der Differenzdämpfung (Abb. 4.24) weist, genau wie die eigentliche Messung mit Film, eine Vielzahl von scharfen Dämpfungspeaks auf, die jedoch auch für das leere Paddel zu beobachten sind. Bei $\approx 600 \mathrm{~K}$ verdeutlicht die gestrichelte Linie einen möglichen Verlauf ohne den starken Peak. Vergleicht man diese Messung mit Abbildung 4.14, so erkennt man eine große Ähnlichkeit bezüglich des Verlaufs. Das Absinken und auch Ansteigen der Dämpfung mit der Temperatur verläuft in gleicher Weise wie für den dicksten gemessenen Film.

Die Frequenzverschiebung, die den Temperaturverlauf des Schermoduls der Schicht widerspiegelt, ist in Abbildung 4.25 dargestellt. Das Bild ist ebenfalls sehr ähnlich zu der Messung der dicksten Schicht (vgl. Abb. 4.16). Auch hier verringert sich zunächst die Frequenzverschiebung, erreicht ein Minimum und steigt über einen großen Temperaturbereich wieder an. Ab $625 \mathrm{~K}$ erhöht sich die Frequenzverschiebung stark. Die Auftragung wurde so gewählt, dass ein 
Ansteigen des Graphen einem Anstieg des Schermoduls der Schicht nach Gleichung (2.27) entspricht.

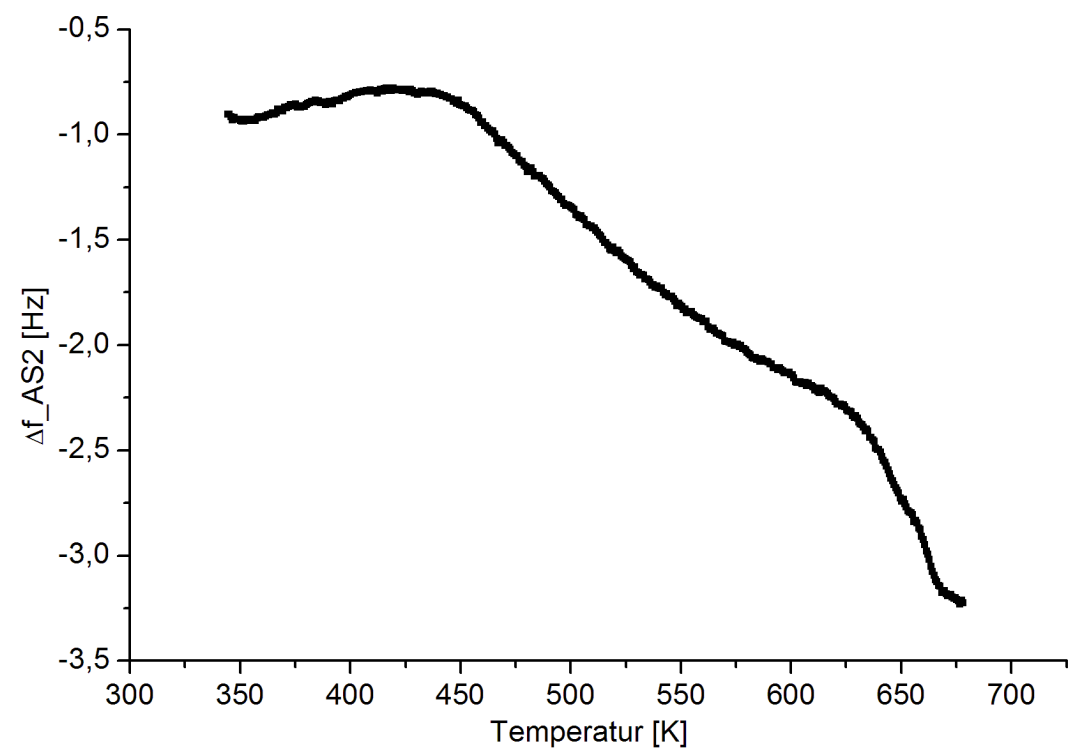

Abbildung 4.25: Frequenzverschiebung durch den 50 nm PdCuSi-Film. Man erkennt einen Anfangsbereich mit einem Rückgang der Frequenzverschiebung und dann einen langsamen Anstieg der Frequenzverschiebung. Ab $625 \mathrm{~K}$ verläuft dieser deutlich steiler.

Schichtdicke 25 nm: Auf ein neues Paddel wurde ein PdCuSi-Film mit nominell $25 \mathrm{~nm}$ Dicke (DPO_DB_07) aufgedampft. Die Dämpfung des Paddel mit dem Film beim ersten Aufheizen im Vergleich zum leeren Paddel und auch vergleichend mit dem kristallisierten Film zeigt Abbildung 4.26.

In dieser Auftragung erkennt man keine Dämpfung durch den Film unterhalb von $550 \mathrm{~K}$, anschließend steigt sie kontinuierlich an. Die Dämpfung geht erst bei sehr hoher Temperatur auf die Leermessung zurück. Die in blau dargestellte Messung des Paddels mit kristallisierter Schicht zeigt über den gesamten Temperaturbereich keine zusätzlich messbare Dämpfung. Auch bei diesem Messdurchlauf konnten scharfe Dämpfungspeaks nicht vermieden werden, sie treten sowohl in der Leermessung als auch in den Messungen der amorphen und kristallinen Schicht auf. Für diesen Messdurchlauf wirken sich die Dämpfungsereignisse so stark aus, dass auch die Frequenzmessungen davon beeinflusst werden. Die Frequenzverschiebung durch diese Schicht kann daher nicht als Funktion der Temperatur bestimmt werden. 


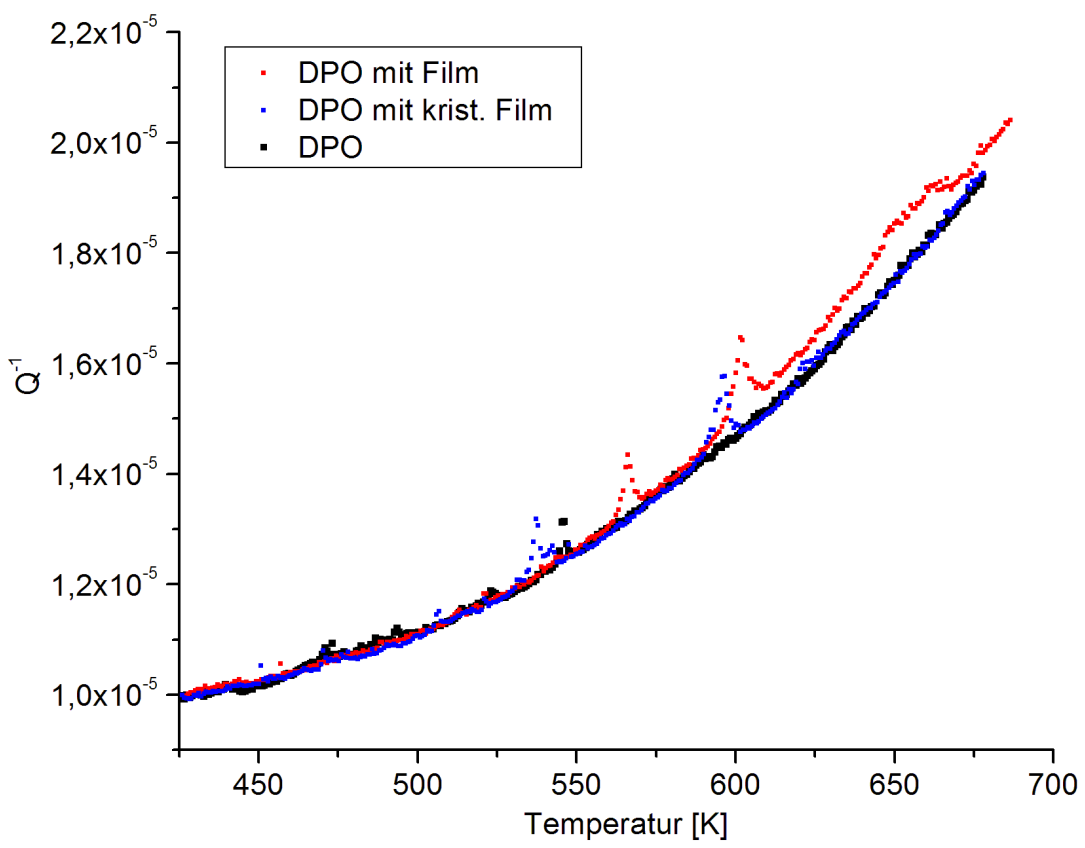

Abbildung 4.26: Messung der Dämpfung mit $25 \mathrm{~nm}$ PdCuSi. Dargestellt ist sowohl das erste Aufheizen des amorph hergestellten Films (rot) als auch eine Messung nach der Kristallisation des Films (blau). Unterhalb von $550 \mathrm{~K}$ ist keine Dämpfungsdifferenz zum leeren Paddel auszumachen. 
Vergleich: An dieser Stelle sollen die bisherigen Messergebnisse zu den Schichten auf den glatten Substraten untereinander verglichen werden. Dazu stellt Abbildung 4.27 die Dämpfung der Schichten in einem Graphen gemeinsam dar.

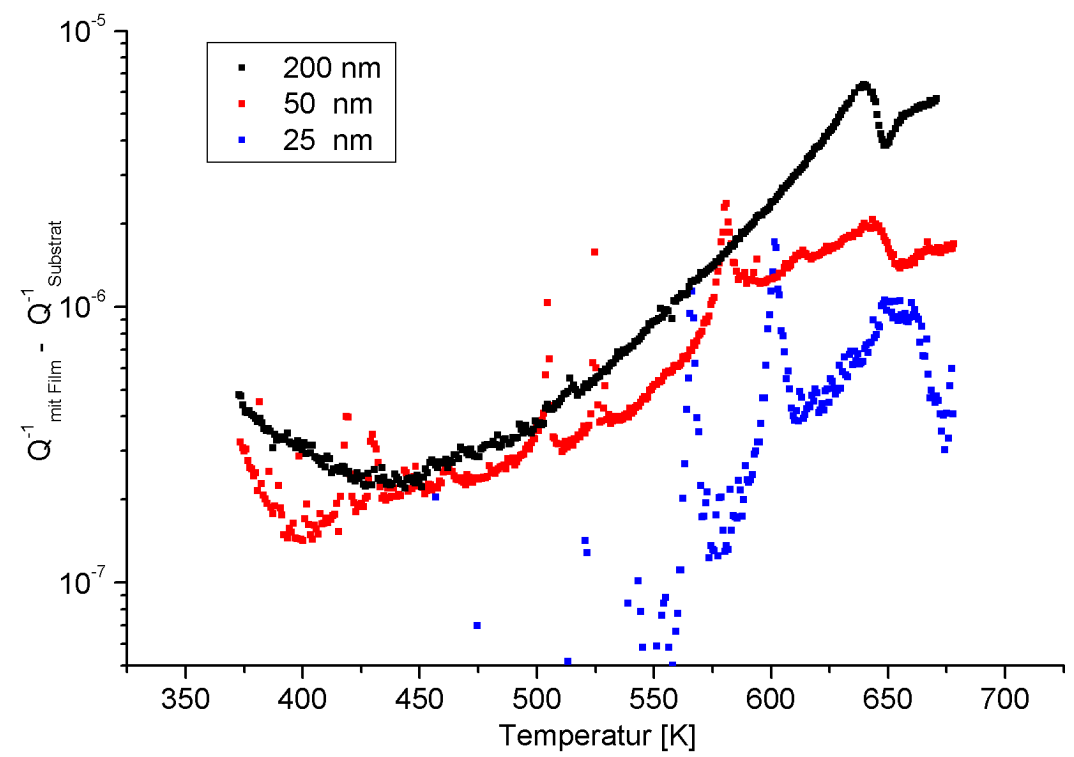

Abbildung 4.27: Übersicht der Verlustmessungen an Schichten verschiedener Dicken auf glatten Substraten. Die y-Achse beginnt bei der Grenze der Messgenauigkeit.

Man erkennt in dieser Übersicht zunächst vier qualitative Unterschiede:

1. Der Temperaturbereich unter $550 \mathrm{~K}$ der dünnsten Schicht weist überhaupt keine messbare Dämpfung auf. Skaliert man den gemessenen Wert der Schicht mit $50 \mathrm{~nm}$ Dicke um einen Faktor zwei herunter, so liegt man noch deutlich im messbaren Bereich des Verlustes.

2. Der Anstieg im Verlust zu höheren Temperaturen ist für die dünnste Schicht deutlich steiler als bei den dickeren Schichten. Der Verlauf des Verlustes in den beiden dickeren Schichten scheint sich zu tiefen Temperaturen hin etwas abzuflachen. Ein solches Verhalten wird bei der dünnsten Schicht ebenfalls nicht beobachtet.

3. Die gemessene Dämpfung bei hoher Temperatur skaliert in etwa mit der Schichtdicke. 
4. Der Einbruch in der Dämpfung am Ende der Messung verschiebt sich bei dünner werdenden Schichten kontinuierlich zu höherer Temperatur. Dieser Zusammenhang wird in Kapitel 5.5.4 quantifiziert.

Schichtdicke 10 nm: Als dünnste Probe wurde eine Schichtdicke von nur $10 \mathrm{~nm}$ untersucht. Bereits bei den vorhergehenden Analysen hat diese Schicht stets eine Sonderolle eingenommen, auch die mechanische Analyse war in diesem Fall besonders herausfordernd. Die Ergebnisse kontrastieren mit den bisherigen und werden in Kapitel 5.5.3 gesondert diskutiert.

Abbildung 4.28 zeigt für diese Probe drei Messdurchläufe: In rot dargestellt ist der Dämpfungsverlauf mit der frisch hergestellten Schicht, in blau die kristallisierte Schicht und schwarz das leere Doppelpaddel. Auffällig ist ein ausgeprägter Verlust mit der frischen Schicht gegenüber den beiden anderen Messdurchläufen. Dieser Unterschied erstreckt sich auch über ein breites Temperaturintervall. Im unteren Temperaturbereich ist diese Abgrenzung schwierig, da unterhalb von $500 \mathrm{~K}$ dieses Paddel irreguläre Dämpfung aufweist. Man erkennt kein direktes Einknicken der Dämpfung mit Film, jedoch nähert sich die Dämpfung mit Film dem Verlauf ohne Film wieder an. Bei etwa $690 \mathrm{~K}$ unterscheiden sich der erste und der zweite Messdurchlauf mit Film nicht mehr voneinander.

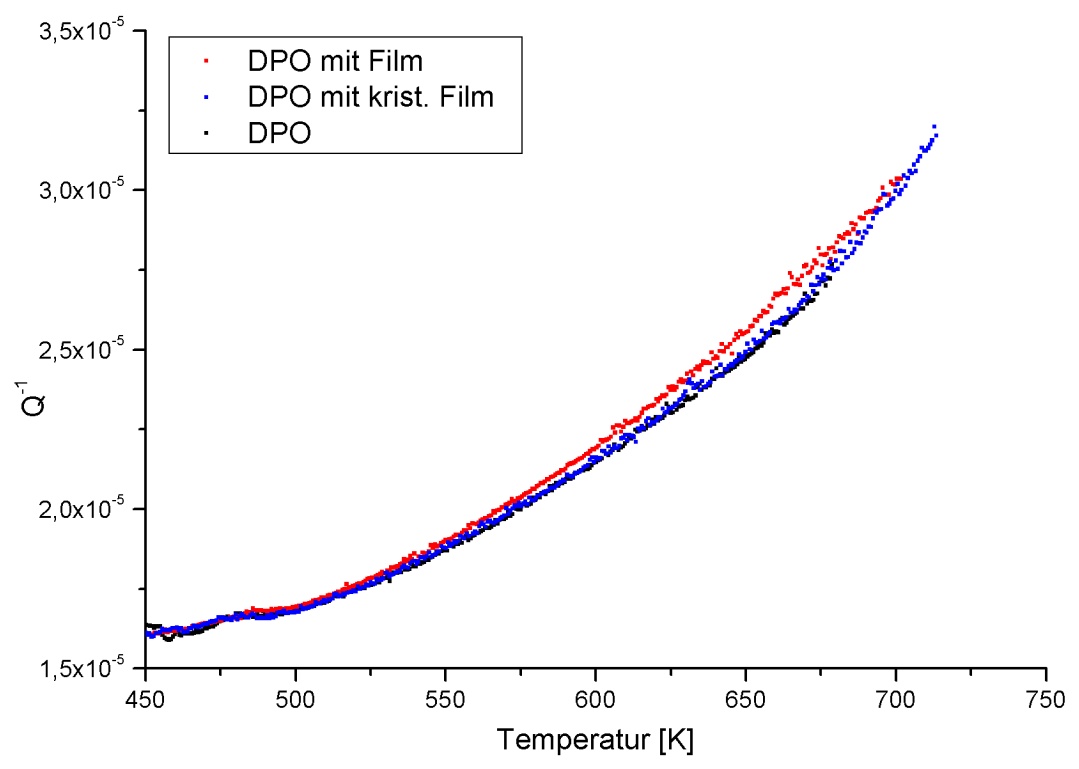

Abbildung 4.28: Messung der Dämpfung mit 10 nm PdCuSi (rot), mit kristallisiertem Film (blau) und ohne Film (schwarz).

Der Verlust in der Schicht wurde aus der Abweichung zu Leermessung und zweitem Messdurchlauf (DPO mit krist. Film) bestimmt. Das Ergebnis zeigt 
Abbildung 4.29. Es zeigen sich hohe absolute Werte in der Dämpfung. Der Verlauf unterhalb von $500 \mathrm{~K}$ kann nicht sicher angegeben werden.

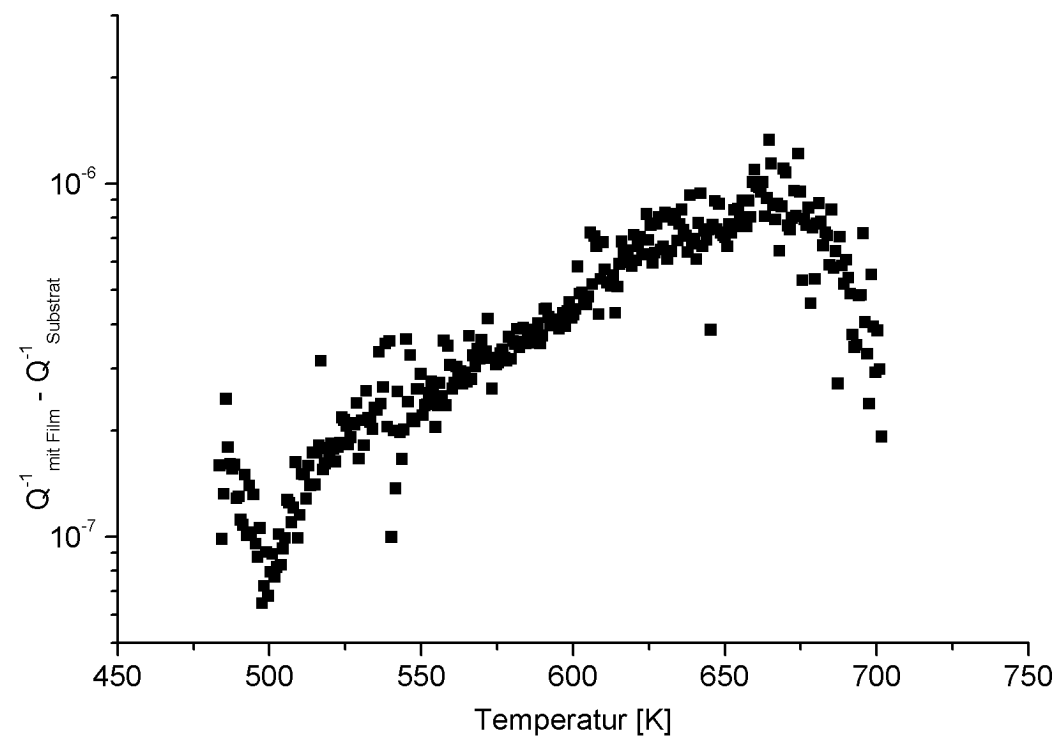

Abbildung 4.29: Verlust durch die $10 \mathrm{~nm}$ PdCuSi-Schicht. Für $Q_{\text {Substrat }}^{-1}$ wurde die Leermessung und die Messung mit krist. Film verwendet.

Die Frequenzverschiebung konnte für diesen Film bestimmt werden, der Verlauf ist in Abbildung 4.30 gezeigt. Die Frequenzverschiebung reduziert sich anfänglich, erreicht ein Minimum und steigt anschließend kontinuierlich. Ein Vergleich zu den bisherigen Messungen, gezeigt in Abbildung 4.16 und 4.25, zeigt das Fehlen des raschen Anstiegs in $\Delta f_{A S 2}$, der nach Gleichung (2.27) einer Reduzierung des Schermoduls entspricht. Zu beachten sind die relativ kleinen Werte der Frequenzverschiebung. Die Gleichung (2.27) ergibt für diese Schichtdicke und Dichte ein Limit von $-0,4 \mathrm{~Hz}$ für einen Schermodul von Null.

Um den Verlauf des Schermoduls dieser Schicht genauer zu untersuchen, wird ein Vergleich mit dem kristallisiertem Film herangezogen. Abbildung 4.31 zeigt den Unterschied in der Resonanzfrequenz zwischen dem ersten Messdurchlauf des Films und dem zweiten Messdurchlauf. ${ }^{7}$

Aus diesem Vergleich erkennt man, dass die Frequenz mit frischem Film unterhalb der Frequenz mit kristallinem Film beginnt und sich bis $550 \mathrm{~K}$ noch etwas absenkt. Ab $550 \mathrm{~K}$ reduziert sich der Unterschied beim Heizen. Bei 665 K steigt die Frequenz steil über die Resonanzfrequenz mit Film und

\footnotetext{
${ }^{7}$ Damit entfällt der Anteil der Massenbelegung an der Frequenzverschiebung.
} 


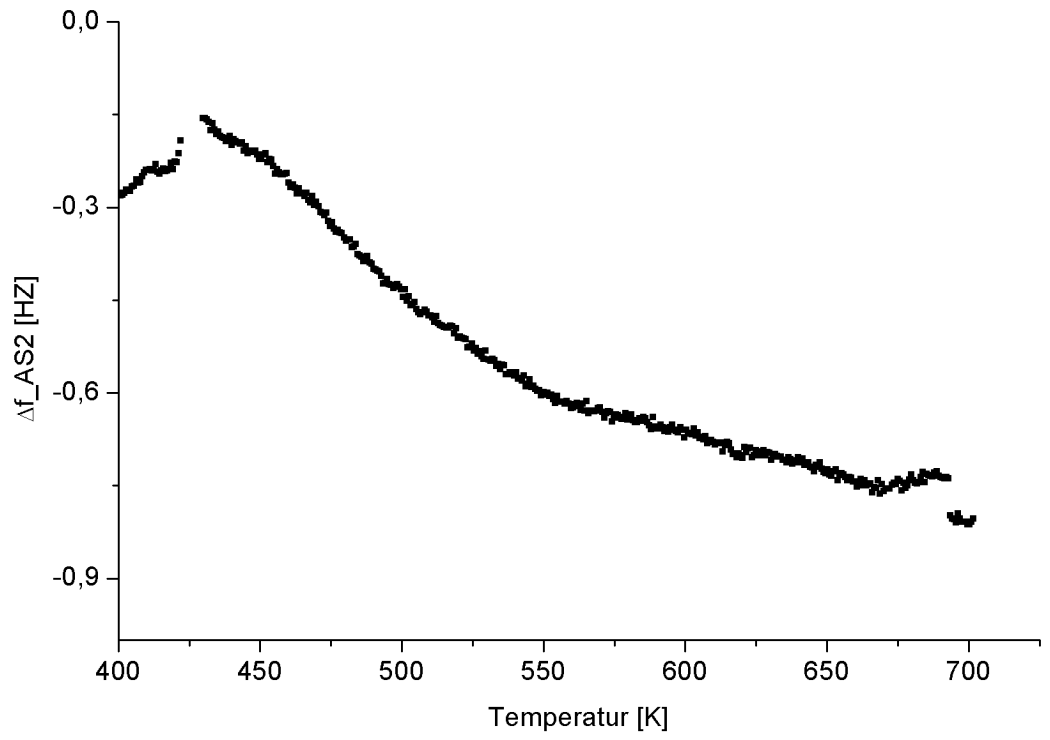

Abbildung 4.30: Frequenzverschiebung durch den 10 nm PdCuSi-Film.

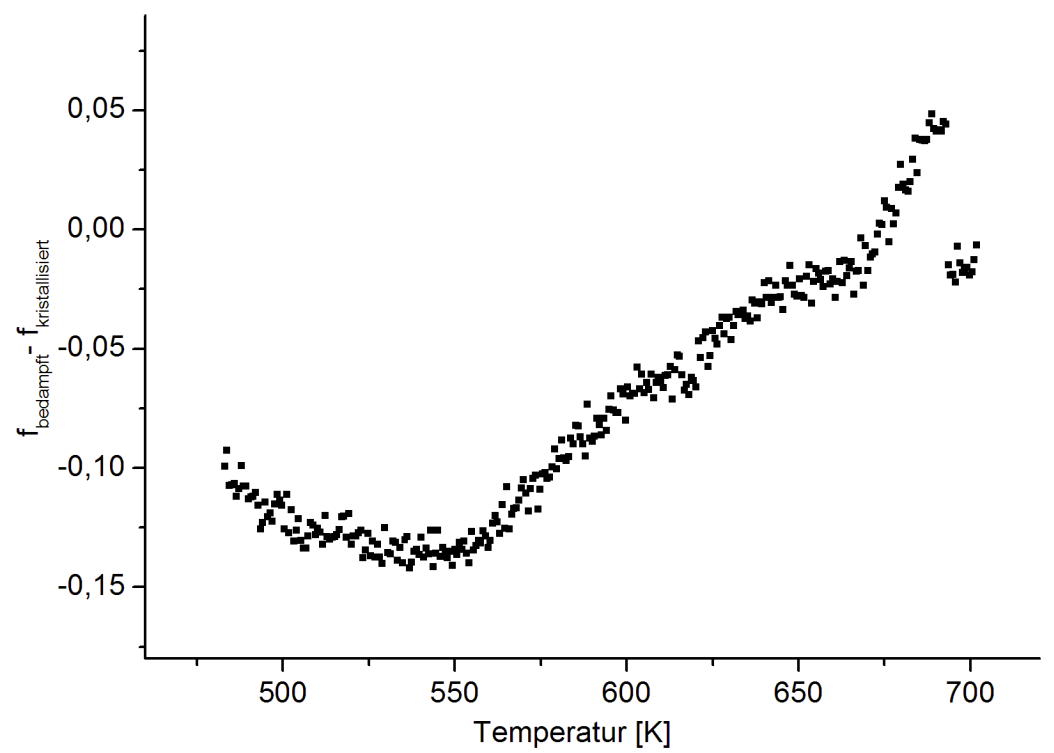

Abbildung 4.31: Frequenzunterschied zwischen dem ersten Messdurchlauf der $10 \mathrm{~nm}$ Schicht und dem zweiten mit kristallisiertem Film. 
fällt bei ca. $695 \mathrm{~K}$ abrupt auf einen Wert nahe Null. Die Abweichungen der Messwerte von dem zur Subtraktion verwendeten Fit liegen bei unter 0,02 Hz.

\subsubsection{Einfluss der thermischen Vorbehandlung}

Alle bisher gezeigten Messungen sind an den frisch hergestellten Schichten vorgenommen worden. Diese spektroskopischen Messungen des ersten Aufheizens der Proben bis über die Kristallisation können prinzipiell nicht von den gleichzeitig ablaufenden Prozessen durch die Temperaturbehandlung separiert werden. Der Einfluss einer thermischen Vorbehandlung wird in Abbildung 4.32 am Beispiel einer Schicht von 100 nm Dicke gezeigt, für die der erste Messdurchlauf deutlich vor der Kristallisation gestoppt wurde. Nach dem Abkühlen auf Raumtemperatur folgte ein zweiter Messdurchlauf zu deutlich höherer Temperatur. Das hierbei verwendetete Paddel DPO_DB_04 weist keine Tantalschicht auf, zudem wurde eine doppelt so hohe Heizrate $(2 \mathrm{~K} / \mathrm{min})$ gewählt. Das Spektrum ist damit nur bedingt mit den anderen Messungen in dieser Arbeit vergleichbar. Wie in Abbildung $4.32 \mathrm{zu}$ erkennen, erfolgte die erste Messung nur bis etwa $625 \mathrm{~K}$. In diesem Temperaturbereich weist das Paddel mit Schicht eine deutliche Dämpfung gegenüber dem leeren Paddel auf. Im zweiten Durchlauf ist im gleichen Temperaturbereich keine Dämpfung mehr nachweisbar, bei etwa $600 \mathrm{~K}$ scheint die Dämpfung anzusteigen, es fehlt aber der Vergleich mit einer Leermessung. Der weitere Verlauf erscheint vergleichbar zu dem Spektrum der dicksten Schicht (vgl. Abb. 4.13), lediglich der Einbruch in der Dämpfung ist ausgeprägter und liegt bei einer höheren Temperatur.

\subsubsection{Mechanische Eigenschaften nahe Raumtempera- tur}

Vor und nach jedem Beschichtungsvorgang, sowie nach der Kristallisationsmessung wurden Frequenzverschiebung und Dämpfungsänderung bei konstanten $40{ }^{\circ} \mathrm{C}$ Blocktemperatur gemessen. Diese Temperatur entspricht einer Paddelkopftemperatur von etwa $316 \mathrm{~K}$ und kann sehr genau eingeregelt werden. Das hierzu verwendete Verfahren „Anfahren und Warten“ ist in Kapitel 3.1.1 beschrieben. Nach dem thermischen Einschwingen des Messaufbaus, exemplarisch gezeigt in Abbildung 3.4, werden fortlaufend Messewerte für Frequenz und Güte des Paddels aufgenommen. Dies erlaubt eine, im Vergleich zu Heizrampen, genauere Bestimmung dieser Eigenschaften durch Mittelung der Messungen. 


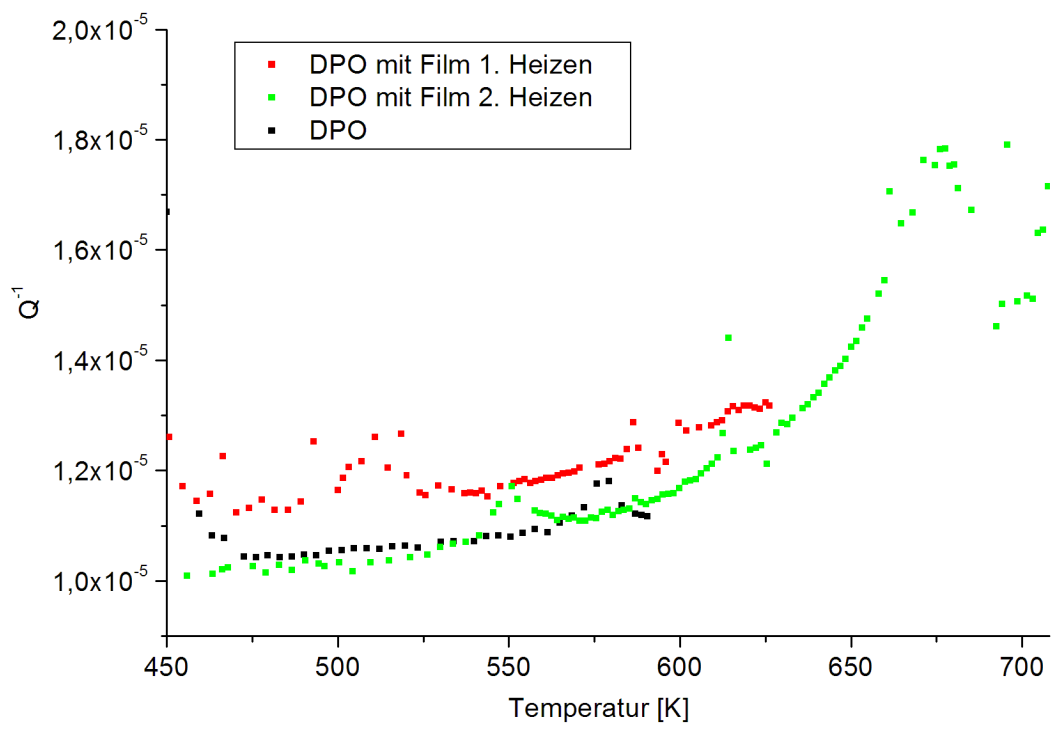

Abbildung 4.32: Einfluss der therm. Vorbehandlung auf das Spektrum. Der erste Messdurchlauf wurde bei $625 \mathrm{~K}$ gestoppt. Nach dem Abkühlen auf Raumtemperatur erfolgte der zweite Messdurchlauf.

\section{Schermodul:}

Der Schermodul der frisch hergestellten Schichten wird nach Gleichung (2.27) aus der Frequenzverschiebung des Paddels, der gemessenen Schichtdicke auf dem Referenzsubstrat und der Schichtdichte berechnet. Die Dichteberechnung erfolgt nach Gleichung (2.28) aus den Ergebnissen der EDX-Messungen. Der Schermodul wird also aus mindestens drei unabhängigen Messungen bestimmt, die Fehler können entsprechend groß ausfallen. Führt diese Berechnung aber zu einem realistischen Wert für $G_{F i l m}$, so ergeben alle Messungen ein konsistentes Bild der hergestellten Schicht.

Die Tabelle 4.3 zeigt diese Ergebnisse in einer Übersicht.

Diese Übersicht zeigt für die hergestellten Schichten einen Schermodul von etwa $35 \mathrm{GPa}$, der bei einem abgeschätzten Fehler von 2-3 GPa für fast allen Schichten zutrifft. Die Probe DPO_DB_04 weicht hiervon ab, jedoch weist dieses Doppelpaddel keine Tantalbarriere auf, und die Frequenzmessung mit Schicht wurde erst nach einem Kühldurchlauf durchgeführt. Die dünnste Schicht weicht von dem mittleren Wert sehr deutlich ab, der mögliche Fehler dieses Wertes ist aber auch erheblich größer. Berechnet man beispielsweise das Schermodul unter der Annahme einer Schichtdicke von $12 \mathrm{~nm}$ anstelle von 10,5 nm, so ergibt sich allein durch diese kleine Variation ein Schermodul von $30 \mathrm{GPa}$. Auch bei den anderen Modulberechnungen 


\begin{tabular}{|c|c|c|c|}
\hline Probe & $\begin{array}{c}\text { Schichtdicke } \\
{[\mathrm{nm}] \text { (XRD) }}\end{array}$ & $\begin{array}{c}G_{\text {Film }} \text { (aufgedampft) } \\
{[\mathrm{GPa}]}\end{array}$ & $\begin{array}{c}G_{\text {Film }} \text { (krist.) } \\
{[\mathrm{GPa}]}\end{array}$ \\
\hline DPO_DB_05 & 200 (nom.) & 32 & 55 \\
\hline DPO_DB_04 $(\mathrm{x})$ & 93 & 19 & 80 \\
\hline DPO_DB_07 & 55 & 38 & 65 \\
\hline DPO_DB_05a & 52 & 37 & 64 \\
\hline DPO_DB_08 & 27,5 & 35 & 71 \\
\hline DPO_DB_13 & 10,5 & 21 & 53 \\
\hline
\end{tabular}

Tabelle 4.3: Berechnete Schermodule der hergestellten Schichten nach dem Aufdampfen und nach der Kristallisationsheizmesssung. Die Fehler der Angaben liegen in der Größenordnung von 2-3 GPa, mit Ausnahme der dünnsten Schicht. Die Angabe der größten Schichtdicke entspricht einen nomineller Wert. Die Markierung (x) kennzeichnet ein Paddel ohne Tantalschicht.

stellt die Unsicherheit der Schichtdicke die größte Fehlerquelle dar.

Die Schermodule der Schichten nach der Kristallisationsmessung liegen um $65 \mathrm{GPa}$ und variieren etwas stärker als die frisch hergestellten Schichten. Sehr deutlich ist der Unterschied im Modul von aufgedampft zu nach der Kristallisationsheizmessung.

\section{Schichtdämpfung:}

Ein zusätzlicher mechanischer Verlust durch die dünnen Schichten kann bereits bei Raumtemperatur, bzw. bei $316 \mathrm{~K}$, festgestellt werden. Diesen zu vermessen stellt die höchsten Anforderungen an die mechanische Stabilität des Messaufbaus, da kontinuierlich bei fester Temperatur über Stunden gemessen wird. Nicht bei allen Doppelpaddeln ist dies möglich gewesen, es konnten dennoch ausreichend Messungen gewonnen werden, um Änderungen mit der Schichtdicke zu zeigen. Bei einer typischen Standardabweichung von $5^{*} 10^{-8}$ der $Q^{-1}$-Messwerte um einen Mittelwert, kann dieser aus 20 Messwerten auf bis zu $1^{*} 10^{-8}$ genau bestimmt werden. Für die Bestimmung von $\Delta Q^{-1}$ ergibt sich damit ein Fehler von etwa $2^{*} 10^{-8}$. Bei einigen Doppelpaddeln zeigen sich starke Dämpfungspeaks nahe oder bei $40^{\circ} \mathrm{C}$ Blocktemperatur bzw. Sprünge in der Dämpfung über der Messzeit. In diesen Fällen wird in Tabelle 4.4 ein „n. b.“ für nicht bestimmbar angegeben. Die Tabelle 4.4 zeigt die bestimmten Dämpfungen der Schichten, unabhängig ob die Schicht auf rauen (DPO_DB_05a, DPO_DB_05b und DPO_DB_05c) oder glatten Substraten deponiert wurden. 


\begin{tabular}{|c|c|c|c|}
\hline Probe & $\begin{array}{c}\text { Schichtdicke } \\
{[\mathrm{nm}]}\end{array}$ & $Q_{\text {bed. }}^{-1}-Q_{\text {Sub. }}^{-1}$ & $Q_{\text {bed. }}^{-1}-Q_{\text {krist. }}^{-1}$ \\
\hline DPO_DB_05 & 200 (nom.) & $6,46^{*} 10^{-7}$ & $7,61^{*} 10^{-7}$ \\
\hline DPO_DB_05b & 110 & $9,51^{*} 10^{-8}$ & $3,36^{*} 10^{-8}$ \\
\hline DPO_DB_04(x) & 93 & $9,52^{*} 10^{-7}$ & $2,38^{*} 10^{-6}$ \\
\hline DPO_DB_07 & 55 & n. b. & n. b. \\
\hline DPO_DB_05a & 52 & $8,82^{*} 10^{-8}$ & $6,55^{*} 10^{-8}$ \\
\hline DPO_DB_08 & 27,5 & n. b. & n. b. \\
\hline DPO_DB_05c & 22 & $1,51^{*} 10^{-8}$ & $1,66^{*} 10^{-8}$ \\
\hline DPO_DB_13 & 10,5 & n. b. & n. b. \\
\hline
\end{tabular}

Tabelle 4.4: Dämpfung durch die hergestellten Schichten nahe Raumtemperatur $(316 \mathrm{~K})$. Dargestellt ist der Unterschied mit bedampften Schichten (bed.) zum leeren Paddel (Sub.) und auch der Dämpfungsunterschied zur Messung nach der Kristallisationsheizmessung (krist.). Die Angabe der größten Schichtdicke entspricht einen nomineller Wert. Die Markierung (x) kennzeichnet ein Paddel ohne Tantalschicht.

Bei einem Vergleich der Dämpfungsänderungen fällt die Differenz an der Probe DPO_DB_04 als überproportional stark auf, jedoch gelten auch hier die gleichen Einschränkungen in der Vergleichbarkeit wie bei den zuvor gezeigten Schermodulbestimmungen. Mit dieser einen Ausnahme erkennt man eine Abnahme der Dämpfung mit abnehmender Schichtdicke, jedoch keine lineare Abhängigkeit.

Die Differenzen zu den Messungen nach den Kristallisationsheizmessungen sind vergleichbar mit dem Dämpfungsanstieg der frischen Schicht zum leeren Paddel $\left(Q_{\text {bedampft }}^{-1}-Q_{\text {Substrat }}^{-1}\right) \approx\left(Q_{\text {bedampft }}^{-1}-Q_{\text {krist. }}^{-1}\right)$. 


\section{Kapitel 5}

\section{Diskussion}

Die Diskussion der Ergebnisse gliedert sich wie folgt:

Zuerst werden die hergestellten dünnen Schichten, in Bezug auf Zusammensetzung, Reproduzierbarkeit und die Beeinflussung durch Rauigkeiten der Grenzflächen diskutiert. Dem folgt eine Erörterung der methodischen Voraussetzungen der Messmethode und eventuelle Einschränkungen.

Anschließend können die Ergebnisse der dicksten Schicht bewertet werden. Es wird aufgezeigt, welche neuen Erkenntnisse mit dem fragilen Glas in Form einer aufgedampften Schicht vergleichend zu früheren Arbeiten gewonnen werden können.

In einer Zusammenfassung der Ergebnisse der Kühlmessungen, der Raumtemperaturergebnisse der Schichten und einiger weiterer Messungen an metallischen Gläsern bei tiefen Temperaturen wird ein konsistentes Bild der Verlustprozesse im Temperaturbereich unter der Glastemperatur erstellt.

Der Schwerpunkt liegt dann auf der Analyse der Messungen hin zu den dünnsten Schichten. Die Ergebnisse werden im Abschnitt 5.5 vergleichend diskutiert und anschließend eine Modellvorstellung zur Erklärung vorgeschlagen. Es folgt eine Einordnung dieser Ergebnisse in den aktuellen Stand der Erforschung von Längenskaleneffekten in Gläsern und kristallinen Materialien.

\subsection{Qualität der Schichten, Morphologie}

\subsubsection{Stöchiometrie}

Die Schichtzusammensetzung wird aus den Referenzsubstraten bestimmt. Der Abstand des Referenzsubstrates zum DPO bei der Deposition beträgt $<1 \mathrm{~cm}$, während der Abstand zu den Elektronenstrahlverdampfern etwa 
$30 \mathrm{~cm}$ beträgt. Damit können die Zusammensetzungsergebnisse ohne weitere Einschränkungen auf die Schichten auf den Paddeln übertragen werden.

Die Übersicht der Schichtzusammensetzungen (Tabelle 4.1) zeigt für alle Schichten einen kleineren Palladium-Anteil (73-74 at.\%) als in der Soll-Zusammensetzung $\mathrm{Pd}_{77,5} \mathrm{Cu}_{6,0} \mathrm{Si}_{16,5}$. Der Grund dafür ist nicht erkennbar, da der aktuelle Toolingfaktor, der die Legierung trotz kontrollierter Rate auf den Schwingquarzwaagen verfälschen kann, mehrfach kontrolliert wurde und stets konsistente Werte aufwies. Es ist möglich, dass die Messung mittels EDX ein nicht ganz korrektes Ergebnis liefert, da die Parameter der Auswertesoftware „Noran System SIX“ für massives Material und nicht für dünne Filme bestimmt sind. Wie in Abbildung 4.2 gezeigt, stammen die meisten Röntgenquanten von den Substraten der Filme. Auch mit den leicht abweichenden Zusammensetzungen, sind für die bisher vorgestellten Schichten keine gravierenden Einschränkungen in der Stabilität gegen Kristallisation zu erwarten. In einer Studie von L. F. Chua et al. wurde die Glasbildung von PdCuSi für verschiedene Palladium- zu Kupfer-Verhältnisse untersucht [70]. Dabei wird bis zu einer Palladiumuntergrenze von 69,5 at.\% noch bulk-glass-artiges Verhalten bei einer Kühlrate von $10^{3} \mathrm{~K} /$ s gefunden. Diese Grenze entspricht der Beobachtung in dieser Arbeit, dass etwa 70 at.\%. die kritische Konzentration darstellt. Die Zusammensetzung der dünnsten Schicht von nur $10 \mathrm{~nm}$ kann mit EDX nicht sinnvoll bestimmt werden. Die Messung an der vorherigen Referenzschicht, zusammen mit einer leichten Anhebung der Palladiumrate, deuten aber auf eine korrekte Zusammensetzung hin. Sowohl das geringe Schermodul bei Raumtemperatur (20-30 GPa), als auch die Röntgenweitwinkelmessung (abgebildet im Anhang im Abb. A.1) liefern ebenfalls keine Hinweise auf Kristallinität dieser Schicht.

Besonders wichtig ist eine gleichbleibende Zusammensetzung der Schichten. Der einzige Parameter, der bei den Proben systematisch variiert werden soll, ist die Schichtdicke. Nur wenn alle anderen Parameter dabei konstant gehalten werden, ist eine solche Probenserie aussagekräftig.

Wie in Tabelle 4.1 aufgelistet, ist diese Anforderung gut erfüllt. Die Doppelpaddelschichten weisen, mit Ausnahme der nicht gut bekannten $10 \mathrm{~nm}$ Schicht, alle sehr ähnliche Zusammensetzungen auf, mit Variationen, die bei der Probenherstellung durch Aufdampfen mit Elektronenstrahlverdampfern bei hohen Raten zu erwarten sind.

\subsubsection{Rauigkeiten und Morphologie}

In dieser Arbeit werden mechanische Messungen an Schichten vorgestellt, die eine Längenskala im Bereich von Nanometern aufweisen. Wie bei allen nanoskaligen Materialien sind größere Einflüsse der Oberflächen bzw. Grenzflächen als bei massiven Proben zu erwarten. 


\section{Tantal-Zwischenschicht:}

Die Grenzfläche der Filme zum Substrat ist durch die Oberfläche der Tantalschicht auf den Silizium-Paddeln gegeben. Diese Schicht verhindert mit einer Dicke von $50 \mathrm{~nm}$ das Eindiffundieren von z. B. Kupfer in das Silizium [71, 72]. Auch wenn diese gesputterten Schichten i. A. glatt aufwachsen [68], so ist damit dennoch eine Erhöhung der Rauigkeit gegenüber den polierten Siliziumoberflächen gegeben. Wie in Kapitel 4.1.3 gezeigt, besteht die TaOberfläche aus Körnern mit 5-10 nm Durchmesser. Es ergeben sich typische Höhenunterschiede von bis zu $5 \mathrm{~nm}$, die für den dünnsten amorphen Film die Geometrie bereits deutlich gegenüber einem flachen, glatten Film verändern. Durch den hohen Schmelzpunkt von Tantal ist nicht zu erwarten, dass diese Strukturen beim Heizen bis etwa $700 \mathrm{~K}$ nennenswert anwachsen und auch dickere Filme beeinflussen. Für die dickeren Filme wird im Gegenteil erwartet, dass eine Glättung der Oberfläche auftritt, wie an Multilagen von amorphen und kristallinen Schichten bereits gezeigt wurde [73, 74].

\section{Kristallisierte Schichten als Unterlage:}

Kristallisierte PdCuSi-Schichten können prinzipiell als Unterlage für amorphe Schichten verwendet werden. Dazu werden die Paddel bei $730 \mathrm{~K}$ für $30 \mathrm{Minu}-$ ten ausgelagert, um die Kristallisation und Kornvergröberung abzuschließen. Mechanisch erweist sich diese „Unterlage“ danach stabil, aufeinanderfolgende Heizzyklen zeigen gleiche Dämpfungsverläufe. Die STM-Aufnahme einer so kristallisierten PdCuSi-Schicht (Abb. 4.6) zeigt jedoch eine hohe Rauigkeit, der Film erscheint bis zum Substrat aufgerissen. Für die, in der Arbeit von P. Rösner [6] studierten, 300-600 nm dicken ZrAlCu-Filme treten dadurch keine Unterschiede in den mechanischen Messungen auf. Für den Bereich um $50 \mathrm{~nm}$ in dieser Arbeit ändert sich das Spektrum deutlich. Der Vergleich der jeweiligen Messungen in den Abbildungen 4.13 und 4.22 zeigt trotz fast gleicher Schichtdicke und -zusammensetzung sehr verschiedenes Verhalten. Der auf dem glatten Substrat deponierte Film zeigt bei tiefen Temperaturen deutliche Dämpfung, wohingegen der Film auf der rauen Unterlage hier kaum messbaren Verlust aufweist. Auch bei höherer Temperatur differiert das Spektrum. Die Diskussion über die möglichen Ursachen dieses deutlichen Effektes erfolgt in Abschnitt 5.5.1.

Auf der rau hergestellten, kristallisierten, $50 \mathrm{~nm}$ dicken Schicht wurden weiterhin Filme mit 100 und 25 nm Dicke vermessen. Beide Spektren werden im Anhang A.2 gezeigt und diskustiert. Eine sichere Interpretation dieser Messungen ist schwierig, da die genaue Rauigkeit der Multilagen nicht ohne Entnehmen aus der Vakuumkammer bestimmt werden konnte, zudem zeigen sich bei dieser Geometrie weitere Probleme: Die Messungen deuten auf frühzeitige Kristallisation hin, was durch heterogene Keimbildung erklärt werden kann [75], für die oberste Schicht der Multilagen ist sogar ein Aufreißen der 
Schicht beim Aufheizen nicht auszuschließen. Mit Kenntnis dieser Komplikationen sind daraufhin amorphe dünne Filme nur auf jeweils neue DPO aufgebracht worden.

\section{Auf glatten Substraten:}

Das Wachstum dünner amorpher Schichten beim Aufdampfen auf glatten Substraten wurde am System ZrAlCu von S. G. Mayr untersucht [76]. Demnach wachsen amorphe dünne Schichten anfänglich in Form von kleinen Inseln, die im Weiteren koaleszieren. Der Übergang erfolgt auf atomar glattem HOPG (highly oriented pyrolytic graphite) bei etwa $5 \mathrm{~nm}$ [77]. Der geschlossene Film weist eine sehr geringe Rauigkeit auf, die beim weiteren Aufdampfen nur sehr langsam ansteigt [76].

Die Frage, ob topographische Änderungen in der Schichtdickenserie auf glatten Substraten für mechanische Unterschiede verantwortlich sein können, muss also gestellt werden. Die dünnste spektroskopierte Schicht von $10 \mathrm{~nm}$ zeigt sich auf einem glatten Referenzsubstrat geschlossen (Abb. 4.7). Es gibt keine Hinweise auf nicht zusammenhängende Inseln oder Risse.

Damit kann geschlossen werden, dass auch die dünnste Schichtdicke bereits über dem Frühstadium der Inselkoaleszens liegt und keine großen Änderungen in der Rauigkeit zu erwarten sind. Diese Analyse wird auch von den Kleinwinkel-Röntgenmessungen unterstützt: Für alle Schichten von $100 \mathrm{~nm}$ und darunter ergeben sich in den Reflektivitätskurven periodische Maxima, die mit einem Modell geschlossener Schichten angepasst werden können. Die Oberflächenrauigkeiten der Schichten, bestimmt aus einer solchen Anpassung, ergeben für die dünnsten Schichten vergleichbare Werte und zu den dickeren Schichten den erwarteten Anstieg (siehe auch Tabelle 4.2). Zusammenfassend ergeben alle Messungen keinen Hinweis auf topologische Unterschiede in den Schichten, die Einfluss auf mechanische Eigenschaften nehmen könnten.

\subsection{Genauigkeit der mechanischen Messungen und unerwünschte Verlustprozesse}

Eine methodische Diskussion der Messgenauigkeit der hier verwendeten Technik ist bereits in Kapitel 2.4.4 erfolgt, an dieser Stelle soll der Einfluss von Fehlerquellen auf die Messergebnisse analysiert werden. Die ab Abschnitt 4.2.1 gezeigten Spektren der mechanischen Messgrößen Resonanzfrequenz und inverse Güte weisen ein sehr gutes Signal-zu-Rausch-Verhältnis auf. Die Streuung der Messwerte liegt maximal im einstelligen Prozentbereich, damit ergeben sich glatte Verläufe der Messpunkte. Die Messgenau- 
igkeit stellt sich, wie bereits in Abschnitt 2.4.4 abgeschätzt, auch für sehr dünne Schichten von deutlich unter $100 \mathrm{~nm}$ als geeignet heraus. Im Messbereich tiefster Temperaturen von unter $10 \mathrm{~K}$ wurde dies bereits durch Messungen an amorphen $\mathrm{SiO}_{2}$-Schichten gezeigt [78], bei dem hier studierten Temperaturbereich von 200-700 K sind die Gegebenheiten jedoch anders. Die Vermessung der Schichtdämpfung, durch Differenz von Leermessung zu bedampfter Messung, liefert auch für die dünnsten Schichten von 25 bzw. $10 \mathrm{~nm}$ noch signifikante Werte. Dünnere Filme sind nicht vermessen worden, da sich bereits für den $10 \mathrm{~nm}$ dicken Film eine präparative Grenze zeigte ${ }^{1}$.

\section{Dämpfungsmaxima:}

Die Mehrzahl der gemessenen Dämpfungsverläufe sind nicht frei von Störungen, die sich in scharfen Dämpfungsmaxima bei verschiedenen Temperaturen darstellen. Diese Maxima sind ein methodisches Problem und treten sowohl in den Leermessungen der Paddel selbst, als auch mit amorphen Filmen beschichtet auf. Es kann daher ausgeschlossen werden, dass diesen Dämpfungsmaxima Relaxationen in den amorphen Schichten entsprechen. Ein solches Maximum ist zumeist scharf begrenzt auf einige Grad in der Temperaturauftragung, sehr ausgeprägte Maxima können aber auch einen Bereich bis zu 30 K stark stören. Die Temperaturen bei denen diese Peaks auftreten variieren im Wesentlichen von Paddel zu Paddel, Verschiebungen ergeben sich aber auch von Messdurchlauf zu Messdurchlauf. Sie treten beim Aufheizen und beim Abkühlen auf.

Diese Maxima führen zu keinem Einfluss auf den Dämpfungsverlauf an sich, wie mehrfache Leermessungen mit Maxima bei verschiedenen Temperaturen zeigen. Mit Ausnahme der direkt gestörten Temperaturbereiche ist der Verlauf exakt reproduzierbar. Messungen der amorphen dünnen Schichten sind damit auch bei Auftreten solcher Peaks, nur durch die Messungenauigkeit beschränkt, korrekt.

Im Folgenden sollen mögliche Mechanismen der unerwünschten Prozesse vorgestellt werden:

1. Einen sehr naheliegenden Verlust stellt die Dissipation von mechanischer Energie über die Klemmung des Oszillators dar. Die Deformation des DPOs ist bei der verwendeten AS2-Mode sehr gering [79], dennoch führt ein nicht absolut fester Halt dort zu Reibungsverlusten. Eine „trockene“ Klemmung, wie sie im Ultrahochvakuum verwendet werden muss, ist dafür besonders anfällig [58]. Die These, dass die

\footnotetext{
${ }^{1}$ In diesem Fall steigt der Einfluss der Grenzfläche, diskutiert in Abschnitt 5.1.2 und Abschnitt 5.5.3
} 
Klemmung die Dämpfung verursacht, ist besonders bei dem Maximum in Abbildung 4.10 gegeben, da dieses stets um Raumtemperatur (bei unverspannter Klemmung) auftritt.

2. Weiterhin stellt die Absorption oder Desorption von Gaskontaminationen einen möglichen Verlustprozess dar. Ein Wasserfilm und auch weiteres in der UHV Anlage vorhandenes Wasser werden durch den Ausheizvorgang weitgehend entfernt, verbleibende Restgase sind CO und $\mathrm{H}_{2}$. Die Analyse mit einem Quadrupol-Massenspektrometer zeigt, dass diese Gase mit einer N2 Kühlfalle und einer Titan-Sublimationspumpe kurzfristig weiter entfernt werden können. Sowohl das Element Tantal der Zwischenschicht, als auch das Element Palladium der amorphen Schicht, weisen eine starke Affinität zu Wasserstoff auf [80]. Damit ist das Beladen mit Wasserstoff bei niedriger Temperatur und die Abgabe bei hoher Temperatur ein plausibler Prozess. Die enge Verteilung der Maxima ist mit diesem Prozess aber nicht vereinbar.

3. Die ungefähre Position bestimmter Peaks im Dämpfungsverlauf ist charakteristisch jeweils für eine Charge von Paddeln, die aus den selben Silizium-Wafern hergestellt worden sind. Mit dieser Beobachtung ist zu unterstellen, dass diese Wafer Verunreinigungen von Fremdatomen aufweisen, die wie bei einem Snook-Effekt [75, 45] zwischen verschiedenen Positionen im Kristallgitter springen können. Die These von charakteristischen Verunreinigungen kann zum einen die schmale Form der Maxima (einfacher, thermisch aktivierter Prozess) und zum anderen auch die hohe Relaxationsstärke erklären (Beispiele in [45]).

Auch der Laserschneideprozess der Paddel führt, wie bereits in [6] aufgezeigt, nachträglich zu erhöhter Dämpfung. Die Schnittkanten, wie in Abbildung 3.8 gezeigt, bestehen aus stark geschädigtem Material, in dem weitere Dämpfungsprozesse wie Korngrenzendämpfung zu erwarten sind. In [12] wird gezeigt, dass der Dämpfungsverlauf, der mit einem Ätzverfahren hergestellten Doppelpaddel, mit dem thermoelastischen Effekt zu beschreiben ist. Für die hier verwendeten Paddel trifft dies nur auf den Tieftemperaturbereich zu, bei höheren Temperaturen steigt die Dämpfung mindestens wie $Q^{-1} \propto\left(T-T_{o}\right)^{3}$ an. Auch dieser Anstieg kann z. B auf kristalline Defekte zurückgeführt werden. 


\subsection{Diskussion neuer Resultate zu fragilen me- tallischen Gläsern}

Die Messung des Relaxationsspektrums von PdCuSi-Filmen schließt eine Lücke in den mechanischen Analysen metallischer Gläser in Bezug auf sekundäre Relaxationen. Bisher wurde die Legierung ZrAlCu als Vertreter der Gruppe der starken Gläser in Form dünner Schichten [81] als auch als massive Bänder [82] vermessen. Ein Vertreter der fragilen Gläser, PdCuSi, wurde in Form massiver Bänder ebenfalls bezüglich sekundärer Relaxationen charakterisiert [83]. An dieser Stelle werden die neuen Ergebnisse an PdCuSiSchichten mit diesen Resultaten in Bezug gesetzt und damit neue Erkenntnisse vorgestellt. Dabei werden zunächst nur die dickeren Schichten $(\mathrm{d}>50 \mathrm{~nm})$ berücksichtigt. Effekte durch die Probengröße selbst werden in Kapitel 5.5 diskutiert.

\subsubsection{Vergleich mit PdCuSi-Bändern}

Das Relaxationsspektrum von PdCuSi wurde von J. Hachenberg mit einem Dynamisch Mechanischen Analysator (DMA) ${ }^{2}$ mittels dünner Bänder bestimmt. Diese Bänder werden durch ein melt-spinning-Verfahren hergestellt, mit einer charakteristischen Kühlrate von $10^{6} \mathrm{~K} / \mathrm{s}$ [3].

Das Verlustspektrum, gemessen mit einer Heizrate von $5 \mathrm{~K} / \mathrm{min}$ mit der Spektroskopiefrequenz von $1 \mathrm{~Hz}$, ist in Abbildung 5.1 gezeigt. Dabei ist eine deutliche Trennung von $\beta$-Prozess und $\alpha$-Prozess durch die Steigungsänderung bei $650 \mathrm{~K}$ erkennbar. Der $\alpha$-Prozess wird wegen einsetzender Kristallisation (bei dem Wendepunkt in $\mathrm{E}^{\prime \prime}$, bei $660 \mathrm{~K}$ ) nicht vollständig aufgezeichnet. Unterhalb von $620 \mathrm{~K}$ wird ein konstanter Verlust, als NCL- bzw. sub $T_{g^{-}}$ Anteil bezeichnet, gemessen.

Der Vergleich einer Messung mit einer 200 nm dicken PdCuSi-Schicht, spektroskopiert mit dem wesentlich empfindlicheren Doppelpaddel-Oszillator, dargestellt in der Abbildung 5.2, zeigt einige Unterschiede auf: Insbesondere stellt sich der Verlauf des Verlustmodules $G^{\prime \prime}$ in Abbildung 4.17 unterhalb von $620 \mathrm{~K}$ nicht als konstant dar. Bereits oberhalb von Raumtemperatur ist eine Abnahme des Verlustes zu beobachten, gefolgt von einer kontinuierlichen Zunahme ab $440 \mathrm{~K}$ bis zu der Temperatur, bei der einsetzende Kristallisation zu einer schlagartigen Abnahme der Dämpfung führt, hier etwa $640 \mathrm{~K}$.

Um diese Diskrepanz für den selben Glasbildner zu erklären, gibt es zwei mögliche Deutungen: Zum einen kann der Unterschied durch die Messmethode bedingt sein. In diesem Fall stellt der gerade Verlauf bis $620 \mathrm{~K}$ bei

${ }^{2}$ Die Technik ist beschrieben im Abschnitt 2.3. 


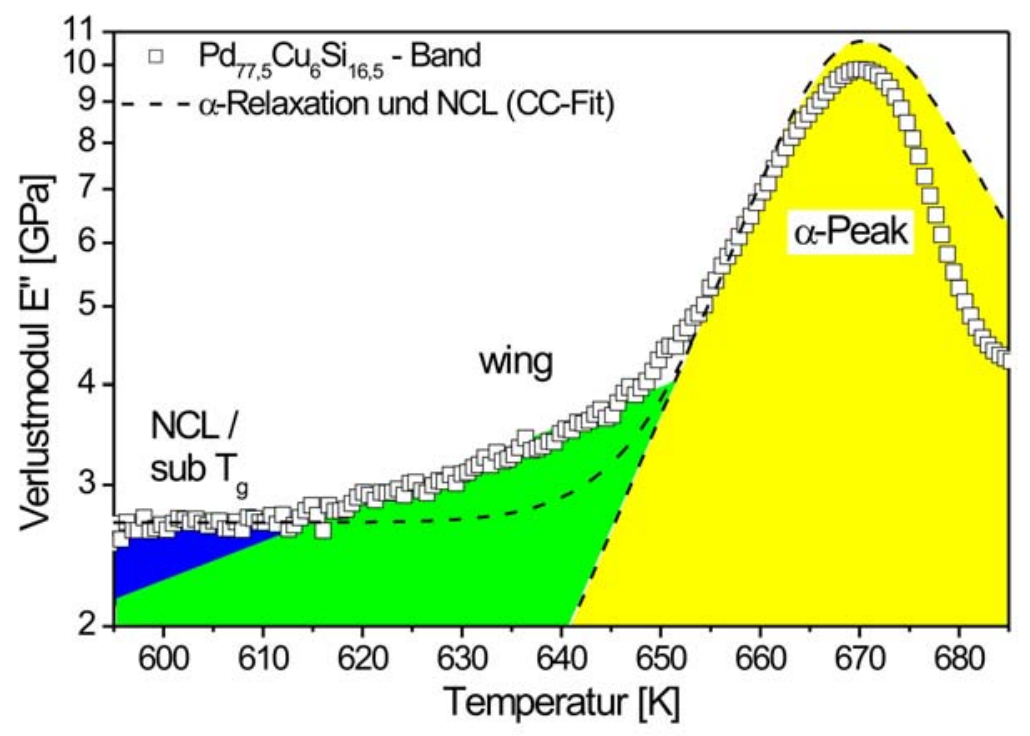

Abbildung 5.1: Relaxationsspektrum von PdCuSi, gemessen mittels DMA von J. Hachenberg [7]. Der $\beta$-Prozess tritt deutlich vom $\alpha$-Prozess getrennt auf.

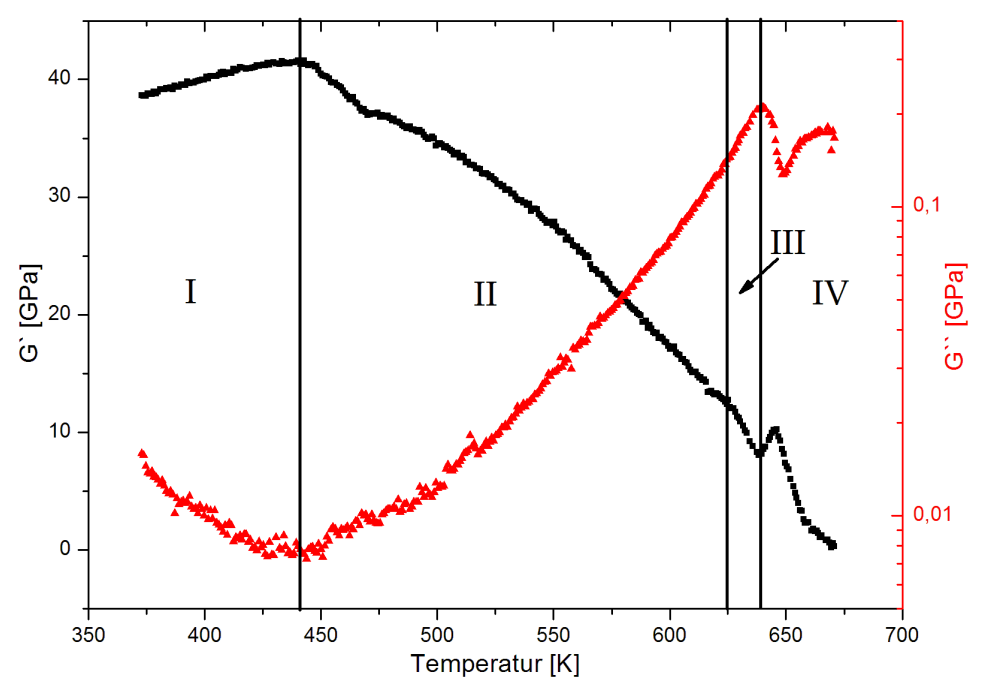

Abbildung 5.2: Komplexer Schermodul der $200 \mathrm{~nm}$ dicken PdCuSi-Schicht, wie in Abbildung 4.17. Der gemessene Temperaturbereich kann in vier Bereiche aufgeteilt werden. Bereich I: Relaxation über $\beta$-Alterung, Bereich II: Ansteigender $\beta$-Velust (wing), Bereich III: Schnelles Erweichen der Schicht mit dem einsetzenden $\alpha$-Prozess, Bereich IV: Einsetztende Kristallisation. 
der Messung mit dem DMA die untere Grenze der Messgenauigkeit dar. Das Doppelpaddel reagiert hingegen auf sehr kleine Dämpfungseinflüsse und ist dadurch besser geeignet bei tieferen Temperaturen den Verlust in der Probe zu messen.

Die andere Erklärungsmöglichkeit ist, dass Unterschiede in der Probenherstellung diesen Unterschied im Verlustspektrum bedingen. Man kann dabei annehmen, dass die effektive Abkühlrate beim Aufdampfen wesentlich höher als beim melt-spinning-Verfahren ist und damit die Probe weniger gut relaxiert ist. In einer solchen weniger gut gepackten Anordnung der Atome kann die Anregungsenergie für Verlustprozesse reduziert sein. Damit wäre das Auftreten von Relaxationsprozessen bei tieferen Temperaturen gegenüber Bändern erklärbar.

Das zweite Argument stellt sicherlich einen wichtigen Aspekt bei der Interpretation des Spektrums dar, es wird im Folgenden bei der Diskussion der Alterungsprozesse noch einmal verwendet. Für die erste Interpretationsmöglichkeit spricht aber, dass Messungen an amorphen PdCuSi-Bändern mit einer anderen Methode ${ }^{3}$ bei Raumtemperatur deutlich niedrigere Verluste als in Abbildung 5.1 ausweisen. Auch die Messungen von P. Rösner an $\mathrm{ZrAlCu}-$ Schichten mittels DPO-Spektroskopie zeigen einen kontinuierlichen Anstieg des Verlustes über einen weiten Temperaturbereich, zudem treten hier ebenfalls wesentlich kleinere Verluste auf [6].

Im Falle der Messung von PdCuSi in Form dünner Schichten zeigt sich die Trennung von $\beta$ - und $\alpha$-Prozess oberhalb von $600 \mathrm{~K}$ wesentlich schwieriger als bei DMA-Messungen an Bändern. Die Bestimmung des Realteils des Schermoduls (Abb. 5.2) aus der Frequenzverschiebung zeigt bei etwa $625 \mathrm{~K}$ das Erweichen des Glases an, die Steigung des Verlustmodules ändert sich bei dieser Temperatur jedoch nur sehr gering. Das deutliche Erweichen der Probe bei dieser Temperatur weist auf einen $\alpha$-Prozess hin, der von dem $\beta$-Prozess aufgrund einer deutlichen Überlappung nicht getrennt ist. Das Zusammenlaufen von $\alpha$ und $\beta$-Prozess bei hohen Temperaturen wird als merging bezeichnet und ist für organische Glasbildner bereits bekannt. Wie in Abbildung 1.5 für o-Terphenyl dargestellt, bzw. auch in Abbildung 1.2 illustriert, verschieben sich die Relaxationszeiten von $\alpha$-Prozess und $\beta$-Prozess verschieden stark mit der Temperatur. Auch für metallische Gläser konnte dieser Effekt eindeutig gezeigt werden [31]. Je höher die Spektroskopiefrequenz oder Heizrate, desto näher rücken damit diese Prozesse in einer Temperaturauftragung zusammen $[4,31]$.

\footnotetext{
${ }^{3}$ In Zusammenarbeit mit Prof. R. Richert wurden im Juli 2007 Messungen u. a. an PdCuSi-Bändern in Form resonant angeregter Biegebalken durchgeführt. Die Messmethode ist in [84] vorgestellt.
} 
Die Glastemperatur der $200 \mathrm{~nm}$ dicken Schicht beim Heizen mit $1 \mathrm{~K} / \mathrm{min}$ kann durch das rasche Abfallen des Schermoduls zu $625 \mathrm{~K}$ bestimmt werden. Vergleicht man diese Angabe mit Messungen an PdCuSi-Bändern, so stimmt diese Temperatur mit der Glastemperatur von kalorimetrischen Messungen ${ }^{4}$ überein [7]. Beim Heizen mit $5 \mathrm{~K} / \mathrm{min}$ wurde in [7] die Glastemperatur als Onset im Wärmefluss (zur unterkühlten Schmelze) zu $626 \mathrm{~K}$ bestimmt. Das mechanische Erweichen der PdCuSi-Bänder, bzw. das starke Ansteigen der Dämpfung, bei $1 \mathrm{~Hz}$ Spektroskopiefrequenz wird in der zitierten Arbeit jedoch erst bei etwa 655-660 K gemessen. Bevor diese Unterschiede physikalisch interpretiert werden, soll kurz auf die Problematik der Vergleichbarkeit der Temperaturkalibrierungen eingegangen werden. Sowohl beim DoppelpaddelOszillator, bei der DSC- (differential scanning calorimeter), als auch bei der DMA-Temperaturbestimmung wird eine außerhalb der Probe gemessene Temperatur mit einer geeigneten Kalibrierung auf die Probentemperatur umgerechnet. Der Fehler dieser Kalibrierungen ist schwer abzuschätzen, für die DPO-Messung wurde das hier diskutierte Paddel DPO_DB_05 direkt nach der Messung kalibriert (beschrieben in Abschnitt 3.1.1), jedoch kann auch dabei die Temperatur leicht verfälscht werden. Die Temperaturkalibrierung der DSC ist über die Schmelzpunktbestimmung von Indium und Zink realisiert und ist damit besonders präzise. Der Fehler in der DMATemperaturbestimmung kann hier nicht abgeschätzt werden, es gibt aber neuere Messungen mit dieser Apparatur, in denen die Glastemperatur von PdCuSi-Bändern bei einer Heizrate von $1,25 \mathrm{~K} / \mathrm{min}$ zu $630 \mathrm{~K}$ bestimmt wird [85]. Damit ist es plausibel, dass die Abweichungen bezüglich der Glastemperatur im Wesentlichen durch verschiedene Kalibrierungen zu erklären sind, es besteht jedoch auch die Möglichkeit einer physikalischen Deutung dieser Unterschiede.

Zunächst ist die Glastemperatur, wie in Kapitel 1.2.1 beschrieben, heizratenund frequenzabhängig. Die wesentlich höhere Frequenz der DoppelpaddelMessung lässt, gegenüber der niederfrequenten DMA-Messung, eine höhere Glastemperatur erwarten [4]. Auch die höhere Abkühlrate führt zu der Erwartung einer erhöhten Glastemperatur. Die Tatsache, dass dieser Effekt nicht beobachtet wird, führt zu dem Schluss, dass es, wenn überhaupt, die Eigenschaften des dünnen Films sind, die eine herabgesetzte Glastemperatur bedingen. Ein bei Raumtemperatur aufgedampfter Film kann, wie bereits zuvor angedeutet, weniger gut gepackt sein. Zudem kann die Oberfläche, durch die Geometrie bedingt, die Eigenschaften beeinflussen. In einer Studie von S. Vauth und S. G. Mayr wurde stark erhöhte Mobilität von Atomen nahe einer Oberfläche in Simulationen gezeigt [86]. Damit kann die Glastempera-

${ }^{4}$ Diese Messungen wurden mit einem „Perkin Elmer DSC 7“ durchgeführt. 
tur einer dünnen Schicht prinzipiell tiefer liegen als im bulk. Für die $200 \mathrm{~nm}$ dicke Schicht wird jedoch noch keine Schichtdickenabhängigkeit erwartet ${ }^{5}$.

Die Auftragung des komplexen Schermoduls von PdCuSi in Abbildung 4.17 zeigt eine eindeutige Korrelation von Imaginärteil und Realteil. Nach der Kramers-Kronig-Relation sind der Realteil und Imaginärteil einer komplexen Größe über Integration verknüpft [87]. In [7] wurde dies bereits für den Bereich des Glasübergangs gezeigt. Erst in der vorliegenden Arbeit zeigt sich diese Kopplung über den gesamten Temperaturbereich, was die hohe Messgenauigkeit der DPO-Methode unterstreicht. Diese präzise Vermessung bei Temperaturen weit unter $T_{g}$ wird auch durch die Fragilität des Glases begünstigt, da diese zu einem besonders ausgeprägten $\beta$-Prozess führt [7].

\subsubsection{Vergleich mit dem starken Glas $\mathrm{ZrAlCu}$}

Für den Vergleich zu Messungen an einem starken Glas, von P. Rösner mit einem Doppelpaddel spektroskopiert, ist in Abbildung 5.3 der komplexe Modul einer $300 \mathrm{~nm}$ dicken Schicht ZrAlCu gezeigt. Bei dieser Messung wurde schrittweise und nicht kontinuierlich geheizt, es ergibt sich eine effektive Heizrate von ca. $0,2 \mathrm{~K} / \mathrm{min}$.

Der Vergleich mit Abbildung 4.17 zeigt, dass der Verlauf der komplexen Module hier recht ähnlich ausfällt. Insbesondere der Wertebereich des Verlustes ist in sehr guter Übereinstimmung. Man erkennt bei ZrAlCu ebenfalls anfänglich eine Abnahme des Verlustes, gefolgt von einer kontinuierlichen Zunahme ab 450 K. Ab 620 K steigt der Verlust allmählich steiler an. Der Realteil des Schermoduls fällt kontinuierlich von $30 \mathrm{GPa}$ auf etwa $10 \mathrm{GPa}$ ab. Die Kristallisation der Schicht tritt für diese Legierung und Spektroskopiefrequenz noch vor der $\alpha$-Relaxation auf [6]. Dies zeigt sich am deutlichsten beim Vergleich der reellen Schermodule $\mathrm{G}^{\prime}$ : Bei PdCuSi fällt dieser Modul ab $625 \mathrm{~K}$ sehr schnell auf nahezu Null, während beim $\mathrm{ZrAlCu}$ ein nahezu konstanter Wert erreicht wird. In [81] wird der Verlust ab $620 \mathrm{~K}$ als wing bezeichnet, der ansteigende Verlust unterhalb als $s u b-T_{g}$. Eine solche Unterteilung ist im Verlustspektrum von PdCuSi nicht zu identifizieren. Eine mehr oder weniger deutliche Steigungsänderung im Verlust tritt nur beim Erweichen der Schicht bei $625 \mathrm{~K}$ auf. Sowohl für PdCuSi- als auch für $\mathrm{ZrAlCu}$ ist der Temperaturverlauf des Verlustmodules in weiten Teilen als positiv gekrümmt zu beschreiben.

\footnotetext{
${ }^{5}$ Der Aspekt der Schichtdickenabhängigkeit der Glastemperatur wird detaillierter in Abschnitt 5.5.4 und 5.5.6 besprochen.
} 


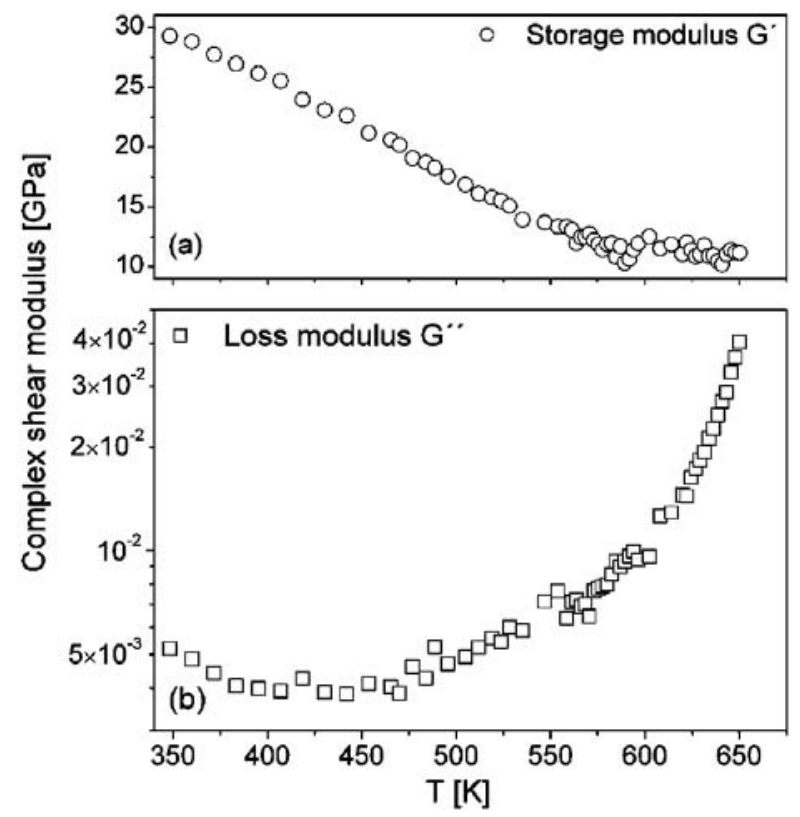

Abbildung 5.3: Bestimmung des komplexen Schermoduls einer $300 \mathrm{~nm}$ dicken ZrAlCu-Schicht, entnommen aus [81].

\subsubsection{Interpretation im Rahmen eines Potentielle-Energie-Landschafts-Modells}

Das Potentielle-Energie-Landschaft (PEL)-Modell stellt eine sehr einfache Darstellung mikroskopischer Konfigurationsmöglichkeiten dar. Dabei ist von einem komplexen System, bestehend aus $\mathrm{N}$ Teilchen, der $3 \cdot \mathrm{N}$ dimensionale Zustand mit nur einer generalisierten Koordinate repräsentiert. Die potentielle Energie des Gesamtsystems wird nun als Funktion dieser Konfigurationskoordinate aufgetragen. Eine schematische Darstellung einer solchen "Landschaft" ist in Abbildung 5.4 dargestellt. Von P. G. Debenedetti und F. H. Stillinger stammt der Vorschlag, dass ein großer Temperaturbereich bei Gläsern durch diese Energielandschaft beschreibbar ist, bzw. in der Beschreibung der Dynamik berücksichtigt werden muss [13].

In einer solchen Energie-Landschaft gibt es eine Vielzahl von breiten Minima (megabasins), die günstige Konfigurationen des Systems darstellen. Der Übergang von einem breiten Minimum in ein anderes ist über eine $\alpha$-Relaxation möglich. Die Energiebarriere kann sowohl thermisch (nahe $T_{g}$ ) oder auch durch von außen angelegte mechanische Spannung überwunden werden. Das cooperative shear model (CSM) beschreibt die Reduzierung der Barrierenhöhe durch eine mechanische Scherspannung und liefert quantitative Vorhersagen 


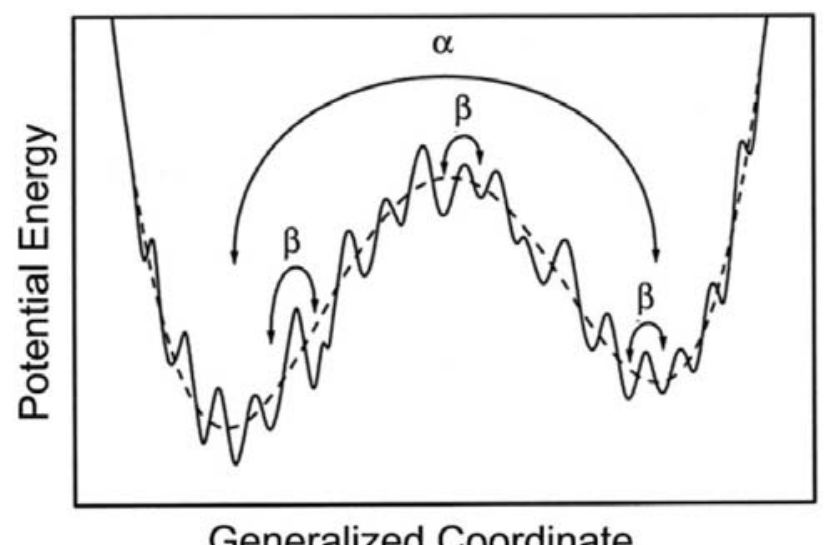

Abbildung 5.4: Schematische Darstellung der PEL, aus [83] nach [13]. Die viskose $\alpha$-Relaxation entspricht Übergängen zwischen sogenannten megabasins und die $\beta$-Relaxation einem hopping zwischen den Sub-Minima

für die kritische Scherspannung, bei der das Glas zu fließen beginnt [39]. Die megabasins weisen nach Debenedetti auch eine Unterstruktur von SubMinima auf. Übergänge zwischen diesen Sub-Minima stellen in diesem Bild die $\beta$-Relaxation dar.

Das PEL-Modell eignet sich gut zur Modellierung mechanischer Messungen, da die Krümmung des Potentials das Schermodul beeinflusst ${ }^{6}$. Sowohl Simulationen als auch Experimente zeigen einen einfachen linearen Zusammenhang zwischen Schermodul und Konfigurationsenergie [88, 89].

Zunächst ohne Berücksichtigung mikroskopischer Betrachtungen sollen die Verlustspektren bzw. die Temperaturabhängigkeiten der Schermodule in dem Temperaturbereich von Raumtemperatur bis zum Glasübergang in diesem Modell diskutiert werden.

Das Herstellen durch Aufdampfen auf ein Substrat bei Raumtemperatur bedingt eine sehr hohe Abkühlrate. Damit wird eine bei Raumtemperatur ungünstige Konfiguration, hoch in der Energielandschaft, eingenommen. Bereits bei moderatem Heizen kann die Schicht relaxieren (aging), das Schermodul steigt dabei an, die Dämpfung nimmt ab. Die $\alpha$-Relaxation kommt hier als möglicher Prozess nicht in Betracht, da in diesem Temperaturbereich die strukturellen Relaxationszeiten astronomisch ausfallen, beachtet man die schnelle Divergenz bzw. das überexponentielle Anwachsen dieser

\footnotetext{
${ }^{6}$ Mit einem Ansatz nach Frenkel für die Energiedichte $\Phi$ für ein STZ als Funktion der Scherdehnung $\gamma$ mit $\Phi / \Phi_{0}=\sin ^{2}\left(\pi \gamma / 4 \gamma_{c}\right)$ gilt: $G=\left(\partial^{2} \Phi / \partial \gamma^{2}\right)_{\gamma=0}$ [39]. Dabei bezeichnet $\gamma_{c}$ die kritische Scherspannung.
} 
Zeitskala unter $T_{g}$ (siehe Kapitel 1.3.2). Damit bleibt der $\beta$-Prozess als Pfad der Relaxation, im Bild der PEL ein hopping innerhalb des megabasins über Sub-Minima nach „unten“. Die Zeitkonstante dieser Relaxation ist nicht gut bekannt, sie könnte aber neben der Temperatur auch empfindlich von der Stöchiometrie, Dichte und auch von der Lage in der potentiellen Energie selbst abhängen. Aus Alterungsexperimenten bei Temperaturen von RT bis nahe $T_{g}$ an Proben aus massivem PdNiP [34] kann abgeschätzt werden, dass Zeitkonstanten von Stunden bis einigen Tagen hier relevant sind. Bei steigender Temperatur werden thermisch getriebene Prozesse mit höherer Energie angeregt bis, etwa bei der Glastemperatur, die Probe thermisch aktiviert über einen $\alpha$-Prozess fließen kann. Wegen der im Vergleich zur Zeitkonstante des $\beta$-Prozesses schnellen Temperaturerhöhung der Probe mit 0,2-1 K/min tritt keine Sättigung des Alterungsprozesses ein. Das Schermodul steigt also nur so lange (in der Zeit) bis eine Konfiguration vorliegt, die der aktuellen Temperatur entspricht. Da die Temperatur kontinuierlich steigt, führt dies zu einer Abnahme des Schermoduls in Abbildung 4.17 oberhalb dieser Temperatur, gleichzeitig steigt die Dämpfung wieder an. Auch dieses Ansteigen in der PEL verläuft nun über $\beta$-Prozesse, damit kann der gesamte Anstieg der Dämpfung, bei PdCuSi ab $440 \mathrm{~K}$, als wing bezeichnet werden. Sobald die Temperatur hoch genug ist, um $\alpha$-Prozesse auf der Zeitskala der Spektroskopiefrequenz zu ermöglichen ${ }^{7}$, tritt dieser Prozess als weiterer Anstieg im mechanischen Verlust auf. Da das System oberhalb der Glastemperatur einen großen Teil des Phasenraums abtasten kann [90], tritt für metallische Gläser nach endlicher Zeit immer Kristallisation auf. Diese entspricht einem scharfen, tiefen Minimum in der PEL. Die Differenz in der Konfigurationsenergie wird über Kristallisationswärme abgegeben.

\subsection{Relaxationen in Gläsern}

In der vorliegenden Arbeit wurde der messbare Temperaturbereich des Doppelpaddel-Messaufbaus mit einer Kühlmöglichkeit mit flüssigem Stickstoff erweitert. Damit kann auch das Verlustspektrum im Temperaturbereich unter Raumtemperatur aufgenommen werden, um den wing weiterzuverfolgen oder andere Prozesse zu beobachten. Diese Ergebnisse, aufgezeigt auf den Seiten 72 ff., sollen ebenfalls im Kontext weiterer aktueller Ergebnisse diskutiert werden, um das Bild des Relaxationsspektrums im Stile einer LunkenheimerLoidl-Darstellung (Abb. 1.2) für metallische Gläser zu erweitern. Dabei gestaltet sich eine Diskussion der Abhängigkeiten der Prozesse von der Tempe-

\footnotetext{
${ }^{7}$ Spontane $\alpha$-Prozesse sind aufgrund der Boltzmann Verteilung der Energie auch bei tieferen Temperaturen möglich, aber entsprechend selten.
} 
ratur, methodisch bedingt, einfacher als eine reine Frequenzabhängigkeit. Wie im vorherigen Abschnitt beginnt die Übersicht der Relaxationsprozesse bei dem $\alpha$-Prozess, der durch die Kristallisationsneigung der metallischen Gläser nur in einem schmalen Temperaturbereich auftritt. Unterhalb der Glastemperatur nimmt dieser Verlust in Abbildung 5.1 sehr schnell ab, in Übereinstimmung mit einem Vogel-Fulcher-Tammann Verhalten der Relaxationszeit. Das Auftreten des $\beta$-Prozesses wird in den hier diskutierten Ergebnissen als wing beobachtet. Dieser sekundäre Prozess erweist sich dabei als universeller Bestandteil der Dynamik metallischer Gläsern [83]. In Abbildung 5.1 zeigt sich dieser wing als gerader Abfall mit der Temperatur in dem beobachteten kleinen Temperaturintervall. Da dieser, vermutlich durch die apparative Messgrenze, nicht zu tieferen Temperaturen weiterverfolgt werden kann, ist ein Vergleich mit der Hochfrequenzflanke einer evtl. verbreiterten Debyeschen Relaxationsfunktion (vgl. Abb. 2.2) schwierig. Bei einer Auftragung des Verlustes über der Frequenz bei fester Temperatur wird eine Signatur des $\beta$-Prozesses in Form einer Debye-(Gl. (2.17)) oder HN-Funktion (Gl. (2.19)) erwartet. Nach dem Zeit-Temperatur-Superpositionsprinzip kann eine vergleichbare Auftragung gegen die Probentemperatur aufgenommen werden [6]. Für eine Probe, die sich durch die Herstellung bedingt, nicht in einem Gleichgewichtszustand befindet, kann das Zeit-Temperatur-Superpositionsprinzip nicht uneingeschränkt gültig sein, da sich der Zustand der Probe und damit auch die Art der Relaxationsprozesse selbst beim Heizen der Probe ändern. Durch das Heizen werden also nicht nur die internen Zeitskalen mit der Temperatur verschoben, sondern gleichzeitig auch die Form des Spektrums. Dieser Aspekt ist auch in [7] diskutiert und soll hier auf die komplexe Form des wings angewendet werden.

Im Bild der PEL ändert sich der Zustand der Proben beim ersten Heizen durch Alterung, gefolgt von einem thermisch aktivierten Ansteigen in der potentiellen Energie. Damit wird beim Heizen bis $T_{g}$ ein großer Energiebereich eines megabasins ausgemessen. Der gekrümmte Verlauf des Verlustes bei dem PdCuSi-Film zeigt dabei deutlich die Asymmetrie der PEL für ein fragiles Glas. Die Steigung des Verlustes steigt, in logarithmischer Darstellung, mit der Temperatur nahezu kontinuierlich an, was damit interpretiert werden kann, dass die „höheren“ $\beta$-Prozesse einfacher angeregt werden können. Auch für das starke Glas $\mathrm{ZrAlCu}$ sind in Abbildung 5.3 (mindestens) zwei verschiedene Steigungen im Verlust zu erkennen. Es bleibt dabei die Freiheit in der Interpretation, ob die Nähe zum $\alpha$-Prozes die höheren $\beta$-Moden verstärkt oder ob umgekehrt der $\alpha$-Prozess erst durch die zuvor aktivierten $\beta$-Moden stattfinden kann.

$\mathrm{Zu}$ tieferen Temperaturen friert die $\beta$-Dynamik langsam ein, bei Raumtemperatur ist quasi keine Alterung bei den hier diskutierten Gläsern einschließlich 
PdNiP zu beobachten [50]. Die Messbarkeit eines mechanischen Verlustes hängt in diesem Temperaturbereich von der thermischen Vorbehandlung ab. Bei der Messreihe zur thermischen Vorbehandlung, abgebildet auf Seite 86, ist deutlich zu erkennen, dass eine so gealterte amorphe Schicht einen sehr kleinen, auch mit dem DPO nicht mehr messbaren Verlust, aufweist. Bei frisch hergestellten Schichten kann die vorhandene Dämpfung jedoch so genau gemessen werden, dass der abnehmende Verlust beim Abkühlen unter die Herstellungstemperatur verfolgt werden kann. Für die dünneren Filme ergeben sich jedoch bereits nach einigen Stunden beim Abkühlen und Aufheizen Differenzen in der Resonanzfrequenz, die auf Gaskontaminationen zurückgeführt werden können. Das Doppelpaddel wird über Wärmeleitung zum $\mathrm{N}_{2}$-Kühler direkt gekühlt und befindet sich zudem nicht in einem Kryostaten, sondern ist von der nicht gekühlten UHV-Kammerwand umgeben. Vorhandenes Restgas (bei $10^{-9}$ mbar) kondensiert also bevorzugt auf dem kalten, flächigen DPO. Solche Kontaminationen, die sich auch in der Dämpfung niederschlagen, werden bereits beim Heizen auf etwa $20 \mathrm{~K}$ über Raumtemperatur wieder entfernt. Solche Beeinflussungen werden bei der dicksten Schicht von $200 \mathrm{~nm}$ nicht beobachtet. Die Frequenzverschiebung durch den $200 \mathrm{~nm}$ dicken Film wird in zwei Messdurchgängen an verschiedenen Tagen nahezu identisch gemessen (Abbildung 4.21). Zudem liefern die Messungen beim Kühlen und beim anschließenden Gegenheizen vergleichbare Dämpfungswerte für die Schicht. Dies zeigt zum einen die gewünschte Reproduzierbarkeit der Messwerte aber auch, dass in diesem Temperaturbereich keine Veränderungen in der Probe stattfinden. Mit den oben abgeschätzten Zeitkonstanten für eine $\beta$-Alterung ist dies auch nicht zu erwarten. Ohne eine solche Probenveränderung wird eine lineare Abnahme der Dämpfung mit der Temperatur in der logarithmischer Auftragung gemessen (Abb. 4.19). In dem hier zugänglichen Temperaturintervall bis $200 \mathrm{~K}$ wird kein Hinweis auf weitere Verlustprozesse gefunden, der Verlauf kann mit dem fortgesetzten $\beta$-Verlust identifiziert werden.

Experimente an massiven PdNiP-Proben mit Ultraschall-Spektroskopie (Methodik beschrieben in Kapitel 2.3) mit MHz-Scherwellen zeigen ebenfalls nur einen kleinen mechanischen Verlust bei Raumtemperatur [34, 50]. Beim Abkühlen dieser Proben wird dieser Verlust unmessbar klein. Die gemessene Dämpfung des Schallsignals ist im Wesentlichen auf apparative Einflüsse zurückzuführen. Mit der Ultraschallspektroskopie konnte das (reelle) Schermodul hingegen durch präzise Laufzeitmessungen bis zu $2 \mathrm{~K}$ von T. Koeppe gemessen werden [50]. Diese Abhängigkeit ist in Abbildung 5.5 dargestellt. Unter Raumtemperatur (300 K) steigt das Schermodul beim Abkühlen zunächst linear an, was einem Anderson-Grüneisen-Verhalten [92] entspricht, welches auch bei kristallinen Metallen wie Tantal beobachtet wird [50]. Bei 


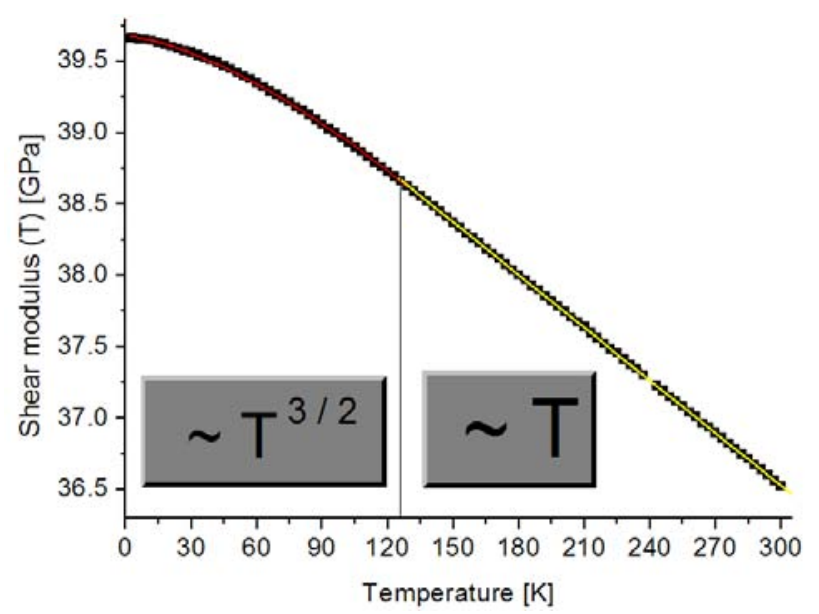

Abbildung 5.5: Temperaturabhängigkeit des Schermoduls von PdNiP, mit Ultraschallspektroskopie von T. Koeppe gemessen [50]. Abbildung entnommen aus [91].

etwa $130 \mathrm{~K}$ treten Abweichungen davon auf, das Schermodul tendiert zu einer Sättigung. Dieses Temperaturverhalten unterscheidet sich deutlich vom Verhalten kristalliner Metalle, da hier ein geringeres Krümmungsverhalten vorliegt. Man kann daraus folgern, dass für die amorphe Metallegierung andere Anregungen „ausfrieren“. Die Anpassung an die Messdaten gelingt besonders gut mit der Funktion $G(T)=G(0)-A T^{3 / 2}$, wobei $G(0)$ und $A$ freie Parameter darstellen. Eine Funktion dieser Form tritt in der Festkörperphysik bei der Magnetisierung von Ferromagneten bei tiefsten Temperaturen auf. Physikalisch wird damit die thermische Anregung von Magnonen, d. h. quantisierte Anregungen im Spinsystem eines Ferromagneten beschrieben [93]. Eine solche Funktion kann damit evtl. auch geeignet sein, andere kollektive thermische Aregungen zu beschreiben. Eine Aufklärung über die mikroskopischen Ursachen des mechanischen Verhaltens kann aus der Ultraschallmessung allein nicht erfolgen. In der hier vorliegenden Arbeit konnte der slow $\beta$-Prozess noch bis $200 \mathrm{~K}$ ohne Änderung der Aktivierung (siehe Abbildung 4.19) verfolgt werden, für die Änderung der mechanischen Eigenschaften von $2 \mathrm{~K}$ etwa $130 \mathrm{~K}$ kommen daher ,schnellere“ Prozesse in Frage. In [50] wird spekuliert, ursächlich für das beobachtete $T^{3 / 2}$-Verhalten sei die Kopplung der Atome einer erstarrten Kettenbewegung untereinander. Es ist ebenfalls möglich, dass in diesem Temperaturbereich der fast $\beta$-Prozess oder auch die zunehmende Aktivierung von soft-modes [23] beobachet wird. Oberhalb dieser Temperatur werden zunächst keine weiteren Prozesse aktiviert, das Schermodul sinkt linear nur als Folge der thermischen Ausdehnung bis, 
je nach Probenpräparation und Heizrate, etwa 200 bis $150 \mathrm{~K}$ unterhalb der Glastemperatur der einsetzende slow $\beta$-Prozess (wing) zu einer stärkeren Erweichung ${ }^{8}$ des Schermoduls führt.

Mit einem solchen Bild können die in dieser Arbeit vorgestellten Messungen an PdCuSi-Schichten in vorherige Ergebnisse zu metallischen Gläsern eingefügt werden, um damit ein genaueres Bild der Relaxationsprozesse zu erstellen.

\subsection{Einfluss der Probengröße auf das Relaxa- tionsverhalten}

Die Analyse der Verlustpektren von Proben mit eingeschränkter Geometrie stellt einen neuen Aspekt für das Verständnis der Relaxationsprozesse in metallischen Gläsern dar. Die Messergebnisse zu den immer dünneren Filmen sind im Ergebnisteil 4.2.4 ab Seite 75 aufgezeigt. An dieser Stelle sollen zuerst diese Messergebnisse vergleichend interpretiert werden, um danach Erklärungsmodelle zu den Resultaten vorzuschlagen.

\subsubsection{Dünne Filme auf rauen und glatten Substraten}

Bei einer nominellen Schichtdicke von 50 nm zeigt die Messung des temperaturabhängigen mechanischen Verlustes, dass dieser hierbei sehr stark von der Substratrauigkeit abhängt. Ein glatter Film mit $50 \mathrm{~nm}$ Ausdehnung in der Schichtdicke zeigt ein Verlustspektrum, welches sich in gleicher Weise wie der dickste Film (200 nm) darstellt: Die Messung in Abbildung 4.24 weist, wie die Messung in Abbildung 4.14, zunächst einen abnehmenden und anschließend einen immer weiter ansteigenden Verlust in Form eines breiten wings auf. Der Verlauf der Frequenzverschiebung und damit auch das reelle Schermodul verlaufen ebenfalls wie bei der $200 \mathrm{~nm}$ dicken Schicht (vgl. Abb. 4.25 u. Abb. 4.16). Damit kann die in Abschnitt 5.3.3 erfolgte Deutung in gleicher Weise auf diese Messung angewendet werden. Alle internen Prozesse verhalten sich bei dieser äußeren Längenskala nahezu unverändert. Man kann die Herstellung eines $50 \mathrm{~nm}$ dicken Films auf ein raues Substrat als eine weitere geometrische Einschränkung, bedingt durch laterale Variationen in der effektiven Schichtdicke, verstehen. Zudem ist eine raue Oberfläche gegenüber einer glatten Oberfläche, bei gleichen äußeren Abmessungen, vergrößert. Das darauf aufgedampfte Filmvolumen, berechnet zu $50 \mathrm{~nm}$ Dicke als glatte Schicht, „,verteilt" sich auf diese größere Oberfläche des Substrates und wird dadurch

\footnotetext{
${ }^{8}$ Für die dicke PdCuSi-Schicht etwa vier mal schneller als unterhalb von RT.
} 
dünner. In diesem Fall steht den internen Prozessen im Glas nicht mehr die Längenskala von $50 \mathrm{~nm}$ zur Verfügung, was das Verlustspektrum stark verändert. Die kritische Längenskala für diese Veränderung liegt also deutlich unter $50 \mathrm{~nm}$. Das Verlustspektrum (Abb. 4.22) dieser Schicht zeigt unterhalb von $590 \mathrm{~K}$ keine ansteigende Dämpfung. Man beobachtet nur eine unsystematische Schwankung der Verlustmessung mit Film um die Leermessung des Doppelpaddels. Bei $590 \mathrm{~K}$ steigt die Dämpfung mit Film deutlich an, fällt bei $625 \mathrm{~K}$ bereits wieder auf die Leermessung zurück. Aus der ebenfalls gemessenen Frequenzverschiebung ergibt sich für diesen Temperaturbereich ein Erweichen der Schicht, was auf einen $\alpha$-Prozess in diesem Temperaturbereich deutet. Eine Übersicht der mit der Erweichung bestimmten Glastemperaturen ist in Tabelle 5.1 auf Seite 113 gegeben. Das Erweichen bei einer, gegenüber einer dicken, glatten Schicht, deutlich reduzierten Temperatur geht hier mit einer niedrigeren Kristallisationstemperatur einher. Das Abfallen der Dämpfung bei $625 \mathrm{~K}$ auf die Leermessung kann nur mit einer solchen Kristallisation erklärt werden. Die niedrige Kristallisationstemperatur ist folglich ebenso eine Folge einer erhöhten Mobilität wie die reduzierte Glastemperatur. Die Kristallisation kann auch durch das raue Substrat selbst begünstigt sein, da dieses aus einer kristallisierten PdCuSi-Legierung besteht. Identifiziert man also den Ausschlag in Abbildung 4.22 als einen einsetzenden $\alpha$-Peak mit anschließender Kristallisation, so fehlt in dieser Messung jedes Indiz für einen sekundären $\beta$-Prozess. Um eine kritische Längenskala, bei der dieser Unterschied im Verlustspektrum auftritt, genauer zu studieren, wird im folgendem Abschnitt das Verhalten der dünnen glatten Filme beim Reduzieren der Schichtdicke besprochen.

\subsubsection{Analyse der dünnen Filme auf glatten Substraten}

Wie in Kapitel 5.1 .2 bereits ausgeführt, unterscheiden sich die Filme in ihrer Oberflächenrauigkeit nur sehr gering, was die Vergleichbarkeit sicherstellt. Der dünnste Film von nur $10 \mathrm{~nm}$ kann durch das mit Tantal beschichtete Substrat bereits deutlich beeinflusst sein, diese Schichtmessung wird im Abschnitt 5.5.3 separat diskutiert.

Eine Auftragung der Verlustspektren verschiedener Schichtdicken ist in Abbildung 4.27 dargestellt. In den Messungen der dünneren Schichten sind starke Dämpfungsmaxima enthalten. Um das Erkennen von Charakteristika zu erleichtern, sind mit der Begründung aus Abschnitt 5.2 die Messpunkte in dem Bereich dieser scharfen Maxima in der im Folgenden diskutierten Abbildung 5.6 herausgenommen worden. Es ergeben sich damit abschnittsweise Lücken in dem Spektrum, es wurden aber keine Messdaten gegenüber der ursprünglichen Messung verändert. 


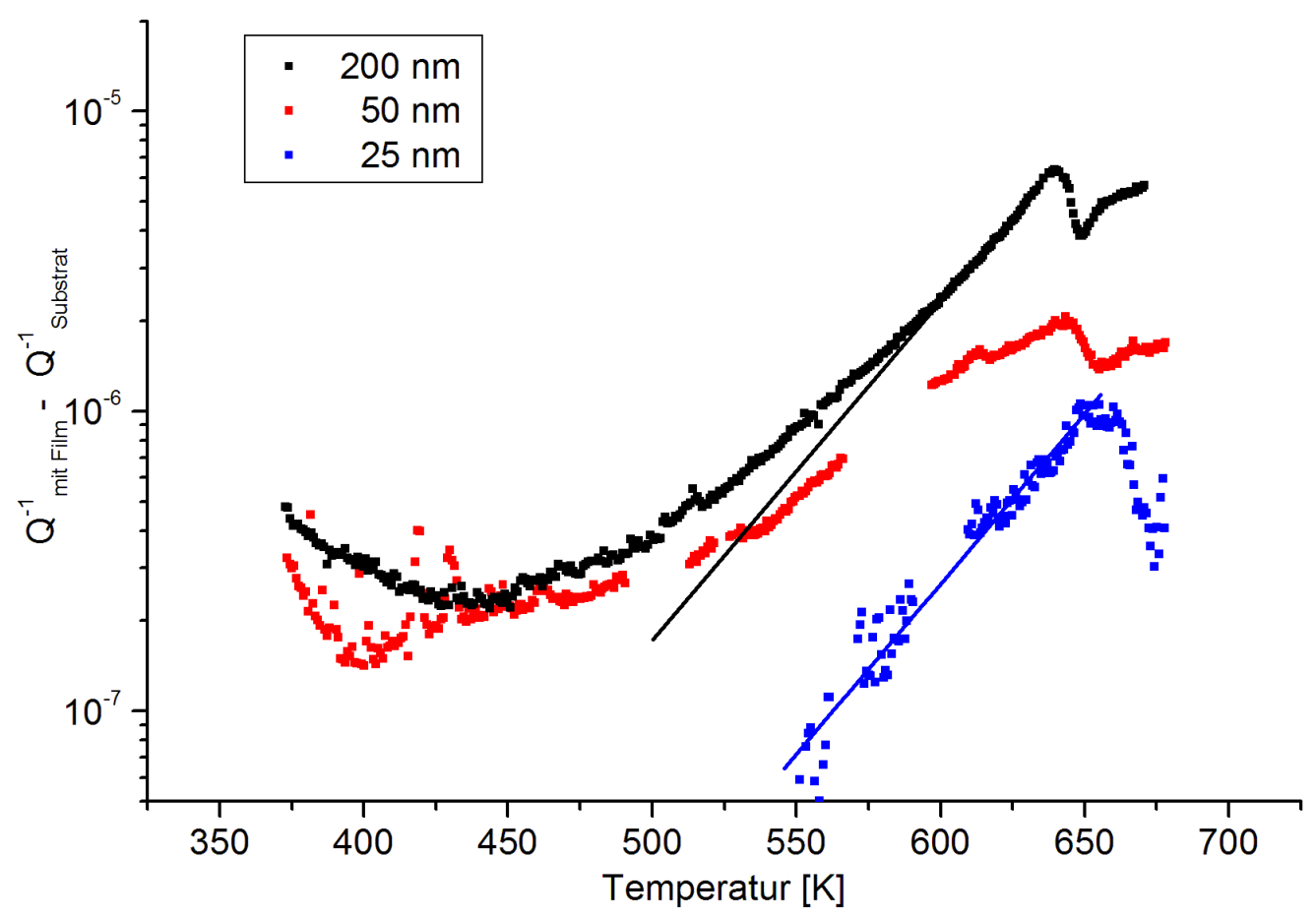

Abbildung 5.6: Übersicht der Schichtdämpfung für verschiedene Schichtdicken auf glatten Substraten. Gegenüber Abbildung 4.27 wurden die durch Dämpfungsmaxima gestörten Messpunkte herausgenommen. Die blaue Line stellt eine lineare Anpassung der Dämpfung in dem gezeigten Temperaturbereich dar. Die schwarze Linie symbolisiert diesen Verlauf vergleichend für die dickste Schicht. 
In dieser Gegenüberstellung zeigt sich der Verlauf des Verlustes bei $200 \mathrm{~nm}$ Schichtdicke sehr ähnlich zu dem Verlauf bei dem Film mit 50 nm Dicke. Im direkten Vergleich ist der Verlust insbesondere bei niedrigen Temperaturen vergleichbar, obwohl das Schichtvolumen um einen Faktor vier unterschiedlich ist. Dies kann mit der Variation in der Dämpfung bei Raumtemperatur durch den Herstellungsprozess erklärt werden. Die Variation kann beispielsweise auch in der Dämpfung und in dem Schermodul der Schichten nahe Raumtemperatur, dargestellt in den Tabellen 4.3 und 4.4, erkannt werden. Bei hoher Temperatur, bei der der $\alpha$-Prozess einsetzt, skaliert die gemessene Dämpfung deutlich mit der Schichtdicke.

Für die $25 \mathrm{~nm}$ dicke Schicht ist ein deutlich anderes Verhalten zu erkennen. Bis $550 \mathrm{~K}$ kann keine signifikante Dämpfung $\left(\Delta Q^{-1}>5 * 10^{-8}\right)$ gemessen werden, die numerische Auswertung liefert erheblich kleinere bzw. negative Werte um Null. Während der Dämpfungsverlauf der dickeren Filme insgesamt gekrümmt verläuft, tritt bei der Schichtdicke von $25 \mathrm{~nm}$ nur ein linearer Anstieg in der logarithmischen Auftragung auf. Dieser Anstieg wird mit einer Ausgleichsgerade (blaue Gerade in Abb. 5.6) gut angepasst. Der anschließende steile Abfall wird, wie bei den dickeren Schichten, mit der Kristallisation erklärt.

Die Steigung, mit der der Verlust in der dünnsten Schicht ansteigt, ist, besonders im Vergleich zu der $50 \mathrm{~nm}$ dicken Schicht, sehr groß. Durch Verschiebung der blauen Anpassungsgerade kann eine solche Steigung auch bei der $200 \mathrm{~nm}$ dicken Schicht gefunden werden. Etwa von $600 \mathrm{~K}$ bis zur Kristallisation, also in dem Temperaturbereich in dem der $\alpha$-Prozess den $\beta$-Prozess zu überlagern beginnt, entspricht der Verlauf der dünnsten Schicht dem Verlauf der dicksten Schicht bei einer Skalierung um den Faktor acht. Ordnet man diese hohe Steigung primär dem $\alpha$-Verlust zu, so erkennt man für die dickste Schicht durch die Abweichung von dieser Steigung unter $600 \mathrm{~K}$ die Separation des wings. Auffällig ist ebenfalls, dass die Steigung des Verlustes der dicksten Schicht im oberen Teil dieses, soeben identifizierten, wings näherungsweise der Steigung der mittleren Schichtdicke im oberen Temperaturbereich entspricht. Die Beeinflussung des Verlustspektrums durch Dämpfungsmaxima in diesem Temperaturbereich erschwert jedoch die Prüfung der Frage, ob es sich dabei um einen zu höherer Temperatur verschobenen wing handelt.

Auch ohne das Kriterium der Steigungen ist ein fundamentaler Unterschied im Verlustspektrum der $25 \mathrm{~nm}$ dicken Schicht allein dadurch gegeben, dass überhaupt kein messbarer mechanischer Verlust in einem großen Temperaturbereich (350-550 K) auftritt. Für die dicken Schichten ist der Verlust in diesem Temperaturbereich deutlich messbar und fällt kaum mit der Schichtdicke ab. Bei der Halbierung der Schichtdicke von $50 \mathrm{~nm}$ auf $25 \mathrm{~nm}$ fällt jedoch der Verlust unter die Messbarkeit. Die Messgenauigkeit hingegen bie- 
tet noch etwa den Faktor 10 an Empfindlichkeits-"Reserve".

Zusammenfassend zeigt dieser Vergleich das abrupte Verschwinden des wings oberhalb einer Längenskala von $25 \mathrm{~nm}$. Ob ein solcher wing bei $50 \mathrm{~nm}$ Schichtdicke ohne ausgeprägte Rauigkeit zu höherer Temperatur verschoben auftritt, kann aus den Messdaten nicht sicher verifiziert werden. Bei $50 \mathrm{~nm}$ Schichtdicke mit Einschränkungen durch Substratrauigkeiten wird das Nichtauftreten des wings ebenfalls beobachtet, wodurch sich als Abschätzung der kritischen Schichtdicke eine Längenskala von etwa $30 \mathrm{~nm}$ anbietet. Eine Modellvorstellung, die eine solche Längenskala physikalischen Prozessen zuordnet, wird in Abschnitt 5.5.5 vorgestellt. Im Folgenden werden zuvor noch weitere Messergebnisse diskutiert.

\subsubsection{Grenzfall: $10 \mathrm{~nm}$ Schichtdicke}

Die dünnste Schicht von nur $10 \mathrm{~nm}$ zeigt ein mechanisches Verhalten, welches nicht mit oben angegebener Deutung verstanden werden kann. Der gemessene Verlust, hervorgerufen durch den $10 \mathrm{~nm}$ Film (dargestellt in Abb. 4.29), ist deutlich höher als, ausgehend von $25 \mathrm{~nm}$, zu erwarten. Die Messung zeigt ab $500 \mathrm{~K}$ einen langsamen Anstieg der Dämpfung ${ }^{9}$, welcher stets oberhalb des Verlustes der $25 \mathrm{~nm}$ dicken Schicht liegt. Zudem ist bei keiner Temperatur eine Skalierbarkeit der Dämpfung im Vergleich zu den in Abbildung 5.6 gezeigten anderen Verlustmessungen gegeben. Diese Beobachtung impliziert bereits, dass hier ein anderer Verlustmechanismus vorliegt als bei den dickeren Schichten. Die Messung der Frequenzverschiebung in Abbildung 4.30 zeigt keinen Hinweis auf ein rasches Erweichen der Schicht in Form eines Glasüberganges bzw. einer $\alpha$-Relaxation. Bis etwa $550 \mathrm{~K}$ zeigt sich in dieser Messung ein kontinuierliches Erweichen, welche sich ab dieser Temperatur deutlich verlangsamt. Die Frequenzverschiebung von amorpher zu kristalliner Schicht zeigt die Änderung des Schermoduls der Schicht noch etwas deutlicher. In Abbildung 4.31 ist ebenfalls ein langsames Erweichen der Schicht beim ersten Heizen bis $550 \mathrm{~K}$ zu erkennen, anschließend nähern sich amorphe und kristalline Schicht bezüglich des Schermoduls ${ }^{10}$ langsam an. Bei $665 \mathrm{~K}$ steigt der Frequenzunterschied sehr schnell an, anschließend springt er auf etwa Null. Identifiziert man den Anstieg bei $665 \mathrm{~K}$ mit der Kristallisation, welcher auch mit dem Abfall der Dämpfung zusammenfällt, so kann der abrupte Sprung in der Frequenzdifferenz danach z. B. mit einem spontanen Aufreißen der kristallinen Schicht erklärt werden. Der langsame Anstieg im Verlust

\footnotetext{
${ }^{9}$ Der Verlust unterhalb von $500 \mathrm{~K}$ kann durch das Dämpfungsverhalten des leeren Paddels nicht genau bestimmt werden.

${ }^{10}$ Da bei diesem Vergleich der Masseanteil in Gleichung (2.27) entfällt, zeigt $\Delta \mathrm{f}$ direkt den Unterschied im Schermodul an.
} 
von $550 \mathrm{~K}$ bis zur eigentlichen Kristallisation kann nicht mit bekannten Relaxationsmoden in $\mathrm{PdCuSi}$ in diesem Temperaturbereich in Zusammenhang gebracht werden. Die Frequenzmessung in Abbildung 4.31 zeigt dabei einen kontinuierlichen Anstieg im Schermodul nahe an den Wert der kristallisierten Schicht. Es wird angenommen, dass die Wechselwirkung an der Grenzfläche zum kristallinen Tantal für das mechanische Verhalten entscheidend ist. Für amorph-kristalline Grenzflächen ist gezeigt worden, dass Atome in der amorphen Phase die Ordnung der kristallinen Phase über einige Atomabstände hinweg bereits bei niedriger Temperatur übernehmen [94]. Eine solche Grenzfläche beeinflusst, durch die Kornstruktur des Tantals (siehe Kap. 4.1.3 und 5.1.2) und die geringe Schichtdicke, bereits einen deutlichen Anteil des Volumens der $10 \mathrm{~nm}$ Schicht. Nimmt man nun an, dass sich beim Heizen ab $550 \mathrm{~K}$ eine solche geordnete Phase zu freien Oberfläche hin ausbreitet, kann der langsame Anstieg des Schermoduls erklärt werden. Bei einem solchen Prozess kann ebenfalls starke innere Reibung auftreten, womit ein solcher Prozess auch den großen Verlust in Abbildung 4.29 erklären kann. Beim Heizen nimmt die Diffusion in der Schicht weiter zu, in der Folge tritt bei $665 \mathrm{~K}$ eine (Re-)Kristallisation in die kristalline Struktur einer PdCuSi-Legierung auf. Eine andere Deutung ist ebenfalls möglich, zumal die Zusammensetzung dieser Schicht nicht so genau bekannt ist, wie für die dickeren Schichten: Falls die Zusammensetzung des Films deutlich von der Vorgabe abweicht, so kann, auch ohne eine „Kristallisationsfront“ am Substrat, lokal Kristallisation auftreten, die ebenfalls starke innere Reibung verursacht [95].

\subsubsection{Glastemperatur, Kristallisation, Schermodul und Dämpfung in Abhängigkeit der Schichtdicke}

Im Fall von organischen Glasbildnern wird zumeist der Einfluss der Schichtdicke auf die Glastemperatur untersucht [42, 40, 41]. In der vorliegenden Arbeit steht das Relaxationsspektrum metallischer Gläser bei dünnen Schichten im Vordergrund, die mechanischen Messdaten enthalten auch Information über die Glastemperatur und die Kristallisationstemperatur. Die sehr genauen Messungen nahe Raumtemperatur liefern zudem Schermodul und Dämpfung in Abhängigkeit der Schichtdicke. Alle diese Abhängigkeiten sollen in diesem Abschnitt diskutiert werden.

\section{Schermodul:}

Eine tabellarische Auflistung der bestimmten Schermodule der verschiedenen dünnen Schichten nach der Herstellung in Tabelle 4.3 zeigt keine signifikante Variation des Schermoduls mit der Schichtdicke. Bei dünneren Filmen nimmt methodisch bedingt der Messfehler stark zu, hier wird die Bestimmung un- 
sicherer. Der rein elastische Schermodul ohne angeregte interne Prozesse ist durch die Wechselwirkung direkt benachbarter Atome bestimmt. Daher wird nahe Raumtemperatur in dem hier studierten Schichtdickenbereich auch keine Veränderung des Moduls erwartet.

\section{Dämpfung:}

Die zu erwartende Dämpfung $\Delta Q^{-1}$ der Schichten ist, nach Gleichung (2.30) und (2.32), bei gleichem Verlustmodul $G^{\prime \prime}$ der Filme direkt proportional zur Schichtdicke. Die Übersichtstabelle 4.4 zeigt die tatsächlich gemessenen Dämpfungen für verschiedene Schichtdicken. Wie bereits in Kapitel 4.2.6 ausgeführt, ist die Abhängigkeit von der Schichtdicke nahe Raumtemperatur nicht klar zu erkennen. Obwohl für dünnere Schichten immer kleinere Dämpfungen gemessen werden, kann aus den scheinbar stark streuenden Messwerten keine direkte Abhängigkeit entnommen werden. Auch wenn Messungenauigkeiten dabei eine Rolle spielen können, insbesondere durch Dämpfungsmaxima nahe Raumtemperatur, so sind die gemessenen Variationen damit allein nicht zu erklären. Die Dämpfungsunterschiede zeigen deutlicher die durch die Herstellung bedingten Unterschiede der Schichten, als eine SchichtdickenAbhängigkeit. Mit fortschreitender Relaxation der Schichten ist zu erwarten, dass diese Unterschiede, die durch verschiedene Startpositionen in der PEL beschrieben werden können, sich ausgleichen.

\section{Glastemperatur und Kristallisationstemperatur:}

Die Glastemperatur ist (siehe Kap. 1.2.1) keine eindeutig festgelegte Größe, sie wird hier bei einer Spektroskopiefrequenz von etwa $5,4 \mathrm{kHz}$ mit einer Heizrate von $1 \mathrm{~K} / \mathrm{min}$ folgendermaßen bestimmt: In der Auftragung der Frequenzverschiebung durch den Film gegen die Temperatur kann in einigen Spektren ein schnelles Abfallen des Schermoduls erkannt werden. Der Beginn dieses Abfalls, bestimmt durch eine Tangentenkonstruktion, wird als Glastemperatur festgelegt.

Die Kristallisation des Filmes führt bei allen Verlustmessungen bei einer bestimmten Temperatur zu einem Abfall der Dämpfung $\Delta Q^{-1}$. Diese Temperatur wird als Kristallisationstemperatur verwendet.

Die folgende Tabelle 5.1 zeigt diese so bestimmten Temperaturen vergleichend. Nicht für alle Schichten konnte eine Glastemperatur bestimmt werden ${ }^{11}$. Der Fehler bei der Bestimmung dieser Temperaturen wird mit $5 \mathrm{~K}$ geschätzt.

Eine graphische Auftragung dieser Messwerte zeigt die Abbildung 5.7.

Im Rahmen des Fehlers erkennt man bezüglich der Glasübergangstemperatur keine Schichtdicken-Abhängigkeit. Nur die Schicht auf dem rauen Substrat

\footnotetext{
${ }^{11}$ Bei DPO_DB_08 wird diese Bestimmung durch den Einfluss der Dämpfungsmaxima auf die Frequenzlage verhindert, bei DPO_DB_13 wird kein solcher Prozess beobachtet.
} 


\begin{tabular}{|c|c|c|c|}
\hline Probe & $\begin{array}{c}\text { nom. Schichtdicke } \\
{[\mathrm{nm}]}\end{array}$ & $\begin{array}{c}T_{g} \\
{[\mathrm{~K}]}\end{array}$ & $\begin{array}{c}T_{x} \\
{[\mathrm{~K}]}\end{array}$ \\
\hline DPO_DB_05 & 200 & 625 & 639 \\
\hline DPO_DB_07 & 50 & 632 & 646 \\
\hline DPO_DB_05a & $50\left(^{*}\right)$ & 597 & 613 \\
\hline DPO_DB_08 & 25 & n. b. & 656 \\
\hline DPO_DB_13 & 10 & n. b. & 668 \\
\hline
\end{tabular}

Tabelle 5.1: Darstellung der Glasübergangs $\left(T_{g}\right)$ - und Kristallisationstemperaturen $\left(T_{x}\right)$ für verschiedene Schichtdicken. Die Angabe $\left(^{*}\right)$ bei der Schichtdicke markiert die Messung auf dem rauen Substrat.

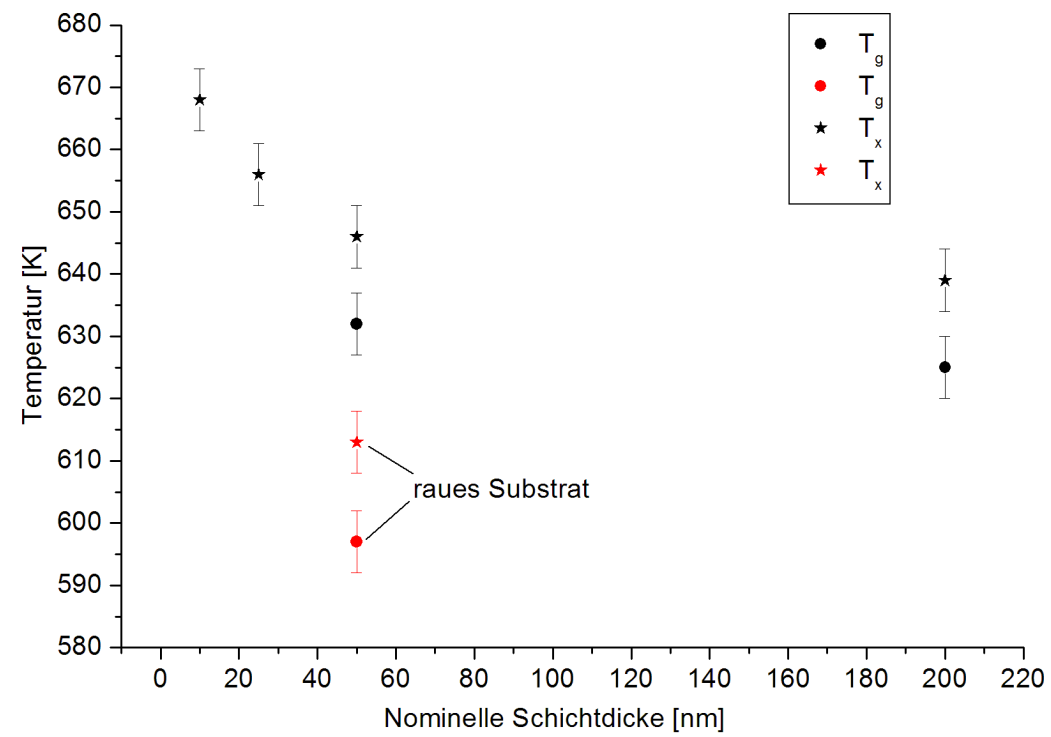

Abbildung 5.7: Abhängigkeit von Glastemperatur $\left(T_{g}\right)$ und Kristallisationstemperatur $\left(T_{x}\right)$ von der Schichtdicke nach Tabelle 5.1. 
von nominell $50 \mathrm{~nm}$ zeigt eine deutlich abgesenkte Glastemperatur. In Abschnitt 5.5.1 wurde bereits eine effektiv verringerte Schichtdicke durch das raue Substrat erläutert. Adaptiert der Film die Form des rauen Substrates, so weist auch dieser eine stark vergrößerte Oberfläche auf. Freie Oberfächen führen i. A. zu einer lokal erniedrigten Glastemperatur [43] bzw. zu einer Oberfläche mit flüssigkeits-artiger Dynamik [96]. Mit der gegenüber der glatten $50 \mathrm{~nm}$ Schicht stark vergrößerten Oberfläche kann die, nur auf dem rauen Substrat beobachtete, reduzierte Glastemperatur erklärt werden. Die reduzierte Glastemperatur steht nicht im Widerspruch zu den Messungen an den glatten Filmen, da die effektive Schichtdicke deutlich unter $50 \mathrm{~nm}$ liegt, und in diesem Bereich keine Glastemperatur bei den glatten Filmen bestimmt werden kann.

Betrachtet man nur die Filme auf glatten Substraten, so zeigt die Kristallisationstemperatur eine klare Tendenz. Mit Abnahme der Schichtdicke steigt die Kristallisationstemperatur bei dünnen Schichten deutlich an. Eine solche Tendenz wurde auch in der Arbeit von C. Weiß an dünnen Multilagen von $\mathrm{ZrAlCu} / \mathrm{Ta}$ beobachtet [97]. Sowohl in [97] als auch in dieser Arbeit können Wechselwirkungen zum Substrat als Ursache geltend gemacht werden. Der amorphe Film passt sich an jedes Substrat ohne Fehlanpassung an, bei der Kristallisation ändert sich das Volumen des Filmes, die dazu nötige Verzerrungsenergie konkurriert mit dem Enegiegewinn der Kristallisation. Da dieser Beitrag die Kristallisation behindert und mit sinkender Schichtdicke an Bedeutung gewinnt, ist die steigende Kristallisationstemperatur als eine Folge dieser Barriere zu verstehen. Der Film auf dem rauen Substrat (in Abb. 5.7 in rot dargestellt) weist im Gegensatz zu den glatten Filmen eine deutlich tiefere Kristallisationstemperatur auf. Wie in Abschnitt 5.5.1 diskutiert, kann die niedrige Kristallisationstempertaur der rau aufgedampften Schicht sowohl in Folge der erhöhten Oberfläche, als auch durch erleichterte Kristallisation ausgehend von dem Substrat aus kristallinem PdCuSi bedingt sein. Ein Volumensprung an der Grenzfläche bei der Kristallisation ist für die amorphe, auf kristallisiertem PdCuSi aufgedampfte, Schicht nicht zu erwarten, da die Gitterstuktur der kristallinen PdCuSi-Legierung an der Grenzfläche bereits bei der Herstellung adaptiert werden kann. Damit ist auch zu verstehen, weshalb die effektiv geringe Schichtdicke in diesem Fall nicht die Kristallisationstemperatur erhöht.

\subsubsection{Modellbildung}

Die Verschiebungen von Glastemperatur und Kristallisationstemperatur bei Reduzierung der Schichtdicke konnten bisher ausschließlich mit den Einflüssen von Oberfläche und Grenzfläche erklärt werden. Die gemessenen Verlust- 
spektren sind in Abschnitt 5.5.2 bereits analysiert und verglichen worden, damit ist noch keine physikalische Deutung gegeben. An dies Stelle soll nun ein Modellbild vorgeschlagen werden, mit dem das "Verschwinden" des wings erklärt werden kann. Unabhängig davon ob die Schicht auf einem rauen oder glatten Substrat hergestellt ist, unterhalb einer kritischen Schichtdicke wird kein wing im Verlustspektrum beobachtet. Zum Verständnis dieses NichtAuftretens gibt es zwei verschiedenen Hypothesen, die zu verschieden Erklärungsansätzen führen:

1. Die eine Hypothese ist, dass das Verlustspektrum gegenüber den dicken Schichten generell unverändert bleibt, mit dem einzigen Unterschied, dass der langsame $\beta$-Prozess nicht mehr auftritt.

2. Die andere Hypothese lautet, dass der Übergang von einer dreidimensionalen zu einer zunehmend zweidimensionalen Geometrie des Films die auftretenden kollektiven Prozesse soweit beeinflusst, dass eine Trennung von $\alpha$ und $\beta$-Prozess nicht mehr auftritt.

Ausgehend von erster Hypothese stellt sich die Frage, warum der $\beta$-Prozess bei einer kritischen Längenskala von etwa $30 \mathrm{~nm}$ nicht mehr als Verlustprozess auftritt. In dem Kapitel 1.4 wurden bereits einige bekannte Längenskalen in Gläsern vorgestellt. Von diesen bekannten Längenskalen gibt es bisher keine vergleichbar große Längenskala, wie die hier als kritisch auftretende. Die Kopplung zwischen einzelnen Atomen reicht in amorphen Systemen typischerweise über einige Bindungslängen, wie in der Abbildung 1.7 für die radiale Strukturfunktion gezeigt. Der Vorschlag für eine so große Längenskala beinhaltet daher elastische Felder. Elastische Felder sind ein elementarer Bestandteil bei der Modellierung dynamischer Heterogenitäten wie Schertransformationszonen (STZ). Ein plastischer Kern einer STZ ist von einem elastisch verspannten Bereich umgeben, in dem die Deformationsenergie der STZ gespeichert ist [39]. Ein solches Spannungsfeld wird als Eshelby-Spannungsfeld bezeichnet [98]. Der $\beta$-Prozess wird mikroskopisch mit string-Prozessen in Verbindung gebracht. Es ist nun in direkter Analogie zu den Schertransformationszonen plausibel, dass auch solche ketten-artigen Bewegungen mit einem elastischen Spannungsfeld verknüpft sind. Die genaue Größe, bzw. die Zahl der beteiligten Atome, von solchen Ketten ist nicht gut bekannt. In Simulationen sind string-artige Verschiebungen von Atomen über einige Nanometer zu bestimmen [10], und Abschätzungen von H. R. Schober [23] ergeben eine Kopplung von 20-100 Atomen bei tiefen Temperaturen. Nimmt man nun zunächst eine Ausdehnung der strings von etwa $10 \mathrm{~nm}$ an, so kann mit einem drei mal so großen Spannungsfeld eine vergleichbare Größenskala, wie die hier beobachtete kritische, konstruiert werden. 


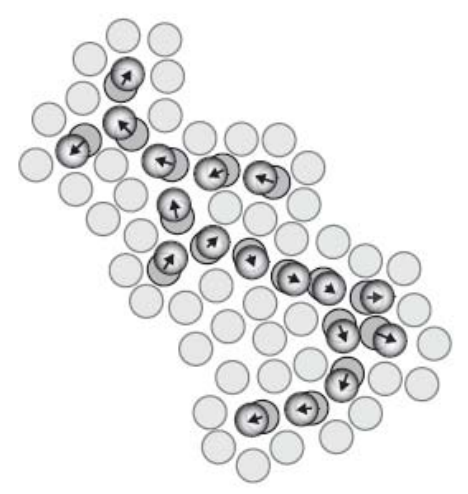

Abbildung 5.8: Schematische Darstellung eines dreidimensionalen $\beta$ Prozesses in Form eines Quadrupoles. Die Abbildung ist aus [99] entnommen, nach [23].

Ein schematisches Bild für einen (dreidimensionalen) $\beta$-Prozess zeigt die Abbildung 5.8. Dabei sind die atomaren Verschiebungen in Form eines Quadrupoles angeordnet. Eine solche Anordnung kann aufgrund des schnell abfallenden elastischen Spannungsfeldes energetisch günstig sein. Im einfacheren Fall ist auch eine Dipol-artige Bewegung möglich, die von einem ausgedehnten Spannungsfeld umgeben ist. Ein schematisches Bild für eine Dipol-artige Bewegungsmode mit einem elastischen Feld ist in Abbildung 5.9 gezeichnet. Die Ausdehnung eines solchen Spannungsfeldes kann mit einer geringen Schichtdicke konkurrieren, wodurch es zu einer Beeinflussung des $\beta$-Prozesses kommt.

In kristallinen Materialien stellen Versetzungen linienhafte Gitterfehler dar. Versetzungen sind ebenfalls von elastischen Spannungsfeldern umgeben. Diese ausgedehnten Spannungsfelder führen im kristallinen Gitter zu Wechselwirkungen von Versetzungen untereinander, aber auch zu Wechselwirkungen mit freien Oberflächen. Nahe von Oberflächen treten so genannte Bildkräfte auf, die zu einer Anziehung an die Oberfläche und auch zu Annihilation der Defekte führen [75]. Die Wechselwirkungsenergie pro Längeneinheit $E_{W W}$ einer Schraubenversetzung mit der virtuellen, gespiegelten Versetzung ist gegeben durch [75]:

$$
E_{W W}(r)=\frac{G b^{2}}{4 \pi} \ln \frac{r}{r_{0}}
$$

Dabei bezeichnet $G$ den Schermodul, $b$ den Betrag des Burgersvektors der Schraubenversetzung, $r$ den Abstand von der Oberfläche und $r_{0}$ den Kernradius der Versetzung. Die wechselwirkende Kraft, gegeben durch die radiale Ableitung dieser Funktion, fällt damit proportional zu $1 / r$ bei Entfernung von der Oberfläche ab, also sehr rasch. 


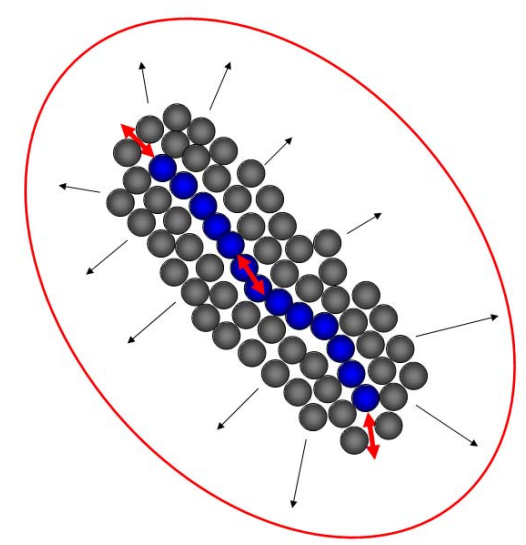

Abbildung 5.9: Schematische Darstellung eines $\beta$-Prozesses in Form eines Dipols mit angedeutetem Spannungsfeld.

Mit dieser Vorstellung ist das Nichtauftreten eines wings dadurch zu erklären, dass string-Prozesse, als die mikroskopische Ursache des $\beta$-Prozesses, sehr schnell an der freien Oberfläche annihilieren und damit nicht weiter zur Dämpfung beitragen können. Oberhalb der kritischen Schichtdicke ist die zur Oberfläche ziehende Kraft zu gering, um diese Prozesse zu vernichten und die Kettenanregungen stellen einen mechanischen Verlustprozess dar, der den wing im Verlustspektrum hervorruft.

Der $\alpha$-Prozess wird mikroskopisch durch Schertransformationszonen beschrieben. Bei einem Durchmesser von etwa 1,5 nm [10] kann, auch mit einem drei mal so ausgedehnten elastischen Feld, keine ausgeprägte Wechselwirkung mit einer kritischen Schichtdicke von $30 \mathrm{~nm}$ erwartet werden. Bei dem Vergleich der Verlustspektren bis zu der dünnsten Schichtdicke von $25 \mathrm{~nm}$ wird kein Einfluss auf den Verlust bei entsprechend hohen Temperaturen beobachtet. Der Verlust, der in Abbildung 5.6 dem Bereich des $\alpha$-Prozesses zugeordnet wird (ab $600 \mathrm{~K})$, skaliert direkt mit Schichtdicke und Temperatur.

Die Messung der $10 \mathrm{~nm}$ dicken Schicht zeigt ein von den dickeren Schichten deutlich abweichendes Verhalten. Die Messung der Frequenzverschiebung zeigt insbesondere keinen Hinweis auf eine schnelle Erweichung. Die Schicht zeigt damit auch keinen $\alpha$-Prozess. Die Bedeutung der Wechselwirkung zum Substrat ist bereits in Abschnitt 5.5.3 diskutiert. Mit dem soeben vorgestellten Modell kann das Nicht-Auftreten eines $\alpha$-Prozesses auch durch eine Wechselwirkung des $\alpha$-Prozesses mit der Schichtdicke erklärt werden. Da ein gewisser Anteil der $10 \mathrm{~nm}$ Schicht durch das Substrat bereits ,festgehalten“ wird, ist es möglich, dass der verbleibende „mobile“ Anteil der Schicht bereits zu schmal für den sphärischen $\alpha$-Prozess ist. 


\section{Zwischenfazit:}

Eine Diskussion der Messungen über das rein geometrische Argument der Größe der Prozesse und ihrer Wechselwirkungen mit der Schichtdicke bietet die Basis, experimentell eine Abschätzung der relevanten Längenskalen zu gewinnen. Damit wird es möglich, die Längenskala von Heterogenitäten experimentell zu vermessen, im Gegensatz zu den bisher vorliegenden Erkenntnissen aus Simulationen oder Modellanpassungen an indirekte mechanische Messungen (z. B. über das Aktivierungsvolumen [100]).

Nachteilig an einer solchen Argumentation ist die stark vereinfachende erste Hypothese, dass die charakteristischen Verlustprozesse selbst nicht von der Dimensionalität der Probe abhängen. Auch ein möglicher Zusammenhang von $\alpha$ - und $\beta$-Prozess wird mit dieser Argumentation außer Acht gelassen. Um solche Fragen ebenfalls mit den hier gezeigten Messungen zu diskutieren, wird eine zweite Interpretationsmöglichkeit besprochen.

Die zweite Hypothese, die das Nicht-Auftreten des wings unter $30 \mathrm{~nm}$ erklären kann, besagt, dass eine Trennung von $\alpha$ - und $\beta$-Prozess bei zweidimensionalen Strukturen nicht mehr auftritt. Der Vorgang, dass beide Prozesse nicht mehr getrennt auftreten, wird als merging bezeichnet. Die merging-Temperatur, oberhalb der $\alpha$ - und $\beta$-Prozess gleichzeitig auftreten bzw. nicht mehr voneinander unterschieden werden können, wurde für $\mathrm{PdCuSi} \mathrm{zu}$ $720 \pm 110 \mathrm{~K}$ bestimmt [31]. Diese Temperaturangabe gilt aber nur bei massiven Proben. Für geometrisch eingeschränkte Gläser ist es eine plausible Annahme, dass sich die Form der elementaren Moden deutlich gegenüber denen für massive Gläser ändert. Mit dieser Veränderung verschiebt sich auch die Temperaturabhängigkeit der Moden und damit die merging-Temperatur. Die Tatsache, dass bei dünnen Schichten unterhalb der kritischen Schichtdicke nur ein Verlustprozess beobachtet wird, kann prinzipiell auch als eine Folge eines geometrisch bedingten merging-Prozesses gedeutet werden. Mit dieser Interpretation ist der Anstieg im Verlust der $25 \mathrm{~nm}$ dicken Schicht in Abbildung 5.6 als $\alpha, \beta$-Prozess und nicht als reiner $\alpha$-Prozess zu identifizieren.

Im Folgenden sollen mögliche Ursachen eines solchen geometrischen mergings besprochen werden. Das Bild für einen merging-Prozess als Funktion von Temperatur und Frequenz bzw. Zeitkonstante ist in Abbildung 5.10 aufgezeigt. Die unterschiedliche Temperaturabhängigkeit von $\alpha$ - und $\beta$-Prozess führen bei hoher Temperatur bzw. hoher Frequenz zu einen gemeinsamen Verlauf der Prozesse.

Damit dieser Vorgang nur durch eine Reduzierung der Schichtdicke abläuft, ist eine Beeinflussung der charakteristischen Prozesse mit der Schichtdicke 


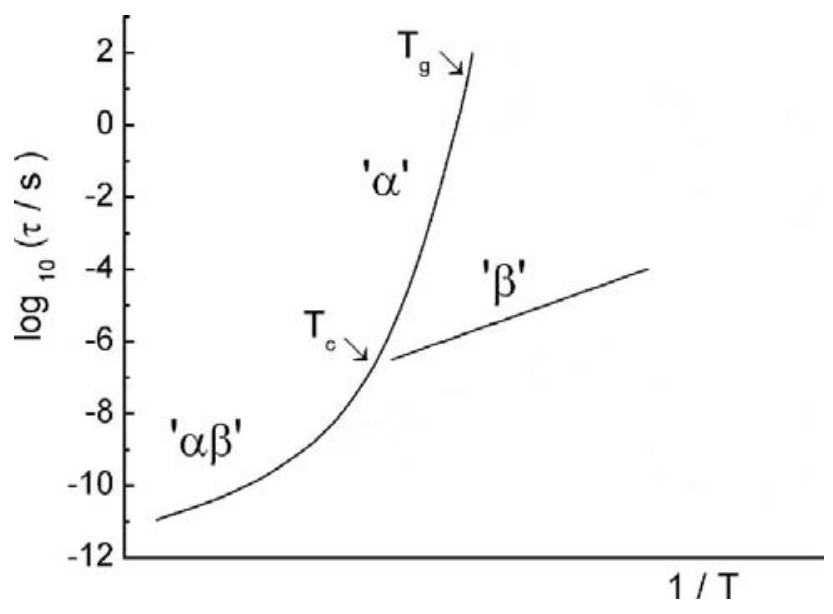

Abbildung 5.10: Der merging-Prozess als Funktion der Temperatur und Frequenz in schematischer Darstellung, aus [31].

nötig. In einem einfachen Szenario verschiebt der $\alpha$-Prozess in Abbildung 5.10 zu tieferen Temperaturen und es reduziert sich die merging-Temperatur. Eine Absenkung des $\alpha$-Prozesses ist in Abbildung 5.6 und auch bei den Glastemperaturen in Tabelle 5.1 für die glatten Filme nicht zu erkennen. Eine reduzierte Glastemperatur konnte nur bei der $50 \mathrm{~nm}$ Schicht auf dem rauen Substrat beobachtet werden. In diesem Fall wird die Absenkung durch Oberflächeneffekte und nicht durch Längenskaleneffekte erklärt. Eine solche Verschiebung des $\alpha$-Prozesses mit der Schichtdicke erscheint vor dem Hintergrund der hier vorliegenden Messungen nicht die Ursache eines möglichen geometrischen mergings zu sein. Damit bleibt eine Verschiebung des $\beta$-Prozesses in Richtung des $\alpha$-Prozesses. Es gibt in Abbildung 5.6 ein schwaches Indiz ${ }^{12}$ für diese These. Der Verlust des $50 \mathrm{~nm}$ dicken Film verläuft bei hoher Temperatur deutlich flacher als bei der dickeren und der dünneren Schicht. Dieses kann als eine Verschiebung des $\beta$-Verlustes zu höherer Temperatur gegenüber der dickeren Schicht gedeutet werden. In diesem Bild ist die Verschiebung zu dem $\alpha$-Prozess bei der $25 \mathrm{~nm}$ Schicht abgeschlossen und es tritt nur der gemeinsame $\alpha, \beta$-Prozess auf.

Die beiden hier vorgestellen Modelle stehen scheinbar unvereinbar nebeneinander. Eine Entscheidung, welche der beiden Hypothesen zutrifft, kann aus dem Verlustmessungen allein nicht getroffen werden. Es bedarf erweiterter theoretischer Modelle, die auch die Zusammenhänge von $\alpha$ und $\beta$-Prozess beschreiben können, um das Verständnis zu charakteristischen Längenskalen

\footnotetext{
${ }^{12}$ Die Beeinflussung durch Dämpfungsmaxima in dem für diese Frage relevanten Temperaturbereich von 550 bis $650 \mathrm{~K}$ führt zu einer eingeschränkten Interpretierbarkeit.
} 
zu vertiefen. Es bleibt als gesicherte Erkenntnis das Auftreten einer kritischen Längenskala von etwa $30 \mathrm{~nm}$, die im Folgenden mit bekannten Längenskalen in amorphen und kristallinen Materialien verglichen wird.

\subsubsection{Vergleich zu anderen Längenskaleneffekten amor- pher und kristalliner Materialien}

Die Suche nach Längenskalen-Effekten in Materialien ist ein aktuelles Gebiet der Festkörper- und Materialphysik. Für z. B. organische Glasbildner wird eine Reduktion der Glastemperatur, je nach Polymer, unterhalb von $100 \mathrm{~nm}$ bzw. unterhalb von $40 \mathrm{~nm}$ beobachtet [43, 101]. Für metallische Gläser wird eine erheblich kleinere Längenskala hierfür erwartet. In einer Simulationsstudie von H. Teichler zeigen sich erhöhte Diffusionskonstanten an freien Oberflächen von NiZr nahe der Glastemperatur nur im Bereich von etwa einem Nanometer [96]. Die Vorstellung, dass STZs mit einem Durchmesser von etwa 1,5 nm die mikroskopische Ursache der Plastizität sind [10], deckt sich mit der Beobachtung, dass in dem in dieser Arbeit studiertem Schichtdickenbereich kein direkter Einfluss der Schichtdicke auf die Glastemperatur oder einen einsetzenden $\alpha$-Prozess zu beobachten ist. Der Längenskalenbereich von etwa $30 \mathrm{~nm}$, der hier als kritisch auftritt, ist nach aktuellem Kenntnisstand bisher nicht als charakteristisch in metallischen Gläsern aufgetreten. Im vorherigen Kapitel wurde vorgeschlagen, dass diese Längenskala die typische Längenskala für $\beta$-Prozesse mit entsprechendem elastischen Spannungsfeld darstellt. Die Vorstellung, dass unterhalb einer kritischen Systemgröße dieser $\beta$-Prozess nicht auftritt, ist vergleichbar zu einer aktuellen Studie zur plastischen Deformation von amorphem $\mathrm{Pd}_{77} \mathrm{Si}_{23}$ [102]: Verformungsexperimente durch uniaxiale Kompression von zylindrischen Säulen zeigen einen qualitativen Größeneffekt der Deformation. Bei Säulen mit entsprechend großem Durchmesser erfolgt die Deformation heterogen, bei einem Durchmesser von $400 \mathrm{~nm}$ und weniger wird homogene Verformung beobachtet. Die heterogene Verformung in Gläsern erfolgt allgemein über sogenannte Scherbänder. In [102] wird abgeschätzt, dass die Längenskala von $400 \mathrm{~nm}$ etwa die kritische Längenskala darstellt, um durch Verformungsenergie ein Scherband zu erzeugen. In vergleichbarer Weise kann es, für die dünnen Schichten energetisch ungünstig sein, einen (vom $\alpha$-Prozess separierten) $\beta$-Prozess unterhalb der kritischen Dicke von $30 \mathrm{~nm}$ zu erzeugen.

Die $\beta$-Prozesse in amorphen Materialen können mit Versetzungen in kristallinen Materialen verglichen werden. Versetzungen haben einen großen Einfluss auf die Mechanismen der Deformation kristalliner Stoffe [75]. Verformungsexperimente an kleinskaligen Säulen aus Gold zeigen ebenfalls einen starken 
Effekt der Probengröße [103]. Sowohl bei Reduzierung der Probengröße im Bereich von 0,2-8 $\mu \mathrm{m}$, als auch bei fortschreitender Deformation, wird eine Härtung der Proben gemessen. In [103] wird daher argumentiert, dass die die Zahl der Versetzungen bei der fortschreitenden Deformation durch Oberflächenannihilation abnimmt, bzw. eine kleinere Säule bereits weniger Versetzungen aufweist. Im sub-Mikrometer-Bereich treten solche Versetzungen nur noch vereinzelt in Kristalliten auf [104], was ebenfalls zu einer starken Größenabhängigkeit der Verformungsmechanismen führt.

Diese Vergleiche zu anderen Längenskaleneffekten zeigen den universellen Charakter kritischer Längen in mechanischen Experimenten. Solche Experimente sind ein praktikabler und eleganter Weg mikroskopische Prozesse und ihre intrinsischen Längenskalen zu studieren. 


\section{Kapitel 6}

\section{Zusammenfassung}

In dieser Arbeit wurde für metallische Gläser der Einfluss der Einschränkung einer räumlichen Dimension auf das Relaxationsspektrum untersucht.

Dabei wurde die Doppelpaddel-Oszillator (DPO) Methode [12] verwendet. Mit der Nutzung der besonders geformten, in Resonanz betriebenen, Oszillatoren können mechanische Eigenschaften dünner Schichten als Funktion der Temperatur gemessen werden. Die genaue Vermessung von Resonanzfrequenz und Oszillator-Güte vor und nach dem Aufbringen des zu untersuchenden Filmes ermöglicht sowohl den Realteil als auch den Imaginärteil (Verlustanteil) des Schermoduls zu bestimmen. Studiert wurden Filme des fragilen metallischen Glases PdCuSi mit verschiedenen Dicken. Die Schichten wurden mit Elektronenstrahlverdampfern im Ultrahochvakuum auf die Oszillatoren und gleichzeitig auf jeweils ein Referenzsubstrat aufgedampft. Die Referenzsubstrate erlauben eine einfache Charakterisierung der hergestellten Schichten in Bezug auf Schichtdicke, Rauigkeit, Zusammensetzung und Amorphizität. Mit den Analysen der Referenzsubstrate konnte sichergestellt werden, dass bei der Variation der Schichtdicke die übrigen Parameter konstant blieben. Es wurde ein Film mit einer Dicke von $200 \mathrm{~nm}$ als Referenz für massive PdCuSi-Filme vermessen. Aus vorherigen Messungen an massiven Bändern des fragilen Glasbildners PdCuSi [7] und des starken Glases $\mathrm{ZrAlCu}$ [6] ist bekannt, dass der sekundäre Verlustprozess in fragilen Gläsern stärker ausgeprägt ist. Dem gegenüber zeigt der dicke Film aus $\mathrm{PdCuSi}$ in dieser Arbeit einen noch stärker ausgeprägten sekundären Verlustprozess in Form eines wings, der sich von $440 \mathrm{~K}$ bis nahe an die Kristallisationstemperatur von $640 \mathrm{~K}$ erstreckt. Die Trennung eines primären, viskos bedingten $\alpha$-Prozesses von dem sekundären $\beta$-Prozess ist im Verlustspektrum nur wenig ausgeprägt. Dies kann mit der hohen Spektroskopiefrequenz von $5,4 \mathrm{kHz}$ und der damit bedingten Nähe zu einem merging-Punkt erklärt werden [4]. Der schnelle Abfall des Schermoduls bei der Glastemperatur, deutlich vor der Kristallisation, 
zeigt, dass ein Bereich der unterkühlen Schmelze vermessen werden konnte. Bezüglich der Alterungsprozesse in frisch hergestellten Gläsern konnten ebenfalls weitere Erkenntnisse gewonnen werden. Der mechanische Verlust ist in den dicken Schichten bereits bei Raumtemperatur (RT) gut zu bestimmen. Das Einsetzen von Alterungsprozessen beim ersten Aufheizen ist direkt zu verfolgen. Es zeigt sich eine Abnahme des Verlustes direkt oberhalb von RT bis zu der Temperatur, bei der der wing einsetzt. Gleichzeitig wird ein Ansteigen des Schermoduls bis zu dieser Temperatur und mit dem wing ein langsames Erweichen gemessen. Damit ist gezeigt, dass der Vorgang der $\beta$-Alterung sich gleichermaßen auf Realteil und Imaginärteil des Schermoduls auswirkt. Sowohl das anfängliche Ansteigen des Schermoduls, als auch die langsame Abnahme während des wings, können mit dem Bild eines Potentielle-EnergieLandschaft-Modells [83] erklärt werden.

Mit dem neu konstruiertem Aufbau kann auch der Temperaturbereich von $200 \mathrm{~K}$ - RT spektroskopisch vermessen werden. In diesem Temperaturbereich zeigt sich ein linearer Abfall der Dämpfung mit sinkender Temperatur. Gleichzeitig zeigt das Schermodul der Schicht einen linearen Anstieg. In diesem Temperaturbereich wird kein weiter Verlustprozess beobachtet, der Verlauf ist konsistent mit einem Ausfrieren des slow $\beta$-Prozesses.

Wichtigster Teil dieser Arbeit ist die Analyse dünner Schichten. Durch methodische Optimierungen ist es mit dem verwendeten Messaufbau möglich, amorphe Filme von nur $10 \mathrm{~nm}$ zu vermessen. Das rechnerische Limit liegt bei etwa $2 \mathrm{~nm}$. Die Verlustspektren dünner Filme $(\mathrm{d}<50 \mathrm{~nm})$ weisen einen deutlichen qualitativen Unterschied zu denen dickerer Schichten auf. Es gibt bei den dünnen Schichten keinen erkennbaren $\beta$-Verlust mehr. Unabhängig davon, ob die Filme auf glatte oder raue Substrate abgeschieden werden, ist unterhalb einer kritischen Längenskala kein wing mehr messbar. Diese kritische Längenskala kann mit $30 \mathrm{~nm}$ abgeschätzt werden. Der mechanische Verlust der Schichten ab etwa $600 \mathrm{~K}$, also in dem Temperaturbereich, in dem der $\alpha$-Verlust dominiert, zeigt keine Abhängigkeit von der Schichtdicke im Bereich von 25 bis $200 \mathrm{~nm}$.

Die weitere Reduzierung auf $10 \mathrm{~nm}$ Dicke zeigt ein anderes Verlustspektrum. Man beobachtet überproportional ausgeprägte Dämpfung über einen weiten Temperaturbereich, welche nicht mit den bekannten Verlustprozessen vergleichbar ist. In diesem Fall sind starke Wechselwirkungen mit dem Substrat die wahrscheinlichste Ursache für das mechanische Verhalten.

Um die kritische Längenskala von $30 \mathrm{~nm}$ mikroskopisch zu deuten, werden kollektive atomare Anregungen in Form von Ketten-Bewegungen der Atome diskutiert. In Molekulardynamik-Simulationen sind sogenannte stringProzesse als mikroskopische Ursache des $\beta$-Prozesses identifiziert worden [9]. Die Verzerrung der umgebenden Matrix durch diese Bewegung in der festen 
Struktur führt zu weitreichenden elastischen Spannungsfeldern. Hierbei besteht eine Analogie zu Eshelby-Spannungsfeldern [98] von STZs (shear transformation zones) und Verzerrungsfelder um Versetzungen in kristallinen Materialien [75]. Die Ausdehnung des Spannungsfelds eines $\beta$-strings kann mit der beobachteten kritischen Längenskala der Schichtdicke konkurrieren. Es kommt damit, wie bei sehr dünnen kristallinen Filmen, zu einer Annihilierung der strings an der Oberfläche oder Grenzfläche in Folge von Bildkräften. Eine andere Art der Interpretation ist die Deutung des Nicht-Auftretens des $\beta$-Prozesses mit einem geometrisch bedingten merging-Prozess. Dabei verschiebt sich der $\beta$-Prozess in der eingeschränkten Geometrie so, dass nur ein gemeinsamer $\alpha, \beta$-Prozess bei hoher Temperatur beobachtet wird.

Für immer dünnere Schichten ist in jedem Fall eine Reduzierung des $\beta$ Verlustes zu erwarten, da in einer wirklich zweidimensionalen Geometrie weniger Moden für die string-Prozesse existieren, als in der dreidimensionalen. K. Samwer und W. L. Johnson berechnen aus dem Spannungs-Dehnungstensor 32 mögliche $\beta$-Moden in dreidimensionalen Gläsern, während in zweidimensionalen Gläsern nur 4 verschiedene Moden existieren [105].

Auch die These, dass der Effekt der Dimensionalität den stark reduzierten $\beta$ Verlust in dieser Arbeit verursacht, führt zu dem Schluss, dass die relevante Längenskala des $\beta$-Prozesses so groß ist, dass ein etwa $30 \mathrm{~nm}$ dicker Film hier bereits zweidimensional erscheint. 


\section{Anhang A}

\section{Anhang}

\section{A.1 Weitere Röntgenmessungen}

Ergänzend werden noch exemplarisch einige weitere Röntgenmessungen gezeigt, die im Ergebnissteil ausgelassen worden sind.

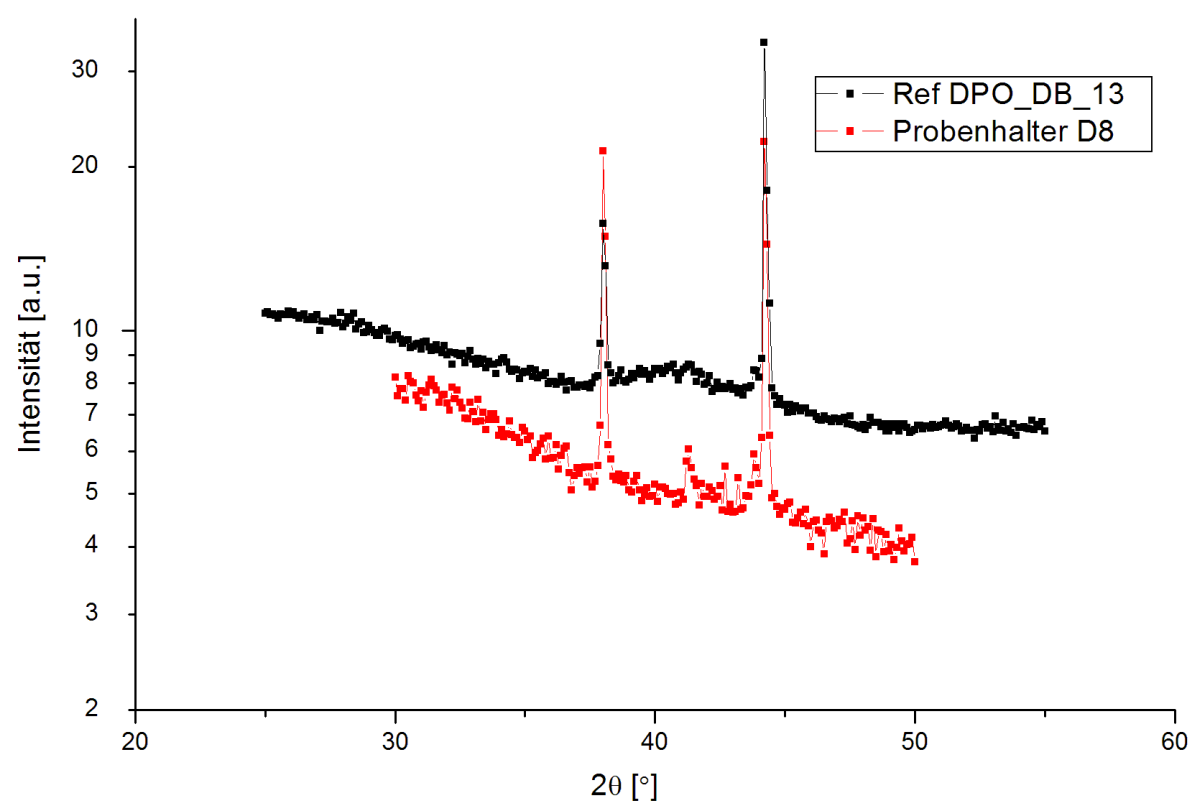

Abbildung A.1: Weitwinkel-Röntgenmessung der $10 \mathrm{~nm}$ dünnen PdCuSiSchicht auf Kapton ${ }^{\circledR}$. Ein amorpher Peak ist, wenn überhaupt, nur sehr schwach ausgeprägt. Es fehlen aber vollständig scharfe Reflexe, die der Probe zugeordnet werden können. Die verbleibenden scharfen Reflexe stammen von dem Probentisch des Röntgendiffraktometers D8. 


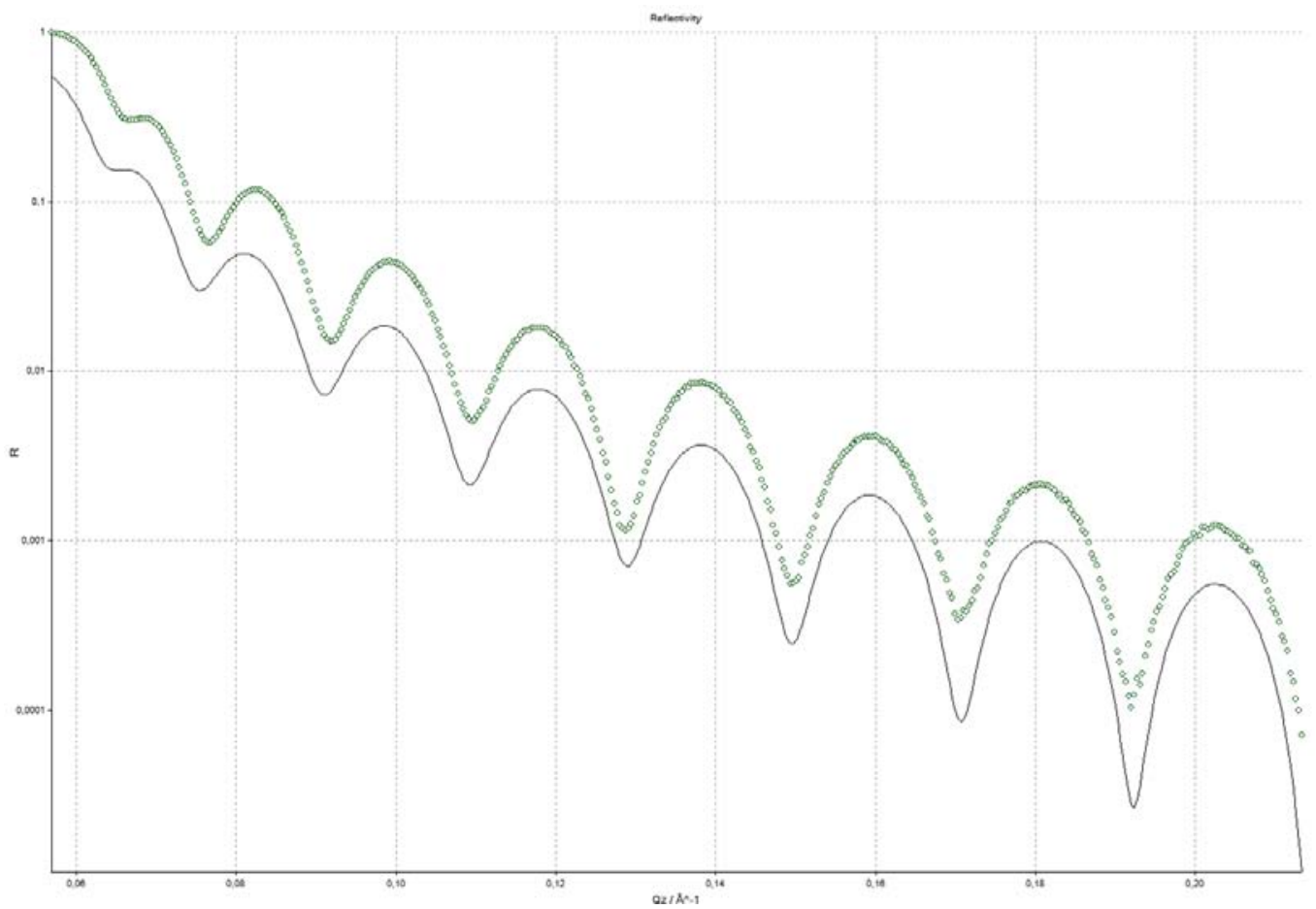

Abbildung A.2: Kleinwinkel-Röntgenreflektivität der Probe DPO_DB_08 (nominell $25 \mathrm{~nm} \mathrm{PdCuSi}$ auf Saphir) mit einer Parrat-Anppassung zu $27,5 \mathrm{~nm}$. Die Reflektivität ist gegen den Streuvektor $Q_{z}=4 \pi \frac{\sin (\Theta)}{\lambda}$ in $\AA^{-1}$ aufgetragen. Der Verlauf lässt sich gut anpassen, der Verlauf zeigt eine relativ glatte Schicht an.

\section{A.2 Weitere mechanische Messungen zu Schichten auf rauen Substraten}

An dieser Stelle werden die mechanischen Messungen von dünnen Filmen gezeigt, die auf sehr rauen Substraten hergestellt und vermessen wurden. Es wurde der Film DPO_DB_05a bei $730 \mathrm{~K}$ ausgelagert und nach erneuter „Leermessung“" ein Film mit nomineller Dicke 100 nm aufgebracht. Beim Aufdampfen konnte die Palladiumaufdampfrate nicht genau gehalten werden. Die Palladiumkonzentration in diesem Film liegt laut EDX Messung nur bei 69 at.\%. Die Messung der Dämpfung des Paddels mit und ohne diesen Film zeigt die Abbildung A.3.

Man erkennt eine von Null verschiedene Dämpfung durch den Film zu Beginn der Messung. Ohne ein vorheriges Ansteigen dieser Dämpfung verschwindet 


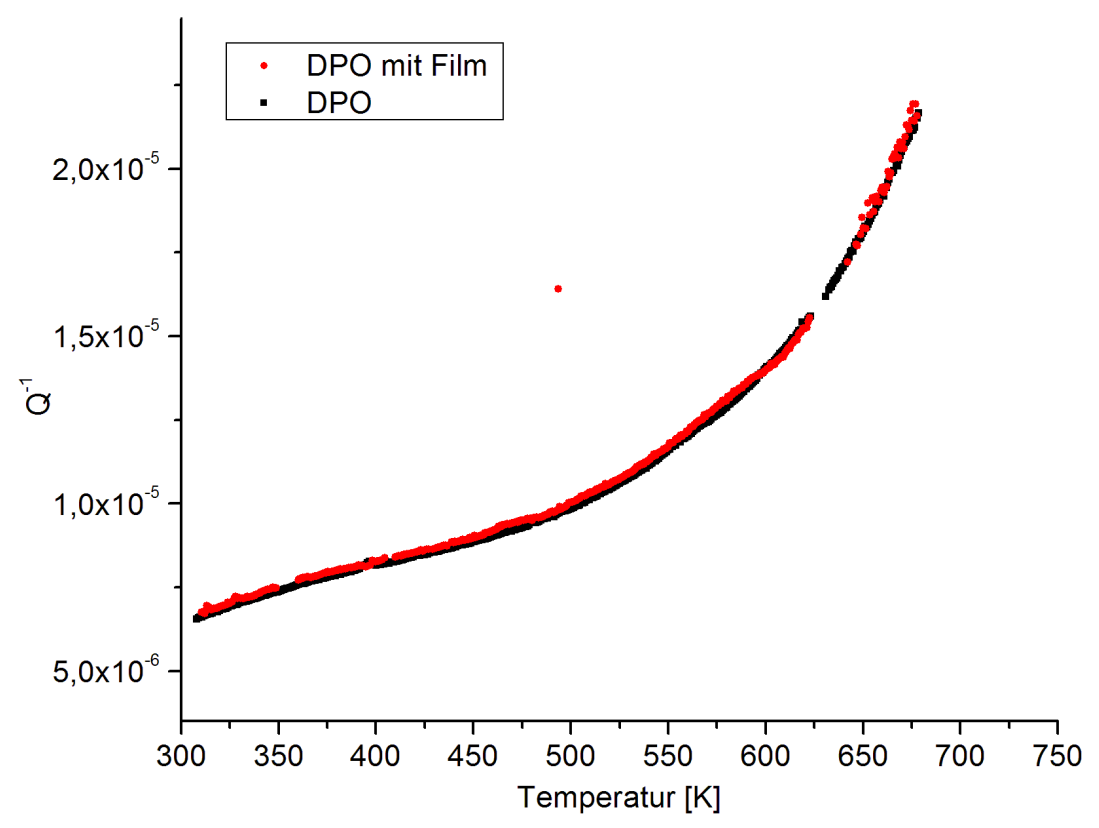

Abbildung A.3: Dämpfungsmessung eines PdCuSi-Filmes auf einem rauen Substrat (DPO_DB_05b). Die nominelle Schichtdicke beträgt $100 \mathrm{~nm}$.

dieser Unterschied bei etwa 600 K. Die Messung der Frequenzverschiebung liefert dazu einen Anstieg der Resonanzfrequenz bereits ab $350 \mathrm{~K}$, der bei $600 \mathrm{~K}$ abgeschlossen ist. Beide Messungen zusammen lassen sich so interpretieren, dass der Film, einerseits durch die nicht korrekte Zusammensetzung und andererseits durch das Vorliegen einer kristallinen Substratgrenzfläche, bereits früh zu kristallisieren beginnt. Die eigentliche Kristallisation scheint sich erst bei $600 \mathrm{~K}$ zu vollziehen, da dort die Dämpfung schlagartig zurückgeht. Die Tatsache, dass bis zu dieser Temperatur kein Anstieg in der Dämpfung zu beobachten ist, kann durch die Kristallisation bedingt sein. Andererseits ist dies aber auch mit dem Bild eines durch geometrischen „,confinement“ verhinderten $\beta$-Prozess vereinbar.

Um frühzeitige Kristallisation durch das bereits kristallisierte PdCuSi-Substrat zu vermeiden, wurde der Versuch unternommen, dies durch eine reine Palladiumschicht von $100 \mathrm{~nm}$ Dicke als Zwischenschicht zu verhindern. Mit der aufgebrachten Palladiumschicht konnte das Doppelpaddel wieder reproduzierbar vermessen und als ,leer" definiert werden. Auf diese Unterlage wurde ein nur $25 \mathrm{~nm}$ dicker PdCuSi-Film aufgedampft. Auch dieser Film weist im EDX zu wenig Palladium auf, Weitwinkel-Röntgenmessungen an der Referenzschicht und ein anfängliches Schermodul von $37 \mathrm{GPa}$ zeigen jedoch deutlich, dass der Film amorph hergestellt wurde. 


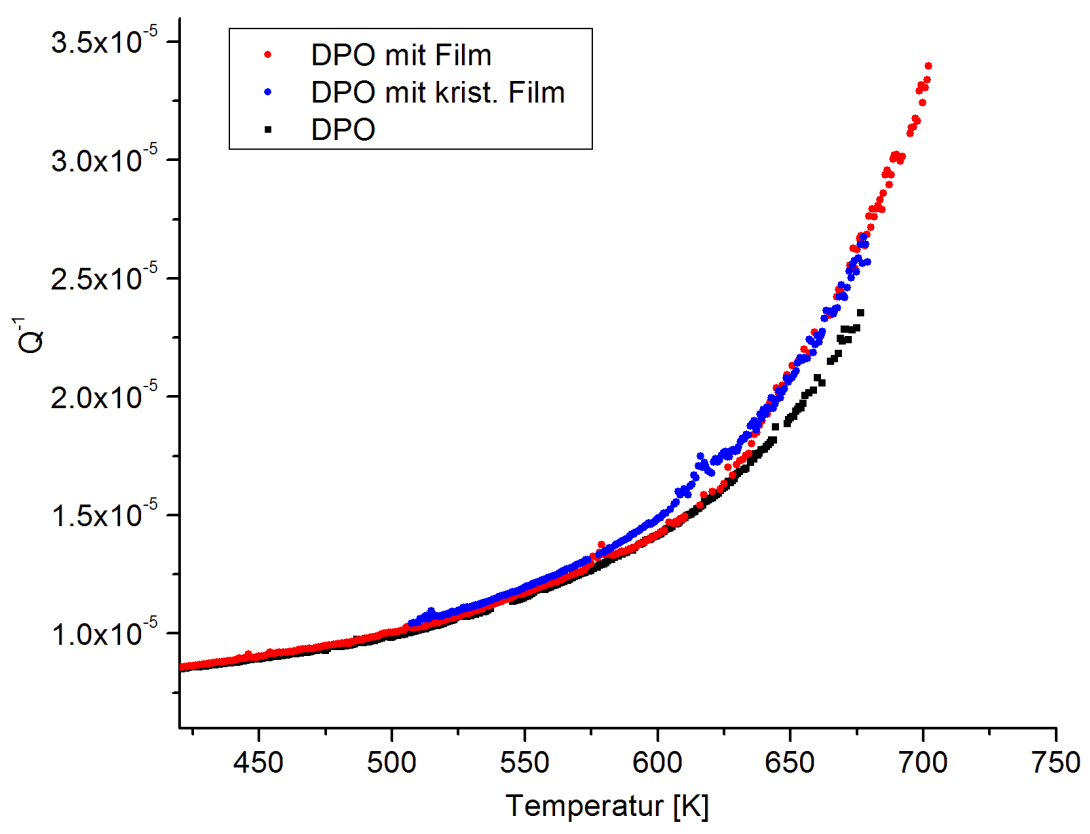

Abbildung A.4: Messung der Dämpfung durch einen $25 \mathrm{~nm}$ PdCuSi-Film (DPO_DB_05c) auf einer mehrlagigen Unterlage.

Die Vermessung dieses Film ist in Abbildung A.4 präsentiert. Bis etwa $630 \mathrm{~K}$ ist kein deutlicher Verlust durch den Film erkennbar, danach steigt die Dämpfung steil an. Ein zweiter Messdurchlauf mit dem nun als kristallin bezeichneten Film zeigt ansteigende Dämpfung bei noch geringerer Temperatur, zudem entspricht der zweite Messdurchlauf dem ersten weitgehend ab $640 \mathrm{~K}$. In der Frequenzmessung zeigt sich eine rasche Abnahme ebenfalls bei $630 \mathrm{~K}$. Die zweite Frequenzmessung zeigt gleiche Werte ab dieser Temperatur.

Bis $630 \mathrm{~K}$ wird also erneut keine Dämpfung durch den amorphen Film beobachtet. Der anschließende abrupte Ansteig in der Dämpfung kann nicht einer Relaxation des amorphen Film zugeordnet werden, da gleiche Dämpfungswerte auch im zweiten Messdurchlauf gemessen werden. Auch die schnelle, irreversible Änderung in der Resonanzfrequenz legt eher den Schluss nahe, dass die Sandwich-artige Struktur der Schichten z. B. aufgerissen ist. 


\section{Literaturverzeichnis}

[1] Askeland, Donald R.: Materialwissenschaften : Grundlagen, Lösungen, Übungen. Spektrum, Akad. Verl., 1996.

[2] ZARZYCKI, J. (Herausgeber): Materials Science and Technology - Volume 9: Glasses and Amorphous Materials. VHC Verlagsgesellschaft mbH, Weinheim, 1991.

[3] Elliot, S.R.: Physics of Amorphous Materials. Longmann Scientific \& Technical, 1990.

[4] Lunkenheimer, P., U. Schneider, R. Brand und A. Loidl: Glassy dynamics. Contemp. Phys., 41:15-36, 2000.

[5] Johari, G. P. und M. Goldstein: Viscous Liquids and the Glass Transition. II. Secondary Relaxations in Glasses of Rigid Molecules. J. Chem. Phys., 53:2372-2388, 1970.

[6] RÖsner, Peter Christian: Mechanische Relaxation in komplexen Fluiden. Doktorarbeit, I. Physikalisches Institut, Universität Göttingen, 2004.

[7] HACHENBERG, JÖRG: Sekundäre Relaxationen in amorphen Festkörpern Mechanische Spektroskopie an metallischen Gläsern und Copolymeren. Doktorarbeit, Universität Göttingen, 2006.

[8] Argon, A. S.: Plastic deformation in metallic glasses. Acta Metall., 27(1):47 - 58, 1979.

[9] TeICHLER, H.: Heterogeneous dynamics on the microsecond scale in simulated $N i_{0.5} Z r_{0.5}$ metallic melts far below the glass temperature. Phys. Rev. E, 71:031505, 2005.

[10] Zink, Mareike, K. Samwer, W. L. Johnson und S. G. Mayr: Plastic deformation of metallic glasses: Size of shear transformation 
zones from molecular dynamics simulations. Phys. Rev. B, 73:172203, 2006 .

[11] JÜlich, Forschungszentrum (Herausgeber): Dünne Schichten und Schichtsysteme, Vorlesungsmanuskripte des 17. IFF-Ferienkurses im Forschungszentrum Jülich. KFA, 1986.

[12] Rösner, P., K. Samwer, R. O. Pohl und S. Schneider: Use of a double-paddle oscillator for the study of metallic films at high temperatures. Rev. Sci. Instrum., 74:3395-3399, 2003.

[13] Debenedetti, Pablo G. und Frank H. Stillinger: Supercooled liquids and the glass transition. Nature, 410:259-267, 2001.

[14] Buckel, W. und R. Hilsch: Einfluß der Kondensation bei tiefen Temperaturen auf den elektrischen Widerstand und die Supraleitung für verschiedene Metalle. Z. Phys., 138:109-120, 1954.

[15] Inoue, A., T. Zhang und T. Masumoto: New amorphous alloys with significant supercooled liquid region and large reduced glass transition temperature. Mater. Sci. Eng. A, 134:1125-1128, 1991.

[16] Inoue, A. und N. Nishiyama: New Bulk Metallic Glasses for Applications as Magnetic-Sensing, Chemical, and Structural Materials. MRS Bulletin, 32:651, 2007.

[17] Mayr, S. G. und R. S. Averback: Surface smoothing of rough amorphous films by irradiation-induced viscous flow. Phys. Rev. Lett., 87:196106, 2001.

[18] Vauth, S. und S. G. MAYR: Ion bombardment induced smoothing of amorphous metallic surfaces: Experiments versus computer simulations. Phys. Rev. B, 77:155406, 2008.

[19] Cargill, G. S.: Dense Random Packing of Hard Spheres as a Structural Model for Noncrystalline Metallic Solids. J. Appl. Phys., 41:2248, 1970.

[20] Caprion, D., J. Matsui und H. R. Schober: Dynamic Heterogeneity of Relaxations in Glasses and Liquids. Phys. Rev. Lett., 85:4293, 2000.

[21] Richert, R. und K. SAmwer: Enhanced diffusivity in supercooled liquids. New J. Phys., 9:36, 2007. 
[22] Götze, W.: The essentials of the mode-coupling theory for glassy dynamics. Condens. Matter Phys., 1:873-904, 1998.

[23] Schober, H. R.: Soft phonons in glasses. Physica A, 201:14-24, 1993.

[24] Buchenau, U. und H. R. Schober: An atomic mechanism for the boson peak in metallic glasses. Phil. Mag., 88:3885, 2008.

[25] ANGell, C. A.: Structural instability and relaxation in liquid and glassy phases near the fragile liquid limit. J. Non-Cryst. Solids, 102:205, 1988.

[26] Kivelson, S. A. und G. TARJus: In search of a theory of supercooled liquids. Nature Materials, 7:831, 2008.

[27] Hecksher, T., N. B. Olsen A. I. Nielsen und J. C. Dyre: Little evidence for dynamic divergences in ultraviscous molecular liquids. Nat. Phys., 4:737, 2008.

[28] JÄCkle, J.: Models of the glass transition. Rep. Prog. Phys., 49:171, 1986.

[29] Ediger, M. D., C. A. Angell und S. R. NAGel: Supercooled Liquids and Glasses. J. Phys. Chem., 100:13200, 1996.

[30] Sokolov, A. P., A. Kisliuk, D. Quitmann, A. Kudlik und E. RÖSSLER: The dynamics of strong and fragile glass formers: vibrational and relaxation contributions. J. Non-Cryst. Solids, 172-174:138153, 1994.

[31] Hachenberg, J., D. Bedorf, K. Samwer, R. Richert, A. Kahl, M. D. Demetriou und W. L. Johnson: Merging of the $\alpha$ and $\beta$ relaxations and aging via the Johari-Goldstein modes in rapidly quenched metallic glasses. Appl. Phys. Lett., 92:131911, 2008.

[32] Снатнотн, S. M.: Structure and Dynamics of Al and Ni Based Melts Studied by Inelastic Neutron Scattering. Doktorarbeit, Technische Universität München, 2005.

[33] Lunkenheimer, P., R. Wehn, U. Schneider und A. Loidl: Glassy Aging Dynamics. Phys. Rev. Lett., 95:055702, 2005.

[34] Kahl, Annelen: Ultraschallspektroskopie an dem amorphen System $P d_{40} N i_{40} P_{20}$. Diplomarbeit, I. Physikalisches Institut, Universität Göttingen, 2006. 
[35] Sillescu, H.: Heterogeneity at the glass transition: a review. J. NonCryst. Solids, 243:81-108, 1999.

[36] Angell, C. A., K. L. Ngami, J. Kieffaer, T. Egami und G. U. Nienhaus (Herausgeber): MRS Symposium Proceedings Volume 455 Structure and Dynamics of Glasses and Glass Formers. Materials Research Society, 1997.

[37] LADADWA, I. und H. TEICHLER: Low-frequency transient dynamic clusters in simulated amorphous $N i_{0.5} Z r_{0.5}$ around the glass temperature. Phys. Rev. E, 78:041503, 2008.

[38] Adam, G. und J. H. GibBs: On the Temperature Dependence of Cooperative Relaxation Properties in Glass-Forming Liquids. J. Chem. Phys., 43:139, 1965.

[39] Johnson, W. L. und K. Samwer: A Universal Criterion for Plastic Yielding of Metallic Glasses with a $\left(T / T_{g}\right)^{2 / 3}$ Temperature Dependence. Phys. Rev. Lett., 95:195501, 2005.

[40] Ellison, C. J., S. D. Kim, D. B. Hall und J. M. Torkelson: Confinement and processing effects on glass transition temperature and physical aging in ultrathin polymer films: Novel fluorescence measurements. Eur. Phys. J. E, 8:155-166, 2002.

[41] Schönhals, A., H. Goering, Ch. Schick, B. Frick und R. Zorn: Glassy dynamics of polymers confined to nanoporous glasses revealed by relaxational and scattering experiments. Eur. Phys. J. E, 12:173-178, 2003.

[42] FakhraAi, Zahra und James A. Forrest: Probing Slow Dynamics in Supported Thin Polymer Films. Phys. Rev. Lett., 95(2):025701, 2005.

[43] Haramina, Tatjana: Mechanical spectroscopy of polymers with reduced dimensions and increasing cross-linking degree. Doktorarbeit, Universität Göttingen, 2006.

[44] Weeks, Eric R., J. C. Crocker, Andrew C. Levitt, Andrew Schofield und D. A. WeITZ: Three-Dimensional Direct Imaging of Structural Relaxation Near the Colloidal Glass Transition. Science, 287:627, 2000. 
[45] R. Schaller, G. Fantazzoni und G. Gremaud (Herausgeber): $M e$ chanical Spectroscopy $Q^{-1} 2001$ with Applications to Materials Science. TRANS TECH PUBLICATIONS LTD, 2001.

[46] http://en.wikipedia.org/wiki/Kelvin_material, Version vom 06.05.2009, Abbildung zur allgemeinen Verwendung freigegeben.

[47] Alvarez, F., A. Alegria und J. Colmenero: Interconnection between frequency-domain Havriliak-Negami and time-domain Kohlrausch-Williams-Watts relaxation functions. Phys. Rev. B, 47:125, 1993.

[48] Hachenberg, J., B. Steisel, U. Nergui, D. Bedorf, M. Buback und K. SAmwer: Effect of Chemical Confinement on the mechanical relaxation spectra of poly(ethene-co-methacrylic acid) copolymers. Int. J. Mater. Res. Z. Metallkd., 5:502 - 506, 2008.

[49] Menard, Kevin P.: Dynamic mechanical analysis: a practical introduction. CRC Press, 1999.

[50] Koeppe, Thomas: Temperaturabhängigkeit der elastischen Konstanten für das metallische Glas $P d_{40} N i_{40} P_{20}$. Diplomarbeit, I. Physikalisches Institut, Universität Göttingen, 2007.

[51] Turell, R., C. Elbaum und B. Chick: Ultrasonic Methods in Solid State Physics. Academic Press New York and London, 1969.

[52] Cappella, B. und G. Dietler: Force-distance curves by atomic force microscopy. Surf. Sci. Rep., 34:1-104, 1999.

[53] Rabe, U. und W. Arnold: Acoustic microscopy by atomic force microscopy. Appl. Phys. Lett., 64:1493-1495, 1994.

[54] Kopycinska-Müller, M., A. Caron, S. Hirsekorn, U. Rabe, H. Natter, R. Hempelmann, R. Birringer und W. Arnold: Quantitative Evaluation of Elastic Properties of Nano-Crystalline Nickel Using Atomic Force Acoustic Microscopy. Z. Phys. Chem., 222:471-498, 2008.

[55] VRee, Christian: Topographie, Struktur und Dynamik thermisch aufgedampfter Polymerfilme. Doktorarbeit, Universität Göttingen, 2009.

[56] Scharf, Thorsten: Depositionsmechanismen, Struktur und mechanische Eigenschaften laserdeponierter Poly(methyl methacrylat)-Filme. Doktorarbeit, Universität Göttingen, 2006. 
[57] Kleiman, R. N., G. K. Kaminsky, J. D. Reppy, R. Pindak und D. J. Bishop: Single-crystal silicon high-Q torsional oscillators. Rev. Sci. Instrum., 56(11):2088-2091, 1985.

[58] Spiel, Christoph L. und R. O. Pohl: Normal modes of a Si100 double-paddle oscillator. Rev. Sci. Instrum., 72:1482-1491, 2001.

[59] Ning, T. K. (Herausgeber): Properties of Silicon - Emis Datareviews Series, No. 4. Inspec, Iee, 1988.

[60] Liebermann, ERnst: Eine Näherung zur Voraussage des Zusatzvolumens binärer nichtassoziierter Mischungen. Monatshefte für Chemie, 108:505, 1977.

[61] Internetdatenbank http://www.webelements.com, weitere Quellenangaben dort.

[62] Tschuschke, Maria: Mechanische Spektroskopie an Poly(methyl methacrylat) und Biomolekuelen. Diplomarbeit, I. Physikalisches Institut, Universität Göttingen, 2006.

[63] Parrat, L. G.: Surface studies of solids by total reflection of x-rays. Phys. Rev., 95:359, 1954.

[64] Christian Braun, HMi Berlin: Parrat 32 or The Reflectivity Tool, 2002. Version 1.6.0, Programm frei verfügbar.

[65] Blanco, Jose Manuel, Fernando Flores und Rubén Pérez: STM-theory: Image potential, chemistry and surface relaxation. Progr. Surf. Sci., 81(10-12):403 - 443, 2006.

[66] Tseng, Ampere A. und Zhuang Li: Manipulations of Atoms and Molecules by Scanning Probe Microscopy. J. Nanosci. Nanotechnol., 7:2582-2595, August 2007.

[67] Seo, Yongho und Wonho Jhe: Atomic force microscopy and spectroscopy. Rep. Progr. Phys., 71:016101, 2008.

[68] Torre, J. Dalla, G. H. Gilmer, D. L. Windt, R. Kalyanaraman, F. H. Baumann, P. L. O’Sullivan, J. Sapjeta, T. Díaz DE LA Rubia und M. DJAFARI Rouhani: Microstructure of thin tantalum films sputtered onto inclined substrates: Experiments and atomistic simulations. J. Appl. Phys., 94(1):263-271, 2003.

[69] VREE, C.: persönliche Mitteilung, Februar 2009. 
[70] ChuA, L. F. und H. W. Kui: Correlation for the glass forming ability of $P d_{83.52-x} C u_{x} S i_{16.5}$ with crystalline-compound/solid-solution formation. J. of Appl. Phys., 84:5993, 1998.

[71] Holloway, Karen und Peter M. Fryer: Tantalum as a diffusion barrier between copper and silicon. Appl. Phys. Lett., 57:1736-1738, 1990.

[72] Holloway, Karen, Peter M. Fryer, Cyril Cabral, J. M. E. HARPER, P. J. BAILEY und K. H. Kelleher: Tantalum as a diffusion barrier between copper and silicon: Failure mechanism and effect of nitrogen additions. J. Appl. Phys., 71:5433-5444, 1992.

[73] BuschHorn, Stefan: Diffusionsmessungen an amorphen Multilagen mittels Neutronenreflektometrie. Diplomarbeit, I. Physikalisches Institut, Universität Göttingen, 2006.

[74] RöDER, J. und H.-U. KREBS: Frequency dependent smoothing of rough surfaces by laser deposition of $\mathrm{ZrO}_{2}$. Appl. Phys. Mater. Sci. Process., 90:609-613, 2008.

[75] HaAsen, Peter: Physikalische Metallkunde. Springer Verlag, 1994.

[76] MaYR, S. G.: Wachstum amorpher Schichten: Vergleich von Experiment und Simulation im Bereich Oberflächenrauhigkeit und mechanische Spannungen. Doktorarbeit, I. Physikalisches Institut, Universität Göttingen, 2000.

[77] Mayr, S. G., M. Moske und K. SAmwer: Early stages in amorphous $Z_{65} A l_{7.5} C u_{27.5}$ film growth on HOPG. Europhys. Lett., 44:465, 1998.

[78] White, B. E. und R. O. PoHL: Internal Friction of Subnanometer a-SiO ${ }_{2}$ Films. Phys. Rev. Lett., 75(24):4437-4439, Dec 1995.

[79] Liu, Xiao, S. F. Morse, J. F. Vignola, D. M. Photiadis, A. SarKissian, M. H. MARCus und B. H. Houston: On the modes and loss mechanisms of a high $Q$ mechanical oscillator. Appl. Phys. Lett., 78:1346-1348, 2001.

[80] Falbe, Jürgen und Manfred Regitz: Römp Lexikon Chemie. Georg Thieme-Verlag, 1996.

[81] Rösner, P., K. SAmwer und P. Lunkenheimer: Indications for an excess wing in metallic glasses from the mechanical loss modulus in $Z r_{65} A l_{7.5} C u_{27.5}$. Europhys. Lett., 68:226-232, 2004. 
[82] WeISS, MANFRED: Mechanische Eigenschaften und thermische Stabilität tief unterkühlter Metallschmelzen im Bereich der Glastemperatur. Doktorarbeit, Universität Augsburg, 1998.

[83] HachenBeRG, J. und K. SAMWER: Indications for a slow $\beta$-relaxation in a fragile metallic glass. J. Non-Cryst. Solids, 352:5110-5113, 2006.

[84] RicheRT, RANKO: Wide frequency range capacitive detection of loss in a metallic cantilever using resonance and relaxation modes. Rev. Sci. Instrum., 78:053901, 2007.

[85] SchwaBe, M.: persönliche Mitteilung über Creep-Recovery-Messungen an PdCuSi, August 2009.

[86] Vauth, Sebastian und S. G. Mayr: Atomic dynamics in molecular dynamics simulations of glassy CuTi thin films. Appl. Phys. Lett., 86:061913, 2005.

[87] PRITZ, T.: Verification of local Kramers-Kronig relations for complex modulus by means of fractional derivative model. J. Sound Vib., 228:1145-1165, 1999.

[88] Duan, Gang, M. L. Lind, M. D. Demetriou und W. L. Johnson: Strong configurational dependence of elastic properties for a binary model metallic glass. Appl. Phys. Lett., 89:151901, 2006.

[89] Harmon, J. S., M. D. Demetriou, W. L. Johnson und K. SamWER: Anelastic to Plastic Transition in Metallic Glass-Forming Liquids. Phys. Rev. Lett., 99:135502, 2007.

[90] Mayr, S. G.: Relaxation kinetics and mechanical stability of metallic glasses and supercooled melts. Phys. Rev. B, 79:060201, 2009.

[91] Koeppe, T., D. Bedorf, M. Schwabe und K. Samwer: Temperature dependence of the elastic constants for the metallic glass $P d_{40} N i_{40} P_{20}$. Poster MM 23.11, Frühjahrstagung der Deutschen Physikalischen Gesellschaft, 2008.

[92] SRIVAstava, S. K.: Relationship between elastic constants and thermal expansivity for ionic solids. Phys. B Condens. Matter., 387:396, 2007.

[93] Kittel, Charles: Einführung in die Festkörperphysik. R.Oldenburg Verlag München, 1968. ab Seite 59. 
[94] Eilers, G., H. Ulrichs, M. Münzenberg, A. Thomas, K. Thiel und M. SEIBT: Long-range order on the atomic scale induced at Co$\mathrm{FeB} / \mathrm{MgO}$ interfaces. J. Appl. Phys., 105:073701, 2009.

[95] Lang, M. J., M. Duarte-Dominguez, R. Birringer, R. Hempelmann, H. NATter und W. ARnold: Measurement of elastic and anelastic properties of nanocrystalline metals. Nanostruct. Mater., 12:811, 1999 .

[96] BöDdeker, B. und H. Teichler: Dynamics near free surfaces of molecular dynamics simulated $N i_{0.5} Z r_{0.5}$ metallic glass films. Phys. Rev. E, 59:1948, 1999.

[97] Weiss, Claus: Zum Glasübergang in metallischen Systemen. Diplomarbeit, Universität Augsburg, 1999.

[98] Eshelby, J. D.: The Determination of the Elastic Field of an Ellipsoidal Inclusion, and Related Problems. Proc. R. Soc. Lond. A, 241:376, 1957.

[99] Miracle, D. B., T. Egami, K. M. Flores und K. F. Kelton: Structural Aspects of Metallic Glasses. MRS Bulletin, 32:629, 2007.

[100] Champion, Y. und S. NowaK: Activation volume in fine grained metals from stress relaxation and nano-indentation. Mater. Sci. Forum, 584-586:399-404, 2008.

[101] Sharp, J. S. und J. A. Forrest: Free Surfaces Cause Reductions in the Glass Transition Temperature of Thin Polystyrene Films. Phys. Rev. Lett., 91(23):235701, Dec 2003.

[102] Volkert, C. A., A. Donohue und F. Spaepen: Effect of sample size on deformation in amorphous metals. J. Appl. Phys., 103:083539, 2008 .

[103] Volkert, C. A. und E. T. Lilleodden: Size effects in the deformation of sub-micron Au columns. Phil. Mag., 86:5567, 2006.

[104] Zhang, G. P., C. A. Volkert, R. Schwaiger, P. Wellner, E. ARzT und O. KRAFT: Length-scale-controlled fatigue mechanisms in thin copper films. Acta Mater., 54:3127-3139, 2006.

[105] Samwer, K.: persönliche Mitteilung, September 2009. 



\section{Danksagung:}

An dieser Stelle soll allen Menschen gedankt werden, die durch ihre Unterstützung diese Arbeit erst möglich gemacht haben.

Ich danke Herrn Professor Samwer, dass er mir die Möglichkeit der Promotion mit einem spannenden und herausfordernden Thema gegeben hat. Ich bin besonders dankbar für die unermüdliche Geduld und Zuversicht in das Gelingen der Experimente und die Freiheit dabei sehr selbständig zu arbeiten. Die vielen Diskussionen und Ratschläge in dieser Zeit haben mich dabei angeleitet, und ermutigt.

Herrn Professor Krebs danke ich für das Korreferat der Arbeit. Auch von Herrn Professor Krebs habe ich im Rahmen des Glasseminares und informellen Besprechungen viel über mechanische Spektroskopie und Polymerdynamik gelernt.

Frau Professorin Volkert als Teilprojektleiterin des Projektes B11 des Sonderforschungsbereiches 602 danke ich für ihr Interesse an meiner Arbeit und die fruchtbaren Diskussionen über Größeneffekte in kristallinen und amorphen Materialen.

Herrn Professor Richert möchte ich besonders danken für die experimentelle Zusammenarbeit während seiner Aufenthalte im I. Physikalischen Institut. Es war eine Freude mit einem Experten auf dem Gebiet der Glasphysik im Labor zu arbeiten. Ich habe dadurch neue experimentelle Techniken gelernt und viel über Glasdynamik.

Der Einstieg in die Technik des Doppelpaddel-Oszillators wurde mir durch das Arbeiten mit Andreas Meschede erleichtert. Ich danke ihm für die gute Zusammenarbeit, die durch Teamgeist geprägt war.

Allen Mitgliedern der Glasgruppe mit denen ich zusammen geforscht habe, danke ich für den tollen Zusammenhalt und die gegenseitige Unterstützung. Ich danke dabei Jörg Hachenberg, für die spannenden Diskussionsrunden, Stefan Buschhorn, Thomas Koeppe und Annelen Kahl für die gute Atmosphäre (Ich mach mal das Fenster auf...) und die immer kurzweiligen gemeinsamen Experimente und Gesprächsrunden. Ich danke Moritz Schwabe, Stefan Küchemann und Hannes Wagner für die angenehme Zusammenarbeit, Diskussionen zum Fortschritt meiner Arbeit und den guten Mannschaftsgeist in der Glasgruppe.

Ich danke allen Mitgliedern des I. Physikalischen Institutes für das gute Arbeitsklima und den freundlichen Umgang. Auch für die vielen kleinen Probleme, Fortschritte und Rückschritte gab es stets verlässliche Gesprächspartner, 
ich danke hierfür besonders Sebastian Vauth, Christian Vree, Tobias Edler und Iris Kock.

Dem Kollegen Yuansu Luo möchte ich für seine große Hilfsbereitschaft und die Unterstützung beim Beschichten der Paddel mit Tantal danken.

Sehr viel Freude hat auch die Zusammenarbeit mit den technischen Mitarbeitern gemacht, deren Unterstützung anspruchsvolle Experimenten erst ermöglichen. Ich danke an dieser Stelle Carsten Mahn, Uta Filippich, Katrin Gehrke, Ning An und Antje Krüger für ihre Mithilfe.

Die feinmechanische Werkstatt mit ihren motivierten und hilfsbereiten Mitarbeitern war eine große Hilfe beim Bau des Messeinsatzes und stets bereit für die kleinen „Notfälle“ beim Experimentieren. Herrn Eichenberg, Elmar Gatzemeier und Axel Bruchmann danke ich für diesen Einsatz.

Ich danke auch meinen Freunden, die außerhalb des Studiums den Kontakt gehalten haben.

Meinen lieben Eltern und Großeltern bin ich sehr dankbar für die uneingeschränkte Unterstützung, die ich während meines Studiums und der Promotion erhalten habe. Diese Unterstützung hat mich immer getragen und motiviert.

Meiner Freundin Anne-Katrin danke ich für die Liebe und Stärkung, die mich immer aufbaut und mein Leben täglich bereichert. 


\section{Publikationen:}

2007:

D. Bedorf and S. G. Mayr:

Grain boundary doping of $\mathrm{Cu}$ thin films with Bi: A route to create smooth and stable nanocrystalline surfaces.

Scripta Mater., 57:853, 2007

S. G. Mayr and D. Bedorf:

Stabilization of Cu nanostructures by grain boundary doping with Bi: Experiment versus molecular dynamics simulation.

Phys. Rev. B, 76:024111, 2007

2008:

Jörg Hachenberg, Dennis Bedorf, Konrad Samwer, Ranko Richert, Annelen Kahl, Marios D. Demetriou, and William L. Johnson:

Merging of the $\alpha$ and $\beta$ relaxations and aging via the Johari-Goldstein modes in rapidly quenched metallic glasses.

Appl. Phys. Lett., 92:131911, 2008

Jörg Hachenberg, Björn Steisel, Undrakh Nerguib, Dennis Bedorf, Michael Buback and Konrad Samwer:

Effect of Chemical Confinement on the mechanical relaxation spectra of poly(ethene-co-methacrylic acid) copolymers.

Int. J. Mater. Res. Z. Metallkd., 5:502, 2008.

\section{9:}

J. S. Kim, D. Bedorf, G. R. Stewart:

Effect of Disorder Induced by Heavy-Ion Irradiation on CeCoIn ${ }_{5}$ Superconductivity.

J. Low Temp. Phys., 157:29, 2009

A. Kahl, T. Koeppe, D. Bedorf, R. Richert, M. L. Lind, M. D. Demetriou, W. L. Johnson, W. Arnold, and K. Samwer:

Dynamical and quasi-static relaxation paths in $P d_{40} N i_{40} P_{20}$ glass.

Appl. Phys. Lett., 95:201903, 2009

Dennis Bedorf and Konrad Samwer:

Length Scale Effects on Relaxations in Metallic Glasses.

Accepted for publication in J. Non-Cryst. Solids 



\section{Lebenslauf:}

\section{Persönliche Angaben:}

Name:

Dennis Bedorf

Geboren am:

08.06.1980

Geburtsort:

Dortmund

Familienstand:

ledig

Staatsangehörigkeit:

Deutsch

\section{Schulausbildung:}

09.1986 bis 06.1990 :

Kirchhörder Grundschule in Dortmund

08.1990 bis 06.1999 :

Max-Planck-Gymnasium in Dortmund

01.06.1999:

Erwerb der Allgemeinen Hochschulreife

\section{Grundwehrdienst:}

01.11.1999 bis 31.08.2000: Sanitätskompanie in Hamm

\section{Hochschulausbildung:}

WiSe 2000 bis SoSe 2002: Grundstudium des Diplomstudiengangs Physik an der Universität Dortmund

09.2002 : Vordiplom in Physik

WiSe 2002 bis WiSe 2005: Diplomstudiengang Physik an der Georg-August-Universität Göttingen

04.2004 bis 09.2005: Anfertigung der Diplomarbeit unter der Anleitung von Prof. S. G. Mayr mit dem Titel:

„Oberflächenmorphologie und Mikrostruktur von dünnen Kupfer-Bismut-Zwangslegierungsschichten."

11.2005: Diplom, Note: ,sehr gut"

WiSe 2005:

seit 04.2006:

Wechsel zum Promotionstudiengang Physik

Anfertigung der vorliegenden Promotion als wissenschaftlicher Mitarbeiter am

I. Physikalischen Institut der Georg-August-Universität Göttingen, Betreuer der Promotion: Prof. Dr. Konrad Samwer 
